\title{
Deskription der Schwermetallgehalte in Knochen, Organen und Haaren von Fledermäusen (Chiroptera) \\ im Zeitraum 1987 bis 1999
}

\author{
Dissertation \\ zur Erlangung des Doktorgrades \\ der Mathematisch-Naturwissenschaftlichen Fakultäten \\ der Georg-August-Universität zu Göttingen
}

vorgelegt von

Rainer Hartmann

aus Hamburg

Göttingen 2000 
D 7

Referent: Prof. Dr. M. Schaefer Korreferent: PD Dr. H. Schutkowski

Tag der mündlichen Prüfung: 30.01.2001 


\section{Inhalt}

Seite

1. Einleitung 9

$1.1 \quad$ Fragestellung 9

1.2 Systematik und Nomenklatur 10

1.3 Habitatansprüche und Lebensweise der untersuchten Arten 12

$\begin{array}{ll}1.4 & \text { Haaranalytik zur Erfassung umweltbedingter Schwermetall- } \\ \text { expositionen } & 15\end{array}$

2. Material und Methodik 18

2.1 Herkunft des Tiermaterials 18

2.2 Aufarbeitung des Probenmaterials 19

2.2.1 Knochenproben 21

2.2.2 Organproben 22

2.2.3 Haarproben 22

2.2.4 Kotproben 24

2.3 Bestimmung des Schwermetallgehaltes 24

$2.4 \quad$ Statistische Methoden 25

3. Ergebnisse 26

$\begin{array}{ll}3.1 & \text { Übersicht über die verfügbaren Fledermaustotfunde aus } \\ \text { Niedersachsen } & 26\end{array}$

3.2 Zum Vorkommen der Mückenfledermaus 29

3.3 Bestimmung von Umrechnungsfaktoren zur Normierung anderer Untersuchungsergebnisse von Organproben auf $\begin{array}{ll}\text { die Trockensubstanz } & 32\end{array}$

3.4 Wasseraufnahmevermögen des Fledermaushaares 33

3.5 Qualitätssichernde Maßnahmen der Rückstandsanalytik 34

3.5.1 Kalibrierung 34

3.5.2 Abhängigkeit des Meßwertes von der Probeneinwaage 36

3.5.3 Variation der Knochenbleigehalte innerhalb eines Individuums 37

$\begin{array}{lll}3.6 & \text { Bleigehalte in Knochen } & 37\end{array}$

3.7 Schwermetallgehalte in Organen 43

$\begin{array}{lll}3.7 .1 & \text { Blei } & 44\end{array}$

$\begin{array}{lll}3.7 .2 & \text { Cadmium } & 46\end{array}$

$\begin{array}{lll}\text { 3.7.3 Chrom } & 47\end{array}$

3.8 Schwermetallgehalte in Haaren 51

3.8.1 Blei 51

3.8.2 Cadmium $\quad 55$

3.8.3 Chrom 56

3.8.4 Kupfer 57

3.8.5 Nickel 58

3.8.6 Zink 59 
Seite

3.9 Schwermetallgehalte im Kot 65

3.9.1 Aktuelle Schwermetallgehalte im Fledermauskot 65

$\begin{array}{lll}\text { 3.9.2 Veränderung der Schwermetallgehalte im Fledermauskot } & \\ \text { während der vergangenen zehn Jahre } & 66\end{array}$

3.10 Nachweis von Platin 68

4. Diskussion 69

4.1 Exposition, Bioverfügbarkeit und Toxikokinetik der Schwermetalle 69

4.2 Aussagefähigkeit und Interpretation der Schwermetallmessungen 72

4.2.1 Differenzierung zwischen endogener und exogener 72

4.2.2 Vergleich der Meßwerte mit Literaturangaben 74

4.2.2.1 Microchiroptera $\quad 75$

4.2.2.2 Megachiroptera $\quad 84$

4.2.3 Schwermetallgehalte im Fledermauskot 86

4.3 Ableitung von Referenzbereichen 91

4.3.1 Knochen 92

4.3.2 Organe 92

4.3.3 Haare 94

4.3.4 Kot 95

4.4 Auswirkungen der Substitution verbleiter Kraftstoffe auf die

$\begin{array}{ll}\text { 4.5 } & \text { Auswirkungen von Bodenbelastungen auf die } \\ \text { Schwermetallbelastung der Fledermäuse } & 97\end{array}$

$\begin{array}{lll}\text { 4.6 Auswirkungen wasserlöslicher Holzschutzmittel auf die } & \\ \text { Schwermetallbelastung von Fledermäusen } & 101\end{array}$

$\begin{array}{lll}\text { 4.7 Auswirkungen erhöhter Schwermetallbelastungen der Umwelt } & \\ \text { auf die Bestandsentwicklung der Fledermäuse } & 104\end{array}$

4.8 Platingruppenelemente in Umweltproben 106

$\begin{array}{ll}\text { 5. Zusammenfassung } & 107\end{array}$

6. Literatur 112 


\section{Abbildungsverzeichnis}

Seite

Abb. 1 Erläuterung der Box-Plot Darstellung

Verteilung der Anzahl an Fledermaustotfunden auf

die einzelnen Jahre

Abb. 3

Prozentuale Artenverteilung der Fledermaustotfunde

Abb. 10 bleigehalt beim Großen Mausohr

Abb. 11

Box-Plot-Diagramme der Knochenbleigehalte vom Großen

Mausohr und der Zwergfledermaus aus den Jahren 1993-1999

Abb. 12

Bleigehalte in Gewebeproben adulter Zwergfledermäuse

Abb. 16

Haarbleigehalte in Abhängigkeit von der Unterarmlänge (Alter) bei der Zwergfledermaus

Abb. 18

Quecksilbergehalte im Kot von Tardarida brasiliensis in

Abb. 20

Abhängigkeit der Produktion einer Kupferhütte bei Morenci (USA)

Abb. 21 Vergleich der errechneten Referenzwerte für Cadmium in Gewebeproben mit publizierten Literaturdaten

\section{Tabellenverzeichnis}

Tab. 1 Wissenschaftliche Bezeichnungen der behandelten

$$
\text { Fledermausarten }
$$

Tab. 4

$$
\text { Bevölkerung }
$$

Übersicht über die Anzahl der verfügbaren und untersuchten Individuen 
Seite

Tab. 10

Tab. 11

Tab. 12

Tab. 13

Tab. 14

Tab. 15

Tab. 16

Tab. 17

Tab. 18

Tab. 19

Tab. 20

Tab. 21

Tab. 22

Tab. 23

Tab. 24

Tab. 25

Tab. 26

Tab. 27

Tab. 28

Tab. 29

Tab. 30

Tab. 31

Tab. 32a

Tab. 32b

Tab. 32c

Tab. 33

Tab. 34

Tab. 35

Tab. 36

Tab. 37

Tab. 38

Ergebnisse der Doppelbestimmungen des Knochenbleigehaltes Medianwerte der Knochenbleigehalte von Zwergfledermäusen Statistische Kenngrößen der Knochenbleigehalte von Zwergfledermäusen

Zuordnung der Altersgruppe zur Unterarmlänge Medianwerte der Knochenbleigehalte von männlichen und weiblichen Zwergfledermäusen Statistische Kenngrößen der Bleigehalte in Gewebeproben von Zwergfledermäusen

Ergebnisse der U-Testprüfung zur Unterscheidung der Bleigehalte in Gewebeproben von Totfunden aus dem Harz und dem übrigen Niedersachsen

Statistische Kenngrößen der Chromgehalte in Gewebeproben von Zwergfledermäusen

Vergleich der statistischen Kenngrößen der Haarbleigehalte in Zwergfledermäusen

Medianwerte der Bleigehalte im Haar von männlichen und weiblichen Zwergfledermäusen

Medianwerte der Cadmiumgehalte im Haar bei

Zwergfledermäusen

der Cadmiumgehalte im Haar von männlichen und weiblichen Zwergfledermäusen

Medianwerte der Kupfergehalte im Haar bei Zwergfledermäusen

Medianwerte der Nickelgehalte im Haar von männlichen und weiblichen Zwergfledermäusen

Medianwerte der Zinkgehalte im Haar bei Zwergfledermäusen

Medianwerte der Zinkgehalte im Haar von männlichen und weiblichen Zwergfledermäusen

wermetallgehalte im Haar

der Zwergfledermäuse im Zeitraum 1987-1990

Statistische Kenndaten der Schwermetallgehalte im Haar der Zwergfledermäuse im Zeitraum 1991-1995

Statistische Kenndaten der Schwermetallgehalte im Haar der Zwergfledermäuse im Zeitraum 1996-1999

Statistische Kenngrößen der Fledermauskotuntersuchungen

Ergebnisse der Analyse von Kothaufen aus Wochenstuben

Ergebnisse der Glühverlustbestimmungen am Fledermauskot

Platingehalte in Kot, Knochen und Haaren von Fledermäusen

Schwermetallgehalte in Ganztieraufschlüssen des Großen

Mausohres aus dem südlichen Burgenland

Schwermetallgehalte in Ganztieraufschlüssen von 10 Fledermaus totfunden aus Darmstadt 
Seite

Tab. 39 Schwermetallgehalte in Ganztieraufschlüssen von Fledermaustotfunden aus Hessen (1981-1989)

Tab. 40

Schwermetallgehalte in Organen und Muskelgewebe von

10 Fledermäusen

Schwermetallgehalte von Knochen, Haaren und Krallen

Schwermetallgehalte in Organproben von Fledermäusen aus dem

Raum Darmstadt

Tab. 43

Cadmium- und (Methyl)-Quecksilbergehalte in Leber und Nieren

von Zwergfledermäusen aus Südschweden

Tab. 44

Elementgehalte im Haar vom Großen Mausohr aus Zentralpolen

Tab. 45

Bleigehalte im Ganztieraufschluß von Eptesicus fuscus und

Myotis lucifugus aus Montpelier Barn (USA)

Tab. 46

Schwermetallgehalte in Gewebeproben von Myotis austroriparius aus Florida (USA)

Blei- und Cadmiumgehalte von Tardarida brasiliensis im südlichen

Nordamerika

Tab. 48

Quecksilbergehalte in der Atemmuskulatur von verschiedenen

Fledermäusen aus dem südlichen Arizona (USA) und Mexico

Tab. 49

Schwankungsbreiten der Quecksilbergehalte in drei verschiedenen

Fledermausarten aus dem südlichen Japan

Tab. 50

Selengehalte in Organ- und Gewebeproben von drei Fledermäusen

Tab. 51

Bleigehalte in Gewebeproben von Pteropus poliocephalus aus

Brisbane mit Vergiftungssymptomen

Tab. 52

Vergiftungssymptomen

Tab. 53 Bleigehalte in Gewebeproben von Pteropus alecto aus Queensland (Australien)

Tab. 54

Blei- und Cadmiumgehalte in Kotproben aus einer Mausohrkolonie bei Neidhardshausen (Südthüringen)

Tab. 55

Blei- und Cadmiumgehalte in Kotproben aus Mausohrkolonien bei

Northeim und Hattorf/Harz

Tab. 56

Elementgehalte von zwei Kotproben aus einem Quartier des Großen Mausohres in der Ost-Alb (Baden-Württemberg)

Tab. 57

Schwermetallgehalte in Fledermauskot im Umfeld einer Batterieproduktion in der Region Jackson Country (Florida, USA)

Tab. 58 Elementgehalte von Kotproben von Myotis grisescens aus Kentucky (USA)

Tab. 59 Entwicklung der Blutbleigehalte und Haarbleigehalte beim Menschen während der Übergangsperiode von verbleiten zu unverbleiten Vergaserkraftstoffen

Tab. 60 Bleigehalte in Gewebeproben jagdbarer Wildarten aus dem Bereich des Harzes und anderen Revieren in Niedersachsen

Tab. 63 Vergleich der Zwergfledermäuse mit erhöhten Chrom- und Kupfergehalten im Haar 


\section{Anhang}

Anhang 1 Biometrische Daten Zwergfledermaus vs. Mückenfledermaus

Anhang 2 Meßwerte der Haarfeuchtebestimmungen

Anhang 3 Verfahrenskenndaten der Schwermetallanalytik

Anhang 4 Meßwerte der Organanalysen

Anhang 5 Detailangaben zu den untersuchten Tieren und Einzelmeßwerte

Anhang 6 Gegenüberstellung von Tieren mit hohen Haarbleigehalten

Anhang 7 Meßwerte der Kotanalysen 


\section{Einleitung}

\section{$1.1 \quad$ Fragestellung}

Schadstoffuntersuchungen an Fledermäusen, einer der am stärksten gefährdeten Säugetiergruppe in Deutschland, wurden in der Vergangenheit fast ausschließlich auf Organohalogenbelastungen (PCB, Organochlorpestizide wie DDT und dem Abbauprodukt DDE) durchgeführt (z.B. BRAUN 1986, DISSER und NAGEL 1987, NAGEL und Disser 1987, 1990, NAGel, Winter und Streit 1991, OHM 1991, NAGel 1998). Rückstandsanalysen auf Schwermetalle finden sich in der Literatur hingegen nur gelegentlich (z.B. Streit und NAGel 1993a, b, Gerell und Gerell 1993, ZYrNicki et al. 1996). Die wenigen publizierten Daten beziehen sich zudem auf kleine Stichprobengrößen oder behandeln außereuropäische Fledermausarten. Für die Bundesrepublik Deutschland und das europäische Ausland liegen bisher keine systematisch erhobenen Daten zum Schwermetallstatus der Fledermäuse vor. So ist bislang das Ausmaß der Bleibelastung in den achtziger Jahren des vergangenen Jahrhunderts bei Fledermäusen infolge der Verwendung von verbleitem Vergaserkraftstoff unbekannt. Auch ist der zu erwartende Rückgang der Bleibelastung durch die Einführung und Umsetzung des Benzinbleigesetzes unbekannt. Gleichfalls liegen fast keine Daten über die Schwermetallbelastung aus Holzschutzmitteln bei Fledermäusen vor.

Im Jahre 1979 wurden vom Verfasser mehr als 120 tote, überwiegend bereits stark mumifizierte Große Mausohren (Myotis myotis) in einer heute erloschenen Wochenstube bei Hattorf am Harz entdeckt. Die Tiere wurden eingesammelt, anhand der Zahnformel bestimmt und dem damaligen Landesamt für Naturschutz, Landschaftspflege und Vogelschutz, dem heutigen Niedersächsischen Landesamt für Ökologie (NLÖ) zur weiteren Rückstandsanalyse übersandt. In der Folgezeit wurden von zahlreichen, gleichfalls ehrenamtlich tätigen Personen aus Niedersachsen weitere Totfunde der Fachbehörde übersandt. Bis zum heutigen Tage fand sich im Haushalt der Behörde kein Finanztitel, aus dem die bereits vor über 20 Jahren vorgesehenen Rückstandsanalysen bezahlt werden konnten. Im Laufe der Jahre gingen Teile des Tiermaterials verloren oder wurden vernichtet. Die Restbestände lagern heute im Probenlager der Tiermedizinischen Hochschule Hannover.

Das über einen Zeitraum von rund 20 Jahren in Hannover archivierte Tiermaterial bot die seltene Gelegenheit der Rekonstruktion der Schwermetallbelastung von Fledermäusen über einen langen Zeitraum. In diese Zeit fielen u.a. die Einführung von bleifreiem Vergaserkraftstoff sowie die Installation und Aufrüstung von Rauchgasreinigungsanlagen in der Industrie.

Im Jahre 1998 erfolgte vom Verfasser eine erste Sichtung des noch vorhandenen Materials. Aufgrund des erhaltenen Bestandes von rund 400 Tieren wurde in Abstimmung mit dem Niedersächsischen Landesamt für Ökologie (NLÖ), Frau POTTDöRFER, beschlossen, dieses Material für Rückstandsanalysen auf Schwermetalle zu verwenden. Parallel zu den Untersuchungen am Tiermaterial wurden Kotproben aus 
rund 50 Wochenstuben und Männchenquartieren in Niedersachsen gesammelt und analysiert.

Mit diesen Untersuchungen soll u.a. der aktuelle Schwermetallstatus bei den stark im Bestand bedrohten Fledermäusen näher beschrieben werden. Anhand der gewonnenen Daten sollen ferner folgende Fragestellungen beantwortet werden:

- Führen regional erhöhte Schwermetallgehalte im Erdreich bei den sich fast ausschließlich von Fluginsekten ernährenden Fledermäusen zu einer nachweisbar erhöhten Schwermetallbelastung?

- Ist in Fledermäusen eine degressive Entwicklung der Bleibelastung seit der Einführung bleifreier Kraftstoffe feststellbar?

- Ergeben sich Hinweise auf eine Belastung der sogenannten Hausfledermäuse durch anorganische Holzbehandlungsmittel auf der Basis von Chrom- oder Kupfersalzen?

Darüber hinaus soll eine kritische Sichtung der zur Schwermetallbelastung von Fledermäusen vorliegenden Literatur vorgenommen werden. Auf der Grundlage der erhobenen Daten und der Literaturauswertung sollen Normalbereiche für Schwermetallkonzentrationen im Haar und im Kot der Fledermäuse sowie für Blei im Knochen abgeleitet werden, die es erlauben, künftig an Einzeltieruntersuchungen pathologische Schwermetallbelastungen erkennen zu können.

Gegen Abschluß der experimentellen Untersuchungen erschien die Publikation von HäUSSLER et al. (2000), in der erstmalig ein Bestimmungsschlüssel zur Differenzierung zweier sympatrisch vorkommender Zwergfledermausgruppen mit unterschiedlicher Ruffrequenz von $45 \mathrm{kHz}$ bzw. $55 \mathrm{kHz}$ enthalten war. Bereits seit mehreren Jahren wird diskutiert, ob es sich möglicherweise hierbei um zwei verschiedene Arten handelt, die bislang nicht unterschieden und stets als Zwergfledermaus (Pipistrellus pipistrellus) erfaßt wurden. Anhand des vorliegenden Tiermateriales erfolgte unter Anwendung des o.g. Bestimmungsschlüssels nochmals eine Nachbestimmung des Fundmateriales, primär mit dem Ziel, einen gesicherten Nachweis der neuen Art aus der Formengruppe „Zwergfledermäuse“ für Niedersachsen liefern zu können und eine Abschätzung der Häufigkeit des Auftretens dieser neuen, als Mückenfledermaus bezeichneten Art vorzunehmen.

\subsection{Systematik und Nomenklatur}

Die in der vorliegenden Arbeit verwendete Bezeichnung der einzelnen Fledermausarten orientiert sich an der Beschlußfassung des Fledermausreferates der EU, EUROBAT, vom Juli 1998 zur Übernahme der Nomenklatur gemäß der „3rd edition of the International Code of Zoological Nomenclature" (1985). Die verwendeten deutschsprachigen Bezeichnungen orientieren sich an der Publikation von Schober und GrimmBerger (1998). Tabelle 1 liefert einen Überblick über die behandelten Arten, deren systematische Stellung sowie deren aktuelle internationale und, soweit verfügbar, deutsche Bezeichnung. 
Tab. 1: Internationale wissenschaftliche Bezeichnungen der behandelten Fledermausarten gemäß den „rules of the 3rd edition of the International Code of Zoological Nomenclature" (nach KOOPMANN 1994) und deren gebräuchlicher deutschsprachiger Übersetzung

\section{Unterordnung Megachiroptera - Flederhunde}

Familie Pteropodidae - Flughunde

Pteropus poliocephalus TEMMINCK, 1825

Pteropus alecto TEMMINCK, 1837

Pteropus scapulatus PETERS, 1862 - Epouletten Flughund

Pteropus conspicillatus GOULD, 1850

Dobsonia moluccensis (QUOY u. GAIMARD, 1830)

\section{Unterordnung Microchiroptera - Fledermäuse}

Familie Vespertilionidae - Glattnasen-Fledermäuse

Barbastella barbastellus (SCHREBER, 1774) - Mopsfledermaus*

Eptesicus fuscus (BEAUVOIS, 1796) - Große Braune Fledermaus

Eptesicus nilssonii (KEYSERLING \& BLASIUS, 1839) - Nordfledermaus*

Eptesicus serotinus (SCHREBER, 1774) - Breitflügelfledermaus*

Myotis bechsteinii (KUHL, 1817) - Bechsteinfledermaus*

Myotis brandtii (EVERSMANN, 1845) - Große Bartfledermaus*

Myotis dasycneme (BOIE, 1825) - Teichfledermaus*

Myotis daubentonii (KUHL, 1817) - Wasserfledermaus*

Myotis emarginatus (GEOFFROY, 1806) - Wimperfledermaus

Myotis myotis (BORKHAUSEN, 1797) - Großes Mausohr*

Myotis mystacinus (KUHL, 1817) - Kleine Bartfledermaus*

Myotis nattereri (KUHL, 1817) - Fransenfledermaus*

Myotis lucifugus (LE CONTE, 1831) - Kleine Braune Fledermaus

Myotis vivesi MENEGAUX, 1901

Myotis grisescens HOWELL, 1909

Myotis austroriparius (RHOADS, 1897)

Nyctalus leisleri (KUHL, 1817) - Kleiner Abendsegler*

Nyctalus noctula (SCHREBER, 1774) - Großer Abendsegler*

Pipistrellus nathusii (KEYSERLING \& BLASIUS, 1839) - Rauhhautfledermaus*

Pipistrellus pipistrellus (SCHREBER, 1774) - Zwergfledermaus*

[Pipistrellus pygmaeus (LEACH, 1825) - Mückenfledermaus]

Plecotus auritus (LINNAEUS, 1758) - Braunes Langohr*

Plecotus austriacus (FISCHER, 1829) - Graues Langohr*

Vespertilio murinus LINNAEUS, 1758 - Zweifarbfledermaus*

Noctilio leporinus LINNAEUS, 1758 - Großes Hasenmaul

Familie Molossidae - Bulldogg-Fledermäuse

Tadarida brasiliensis (I. GEOFFROY, 1824) - Guano Fledermaus

*) in Niedersachsen vorkommende Arten (n. HECKENROTH und POTT 1988) 


\subsection{Habitatansprüche und Lebensweise der untersuchten Arten}

Bei den in Mitteleuropa vorkommenden Fledermäusen kann zwischen vergleichsweise ortstreuen Arten, mit Wanderstrecken bis zu etwa $50 \mathrm{~km}$ zwischen dem Sommer und dem Winterquartier, und wandernden Arten unterschieden werden. Letztere Arten legen Entfernungen zwischen dem Sommer- und dem Winterquartier von bis zu $2.000 \mathrm{~km}$ zurück, wie z.B. die Rauhhautfledermaus.

Je nach Präferenz bei der Wahl der Quartiere zur Jungenaufzucht, aber auch zur Überwinterung kann ferner zwischen den sogenannten Hausfledermäusen und den Fels- und Waldfledermäusen unterschieden werden. Die Hausfledermäuse, zu denen u.a. die Zwergfledermaus zählt, sind überwiegend ortstreue Arten, die ihren Hauptlebensraum in besiedelten Bereichen haben. Diese Arten sind daher auch von anthropogen freigesetzten Umweltschadstoffen in besonderem Maße betroffen, da der Schadstoffausstoß in besiedelten Bereichen, z.B. aus der Verbrennung fossiler Energieträger (Schwermetalle, polycyclische aromatische Kohlenwasserstoffe oder Benzol) oder der Anwendung von Holzschutzmittel im Dachstuhlbereich (Schwermetalle, Pestizide) deutlich höher ist als in ländlichen Regionen.

Eine Rückführung von nachgewiesenen Schadstoffbelastungen in Fledermäusen auf deren Herkunft ist nur bei ortstreuen Arten möglich, bei denen eine Erfassung des Lebensumfeldes möglich ist. Arten wie die Zwergfledermaus oder das Große Mausohr bieten hierfür sinnvolle Ansatzpunkte.

Im Gegensatz zu zahlreichen tropischen, sich von Früchten und Nektar ernährenden Flughunden (CANNON 1999) sind alle mitteleuropäischen Fledermausarten carnivor und stehen aufgrund ihrer nächtlichen und verborgenen Lebensweise am Ende der Nahrungskette. Die Zahl der natürlichen Feinde ist gering und beschränkt sich auf gelegentliches Jagdglück von Greifvögeln oder Hauskatzen, die in Sommerquartiere eindringen bzw. an den Ausfluglöchern vor allem in das Quartier zurückkehrende Fledermäuse erhaschen.

Die Nahrung der Fledermäuse schwankt je nach präferiertem Lebensraum und Jahreszeit und besteht überwiegend aus Fluginsekten. Beschrieben ist aber auch das Auflecken von Beutetieren von Blattoberflächen, wobei die Fledermäuse dann oft kurzzeitig im Rüttelflug zur Orientierung vor der Beute stehen, oder das gelegentliche Aufnehmen z.B. von Käfern von der Bodenoberfläche, dies ist besonders für das Große Mausohr bekannt. Die präferierte Nahrung der im nachfolgenden intensiver untersuchten Fledermäuse, der Zwergfledermaus und dem Großen Mausohr, entspricht deren Körperbau. Während sich die kleine, kaum $4 \mathrm{~cm}$ große Zwergfledermaus zu $50 \%$ von Dipteren (primär Chironomiden) und nur zu etwa $10 \%$ von Coleopteren ernährt (SWIFT et al. 1985, EICHSTÄDT und BASSUS 1995), beträgt der Anteil an Coleopteren beim fast amselgroßen Großen Mausohr im Jahresmittel über $90 \%$. Hiervon machen Carabiden mit 70-80 \% den Hauptnahrungsanteil aus. Dipteren dienen dem Großen Mausohr dagegen nicht als Nahrungsquelle (BAUEROVA 1978). Der tägliche Nahrungsbedarf beträgt etwa $1 / 4$ bis $1 / 3$ des Körpergewichtes 
(SCHOBER und GRIMMBERGER 1998). Für die Zwergfledermaus läßt sich somit die täglich benötigte Nahrungsmenge auf 2-3 g abschätzen, für das Große Mausohr auf etwa $10 \mathrm{~g}$. Tabelle 2 gibt eine Übersicht über die Habitatansprüche und Lebensweise der in Niedersachsen vorkommenden Fledermausarten.

Tab. 2: Habitatansprüche, Wanderungen und Ernährung der in Niedersachsen vorkommenden Fledermäuse (n. SCHOBER u. GRIMMBERGER 1998 u.a.)

\begin{tabular}{|c|c|c|c|}
\hline Spezies & Lebensraum & Wanderung & Nahrung \\
\hline $\begin{array}{l}\text { Mopsfledermaus (Bar- } \\
\text { bastella barbastellus) }\end{array}$ & $\begin{array}{l}\text { Waldfm.; waldreiche } \\
\text { Gebirgs- und Vorge- } \\
\text { birgsregionen, auch in } \\
\text { Siedlungen }\end{array}$ & $\begin{array}{l}\text { ortstreu, um } 15 \text { km; } \\
\max .290 \mathrm{~km}\end{array}$ & $\begin{array}{l}\text { überw. Lepidoptera der } \\
\text { Fam. Pyralidae und } \\
\text { Arctiidae }\end{array}$ \\
\hline $\begin{array}{l}\text { Nordfledermaus (Epte- } \\
\text { sicus nilssonii) }\end{array}$ & $\begin{array}{l}\text { Waldfm.; aufgelockerte } \\
\text { Busch- und Waldgeb. } \\
\text { vorw. Nadelwald, auch } \\
\text { in Ortschaften }\end{array}$ & ortstreu, max. $115 \mathrm{~km}$ & $\begin{array}{l}\text { Diptera, Lepidoptera, } \\
\text { Neuroptera, Hemiptera }\end{array}$ \\
\hline $\begin{array}{l}\text { Breitflügelfledermaus } \\
\text { (Eptesicus serotinus) }\end{array}$ & $\begin{array}{l}\text { Hausfm.; am Rande } \\
\text { menschl. Siedlungen, } \\
\text { auch Großstädte, ferner } \\
\text { in Parks, Gärten, Wie- } \\
\text { sen }\end{array}$ & ortstreu, max. $330 \mathrm{~km}$ & $\begin{array}{l}\text { Coleoptera, Lepidopte- } \\
\text { ra, Diptera; nimmt auch } \\
\text { Na. vom Boden auf }\end{array}$ \\
\hline $\begin{array}{l}\text { Gr. Bartfledermaus } \\
\text { (Myotis brandtii) }\end{array}$ & $\begin{array}{l}\text { Waldfm.; Gewässer- } \\
\text { ränder in Waldnähe u. } \\
\text { Wälder }\end{array}$ & $\begin{array}{l}\text { Wanderer, max. bis } \\
700 \mathrm{~km}\end{array}$ & $\begin{array}{l}\text { Lepidoptera, Tipulidae, } \\
\text { Diptera, gelegentlich } \\
\text { Brachycera u. Arachni- } \\
\text { da=> Na. aufnahme } \\
\text { auch von Pflanzenober- } \\
\text { fläche }\end{array}$ \\
\hline $\begin{array}{l}\text { Bechsteinfledermaus } \\
\text { (Myotis bechsteinii) }\end{array}$ & $\begin{array}{l}\text { Waldfm.; vorw. feuchte } \\
\text { Mischwälder aber auch } \\
\text { Kiefernwald, Parks, } \\
\text { Gärten }\end{array}$ & ortstreu, max. 39 km & $\begin{array}{l}\text { Lepidoptera, Diptera, } \\
\text { Arachnida, Opilionides, } \\
\text { Dermaptera, aber auch } \\
\text { Raupen und flugunfähi- } \\
\text { ge Käfer. }\end{array}$ \\
\hline $\begin{array}{l}\text { Teichfledermaus (Myo- } \\
\text { tis dasycneme) }\end{array}$ & $\begin{array}{l}\text { Hausfm.; Wochenstu- } \\
\text { ben überw. in Siedlun- } \\
\text { gen, ansonsten in } \\
\text { feuchten Gebieten mit } \\
\text { Wiesen und Wäldern, } \\
\text { überw. im Tiefland }\end{array}$ & $\begin{array}{l}\text { Wanderer über } 100 \mathrm{~km} \text {, } \\
\text { max. } 330 \mathrm{~km} \text {, zieht im } \\
\text { Herbst in südl. Richtung }\end{array}$ & $\begin{array}{l}\text { vorw. Chironomidae, } \\
\text { Trichoptera, Lepidopte- } \\
\text { ra und Coleoptera. } \\
\text { Na.aufnahme erfolgt } \\
\text { auch von der Wasser- } \\
\text { oberfläche }\end{array}$ \\
\hline $\begin{array}{l}\text { Wasserfledermaus } \\
\text { (Myotis daubentonii) }\end{array}$ & $\begin{array}{l}\text { Waldfm; Flachland, of- } \\
\text { fene Wälder, Parks }\end{array}$ & $\begin{array}{l}\text { Wanderer, sucht Som- } \\
\text { merquartiere im Um- } \\
\text { kreis von rund } 100 \mathrm{~km} \\
\text { um das Winterquartier }\end{array}$ & $\begin{array}{l}\text { Diptera, Trichoptera, } \\
\text { Neuroptera, } \\
\text { Lepidoptera }\end{array}$ \\
\hline $\begin{array}{l}\text { Gr. Mausohr } \\
\text { (Myotis myotis) }\end{array}$ & $\begin{array}{l}\text { Hausfm. (in Norddt.); } \\
\text { an menschl. Siedl. ge- } \\
\text { bunden, ansonsten lich- } \\
\text { te baumbest. Land- } \\
\text { schaften, Parks }\end{array}$ & $\begin{array}{l}\text { ortstreu, meist um } 50 \\
\mathrm{~km}, \text { max. } 390 \mathrm{~km}\end{array}$ & $\begin{array}{l}\text { Coleoptera (vorw. Ca- } \\
\text { rabidae), Opilionides, } \\
\text { Raupen. Es ist auch der } \\
\text { Fang einer Spitzmaus } \\
\text { beschrieben. }\end{array}$ \\
\hline
\end{tabular}


noch Tabelle 2:

KI. Bartfledermaus
(Myotis mystacinus)

Fransenfledermaus (Myotis nattereri)

\author{
Gr. Abendsegler \\ (Nyctalus noctula)
}

Kl. Abendsegler (Nyctalus leisleri)

Rauhhautfledermaus

(Pipistrellus nathusii)

Graues Langohr

(Plecotus austriacus)

Braunes Langohr

(Plecotus auritus)

Zwergfledermaus (Pipistrellus pipistrellus)

bfledermaus

(Vespertilio murinus) eher Haus- als

Waldfm.; Parks, Gär-

ten, Dörfer

vw. Waldfm.; Wälder und Parks in Gew.nähe, 60 km, max.185 km gelegentl. Dörfer

Waldfm.; geleg. auch größere Parks

Waldfm., Lebensraum ähnl. Gr. Abendsegler.

Waldfm.; feuchte Laubwälder aber auch trockene Kieferforste, überw. im Flachland, selten in Siedlungen

Hausfm.; in Dörfern und Städten, auch in Parks und Wälder

Waldfm.; lockere Laubund Nadelwälder, auch Parks und Gärten, in Siedlungen jedoch nicht an menschl. Siedlungsraum gebunden

Hausfm.; bevorzugt Kulturlandschaften mit warmen Tallagen und menschl. Siedlungen, meidet größere Waldgebiete

Felsfm.; im Flachland Hausfm. u.a an Hochhäusern anzutreffen $240 \mathrm{~km}$ Herbst nach Südwe-

Wanderer, zieht im Herbst nach Südwesten, max. $1.052 \mathrm{~km}$

Wanderer, zieht im $1.905 \mathrm{~km}$ $\max .770$ km*

ortstreu, max. $66 \mathrm{~km}$

ortstreu, um 20 km; $\max .62 \mathrm{~km}$

Wander, zieht im $1.440 \mathrm{~km}$ eher ortstreu, max. bis

Lepidoptera auch Arachnida u. Raupen (absammeln der Vegetation)

Arachnida, Diptera, geleg. Lepidoptera und Coleoptera. Sucht wahrsch. primär Pflanzen ab.

Diptera, Trichoptera, Coleoptera, Lepidoptera sten, z.T. mit Schwalben gemeinsam beobachtet, max. $2.347 \mathrm{~km}$ Herbst nach SW, max.

ortstreu, um 10-20 km Herbst nach SW, max.
Lepidoptera, Diptera,

Trichoptera

Chironomidae, seltener

Neuroptera u. Hemiptera (nur Fluginsekten, die an Gewässernähe gebunden sind)

Diptera, Coleoptera,

Trichoptera,

Lepidoptera (nur

Fluginsekten)

Lepidoptera, Diptera,

Dermaptera, aber auch

Arachnida und Raupen.

Sammelt Beute im freien Luftraum und durch Ablesen.

vorw. Lepidoptera (Noctuidae), gelegentlich auch Diptera, Coleoptera

vorw. Diptera, aber auch Lepidoptera, Coleoptera, Trichoptera, Neuroptera, offenbar nur Fluginsekten

\footnotetext{
*) Von 15.000 beringten Tieren in Mecklenburg-Vorpommern und Brandenburg wurden sechs Tiere in einer Entfernung von
} mehr als $100 \mathrm{~km}$ wieder aufgefunden. 


\subsection{Haaranalytik zur Erfassung umweltbedingter Schwermetallexpositionen}

Die Untersuchung des Elementgehaltes von Haaren, speziell von Humanhaar, zur Erfassung von Umwelteinflüssen auf den Probanden oder zur Erkennung von Mangelerkrankungen hat in den vergangenen Jahren erheblich an Bedeutung gewonnen. Während erste Haaruntersuchungen, vorrangig zur Erfassung morphologischer Unterschiede, bereits vor über 100 Jahren unter kriminologischen Gesichtspunkten zur Klärung möglicher Täter-Opferbeziehungen durchgeführt wurden, stieg ab Anfang der 80er Jahre das Interesse an Haaruntersuchungen zur Deskription besonderer Stoffwechsellagen, pathologischer und homöostatischer Vorgänge im Organismus.

Der Vorteil der Haaranalytik zur Erfassung von umweltbedingten Schadstoffbelastungen im Vergleich zu anderen Untersuchungsmethoden am Patienten oder am lebenden Tier liegt vor allem in der einfachen Gewinnung von Probenmaterial. Bei Exhumierungen oder Totfunden sind die Haare neben den Knochen zudem das einzige verwertbare Spurenmaterial. Urin- oder Blutuntersuchungen liefen jeweils nur ein Abbild der augenblicklichen Belastungssituation. Mit Haaruntersuchungen können auch mehrere Monate zurückliegende Intoxikationen erkannt und zeitlich zugeordnet werden. Knochenanalysen gestatten im Vergleich zu Haaranalysen einen Rückblick auf noch weiter zurückliegende Intoxikationen, eine zeitliche Zuordnung ist jedoch anhand von Knochenuntersuchungen nicht mehr möglich. Auch ist der Aufwand der Probengewinnung am lebenden Objekt nur mit einem operativen Eingriff möglich. Ähnliches gilt auch für die Rückstandsanalyse an Zahnmaterial. Bei humantoxikologischen Fragestellungen bietet sich hier allerdings die Möglichkeit der Untersuchung von Milchzähnen an. Derartige Studien liefern selbstverständlich nur Rückschlüsse auf die aktuelle Belastungssituation bei Kindern.

Klassische Anwendungsfälle der Haaruntersuchung sind die Erfassung von Thallium- und Arsenintoxikationen, in neuerer Zeit aber auch die Erfassung eines unerlaubten Konsums von Anabolika und Rauschmittel. Neben der vergleichsweise einfachen Probengewinnung bietet die Haaranalytik gegenüber der Untersuchung von Körperflüssigkeiten oder Gewebeproben den großen Vorteil der zeitlichen Zuordnung der Intoxikation bzw. des Konsumes. Das menschliche Haar wächst im Mittel $10 \mathrm{~mm}$ im Monat. Bei der Untersuchung einzelner Haarsegmente kann somit ein einmaliger oder kurzzeitiger Konsum bzw. eine einmalige Intoxikation von Dauerbelastungen unterschieden werden. Eine ausführliche Darstellung der Bedeutung, aber auch der Möglichkeiten der Mißdeutung von Haaranalysen zur Klärung von Intoxikationen oder anderen kriminalistischen Fragestellungen liefern KIJEWSKI und LANGE (1977) und KIJEWSKI $(1993,1997)$.

Der Schwermetallgehalt der Haare unterliegt vielfältigen Einflüssen. Hierzu zählen u.a. Alter, Geschlecht, Ernährungsgewohnheiten, exogene Umwelteinflüsse, aber auch die Lage des Probenabschnittes entlang der Haarachse und die anatomische Lokation (KATZ und CHATT 1988). Zusätzlich kommen beim Humanhaar Faktoren wie Raucher/Nichtraucher, Haarwaschverhalten, berufsbedingte Expositionen und angewandte Haarfärbetechniken hinzu. So wurde im Rahmen des Umweltmonitorings 
von KRAUSE et al. (1997) für Aluminium z.B. ein signifikanter Unterschied bei Frauen mit und ohne Dauerwellenfrisur nachgewiesen. Frauen mit einer Dauerwelle hatten mit 3,4 $\mu \mathrm{g} / \mathrm{g}$ einen signifikant höheren Aluminiumgehalt im Haar als Frauen ohne Dauerwelle $(2,31 \mu \mathrm{g} / \mathrm{g})$. Die Stichprobengröße betrug hierbei 1660 untersuchte Frauen.

Die Auswirkungen der oralen Aufnahme von Schwermetallen auf den Schwermetallstatus der Haare wurde auf der Grundlage von Fütterungsversuchen von ANKE und RISCH (1979) untersucht.

Ähnliche Untersuchungen wurden an Ratten und Rindern von STERNER (1972) vorgenommen. STERNER kommt zu dem Ergebnis, daß der Bleigehalt im Knochen der über Beimischung von Bleinitrat im Tränkewasser exponierten Versuchstiere (Wistarratten) in hohem Grade mit dem Bleigehalt im Haar der Versuchstiere positiv korreliert ist $(r=0,941)$.

RosmaNITH et al. (1976) untersuchten die Beziehungen zwischen den Blei- und Zinkimmissionen von Industrieanlagen in Nordrhein-Westfalen und den Konzentrationen von Blei, Cadmium und Zink in Blut, Urin und in den Haaren von Kindern. Die Studie beruht auf einer Untersuchung von rund 400 Kindern. In der Studie kamen die Autoren zu dem Ergebnis, daß mit steigender Schwermetallimmissionsrate die Bleigehalte im Blut und im Haar der untersuchten Kinder stiegen. Zink- und Cadmiumimmissionen führten zum Anstieg dieser Schwermetalle im Urin. Kinder aus der Stadtmitte wiesen zudem im Vergleich zu Kindern aus dem Stadtrandbereich derselben Industriestadt signifikant höhere Gehalte von Blei, Cadmium und Zink im Haar auf. Gleichfalls erhöht war bei der Gruppe aus dem Innenstadtbereich der Blutbleispiegel.

Haaruntersuchungen wurden und werden auch im Zusammenhang mit der Untersuchung der Schadstoffexpositionen am Arbeitsplatz durchgeführt. Untersuchungen an Werktätigen können dabei vorhandene Mängel z.B. in der Schadstoffabsaugung aufdecken. Untersuchungen an nicht mehr im Erwerbsleben stehenden Personen können mögliche frühere berufsbedingte Expositionen zur Rechtfertigung möglicher Rentenansprüche klären. GRUND et al. (1980) führten im Rahmen von arbeitshygienischen Untersuchungen Mangananalysen an Haaren von Elektroschweißern von vier Großwerften durch. Untersucht wurden 460 Schweißer und 450 Nichtschweißer. Der Mangangehalt im Kopfhaar der Schweißer lag rund fünffach über denen der Nichtschweißer.

KIJEWSKI und LOWITZ (1982) wendeten im Rahmen eines berufsgenossenschaftlichen Anerkennungsverfahren bei der Untersuchung ehemaliger Bleiarbeiter aus dem Nordharz die Haaruntersuchung zur Vorauswahl von Patienten mit Verdacht auf bleiinduzierter Nephropathie an. Bei erhöhten Bleigehalten im Haar im Vergleich zu einem Vergleichskollektiv aus Sektionsfällen wurde an den Patienten zusätzlich eine Beckenkammbiopsie durchgeführt und an dem entnommenen Knochengewebe anschließend eine Bleibestimmung vorgenommen. Von 13 untersuchten Patienten wiesen 12 sowohl erhöhte Haarblei- als auch Knochenbleigehalte auf. 
SPÄTH und SONNEBORN (1987) kommen in ihren Untersuchungen zur dem Ergebnis, daß die Haaranalyse dann sinnvoll einsetzbar ist, wenn sie zur Erkennung oder zur Abgrenzung von Kollektiven mit erhöhtem Risiko, z.B. im Umfeld von Hütten oder nach Störfällen, in Verbindung mit weiteren Untersuchungen eingesetzt wird.

Grundsätzlich besteht bei Haaruntersuchungen zur Abschätzung der Schwermetallinkorporation immer die Gefahr der Verfälschung von Meßergebnissen durch exogene Verunreinigungen. In Einzelfällen kann jedoch auch das vorherige Waschen der Haare keine vollständige Entfernung exogen anhaftender oder aufgebrachter Verunreinigungen bewirken. Auch können durch das Waschen Metallionen weiter in das Haarinnere hineintransportiert werden, statt diese zu entfernen (KIJEWSKI und LANGE 1977).

Die gegenwärtig umfangreichste Studie zur Schwermetallverteilung im Humanhaar wurde im Rahmen des bundesdeutschen Umwelt-Surveys im Auftrage des Bundesministeriums für Umwelt, Naturschutz und Reaktorsicherheit in den Jahren 1990 bis 1992 durchgeführt (KRAUSE et al. 1997). Untersucht wurden Kinder (6-14 jährig) und Erwachsene (25-69 jährig) in den neuen und alten Bundesländern auf insgesamt 18 Spurenelemente sowie auf Cotinin und Nikotin. Der Gesamtstichprobenumfang betrug 3905 Erwachsene und 718 Kinder. Die im Rahmen des Human-Biomonitoring 1990/1992 ermittelten durchschnittlichen Substanzgehalte im Haar der deutschen Bevölkerung sind in Tabelle 3 zusammengefaßt dargestellt.

Tab. 3: Durchschnittliche Spurenelementgehalte im Haar der deutschen Bevölkerung (neue und alte Bundesländer) nach den Erhebungen des HumanBiomonitorings 1990/1992 (KRAUSE et al. 1997)

\begin{tabular}{|c|c|c|c|c|}
\hline \multirow[b]{2}{*}{ Element } & \multicolumn{2}{|c|}{ Kinder (6-14 jährig) } & \multicolumn{2}{|c|}{ Erwachsene (25-69 jährig) } \\
\hline & $\begin{array}{c}\text { arithm. Mittel } \\
{[\mu \mathrm{g} / \mathrm{g} \text { TS }]}\end{array}$ & $\begin{array}{c}\text { Median } \\
{[\mu g / g \text { TS }]}\end{array}$ & $\begin{array}{c}\text { arithm. Mittel } \\
{[\mu \mathrm{g} / \mathrm{g} \text { TS }]}\end{array}$ & $\begin{array}{c}\text { Median } \\
{[\mu \mathrm{g} / \mathrm{g} \text { TS] }}\end{array}$ \\
\hline Aluminium & 9,20 & 6,9 & 4,73 & 3,1 \\
\hline Barium & 0,54 & 0,4 & 0,98 & 0,5 \\
\hline Blei & 1,61 & 1,0 & 2,26 & 0,9 \\
\hline Cadmium & 0,096 & 0,05 & 0,084 & 0,04 \\
\hline Calcium & 394 & 250 & 856 & 440 \\
\hline Chrom, ges. & 0,106 & 0,09 & 0,144 & 0,12 \\
\hline Kupfer & 24,8 & 12 & 19,1 & 12 \\
\hline Magnesium & 25,8 & 15 & 52,5 & 26 \\
\hline Phosphor & 119,2 & 114 & 145,7 & 140 \\
\hline Strontium & 0,99 & 0,5 & 2,22 & 1,0 \\
\hline Zink & 155 & 160 & 166 & 170 \\
\hline
\end{tabular}




\section{Material und Methodik}

\subsection{Herkunft des Tiermateriales}

Alle in Niedersachsen sowie der überwiegende Teil der in Deutschland vorkommenden Fledermausarten stehen als Tiergruppe mit besonders hohem Gefährdungsgrad auf der Roten Liste der gefährdeten Tiere und Pflanzen (NOWAK et al. 1994, BoYE et al. 1999). Darüber hinaus gelten zahlreiche der in Deutschland vorkommenden Fledermausarten europaweit als "Tierarten von gemeinschaftlichem Interesse“ im Sinne der Richtlinie 92/93 EWG des Rates der Europäischen Gemeinschaften zur Erhaltung der natürlichen Lebensräume sowie der wildlebenden Pflanzen und Tiere (FFHRichtlinie). Für ökotoxikologische Studien verbietet es sich daher, lebende Tiere zu fangen und für Untersuchungszwecke zu töten und zu sezieren. Das dieser Untersuchung zugrundeliegende Tiermaterial stammt daher von Totfunden oder von erkrankten Fledermäusen, die bei der Pflege gestorben sind. Dieses Tiermaterial wird seit über 20 Jahren von der Niedersächsischen Fachbehörde für Naturschutz, dem heutigen Niedersächsischen Landesamt für Ökologie, über ehrenamtlich tätige Fledermausbeauftragte gesammelt und an der Tiermedizinischen Hochschule Hannover durch Tiefgefrieren konserviert.

Im Rahmen dieser Arbeit wurde das an der Tiermedizinischen Hochschule Hannover gelagerte Material erstmals vollständig gesichtet und erfaßt. Hierbei wurden folgende Angaben in einer ACCESS-Datenbank erfaßt:

- Tierart

- Geschlecht

- Alter

- Funddatum

- Fundort und Fundumstände

- verwendetes Verpackungsmaterial

- Erhaltungszustand.

Die im Probenlager der Tiermedizinischen Hochschule Hannover erhobenen Daten wurden zu einem späteren Zeitpunkt durch im Labor erhobene Daten zum Gewicht, zur Unterarmlänge sowie durch die Meßergebnisse der Schwermetallanalysen in der Datenbank ergänzt.

Sämtliche Tiere erhielten zur eindeutigen Identifikation eine Registriernummer. Diese wurde sowohl auf der Umverpackung als auch auf dem Probenbegleitschein notiert. Sämtliche individuumbezogenen Daten stehen somit für mögliche spätere Untersuchungen am selben Tiermaterial zur Verfügung. Die im MICROSOFT ${ }^{\circledR}$-ACCESS-Format erzeugte Datenbank befindet sich in Kopie u.a. beim Niedersächsischen Landesamt für Ökologie sowie an der Tiermedizinischen Hochschule Hannover. 
Zur Gewinnung absicherbarer Daten ist ein ausreichendes Probenkollektiv von wesentlicher Bedeutung. Unter Berücksichtigung des vorliegenden Tiermateriales wurde daher entschieden, die Rückstandsuntersuchungen ausschließlich an Zwergfledermäusen (Pipistrellus pipistrellus) durchzuführen. Für Knochenuntersuchungen wurden ergänzend noch Proben vom Großen Mausohr (Myotis myotis) mit herangezogen, da hier zusätzliche Skelettfunde aus einer erloschenen Wochenstube bei Hattorf, Landkreis Osterode am Harz, vorlagen. Die zur Verfügung stehende Probenanzahl wurde eingeschränkt durch einen z.T. schlechten Erhaltungszustand der Tiere oder bei Jungtieren durch eine fehlende ausreichende Behaarung. Insgesamt wurden 111 Knochenproben von Zwergfledermäusen, 30 Knochenproben vom GroBen Mausohr sowie 95 Haarproben von Zwergfledermäusen untersucht (Tab. 4). Von insgesamt 22 Zwergfledermäusen wurden zudem Organproben entnommen und auf ausgewählte Schwermetalle untersucht (Tab. 5). Andere Fledermausarten wurden nur stichprobenartig auf die Schwermetallbelastung analysiert:

$\begin{array}{ll}\text { Myotis mystacinus - Bartfledermaus } & 4 \text { Ex. } \\ \text { Myotis bechsteinii - Bechsteinfledermaus } & 1 \text { Ex. } \\ \text { Myotis nattereri - Fransenfledermaus } & 1 \text { Ex. } \\ \text { Pipistrellus nathusii - Rauhhautfledermaus } & 1 \text { Ex. } \\ \text { Plecotus auritus - Braunes Langohr } & 1 \text { Ex. }\end{array}$

Aufgrund der geringen Datendichte zu diesen Arten finden die Meßergebnisse im nachfolgenden keine Berücksichtigung. Sie sind lediglich in der Zusammenstellung der Rohdaten im Anhang 5 der Vollständigkeit halber mit aufgeführt.

\subsection{Aufarbeitung des Probenmateriales}

Die Auswahl der Tiere für die Rückstandsanalysen erfolgte primär nach dem Erhaltungszustand. Da es sich bei dem bearbeiteten Tiermaterial überwiegend um Totfunde handelte, waren viele der Tiere bereits von Maden zerfressen oder, bei Funden aus Wochenstuben, mumifiziert, so daß Organproben nur gelegentlich in guter Qualität entnommen werden konnten. Gute Qualität hatten hingegen Organe aus konservierten Tieren von vergeblichen Rehabilitationsversuchen, z.B. von verletzten Exemplaren.

Die Gewinnung der Analysenproben erfolgte unter Verwendung von Instrumenten aus chirurgischem Stahl. Vorversuche mit menschlichem Haarmaterial zeigten, daß kein signifikanter Abrieb von Chrom und Nickel von dem verwendeten Skalpell- und Scherenmaterial erfolgt.

Sämtliche Laborarbeiten einschließlich der Analysen erfolgten in den Laborräumen der Gesellschaft für angewandte Biologie und Geologie mbH, Göttingen. 
Tab. 4: Übersicht über die Anzahl der verfügbaren und untersuchten Individuen, verteilt auf die einzelnen Jahresklassen (Betrachtungszeiträume)

a) Knochen:

Harz + Umgebung:

\begin{tabular}{r|c|c|c}
\hline \hline Zeitraum & $\begin{array}{c}\text { verfügbare Ex. } \\
\text { P. pipistrellus }\end{array}$ & $\begin{array}{c}\text { untersuchte Ex. } \\
\text { P. pipistrellus }\end{array}$ & $\begin{array}{c}\text { untersuchte Ex. } \\
\text { M. myotis }\end{array}$ \\
\hline$\leq 1990$ & 9 & 8 & 10 \\
\hline $1991-1995$ & 72 & 10 & \\
\hline$\geq 1996$ & 115 & 49 & \\
\hline Summe & 196 & 67 & 10 \\
\hline \hline
\end{tabular}

übriges Niedersachsen:

\begin{tabular}{rccc}
\hline \hline Zeitraum & $\begin{array}{c}\text { verfügbare Ex. } \\
\text { P. pipistrellus }\end{array}$ & $\begin{array}{c}\text { untersuchte Ex. } \\
\text { P. pipistrellus }\end{array}$ & $\begin{array}{c}\text { untersuchte Ex. } \\
\text { M. myotis }\end{array}$ \\
\hline$\leq 1990$ & 20 & 14 & \\
$1991-1995$ & 16 & 16 & 20 \\
$\geq 1996$ & 24 & 17 & 20 \\
\hline Summe & 60 & 47 & \\
\hline \hline
\end{tabular}

b) Haare:

Harz + Umgebung:

\begin{tabular}{rcc}
\hline \hline Zeitraum & $\begin{array}{c}\text { verfügbare Ex. } \\
\text { P. pipistrellus }\end{array}$ & $\begin{array}{c}\text { untersuchte Ex. } \\
\text { P. pipistrellus }\end{array}$ \\
\hline$\leq 1990$ & 9 & 6 \\
$1991-1995$ & 72 & 10 \\
$\geq 1996$ & 115 & 37 \\
\hline Summe & 196 & 53 \\
\hline \hline
\end{tabular}

übriges Niedersachsen:

\begin{tabular}{rcc}
\hline \hline Zeitraum & $\begin{array}{c}\text { verfügbare Ex. } \\
\text { P. pipistrellus }\end{array}$ & $\begin{array}{c}\text { untersuchte Ex. } \\
\text { P. pipistrellus }\end{array}$ \\
\hline$\leq 1990$ & 20 & 14 \\
$1991-1995$ & 16 & 11 \\
$\geq 1996$ & 24 & 17 \\
\hline Summe & 60 & 42 \\
\hline \hline
\end{tabular}


Tab. 5: Anzahl der untersuchten Organproben von Zwergfledermäusen (P. pipistrellus)

\begin{tabular}{lccc}
\hline \hline \multicolumn{1}{c}{ Fundort } & Lunge & Leber & Nieren \\
\hline Harz + Umgebung & 10 & 10 & 12 \\
übriges Niedersachsen & 7 & 10 & 10 \\
\hline Summe & 17 & 20 & 22 \\
\hline \hline
\end{tabular}

\subsubsection{Knochenproben}

Die Entnahme von Knochenmaterial erfolgte durch Präparationen der Unterarmknochen. Der herausgetrennte Unterarmknochen wurde zunächst von den Resten der anhaftenden Flughaut und dem Muskelgewebe befreit. Sodann erfolgte das Abtrennen der distalen Gelenke sowie der Ulna. Unter Verwendung von warmem Wasser wurde manuell das Knochenmark herausgespült und das Periost entfernt. Nach dieser Reinigung wurde dreimal mit Aqua. demin nachgespült. Abschließend erfolgte ein dreimaliges Spülen mit Aceton zur Rückstandsanalyse (РROMOCHEM, Art.-Nr. 0018).

Die von Geweberesten befreiten und gewaschenen Knochen wurden auf PE-Folie gelegt und mit einer weiteren Lage PE-Folie überdeckt, so daß beim Zerteilen keine Knochensplitter verlorengehen konnten. Anschließend erfolgte mit einem scharfen Skalpell durch die PE-Folie hindurch das Zerteilen der Knochen in 3-5 mm lange Stücke, Unterarmknochen von adulten Tieren mit mehr als $1 \mathrm{~mm}$ Durchmesser wurden zudem einmal in der Längsachse zerteilt.

Die Knochenstücke wurden mit einer Pinzette in 1,5 ml-Eppendorf-Reaktionsgefäße (Safe-lock) gebracht und für 6 Stunden im Trockenschrank bei $80^{\circ} \mathrm{C}$ getrocknet. Zur Vermeidung möglicher Staubeinträge, z.B. beim Öffnen des Trockenschrankes, wurde das Probenrack mitsamt den Aufschlußgefäßen zuvor in einen beiderseits umgefalteten hitzebeständigen Kunststoffolienschlauch geschoben.

Zur Entfettung wurde 0,5 ml Pentan (Merck SupraSolv, Art.-Nr. 1.16739) in die Reaktionsgefäße gegeben. Die Gefäße wurden sodann für 20 Minuten automatisch geschüttelt. Anschließend erfolgte das Absaugen des Pentans. Zur vollständigen Entfernung des Knochenfettes wurde noch dreimal mit Pentan nachgewaschen. Die so entfetteten Knochen wurden für 2 Stunden erneut in den Folienschlauch geschoben und im Trockenschrank bei $80^{\circ} \mathrm{C}$ getrocknet. Anschließend erfolgte das Auswiegen auf $0,01 \mathrm{mg}$ genau.

Der eigentliche Aufschluß erfolgte mit 0,5 ml 65 \%iger Salpetersäure (MERCK Suprapur, Art.-Nr. 1.00441) in den Reaktionsgefäßen. Nach Säurezugabe blieben die Gefäße zunächst bis zum Abschluß der Gasblasenbildung für etwa 2 Stunden bei 
Raumtemperatur stehen. Anschließend erfolgte ein langsames Temperieren der druckdicht verschlossenen Gefäße für 12 Stunden auf $60^{\circ} \mathrm{C}$. Nach dieser Zeit wurde auf $80^{\circ} \mathrm{C}$ aufgeheizt. Die Endtemperatur wurde für 4 Stunden gehalten.

Die so vollständig aufgeschlossenen Knochenproben wurden nach dem Abkühlen mit 0,5 ml Reinstwasser (MERCK, Art.-Nr. 1.16754) verdünnt und bis zur Analyse im Kühlschrank gelagert.

\subsubsection{Organproben}

Die herauspräparierten Organproben wurden kurz in Reinstwasser abgespült und mit Zellstoff abgetupft. Anschließend wurden die Organe in zuvor auf 0,01 mg ausgewogene 1,5 ml-Eppendorf-Reaktionsgefäße (Safe-lock) gegeben und nach dem Tiefgefrieren gefriergetrocknet. Zur Gefriertrocknung wurde eine Anlage vom Typ Alpha 1-4 der Firma Christ verwendet. Die gefriergetrockneten Proben wurden unmittelbar nach dem Herausnehmen aus der Gefriertrocknungsanlage auf 0,01 mg genau zurückgewogen. Der Probenaufschluß erfolgte wieder in der zuvor beschriebenen Weise mit $0,5 \mathrm{ml}$ Salpetersäure Suprapur. Die vollständig aufgeschlossenen Gewebeproben wurden nach dem Abkühlen mit 0,5 ml Reinstwasser (MERCK, Art.-Nr. 1.16754) verdünnt und bis zur Analyse im Kühlschrank gelagert.

\subsubsection{Haarproben}

Die Präparation erfolgte auf einer Kunststoffolie, die nach jedem präpariertem Tier gewechselt wurde. Die Arbeitsfläche wurde unmittelbar vor Beginn der Präparationen feucht gereinigt. Die Arbeiten erfolgten in einem staubfreien Laborraum bei abgeschalteter Umluft, um mögliche Haar- und Staubverwirbelungen zu vermeiden.

Die für die Untersuchung verwendeten Haare wurden mittels Präparationsschere etwa 0,5-1 mm über der Hautoberfläche abgetrennt. Primär erfolgte dabei die Probengewinnung im gesamten dorsalen Bereich. Gab es nur wenig dorsale Haare, z.B. bei juvenilen Tieren, wurden auch ventrale Haare präpariert. Vor dem eigentlichen Abtrennen der Haare erfolgte je nach Verschmutzungsgrad des Felles eine mechanische Reinigung durch vorsichtiges Abbürsten mittels Pinsel oder der Entfernung von Wollfasern oder Milben mittels Pinzette.

Die insgesamt erhaltene Probenmenge betrug um $30 \mathrm{mg}$ je Tier, bei Jungtieren um $10 \mathrm{mg}$. Aufgrund der geringen Haarlängen von 3-5 mm erfolgte keine zusätzliche Abtrennung der Haarspitzen oder eine Auswahl der Haarmittelbereiche für die Analytik, wie dies z.B. bei der Untersuchung von Humanhaar üblich ist. 
Im Anschluß an den Präparationsschritt erfolgte das Waschen der Haare. Dies wurde in $40 \mathrm{ml}$-Glasröhrchen, die mit 0,1 molarer Salpetersäure vorgereinigt wurden, bei Raumtemperatur gemäß IAEA-Methodenvorschrift (IAEA 1978) wie folgt durchgeführt:

- $10 \mathrm{ml}$ Aceton (Promochem, Art.-Nr. 0018) wurden zum Probenmaterial hinzugegeben und für 10 min maschinell geschüttelt. Nach Ablauf dieser Zeit wurden die Haare mit einer Pinzette herausgenommen und in ein anderes Glasröhrchen gegeben.

- $10 \mathrm{ml}$ Reinstwasser (MeRCK, Art.-Nr. 1.16754) wurden hinzugegeben und für 10 min maschinell geschüttelt. Nach Ablauf dieser Zeit wurden die Haare mit einer Pinzette herausgenommen. Das Glasröhrchen wurde ausgespült und erneut mit dem Probenmaterial befüllt. Dieser Waschvorgang wurde noch zweimal wiederholt.

- $10 \mathrm{ml}$ Aceton wurden zum gewaschenen Probenmaterial hinzugegeben und für 10 min maschinell geschüttelt. Nach Ablauf dieser Zeit wurden die Haare mit einer Pinzette herausgenommen und in ein 1,5 ml-Eppendorf-Reaktionsgefäß gegeben.

Der Waschschritt diente der Abtrennung von exogenen Verunreinigungen auf der Haaroberfläche. Bedingt durch die Verhältnisse am Fundort der Tierleiche war eine unterschiedlich starke Verunreinigung der Körperoberfläche erfolgt. Dies war besonders stark bei Jungtieren oder Weibchen aus Wochenstuben der Fall. Herabfallender Kot und Urin anderer Tiere führte zum Verkleben des Felles. Totfunde von der Erdoberfläche konnten zudem mit anhaftendem Bodenmaterial verunreinigt sein. Auch im Fell von frischtoten Tieren können mineralische Ablagerungen von Staub eine erhebliche exogene Verunreinigung bewirken. Über das Für und Wider der Haarreinigung, aber auch über die sinnvollste Reinigungstechnik existiert umfangreiche Literatur. Eine kritische Betrachtung findet sich in der Diskussion (Kap. 4.2.1).

Die für diese Untersuchung ausgewählte Reinigungsmethode gemäß IAEAMethodenvorschrift hat sich in den letzten Jahren als Standardverfahren herausgebildet. So wurde dieses Reinigungsverfahren auch bei der bundesdeutschen HumanBiomonitoringstudie des Bundesministeriums für Umwelt, Naturschutz und Reaktorsicherheit im Rahmen des Umwelt-Survey 1990/1992 bei einer Gesamtprobandenanzahl von 3.900 Personen angewandt (KRAUSE et al. 1997).

Die gewaschenen Haare wurden in den 1,5 ml-Eppendorf-Reaktionsgefäßen für $12 \mathrm{~h}$ im Trockenschrank bei $80^{\circ} \mathrm{C}$ getrocknet. Zur Vermeidung von Staubeinträgen, z.B. beim Öffnen des Trockenschrankes, waren die offenen Reaktionsgefäße zuvor samt Probenrack in einen Folienschlauch geschoben worden. Nach dem Trocknen wurden die offenen Reaktionsgefäße in eine staubdichte Kunststoffbox mit $50 \%$ relativer Luftfeuchtigkeit gestellt und für mindestens 3 Tage äquilibriert. Die Kontrolle der 
Luftfeuchte in der Box erfolgte über ein Haarhygrometer. Nach erfolgter Angleichung der Haarfeuchte wurde das Probenmaterial in zuvor auf 0,01 mg ausgewogene 1,5 ml-Safe-lock-Eppendorf-Reaktionsgefäße umgefüllt, die Reaktionsgefäße verschlossen und auf der Analysenwaage zurückgewogen. Die Äquilibrierung bei $50 \%$ Luftfeuchte diente zur Einstellung eines einheitlichen Feuchtegehaltes der zu analysierenden Haarproben.

Der eigentliche Probenaufschluß erfolgte mit $0,5 \mathrm{ml} 65 \%$ iger Salpetersäure (MERCK Suprapur, Art.-Nr. 1.00441) in den Eppendorf-Reaktionsgefäßen nach der bei SPERLING und BAHR (1979) beschriebenen Methode. Nach Säurezugabe blieben die Gefäße zunächst bis zum Abschluß der Gasblasenbildung für etwa 2 Stunden bei Raumtemperatur stehen. Anschließend erfolgte ein langsames Temperieren der druckdicht in einem Aluminiumhalter verschlossenen Gefäße für 12 Stunden auf $60{ }^{\circ} \mathrm{C}$ im Trockenschrank. Nach dieser Zeit wurde auf $80^{\circ} \mathrm{C}$ aufgeheizt. Die Endtemperatur wurde für 4 Stunden gehalten. Der Aufschluß in Eppendorfgefäßen gestattet es, mit kleinen Einwaagen und kleinen Säuremengen arbeiten zu können. Die reproduzierbare hohe Reinheit der als Einmalgefäße im Handel verfügbaren Reaktionsgefäße gestattet eine Reduzierung des Aufwandes der Probenvorbereitung, da Gefäßvorreinigungen vollständig entfallen können (SPERLING, BAHR und KREMLING 1977).

Die so vollständig aufgeschlossenen Haarproben wurden nach dem Abkühlen mit $0,5 \mathrm{ml}$ Reinstwasser (MERCK, Art.-Nr. 1.16754) verdünnt und bis zur Analyse im Kühlschrank gelagert.

\subsubsection{Kotproben}

Die Kotproben wurden nach 12stündigem Trocknen bei $105^{\circ} \mathrm{C}$ in einer Reibschale zermahlen und homogenisiert. Anschließend erfolgte der Probenaufschluß mittels Königswasser gemäß DIN ISO 11466: 06.97.

\subsection{Bestimmung des Schwermetallgehaltes}

Die Bestimmung des Schwermetallgehaltes erfolgte mittels Atomabsorbtionsspektralphotometrie (AAS) und D2-Untergrundkompensation (Gerätetyp: SpectraA 20 der Firma Varian). Die Bestimmung von Kupfer und Zink in den Haarproben erfolgte mittels Flammen-AAS und Mikroinjektionstechnik. Die übrigen Schwermetalle in den Haar-, Knochen- und Gewebeproben wurden mittels Graphitrohr-AAS analysiert. Die Kalibrierung erfolgte über einen im Säuregehalt angepaßten Standardmix zu Beginn einer jeden Meßreihe. Die Bleigehalte in den Lungengewebeproben wurden mittels Standardadditionsverfahren ermittelt. Alle im nachfolgenden aufgeführten Meßwerte sind Mittelwerte aus einer Dreifachbestimmung. 
Die Kotproben wurden mittels ICP-OES (Gerätetyp: Spectroflame, Fa. Spectro) analysiert. Die Kalibrierung erfolgte unter Verwendung eines der Matrix angepaßten Standardmixes. Zur Korrektur des Plasmas wurde parallel zur eigentlichen Elementmessung das Argonsignal mit erfaßt. Zinn wurde mittels Graphitrohr-AAS bestimmt, Quecksilber und Arsen mittels Kaltdampf- bzw. Hydrid-AAS (Gerätetyp: VGA 76, Firma Varian). Die Bestimmung von Cadmium, dessen Elementlinie stark vom Calcium bei der ICP-OES-Messung gestört wird, wurde zusätzlich mittels Flammen-AAS ausgeführt.

\subsection{Statistische Methoden}

Die Berechnung der Verfahrenskenndaten (Anhang 3) der angewandten Meßverfahren erfolgte auf der Grundlage der Vorgaben der Deutschen Einheitsverfahren zur Wasser- Abwasser und Schlammuntersuchung (WASSERCHEMISCHE GESELLSCHAFT und NORMENAUSSCHUB WASSERWESEN 2000). Die Meßdaten der Kalibrierungen wurden in einem laborintern unter EXCEL 5.0 entwickelten Auswerteprogramm eingegeben und ausgewertet.

Eine grundsätzliche Bereinigung der Meßwerte mittels Ausreißertest nach GRUBBS erfolgte nicht, da gerade für Regionen mit hohen Schwermetallbelastungen positive Ausreißer, d.h. vereinzelte Tiere mit sehr hohen Schwermetallgehalten im Gewebe, typisch sind. Auch würde bei ausschließlicher Betrachtung ausreißerbereinigter Datensätze die Zuwanderung eines Tieres aus einer schwermetallbelasteten Region in eine unbelastete Region nicht mehr erkannt werden können.

Bei den Haaranalysen fanden sich vereinzelt offensichtliche Ausreißer. Diese sind gekennzeichnet durch hohe Zinkgehalte, die vermutlich auf eine exogene Verunreinigung der Haare zurückzuführen waren, die trotz der angewandten Reinigungsprozedur nicht entfernt werden konnten. Als solche Ausreißer wurden Meßwerte beurteilt, die größer oder gleich dem 95. Perzentil waren. Sofern solche Ausreißer vorlagen, wurde der gesamte Datensatz dieses Tieres nicht mit in die Auswertung einbezogen, da auch für die übrigen analysierten Elemente dann nicht ausgeschlossen werden konnte, daß eine exogene Verunreinigung für diese Elemente Mehrbefunde ergeben könnte. In den tabellarischen Auflistungen der Daten im Anhang sind diese Meßwerte der Vollständigkeit halber hingegen mit aufgeführt.

Zur Prüfung der Signifikanz eines Unterschiedes zweier Datensätze (z.B. Knochenbleigehalte in harzfernen Totfunden und Totfunden aus dem Harz) wurde der nichtparametrische U-Test nach MANN-WHITNEY angewandt, da es sich um nicht normalverteilte Datensätze handelte. Hierbei werden unter Berücksichtigung der Rangsummen zwei Datensätze miteinander verglichen. Die hieraus errechnete Prüfgröße wird mit Tabellenwerten zur Kennzeichnung der statistischen Sicherheit $(p)$ verglichen. Ist die Prüfgröße kleiner als die Kennzahl der statistischen Sicherheit von $95 \%$ $(>0,05)$, so gilt der beobachtete Unterschied der Medianwerte als zufällig. 
Ein wahrscheinlicher Unterschied liegt bei $p \geq 95 \%$ vor, ein signifikanter Unterschied gilt bei $p \geq 99 \%(=0,01)$. Als hochsignifikanter Unterschied werden Werte von $p \geq 99,9 \%(=0,001)$ betrachtet. Die Signifikanzprüfungen erfolgten unter Verwendung des Programmes STATISTICA. Die allgemeinen statistischen Kenndaten wurden mittels EXCEL 5.0 berechnet.

Die graphische Darstellung der Datensätze erfolgte in Form von Box-PlotDiagrammen unter Verwendung des mathematischen Programmpaketes StatPlot 5.0. Box-Plot-Diagramme bieten in komprimierter Form eine rasche Übersicht sowohl über den Medianwert eines Datensatzes als auch über den unteren und oberen Quartilswert (25\%-und $75 \%$-Quartile) sowie die Lage der $10 \%$ - und $90 \%$-Quantile. Meßwerte oberhalb und unterhalb dieser Grenzen werden durch Punkte dargestellt (Abb. 1).

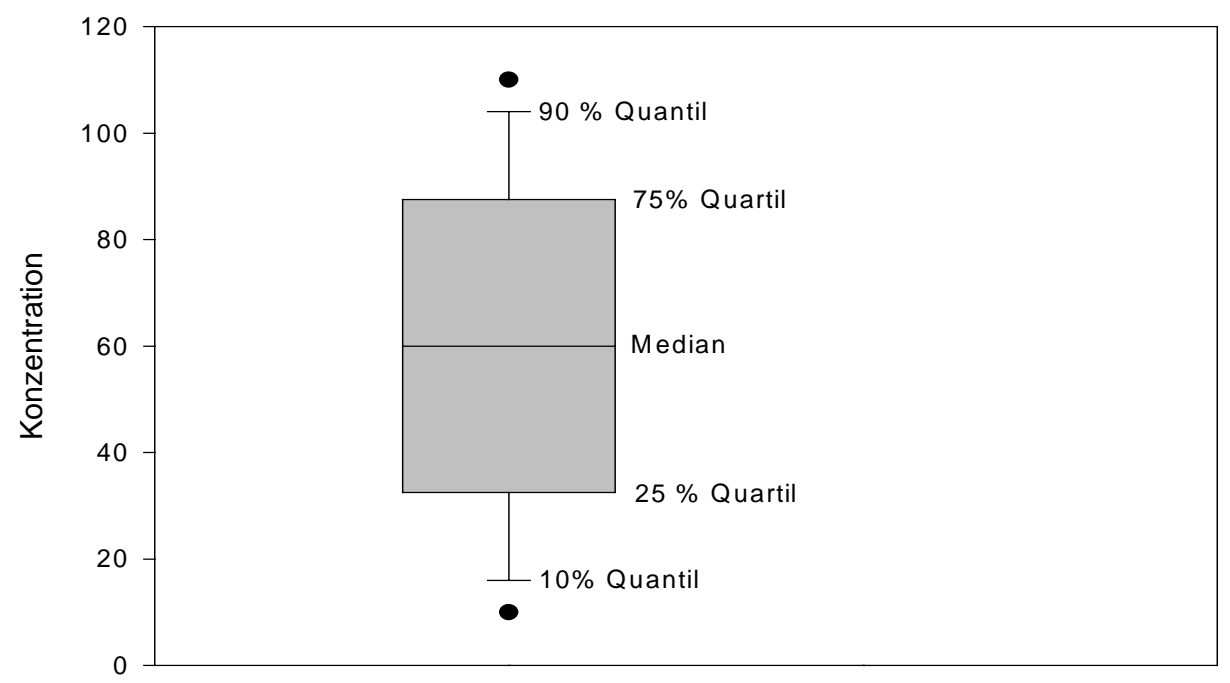

Abb. 1: Erläuterung einer Box-Plot-Darstellung. $50 \%$ alle Meßwerte befinden sich in dem Konzentrationsbereich, der durch einen grau hinterlegten Kasten dargestellt ist, d.h. in diesem Beispiel zwischen 32 und 88 Konzentrationseinheiten.

\section{Ergebnisse}

\section{1 Übersicht über die verfügbaren Fledermaustotfunde aus Niedersachsen}

Die Erfassung des vom Niedersächsischen Landesamt für Ökologie unter Mitarbeit zahlreicher ehrenamtlicher Fledermausschutzbeauftragter zusammengetragenen Tiermateriales ergab, daß für die Untersuchungen insgesamt 458 Individuen aus 13 Taxa in unterschiedlich guter Erhaltung zur Verfügung standen. Das Fundmaterial 
entstammte dem Zeitraum 1986 bis 1999, wobei Probenmaterial aus den vergangenen zehn Jahren überwog (Abb. 2). Aus dem Jahre 1986 waren nur zwei Totfunde (Wasserfledermaus, Braunes Langohr) vorhanden. Zwergfledermäuse (Pipistrellus pipistrellus) machten mit rund $55 \%$ der vorhandenen Tiere den überwiegenden Artenanteil aus (Abb. 3). In Niedersachsen kommen nach den Erhebungen des Landesamtes für Ökologie derzeit 17 Fledermausarten vor. Die für die Untersuchung zur Verfügung stehenden Tiere entstammten ungefähr zu gleichen Teilen aus dem Bereich des Harzes und aus dem übrigen Niedersachsen. Allerdings waren die einzelnen Spezies unterschiedlich verteilt (Tab. 6).

Ursache für diese Ungleichverteilung bei den Totfunden ist zum einen die unterschiedliche Populationsstärke der einzelnen in Niedersachsen vorkommenden Fledermausarten. Weitaus wesentlicher dürfte jedoch der Lebensraum der einzelnen Arten für die Häufigkeit der Totfunde sein. Während Zwergfledermäuse in besonderem $\mathrm{Maße}$ an menschliche Siedlungsgebiete angepaßt sind und dort bevorzugt in Spalten hinter Fassadenverkleidungen, auf Dachböden oder in Mauerspalten leben, bevorzugen Arten wie Teich- und Wasserfledermaus Lebensräume in Wäldern (vgl. Kap. 1.3). Die Wahrscheinlichkeit, daß eine verendete Fledermaus von Menschen gefunden wird, ist dabei in urbanen Lebensräumen weitaus höher als in siedlungsfernen Bereichen. Eine in einem Waldgebiet verendete Fledermaus wird vermutlich noch in derselben Nacht von einem Aasfresser verzehrt, wohingegen eine z.B. unter einer Straßenlaterne verendete Fledermaus im besiedelten Raum von Passanten noch am nächsten Morgen entdeckt werden kann.

Die ausgewerteten Totfunde dürfen somit nicht proportional der Abundanz der einzelnen Arten gesetzt werden.

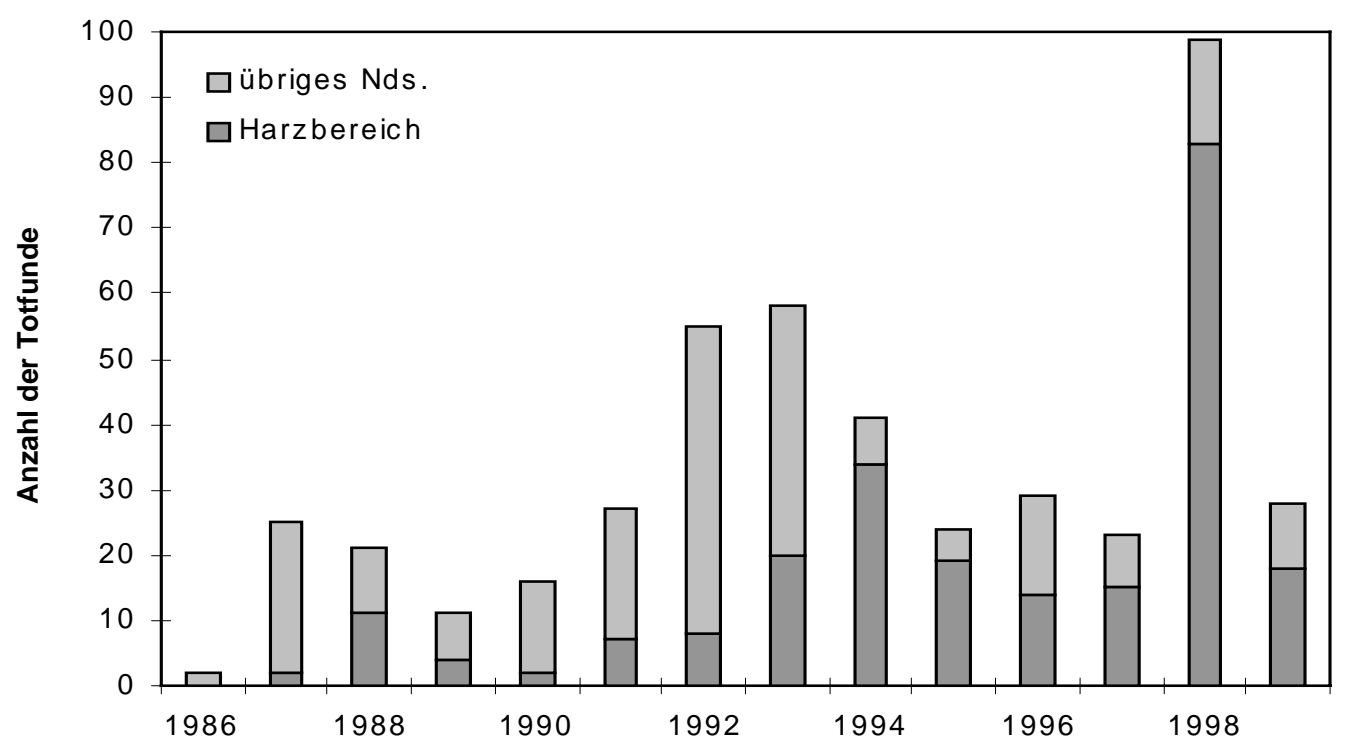

Abb. 2: Verteilung der Fledermaustotfunde aus Niedersachsen auf das jeweilige Jahr des Totfundes. 


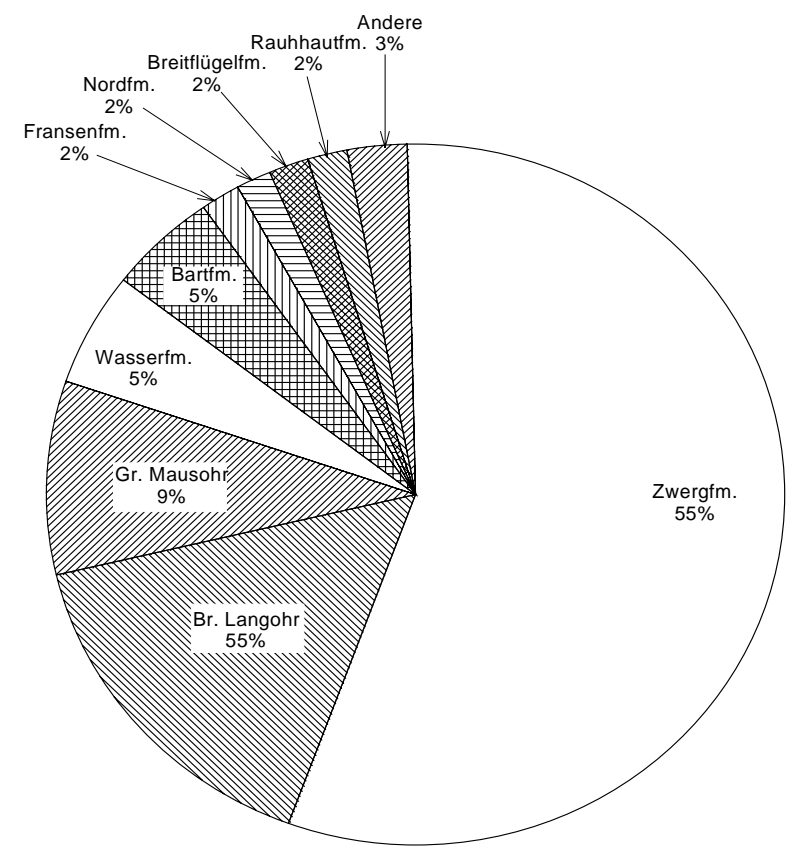

Abb. 3: Prozentuale Artenverteilung der verfügbaren Fledermaustotfunde aus den Jahren 1986 bis 1999 in Niedersachsen.

Tab. 6: Anzahl der verfügbaren Fledermaustotfunde aus Niedersachsen für die Jahre 1986 bis 1999

\begin{tabular}{lrrc}
\hline \hline \multicolumn{1}{c}{ Spezies } & $\begin{array}{c}\text { Tiere, } \\
\text { gesamt }\end{array}$ & $\begin{array}{c}\text { davon } \\
\text { Harz }\end{array}$ & $\begin{array}{c}\text { davon } \\
\text { harzfern }\end{array}$ \\
\hline Pipistrellus pipistrellus - Zwergfledermaus & 256 & 196 & 60 \\
Plecotus auritus - Braunes Langohr & 72 & 2 & 70 \\
Myotis myotis - Großes Mausohr & 41 & 10 & 31 \\
Myotis mystacinus - KI. Bartfledermaus & 22 & 12 & 10 \\
Myotis daubentonii - Wasserfledermaus & 24 & 0 & 24 \\
Myotis nattereri - Fransenfledermaus & 8 & 2 & 6 \\
Eptesicus nilssonii - Nordfledermaus & 7 & 7 & 0 \\
Eptesicus serotinus - Breitflügelfledermaus & 8 & 0 & 8 \\
Pipistrellus nathusii - Rauhhautfledermaus & 8 & 3 & 5 \\
Plecotus austriacus - Graues Langohr & 5 & 1 & 4 \\
Myotis bechsteinii - Bechsteinfledermaus & 2 & 2 & 0 \\
Nyctalus noctula - Großer Abendsegler & 3 & 2 & 1 \\
Nyctalus leisleri - Kl. Abendsegler & 2 & 0 & 2 \\
Myotis dasycneme - Teichfledermaus & 0 & 0 & 0 \\
Myotis brandtii - Gr. Bartfledermaus & 0 & 0 & 0 \\
Vespertilio murinus - Zweifarbfledermaus & 0 & 0 & 0 \\
Barbastella barbastellus - Mopsfledermaus & 0 & 0 & 0 \\
\hline Summe der verfügbaren 13 Arten: & $\mathbf{4 5 8}$ & $\mathbf{2 3 7}$ & $\mathbf{2 2 1}$ \\
\hline \hline
\end{tabular}




\subsection{Zum Vorkommen der Mückenfledermaus}

Aufgrund der im Jahre 1993 entdeckten Unterschiede in der Ruffrequenz bei verschiedenen Zwergfledermäusen wird seit einigen Jahren das Vorkommen einer zweiten, sympatrisch lebenden Zwergfledermausart diskutiert. Während die Stammform der Zwergfledermaus eine Ruffrequenz um $45 \mathrm{kHz}$ besitzt, ruft eine andere Gruppe mit einer Ruffrequenz um $55 \mathrm{kHz}$. Nachdem in den zurückliegenden Jahren mehrere Untersuchungen zur Artdifferenzierung der beiden Zwergfledermausgruppen erfolgten, publizierten HäUSSLER et al. (2000) erstmals einen Bestimmungsschlüssel zur Unterscheidung der beiden Gruppen. Die mit einer Ruffrequenz von $55 \mathrm{kHz}$ vorkommende Form wurde mit dem Artnamen Pipistrellus pygmaeus - Mückenfledermaus belegt. Nach Einschätzung von HÄUSSLER et al. (2000) belegen sowohl genotypische Untersuchungen als auch Untersuchungen zum Verhalten und zur ökologischen Anpassung in ausreichendem Maße, daß es sich hier um zwei verschiedene Arten handeln muß. Anhand der bei HäUSSLER et al. publizierten wesentlichen Differenzierungsmerkmale (Tab. 7) erfolgte nach Abschluß der eigentlichen Laboruntersuchung nochmals eine Durchsicht der im Rahmen der Schwermetalluntersuchungen bearbeiteten Zwergfledermäuse.

Tab. 7: Wesentliche Artmerkmale zur Differenzierung von Zwergfledermaus ( $P$. pipistrellus) und Mückenfledermaus ( $P$. pygmaeus), nach HäUSSLER et al. 2000

\begin{tabular}{|c|c|c|}
\hline Merkmal & $\begin{array}{l}\text { Zwergfledermaus } \\
\text { (P. pipistrellus) }\end{array}$ & $\begin{array}{l}\text { Mückenfledermaus } \\
\text { (P. pygmaeus) }\end{array}$ \\
\hline Ortungsruf & um $45 \mathrm{kHz}$ & um 55 kHz \\
\hline Schwanzflughaut & spärlich behaart & körpernah deutlich behaart \\
\hline Flughauthinterrand & $\begin{array}{l}\text { zwischen } 5 \text {. Finger und Hinterfuß sel- } \\
\text { ten hell }\end{array}$ & $\begin{array}{l}\text { zwischen 5. Finger und Hinterfuß } \\
\text { deutlich hell gefärbt }\end{array}$ \\
\hline Penis & $\begin{array}{l}\text { schiefergrau mit deutlicher weißer } \\
\text { Längslinie in der Mitte der Unterseite }\end{array}$ & nahezu einfarbig rot-braun \\
\hline Fingerglieder & $\begin{array}{l}\text { 2. und 3. Glied des } 3 \text {. Fingers sind } \\
\text { unterschiedlich lang }\end{array}$ & $\begin{array}{l}\text { 2. und 3. Glied des } 3 \text {. Fingers sind } \\
\text { gleich lang }\end{array}$ \\
\hline
\end{tabular}

Die Auswertung der gemessenen biometrischen Daten von insgesamt 70 der untersuchten Zwergfledermäuse ergaben bei 16 Tieren (23 \%) einen Quotienten zwischen der Länge des Fingergliedes II und III des 3 . Fingers von $\leq 1,05$. Von diesen 16 Tieren waren 6 Tiere $(=9 \%)$ juvenil und weitere 5 Tiere $(7 \%)$ subadult. Das für die Mückenfledermaus geltende Merkmal „Fingerglied II gleichlang dem Fingerglied III“ traf lediglich auf 5 adulte Exemplare $(=7 \%)$ zu. Eine deutlich orange bis rotbraune Färbung des Penis fand sich bei zwei Tieren (Tier-Nr. 95014 und 98066), deren Fingergliedlängenverhältnis jedoch deutlich unterschiedlich waren. 
Der graphische Vergleich zwischen der Länge des Fingergliedes II und III zeigt deutlich, daß bei kürzeren Knochenlängen, d.h. jüngeren Tieren, das Verhältnis gegen 1 tendiert. Erst bei ausgewachsenen Tieren ist die Länge des Fingergliedes II des 3. Fingers von P. pipistrellus signifikant länger als die des Fingergliedes III (Abb. 4). Die Auflistung sämtlicher Meßwerte befindet sich im Anhang 1.

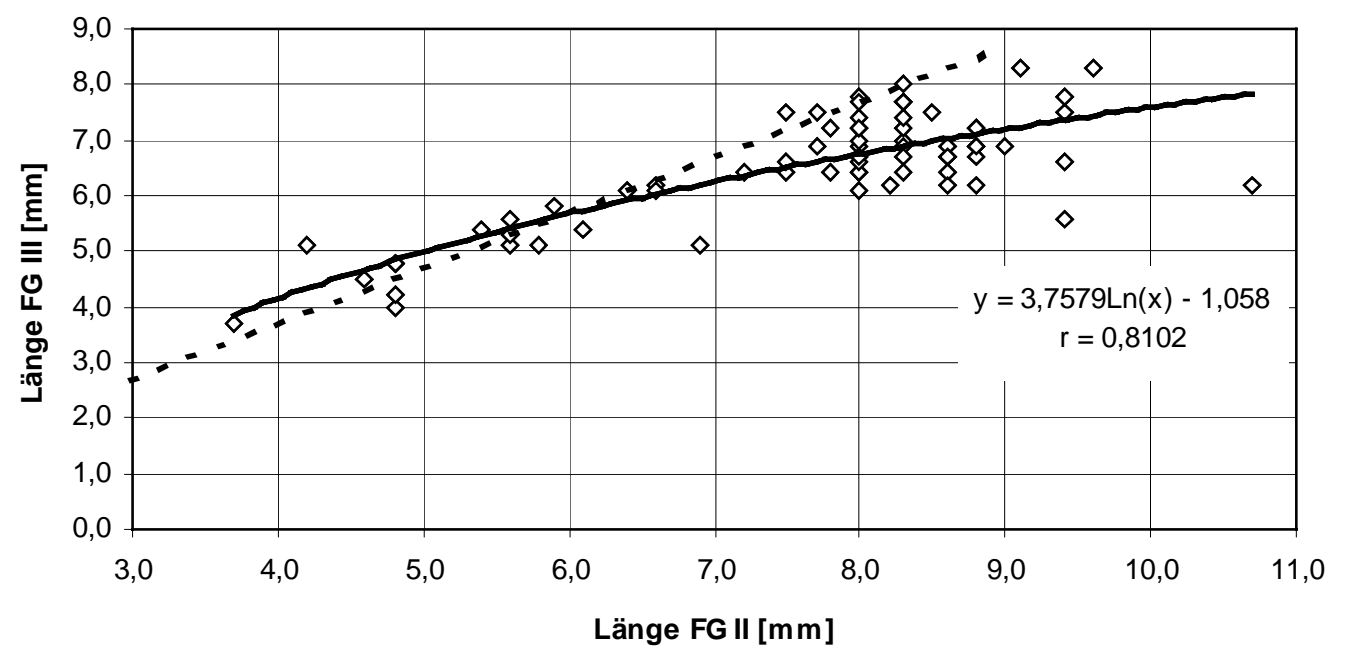

Abb. 4: Korrelation zwischen der Länge des Fingergliedes II und Fingergliedes III des 3. Fingers bei der Formengruppe Pipistrellus pipistrellus. Die durchgezogene Linie stellt die Regressionskurve aller Meßwerte dar, die gestrichelte Linie entspricht der Ideallinie für die Mückenfledermaus, d.h. die Fingerglieder II und III des 3 . Fingers sind gleich lang. Deutlich ist erkennbar, daß dieses für die Mückenfledermaus geltende Artdifferenzierungsmerkmal gleichfalls auf alle untersuchten Jungtiere der beiden Formengruppen zutrifft.

Die fünf adulten Tiere mit einem ungefähr gleichen Längenverhältnis der beiden Fingerglieder von rund 1:1 (Fingerglied II/Fingerglied III $\leq 1,05$ ) wurden ergänzend vermessen und unter dem Stereomikroskop untersucht. Die Ergebnisse dieser Detailuntersuchung zeigen, daß die für die Mückenfledermaus beschriebenen Merkmale nicht eindeutig sind. Es fanden sich sowohl Männchen mit grauem Penis und hellem Streifen bei ungefähr gleichem Längenverhältnis der beiden Fingerglieder (Tier-Nr. 98070) als auch Tiere mit rotbraunem Penis ohne Längsstreifen, aber deutlich unterschiedlichem Längenverhältnis der beiden Fingerglieder. Auch hinsichtlich der beschriebenen Farbunterschiede im Fell konnten die Kriterien dunkelbraun und olivbraun unabhängig vom Längenverhältnis der Fingerglieder beobachtet werden (Tab. 8). 
Tab. 8: Ergebnisse der morphologischen und biometrischen Detailuntersuchung an adulten Zwergfledermäusen mit einem annähernd gleichen Längenverhältnis der Fingerglieder II/III

\begin{tabular}{|c|c|c|c|c|c|}
\hline Tier-Nr. & 94015 & 95012 & 98014 & 98070 & 99006 \\
\hline Geschlecht: & w & w & $w$ & $\mathrm{~m}$ & w \\
\hline Unterarmlänge [mm]: & 31,4 & 29,5 & 29,7 & 31,5 & 31,0 \\
\hline $\begin{array}{l}\text { Länge Fingerglied II, Fin- } \\
\text { ger } 3 \text { [mm]: }\end{array}$ & 8,0 & 7,7 & 6,6 & 8,0 & 8,3 \\
\hline $\begin{array}{l}\text { Länge Fingerglied III, } \\
\text { Finger } 3[\mathrm{~mm}] \text { : }\end{array}$ & 7,8 & 7,5 & 6,2 & 7,7 & 8,0 \\
\hline FGII - FG III [mm]: & 0,2 & 0,2 & 0,3 & 0,3 & 0,3 \\
\hline $\begin{array}{l}\text { Länge Mittelhandkno- } \\
\text { chen, Finger } 5[\mathrm{~mm}]:\end{array}$ & 26 & 24,5 & 24 & 26 & 26,4 \\
\hline $\begin{array}{l}\text { Gesamtlänge Finger } 5 \\
\text { [mm]: }\end{array}$ & 38,2 & 36 & 34,8 & 36,7 & 37,6 \\
\hline Nasenbreite $[\mathrm{mm}]$ : & 2,5 & 2,8 & 2,2 & 3,0 & 3,0 \\
\hline Rückenfell: & $\begin{array}{l}\text { kastanienbraun, Unterfell } \\
\text { grauschwarz }\end{array}$ & $\begin{array}{l}\text { olivbraun, Unterfell grau- } \\
\text { schwarz }\end{array}$ & $\begin{array}{l}\text { olivbraun, Unterfell grau- } \\
\text { schwarz }\end{array}$ & $\begin{array}{l}\text { kastanienbraun, Unterfell } \\
\text { grauschwarz }\end{array}$ & $\begin{array}{l}\text { kastanienbraun, Unterfell } \\
\text { grauschwarz }\end{array}$ \\
\hline $\begin{array}{l}\text { Färbung Flügelhinterrand } \\
\text { zw. Finger } 5 \text { und Fuß: }\end{array}$ & $\begin{array}{l}\text { mit schmalen hellen } \\
\text { Streifen }\end{array}$ & deutl. weißer Streifen & $\begin{array}{l}\text { dünner weißer Streifen. } \\
\text { Flügeldecken intensiv } \\
\text { schwarz }\end{array}$ & & $\begin{array}{l}\text { schmaler hellgrauer } \\
\text { Streifen }\end{array}$ \\
\hline Penis: & - & - & - & $\begin{array}{l}\text { grau, mit medialen, hel- } \\
\text { len Streifen im vorderen } \\
\text { Drittel }\end{array}$ & - \\
\hline
\end{tabular}


Aufgrund der Ergebnisse der biometrischen Datenerhebung und der ergänzenden morphologischen Untersuchung der stichprobenartig ausgewählten 70 Tiere kann für das untersuchte Tiermaterial festgestellt werden, daß Mückenfledermäuse $(P$. pygmaeus) nicht in dem vorhandenen Fundmaterial enthalten sind. Im Nachfolgenden werden daher alle untersuchten Tiere des Formenkreises "Zwergfledermaus“ als $P$. pipistrellus behandelt.

\subsection{Bestimmung von Umrechnungsfaktoren zur Normierung anderer Untersuchungsergebnisse von Organproben auf die Trockensubstanz}

In der Literatur finden sich häufig Analysenwerte, deren Konzentrationsangaben auf unterschiedlichen Bezugsgrößen fussen. Oftmals bleibt es dabei auch dem Leser überlassen, für welche Bezugsgröße er sich entscheidet.

Es ist leicht nachvollziehbar, daß der auf die Probeneinwaage umgerechnete Meßwert in Abhängigkeit vom Feuchtegehalt der Analysenprobe stark differiert. Die Leber einer frischtoten, noch bluterfüllten Fledermaus wiegt etwa $200 \mathrm{mg}$ (P. pipistrellus). In teilweise mumifizierten Tierleichen beträgt das Gewicht der Leber infolge Flüssigkeitsverlust deutlich weniger, wobei in Abhängigkeit vom Mumifizierungsgrad eine allmähliche Gewichtsabnahme erfolgt. Die gefriergetrocknete Leber hat nur noch ein Gewicht zwischen etwa $20 \mathrm{mg}$ und $40 \mathrm{mg}$. Ohne Festlegung des eigentlichen Meßergebnisses auf die Trockensubstanz der Probeneinwaage würden sich somit Schadstoffkonzentrationen berechnen, die wesentlich vom Mumifizierungsgrad der Tiere abhängig sind, wodurch keine gesicherte Rückführung auf die tatsächliche Schadstoffbelastung des untersuchten Tieres und kein Vergleich zwischen den Probenkollektiven mehr möglich wäre.

Im Rahmen der durchgeführten Gefriertrocknung der Gewebeproben wurde sowohl das Frischgewicht als auch das Trockengewicht einer jeden Probe bestimmt. Auf dieser Datengrundlage lassen sich die nachfolgenden Kennzahlen des mittleren Feuchtegehaltes von Gewebeproben aus Fledermäusen berechnen, die zur Abschätzung des auf die Trockensubstanz (TS) bezogenen Meßwertes dienen können, sofern sich in der Originalliteratur nur Feuchtsubstanzangaben (FS) finden. Für die verschiedenen Organe frischtoter Tiere errechnete sich folgender mittlerer Wassergehalt $( \pm 1 \mathrm{~s})$ :

$\begin{array}{lll}\text { Leber }(n=17): & 73,2 \pm 0,96 & \text { Gew.- } \% \\ \text { Nieren }(n=19): & 74,2 \pm 0,96 & \text { Gew.- } \% \\ \text { Lunge }(n=13): & 76,5 \pm 1,21 & \text { Gew.- } \% \\ \text { Herz }(n=20): & 72,8 \pm 0,76 & \text { Gew.- } \% .\end{array}$


Hieraus lassen sich folgende Umrechnungsfaktoren abschätzen:

\begin{tabular}{lcc}
\hline \hline \multicolumn{1}{c}{ Gewebe } & FS $\rightarrow$ TS & TS $\rightarrow$ FS \\
\hline Leber & 3,7 & 0,27 \\
Nieren & 3,9 & 0,26 \\
Lunge & 4,3 & 0,23 \\
Herz & 3,7 & 0,27 \\
\hline \hline
\end{tabular}

\subsection{Wasseraufnahmevermögen des Fledermaushaares}

Getrocknete, wasserfreie Haare nehmen nach BRAUKHOFF (1944) bei $100 \%$ relativer Luftfeuchtigkeit bis zu 31,2\% des Eigengewichtes an Wasser auf. Aufgrund dieser hygroskopischen Eigenschaften von Haaren können Messungen zu verschiedenen Jahreszeiten infolge der differierenden relativen Feuchtigkeit der Laborluft zu signifikanten Unterschieden im Befund und damit zu Fehlbeurteilungen führen (GIBSON et al. 1989). Eigene Versuche mit Haarproben von Zwergfledermäusen zeigten, daß sich diese Haare im Wasseraufnahmevermögen analog zum Humanhaar verhalten (Abb. 5). Ein hiervon abweichendes Wasseraufnahmevermögen wies hingegen eine Haarprobe vom Braunen Langohr auf. Die Einstellung des Wassergehaltes im Haar verläuft dabei relativ rasch. Offen auf einer Petrischale liegende Haare erreichen nach etwa 30 min. ein Gleichgewicht mit der Umgebungsfeuchte. Die tabellarische Auflistung der Meßwerte befindet sich im Anhang 2.

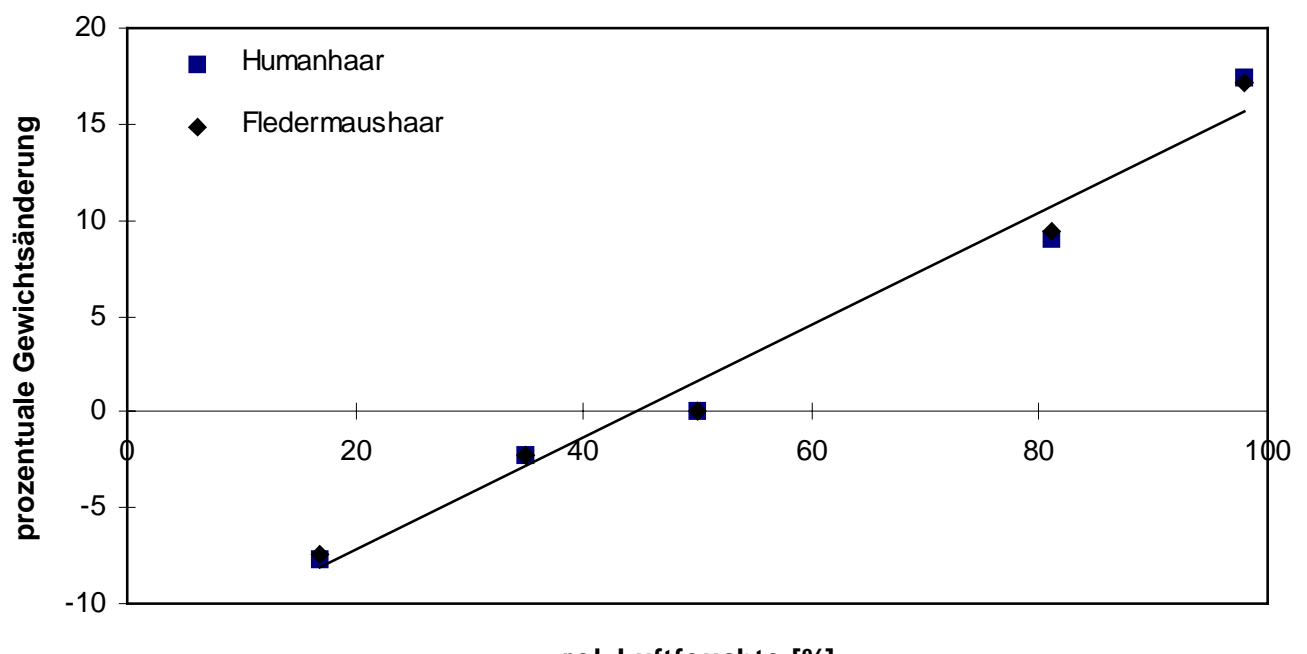

rel. Luftfeuchte [\%]

Abb. 5: Relative Gewichtsänderung von Humanhaar und Zwerfledermaushaar bei unterschiedlicher relativer Luftfeuchtigkeit. Die Äquilibrierungszeit betrug jeweils mindestens 24 Stunden. 
Der annähernd identische Verlauf des Wasseraufnahmevermögens der Fledermaushaare mit dem der Humanhaare ist wenig verwunderlich, da der prinzipielle chemische und strukturelle Aufbau bei Säugetierhaaren ähnlich ist. Lediglich die morphologische Ausbildung (Durchmesser, Länge, Oberflächenstruktur) sowie die Ausbildung eines Markstranges variiert artabhängig, teilweise sogar rassenabhängig. Nach KATZ und CHATT (1988) besteht das menschliche Haar zu rund $80 \%$ aus Proteinen und zu rund $15 \%$ aus Wasser. Der Mineralstoffanteil, bezogen auf das Aschegewicht, beträgt $0,25 \%$ bis $0,95 \%$.

Der Wassergehalt des Haares steht in unmittelbarem Bezug zur umgebenden Luftfeuchtigkeit. Dieser Umstand wurde bereits im 18. Jahrhundert entdeckt und führte zum Bau der noch heute verwendeten Haarhygrometer zur Bestimmung der Luftfeuchtigkeit.

Die ermittelten Änderungen des Haaregewichtes in Abhängigkeit von der umgebenden Luftfeuchtigkeit decken sich mit den Angaben von RoBBINS (1988). Dieser ermittelte für Humanhaar bei einer Erhöhung der Luftfeuchtigkeit um $30 \%$ einen Anstieg des Wassergehaltes im Haar um rund $6 \%$. Die eigenen Untersuchungen am Human- und Fledermaushaar ergaben Änderungen des Wassergehaltes von $9 \%$ innerhalb eines Feuchtebereiches von $30 \%$ rel. Luftfeuchte.

Luftfeuchtigkeitsänderungen von $30 \%$ rel. Feuchte sind im Jahresgang in nicht klimatisierten mitteleuropäischen Laborräumen durchaus üblich. Zur Erzeugung vergleichbarer Meßwerte des Mineralstoffgehaltes im Haar ist daher die Berücksichtigung und Angabe des Feuchtegehaltes im Haar oder der Bezug auf das Aschegewicht des Haares unerläßlich.

\subsection{Qualitätssichernde Maßnahmen der Rückstandsanalytik}

\subsubsection{Kalibrierung}

Zur Absicherung der Richtigkeit und Präzision der Meßergebnisse wurde zu Beginn der Meßreihen eine 10-Punkt-Kalibrierung über das Gesamtverfahren durchgeführt. Die aus diesen Daten errechneten Verfahrensvariationskoeffizienten und Verfahrenskenndaten finden sich im Anhang 3 dieser Arbeit. Die Ermittlung der Verfahrenskenndaten wurde in regelmäßigen Abständen, etwa alle 12 Monate, wiederholt. Darüber hinaus wurde u.a. an bundesweiten Ringversuchen jeweils mit Erfolg teilgenommen.

Zur Absicherung der Richtigkeit wurde ferner zertifiziertes Referenzmaterial in analoger Weise wie das Probenmaterial aufgeschlossen und gemessen. Zur Anwendung kam dabei folgendes Referenzmaterial:

- BCR-Referenzmaterial CRM 397 „Human-hair“, Lot No. 213

- NIST-Referenzmaterial SRM 1400 „Bone ash“. 
Die Bestimmung der Schwermetallgehalte an dem Humanhaar-Referenzmaterial erfolgte unter Verwendung von jeweils etwa $30 \mathrm{mg}$ Referenzmaterial. Diese $30 \mathrm{mg}$ entsprechen in etwa den von Fledermäusen gewinnbaren Haarprobenmengen. Die Probeneinwaagen lagen damit etwa $1 / 3$ bis $1 / 30$ niedriger als bei den Bestimmungen im Rahmen der Zertifizierung des Referenzmateriales.

Analog wurde bei der Bestimmung des Knochenbleigehaltes am Referenzmaterial verfahren. Auch hier wurde eine Probeneinwaage gewählt, die dem gewinnbaren Probenmaterial von Fledermäusen entsprach. Die Probeneinwaage lag hier um $20 \mathrm{mg}$ und unterschritt damit die vorgegebene Mindesteinwaage zur Untersuchung des Referenzmateriales von $150 \mathrm{mg}$ deutlich. Die Bestimmungen ergaben für Blei im bone-ash-Referenzmaterial einen Mehrbefund von 3,85\% gegenüber dem zertifizierten Wert. Unter Berücksichtigung der gewählten deutlich niedrigeren Einwaage wird diese Abweichung für akzeptabel erachtet. Sie liegt damit zudem nur geringfügig oberhalb des im Rahmen der Überprüfung der Verfahrenskenndaten ermittelten Verfahrensvariationskoeffizienten für Blei (Tab. 9). Für das untersuchte human-hairReferenzmaterial lagen die ermittelten Gehalte für Cadmium und Kupfer rund $2 \%$ unter den zertifizierten Werten. Für Blei und Zink fanden sich deutliche Mehrbefunde von $15 \%$ (Blei) bzw. $13 \%$ (Zink). Da die Überprüfung der Verfahrensblindwerte keine Hinweise auf Kontaminationen bei der Probenaufbereitung ergab, dürfte die Ursache für die Mehrbefunde beim Blei primär eine matrixbedingte Störung bei der Atomisierung sein. Diese konnte auch durch Verwendung verschiedener Matrixmodifier (Ammonium-di-hydrogenphosphatmodifier und Palladiummodifier) nicht verringert werden. Gleiche Probleme bei der Bestimmung lagen möglicherweise auch bei den zertifizierenden Laboratorien im Rahmen der Ermittlung der „wahren“ Elementgehalte des Referenzmaterials vor. Alle drei Laboratorien (von 16), die die Bleibestimmung mittels Graphitrohr-AAS ausführten, erzielten Meßwerte, die mit am stärksten vom zertifizierten Sollwert abwichen. Die Meßwerte (Mittelwerte aus 5 bis 6 Einzelmessungen) der mittels Graphitrohr-AAS zertifizierenden Laboratorien wichen um $-9,1 \%,+7,2 \%$ und $+12,1 \%$ vom zertifizierten Referenzwert ab. Unter Berücksichtigung der bei den eigenen Messungen deutlich geringeren Probeneinwaage und des miniturisierten Aufschlußverfahrens kann daher die $15 \%$ ige Abweichung der eigenen Meßwerte vom zertifizierten Sollwert noch akzeptiert werden.

Proben mit auffallend hohen Meßwerten wurden zur Befundabsicherung zudem in einer späteren Meßserie stichprobenartig erneut gemessen. 
Tab. 9: Vergleich der zertifizierten und informativen Werte des Referenzmateriales „Human hair" und „Bone ash" mit den gemessenen Werten

a) Human hair:

\begin{tabular}{lccc}
\hline \hline \multicolumn{1}{c}{ Element } & Einheit & zertifizierter Gehalt & ermittelter Gehalt \\
\hline Blei & $\mu \mathrm{g} / \mathrm{g}$ TS & $33 \pm 1,2$ & $38 \pm 0,85$ \\
Cadmium & $\mu \mathrm{g} / \mathrm{g}$ TS & $0,521 \pm 0,24$ & $0,51 \pm 0,014$ \\
Kupfer & $\mu \mathrm{g} / \mathrm{g}$ TS & $110 \pm 5$ & $108 \pm 20$ \\
Zink & $\mu \mathrm{g} / \mathrm{g}$ TS & $199 \pm 5$ & $225 \pm 6,5$ \\
\hline$\left.{ }^{*}\right)$ informativer Wert & & & \\
b) Bone ash: & & & \\
\hline$\quad$ Element & Einheit & zertifizierter Gehalt & ermittelter Gehalt \\
\hline Blei & $\mu \mathrm{g} / \mathrm{g}$ TS & $9,07 \pm 0,12$ & $9,42 \pm 0,45$ \\
\hline \hline
\end{tabular}

\subsubsection{Abhängigkeit des Meßwertes von der Probeneinwaage}

Aufgrund der zur Verfügung stehenden geringen Probenmengen wurde anhand des Knochenasche-Referenzmateriales überprüft, inwieweit die Probenmenge einen Einfluß auf den Meßwert hat. Gewählt wurden Probeneinwaagen zwischen etwa $15 \mathrm{mg}$ und $30 \mathrm{mg}$. Die Messungen ergaben dabei keinen erkennbaren Zusammenhang zwischen der Probeneinwaage und der Wiederfindung (Abb. 6).

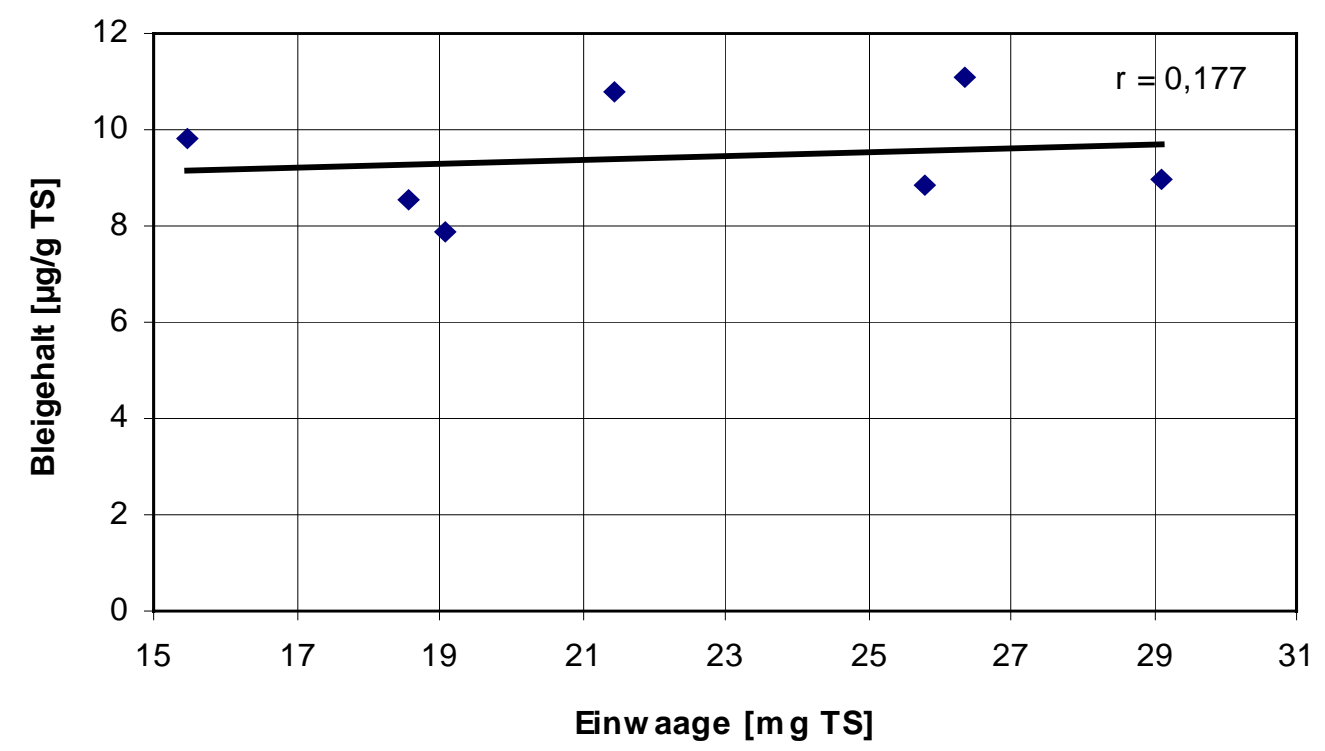

Abb. 6: Abhängigkeit des Meßwertes von der Probeneinwaage am Beispiel der Bleibestimmungen am „Bone-ash“-Referenzmaterial. 


\subsubsection{Variation der Knochenbleigehalte innerhalb eines Individuums}

Die Knochenbleigehalte in Säugetieren unterliegen abhängig vom Grad der Ossifikation und der damit einhergehenden Änderungen des Calciumgehaltes im Knochen deutlichen Schwankungen (BARRY 1975). Zur Gewährleistung der Vergleichbarkeit der Meßergebnisse wurden im Rahmen dieser Untersuchungen daher nur die Unterarmknochen verwendet. Signifikante Abweichungen im Knochenbleigehalt verschiedener Probenaufschlüsse von einem Individuum würden auf exogene Kontaminationen bei der Probenaufbereitung oder größere Meßfehler bei der eigentlichen Bestimmung hinweisen. Von zehn Tieren wurden zu unterschiedlichen Terminen sowohl der linke als auch der rechte Unterarm herauspräpariert, aufgeschlossen und gemessen. Die Unterschiede dieser Doppelbestimmungen betrugen im Mittel um 8,5\%, wobei die prozentuale Abweichung bei geringen Einwaagemengen (Knochen von Jungtieren) naturgemäß höher lag (Tab. 10).

Tab. 10: Ergebnisse von Doppelbestimmungen des Knochenbleigehaltes in linkem und rechtem Unterarm von P. pipistrellus und M. myotis

\begin{tabular}{|c|c|c|c|c|}
\hline Tier-Nr. & Artname & 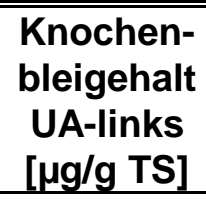 & $\begin{array}{l}\text { Knochen- } \\
\text { bleigehalt } \\
\text { UA-rechts } \\
\text { [ } \mu \mathrm{g} / \mathrm{g} \text { TS] }\end{array}$ & $\begin{array}{c}\text { Prozentuale Ab- } \\
\text { weichung vom } \\
\text { Mittelwert }\end{array}$ \\
\hline 87010 & P. pipistrellus & 4,2 & 7,8 & 30 \\
\hline 88009 & P. pipistrellus & 21 & 22 & 2,3 \\
\hline 93052 & P. pipistrellus & 5,5 & 6,3 & 6,8 \\
\hline 98035 & P. pipistrellus & 143 & 129 & 5,1 \\
\hline 98038 & P. pipistrellus & 11 & 13 & 8,3 \\
\hline 98040 & P. pipistrellus & 37 & 35 & 2,8 \\
\hline 98041 & P. pipistrellus & 17 & 17 & 0,0 \\
\hline 98042 & P. pipistrellus & 68 & 64 & 3,0 \\
\hline 93003 & M. myotis & 9,2 & 6,6 & 16 \\
\hline 93013 & M. myotis & 2,8 & 3,5 & 11 \\
\hline
\end{tabular}

\subsection{Bleigehalte in Knochen}

\section{Entwicklung der Knochenbleigehalte im Zeitraum 1987 bis 1999:}

Die mittleren Knochenbleigehalte nahmen im Untersuchungszeitraum von 1987 bis 1999 für Zwergfledermäuse in harzfernen Bereichen kontinuierlich ab (Abb. 7). Gegenüber den Knochenbleigehalten der Totfunde aus dem Zeitraum 1987 bis 1990 lag der Knochenbleigehalt rund zehn Jahre später $50 \%$ niedriger. Dieser Trend kann für Zwergfledermäuse, die im Harzbereich gefunden wurden, nicht bestätigt werden. Deren mittlere Knochenbleibelastung entspricht der Belastung von Tieren aus harzfernen Bereichen für den Betrachtungszeitraum 1987-1990 (Tab. 11). 

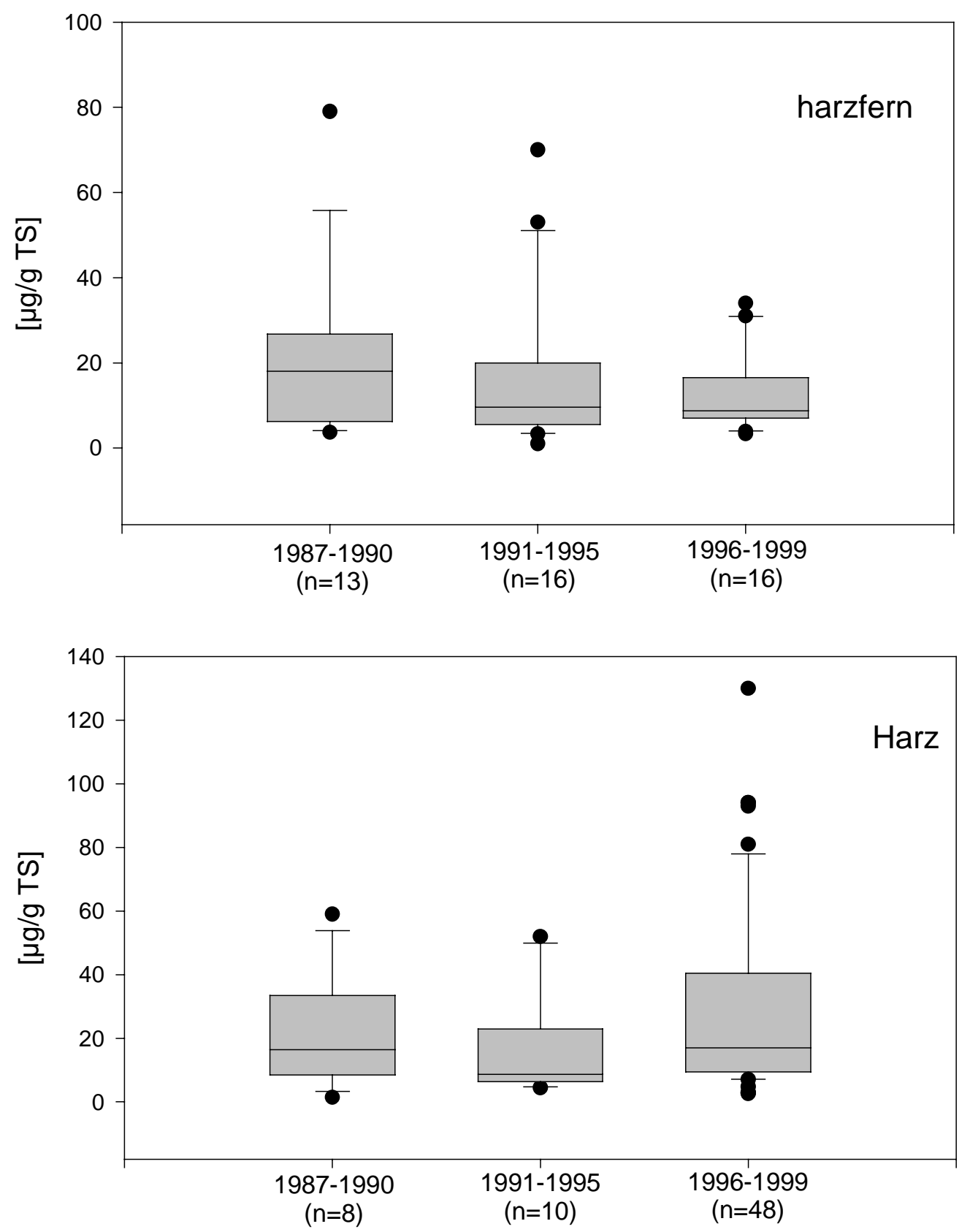

Abb. 7: Zeitlicher Verlauf der Knochenbleigehalte in Zwergfledermäusen ( $P$. pipistrellus) im Zeitraum 1987 bis 1999. 
Tab. 11: Medianwerte $[\mu \mathrm{g} / \mathrm{g}$ TS] der Knochenbleigehalte in Zwergfledermäusen ( $P$. pipistrellus) im Zeitraum 1987 bis 1999 und Signifikanz (p) des Unterschiedes der Knochenbleigehalte beider Gruppen (U-Test nach Mann-Whitney)

\begin{tabular}{cccc}
\hline \hline Betrachtungszeitraum & Totfunde harzfern & Totfunde Harz & $\mathbf{p}$ \\
\hline $1987-1990$ & $18(n=13)$ & $16(n=8)$ & 0,717 \\
$1991-1995$ & $9,6(n=16)$ & $8,7(n=10)$ & 0,345 \\
$1996-1999$ & $8,8(n=16)$ & $17(n=48)$ & 0,012 \\
\hline $1987-1999$ & $9,7(n=45)$ & $16(n=66)$ & 0,017 \\
\hline \hline
\end{tabular}

Der Unterschied der Knochenbleigehalte im Zeitraum 1996-1999 wird noch deutlicher bei der Zuordnung der Meßwerte zu einzelnen Klassen und bei der Betrachtung der Verteilung auf die einzelnen Klassen (Abb. 8). Die im Bereich des Harzes gefundenen Tiere haben nicht nur einen signifikant erhöhten Medianwert des Knochenbleigehaltes im Vergleich zu Totfunden aus dem übrigen Niedersachsen, auch ist die Verteilung der ermittelten Bleigehalte deutlich ungleichförmiger und rechtsschiefer (Tab. 12).
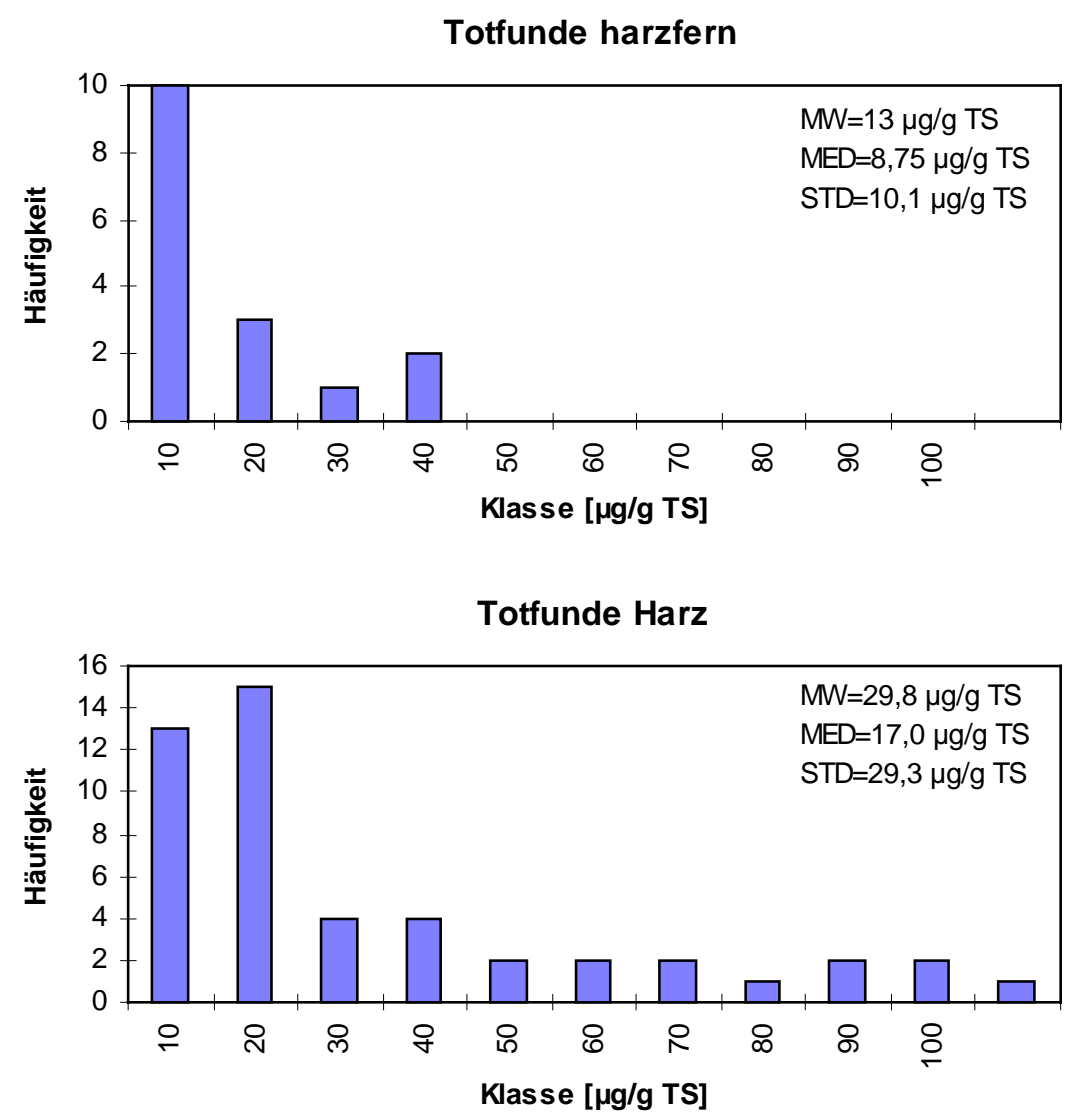

Abb. 8: Histogramme der Knochenbleigehalte in Zwergfledermäusen ( $P$. pipistrelIUS) aus dem Zeitraum 1996-1999. 
Tab. 12: Statistische Kenngrößen der Knochenbleigehalte in Zwergfledermäusen ( $P$. pipistrellus) aus dem Zeitraum 1996 bis 1999

\begin{tabular}{|c|c|c|}
\hline & "harzfern & Harz \\
\hline Stichprobenumfang & 16 & 48 \\
\hline Mittelwert $[\mu \mathrm{g} / \mathrm{g}$ TS] & 13 & 30 \\
\hline Median $[\mu \mathrm{g} / \mathrm{g}$ TS] & 8,8 & 17 \\
\hline Standardabweichung & 10,08 & 29,34 \\
\hline Schiefe & 1,28 & 1,55 \\
\hline Spannweite $[\mu \mathrm{g} / \mathrm{g}$ TS] & 30,7 & 127,4 \\
\hline Minimum $[\mu \mathrm{g} / \mathrm{g}$ TS] & 3,3 & 2,6 \\
\hline Maximum $[\mu \mathrm{g} / \mathrm{g}$ TS] & 34 & 130 \\
\hline
\end{tabular}

\section{Abhängigkeit vom Alter:}

Die Unterarmlänge der Tiere, bei adulten Exemplaren ein wichtiges Artunterscheidungsmerkmal, ist gleichfalls abhängig vom Alter der Tiere (Tab. 13). Bei Korrelation der Unterarmlänge mit dem Knochenbleigehalt ist somit eine Aussage der altersabhängigen Bleibelastung im Organismus möglich. Aufgrund der bereits beschriebenen starken Streuung der Meßwerte für den Knochenbleigehalt bei Tieren aus dem Harz war eine interpretationsfähige Korrelation nur bei harzfernen Funden möglich.

Tab. 13: Zuordnung der ungefähren Altersgruppe anhand der Unterarmlänge [cm] für die beiden Arten Zwergfledermaus ( $P$. pipistrellus) und Großes Mausohr (M. myotis)

\begin{tabular}{ccc}
\hline \hline Altersgruppe & P. pipistrellus & M. myotis \\
\hline juvenil & $<2,6$ & $<3,5$ \\
subadult & $2,6-3,1$ & $3,5-4,8$ \\
adult & $>3,1$ & $>4,8$ \\
\hline \hline
\end{tabular}

Von den insgesamt ausgewerteten 20 Zwergfledermäusen aus den Jahren 1995 bis 1999 weisen fünf Tiere Bleigehalte oberhalb der zu erwartenden mittleren Belastung von $10 \mu \mathrm{g} / \mathrm{g}$ TS auf. Vier dieser Tiere stammten aus Innenstadtlagen von Hannover, Salzgitter und Bückeburg. Ein Tier (Nr. 96021) mit einem Knochenbleigehalt von $30 \mathrm{\mu g} / \mathrm{g}$ TS stammte aus einem Quartier bei Greene im Landkreis Northeim. Alle Tiere sind als adulte Exemplare einzustufen. Bei Berücksichtigung der gesamten untersuchten Stichprobe ergibt sich keine signifikante Korrelation zwischen der Unterarmlänge und dem Knochenbleigehalt $(r=0,253)$. Bei Ausklammerung der fünf hochbelasteten Tiere ist hingegen eine deutliche Beziehung zwischen den beiden Meßgrößen erkennbar $(r=0,722)$. Die untersuchten Zwergfledermäuse (ohne die hochbelasteten Tiere) zeigen mit zunehmendem Lebensalter einen logarithmischen Anstieg 
des Knochenbleigehaltes. Etwa mit Erreichen der Geschlechtsreife ist bei nicht bleiexponierten Tieren kein weiterer Anstieg des Knochenbleigehaltes mehr erkennbar (Abb. 9).

Ähnlich verhält es sich bei den untersuchten Großen Mausohren. Auch hier kommt es zu einem logarithmisch verlaufenden Anstieg des Knochenbleigehaltes mit zunehmendem Alter, der etwa mit Beginn der Geschlechtsreife beendet ist (Abb. 10). Die ausgewertete Stichprobengruppe aus den Jahren 1993 bis 1999 stammt gleichfalls aus harzfernen Bereichen. Auch in dieser Stichprobe haben von insgesamt 19 untersuchten Tieren drei Tiere auffällig hohe Knochenbleigehalte. Diese Tiere stammten alle aus dem Jahr 1993 und wurden in einem Quartier bei Greene, Landkreis Northeim, gefunden. Für den gesamten Stichprobenumfang ergibt sich keine signifikante Korrelation $(r=0,138)$, bei Ausschluß der drei hochbelasteten Tiere ergibt sich hingegen eine deutliche Korrelation $(r=0,841)$ zwischen der Unterarmlänge und dem Knochenbleigehalt.

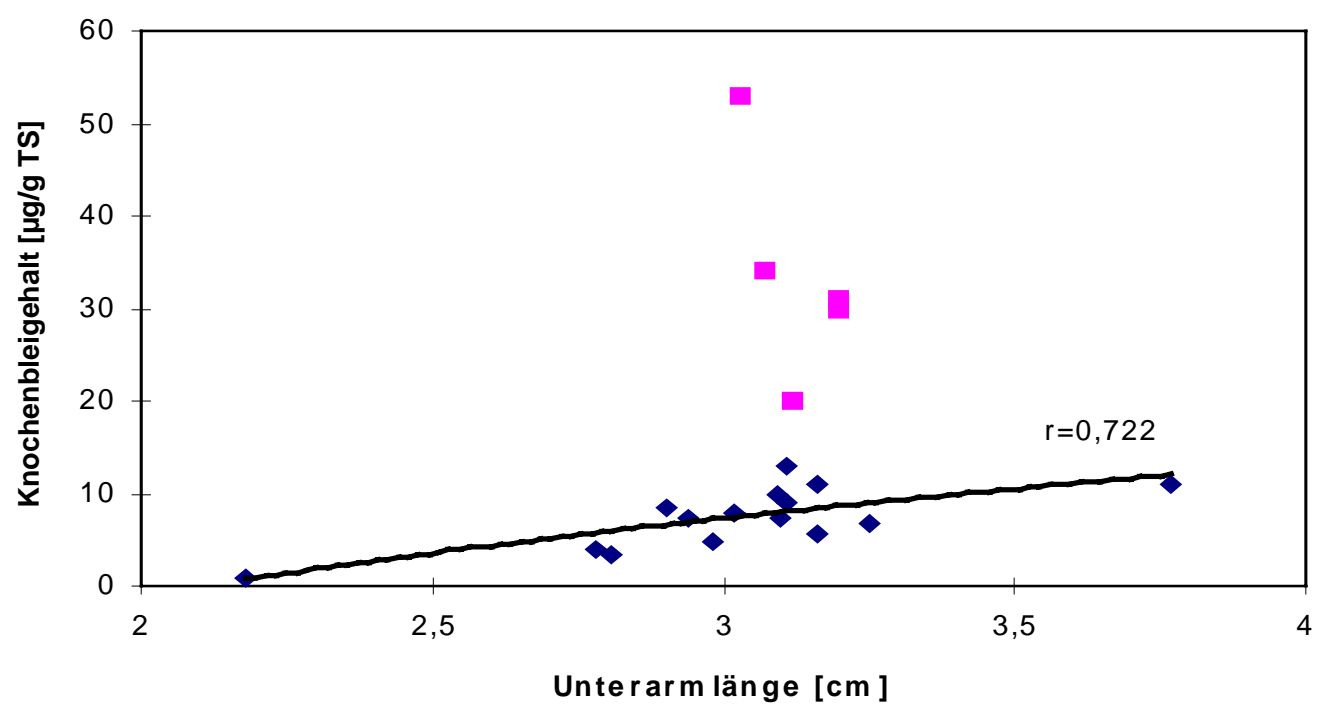

Abb. 9: Korrelation zwischen Unterarmlänge (Alter) und dem Knochenbleigehalt bei der Zwergfledermaus (P. pipistrellus). Alle Tiere stammten aus harzfernen Bereichen, Fundzeitraum 1995-1999. Die nicht gewerteten hochbelasteten Tiere $(\varpi)$ stammen mit einer Ausnahme aus Innenstadtlagen von Hannover, Salzgitter und Bückeburg. 


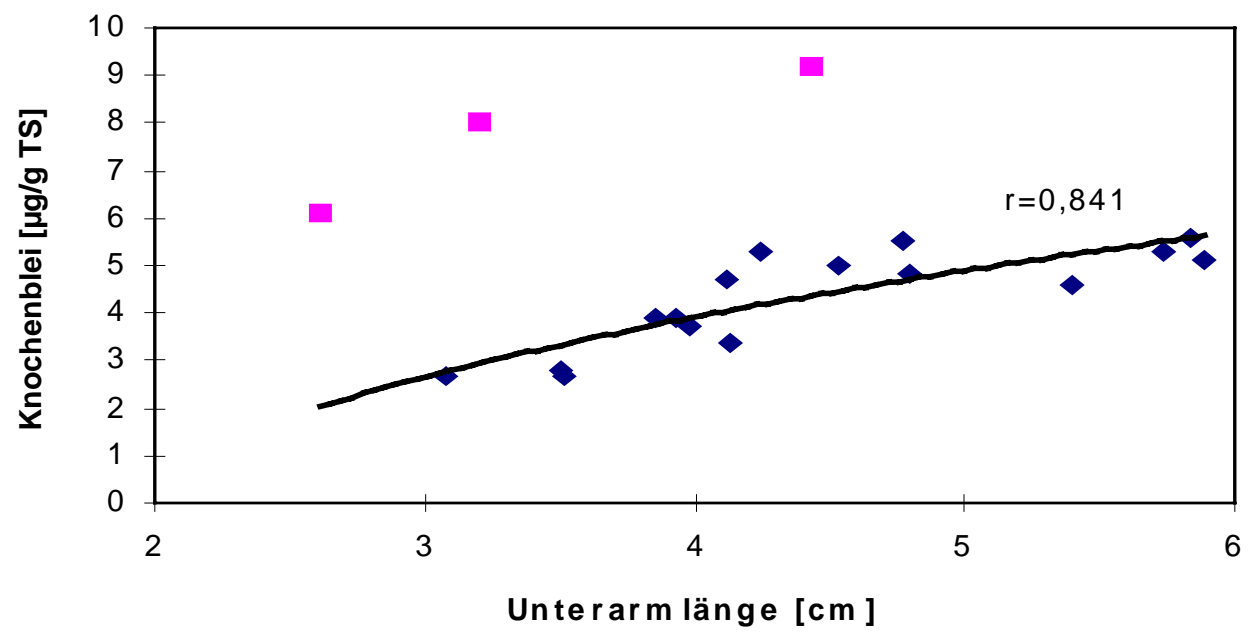

Abb. 10: Korrelation zwischen Unterarmlänge (Alter) und dem Knochenbleigehalt beim Großen Mausohr (M. myotis). Alle Tiere stammten aus harzfernen Bereichen, Fundzeitraum 1993-1999. Die nicht gewerteten hochbelasteten Tiere ( $\square$ ) stammen alle aus dem Jahr 1993 aus einem Quartier bei Greene.

\section{Geschlechtsspezifische Unterschiede:}

Ein geschlechtsspezifischer Unterschied hinsichtlich des Schadstoffgehaltes in Fledermäusen ist für Organochlorverbindungen aus verschiedenen Untersuchungen bekannt (NAGEL 1998). Hinsichtlich des Bleigehaltes liegen nur Angaben für Ganztieraufschlüsse von CLARK (1979) vor, der bei Eptesicus fuscus eine höhere Bleibelastung in Männchen als in Weibchen fand. Die vorliegenden Untersuchungen ergaben für keinen Untersuchungszeitraum und für keine Region einen signifikanten Unterschied hinsichtlich der Knochenbleibelastung zwischen Männchen und Weibchen (Tab. 14). Zur Auswertung wurden bei dieser Fragestellung nur subadulte und adulte Tiere herangezogen, um den Einfluß der niedrigeren Bleigehalte in juvenilen Tieren bei der Berechnung des Medianwertes zu vermeiden.

Tab. 14: Medianwerte [ $\mu \mathrm{g} / \mathrm{g}$ TS] der Knochenbleigehalte von subadulten und adulten männlichen und weiblichen Zwergfledermäusen ( $P$. pipistrellus) für den Betrachtungszeitraum 1996-1999 sowie Angabe der Signifikanz des Unterschiedes der Knochenbleigehalte beider Gruppen

\begin{tabular}{lccc}
\hline \hline \multicolumn{1}{c}{ Region } & Männchen & Weibchen & $\mathbf{p}$ \\
\hline Harz & $17(\mathrm{n}=35)$ & $19(\mathrm{n}=14)$ & 0,514 \\
harzfern & $9(\mathrm{n}=11)$ & $9,5(\mathrm{n}=6)$ & 0,627 \\
\hline \hline
\end{tabular}




\section{Artspezifische Unterschiede:}

Die Knochenbleigehalte der subadulten und adulten Zwergfledermäuse aus dem Fundzeitraum 1993 bis 1999 liegen im Vergleich zu den Totfunden vom Großen Mausohr aus demselben Zeitraum signifikant höher $(p=0,003)$, für den ausreißerbereinigten Stichprobenumfang sogar hochsignifikant höher $(p=0,0001)$. Der gegenwärtige mittlere Knochenbleigehalt adulter Zwergfledermäuse (ausreißerbereinigt) kann für die zweite Hälfte der neunziger Jahre etwa mit $7,5 \mu \mathrm{g} / \mathrm{g}$ TS angesetzt werden, für adulte Tiere des Großen Mausohres mit 4,4 $\mu \mathrm{g} / \mathrm{g}$ TS. Damit liegt der Knochenbleigehalt bei der Zwergfledermaus um rund $70 \%$ höher als beim Großen Mausohr (Abb. 11). Als Ausreißer wurden hierbei die hochbelasteten Tiere aus dem Quartier bei Greene, Landkreis Northeim, und aus Innenstadtlagen bezeichnet.

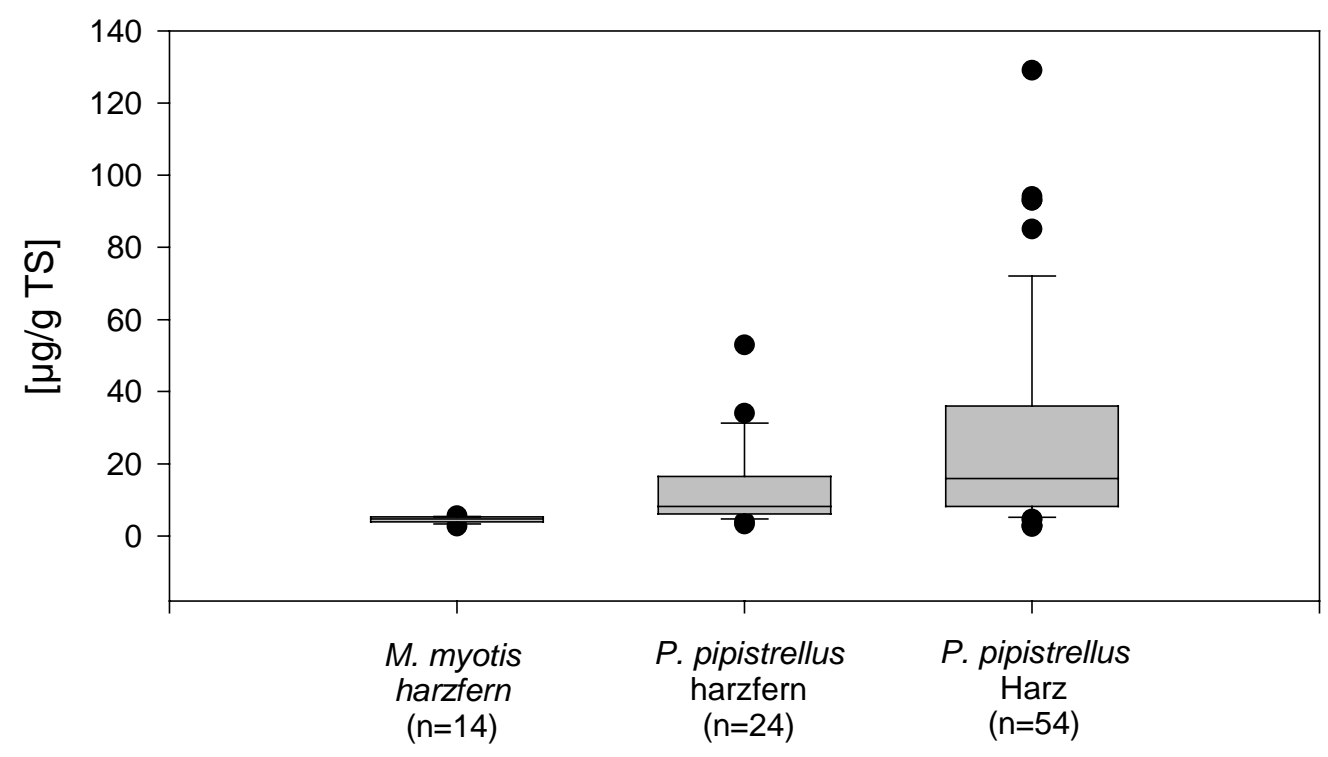

Abb. 11: Box-Plot-Diagramme der Knochenbleigehalte von subadulten und adulten Großen Mausohren (M. myotis) und Zwergfledermäusen ( $P$. pipistrellus) der Totfunde aus den Jahren 1993-1999.

\subsection{Schwermetallgehalte in Organen}

Untersuchungen von Gewebeproben verschiedener Organe wurden ausschließlich an Zwergfledermäusen durchgeführt. Untersucht wurden jeweils Nieren und Leber. Blei wurde zur Erfassung der Schadstoffinkorporation über den Luftpfad zusätzlich im Lungengewebe analysiert. Die Auflistung der Einzelmeßwerte mit ergänzenden Angaben zu Geschlecht, Alter und Herkunft finden sich im Anhang 4. 


\subsubsection{Blei}

Die Bleianreicherung in den verschiedenen untersuchten Organen der Zwergfledermaus verhält sich wie folgt:

$$
\text { Lunge }<\text { Nieren }<\text { Leber. }
$$

Analog zu den Knochenbleigehalten streuen die Werte im allgemeinen bei Tieren aus dem Bereich des Harzes deutlich stärker als bei Proben aus dem übrigen Niedersachsen (Abb. 12, Tab. 15). Die Bleigehalte in Leber und Nieren liegen bei den Totfunden aus dem Harz deutlich bis signifikant über denen von Totfunden aus dem übrigen Niedersachsen (Tab. 16). Eine erhöhte Bleianreicherung im Lungengewebe der aus dem Harz stammenden Tiere war hingegen nicht nachweisbar.

Tab. 15: Statistische Kenngrößen der Bleigehalte in Gewebeproben von Zwergfledermäusen ( $P$. pipistrellus)

Harz:

\begin{tabular}{|c|c|c|c|}
\hline & Lunge & Leber & Nieren \\
\hline Stichprobengröße & 9 & 9 & 12 \\
\hline Mittelwert [ $\mu \mathrm{g} / \mathrm{g}$ TS] & 2,4 & 6,4 & 3,3 \\
\hline Median [ $\mu \mathrm{g} / \mathrm{g} \mathrm{TS}]$ & 1,0 & 6,3 & 2,7 \\
\hline Standardabweichung $[\mu \mathrm{g} / \mathrm{g}$ TS] & 2,77 & 2,81 & 2,03 \\
\hline Schiefe & 1,702 & $-0,073$ & 1,286 \\
\hline Spannweite $[\mu \mathrm{g} / \mathrm{g}$ TS] & 8,48 & 7,2 & 6,2 \\
\hline Minimum $[\mu \mathrm{g} / \mathrm{g}$ TS] & 0,22 & 2,6 & 1,1 \\
\hline Maximum $[\mu \mathrm{g} / \mathrm{g} T S]$ & 8,7 & 9,8 & 7,3 \\
\hline
\end{tabular}

harzfern:

\begin{tabular}{|c|c|c|c|}
\hline & Lunge & Leber & Nieren \\
\hline Stichprobengröße & 7 & 9 & 10 \\
\hline Mittelwert $[\mu \mathrm{g} / \mathrm{g}$ TS] & 0,73 & 3,5 & 1,3 \\
\hline Median $[\mu \mathrm{g} / \mathrm{g}$ TS $]$ & 0,80 & 2,0 & 1,2 \\
\hline Standardabweichung $[\mu \mathrm{g} / \mathrm{g}$ TS] & 0,22 & 3,05 & 0,71 \\
\hline Schiefe & $-0,797$ & 1,428 & 1,026 \\
\hline Spannweite $[\mu \mathrm{g} / \mathrm{g}$ TS] & 0,62 & 8,90 & 2,17 \\
\hline Minimum $[\mu \mathrm{g} / \mathrm{g}$ TS] & 0,35 & 1,1 & 0,53 \\
\hline Maximum $[\mu \mathrm{g} / \mathrm{g}$ TS] & 0,97 & 10 & 2,7 \\
\hline
\end{tabular}

Niedersachsen, gesamt:

\begin{tabular}{|c|c|c|c|}
\hline & Lunge & Leber & Nieren \\
\hline Stichprobengröße & 16 & 18 & 22 \\
\hline Mittelwert $[\mu \mathrm{g} / \mathrm{g}$ TS] & 1,7 & 5,0 & 2,4 \\
\hline Median [ $\mu \mathrm{g} / \mathrm{g}$ TS] & 0,85 & 4 & 1,9 \\
\hline Standardabweichung $[\mu \mathrm{g} / \mathrm{g}$ TS] & 2,21 & 3,19 & 1,85 \\
\hline Schiefe & 2,543 & 0,397 & 1,702 \\
\hline Spannweite $[\mu \mathrm{g} / \mathrm{g}$ TS] & 8,48 & 8,90 & 6,77 \\
\hline Minimum $[\mu \mathrm{g} / \mathrm{g} \mathrm{TS}]$ & 0,22 & 1,1 & 0,53 \\
\hline Maximum $[\mu \mathrm{g} / \mathrm{g}$ TS $]$ & 8,7 & 10 & 7,3 \\
\hline
\end{tabular}



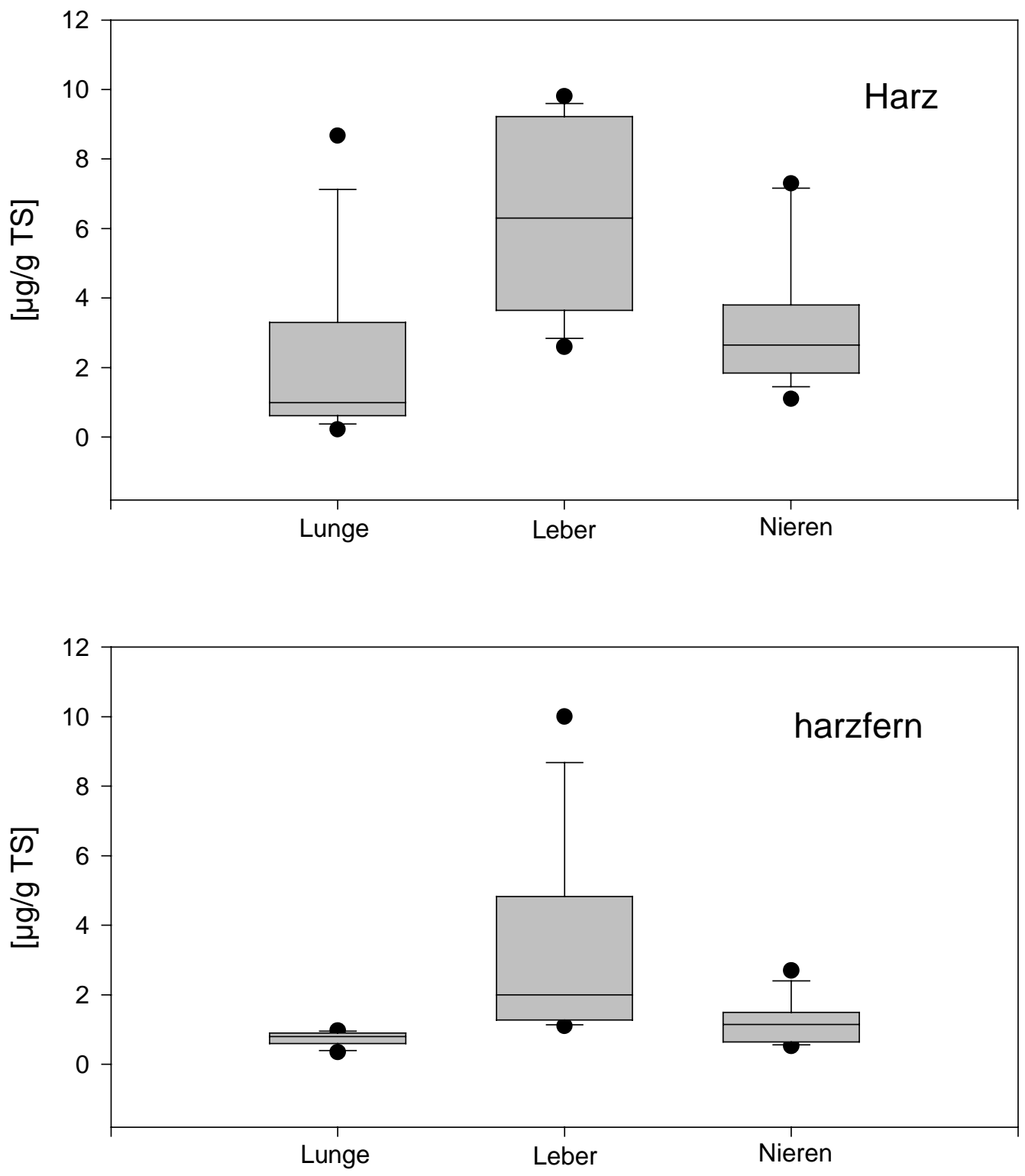

Abb. 12: Bleigehalte in Gewebeproben adulter Zwergfledermäuse ( $P$. pipistrellus) aus dem Bereich des Harzes sowie dem übrigen Niedersachsen. 
Tab. 16: Ergebnisse der U-Testprüfung auf Unterscheidung der Mediane der Bleigehalte in Gewebeproben zwischen Totfunden aus dem Harz und Totfunden aus dem übrigen Niedersachsen für die drei untersuchten Gewebe

\begin{tabular}{lcccccc}
\hline \hline & \multicolumn{2}{c}{ Lunge } & \multicolumn{2}{c}{ Leber } & \multicolumn{2}{c}{ Nieren } \\
& Harz & harzfern & Harz & harzfern & Harz & harzfern \\
\hline Stichprobengröße & 9 & 7 & 9 & 9 & 12 & 10 \\
Median [ $\mu \mathrm{g} / \mathrm{g}$ TS] & 1,0 & 0,80 & 6,3 & 2,0 & 2,7 & 1,2 \\
\hline $\mathrm{p}$ & & 0,22 & \multicolumn{2}{c}{0,057} & \multicolumn{2}{c}{0,002} \\
\hline \hline
\end{tabular}

\subsubsection{Cadmium}

Die Cadmiumanreicherung in den untersuchten Organen der Zwergfledermaus verhält sich analog der Bleiverteilung. Es gilt:

$$
\text { Nieren }<\text { Leber }
$$

Ein signifikanter Unterschied der Cadmiumgehalte im Gewebe zwischen Totfunden aus dem Bereich des Harzes und aus dem übrigen Niedersachsen war weder im Nierengewebe noch im Lebergewebe nachweisbar ( $p=0,692$ bzw. 0,821). Die Tiere aus dem Harz zeigen jedoch wiederum stärkere Streuungen der Meßwerte im Vergleich zu den im übrigen Niedersachsen aufgefundenen Tieren (Abb. 13). Der Medianwert der Cadmiumgehalte im Nieren- und im Lebergewebe aller untersuchten Tiere beträgt $0,26 \mu \mathrm{g} / \mathrm{g}$ TS bzw. 0,61 $\mu \mathrm{g} / \mathrm{g}$ TS (Tab. 17).

Tab. 17: Statistische Kenngrößen der Cadmiumgehalte in Gewebeproben von Zwergfledermäusen (P. pipistrellus)

Harz:

\begin{tabular}{lcl}
\hline \hline & Leber & Nieren \\
\hline Stichprobengröße & 12 & 10 \\
Mittelwert [ $\mu \mathrm{g} / \mathrm{g}$ TS] & 2,7 & 1,7 \\
Median $[\mu \mathrm{g} / \mathrm{g}$ TS] & 0,27 & 0,25 \\
Standardabweichung $[\mu \mathrm{g} / \mathrm{g}$ TS] & 4,33 & 2,45 \\
Schiefe & 1,978 & 1,413 \\
Spannweite $[\mu \mathrm{g} / \mathrm{g}$ TS] & 14,0 & 6,45 \\
Minimum $[\mu \mathrm{g} / \mathrm{g}$ T] & 0,0045 & 0,049 \\
Maximum $[\mu \mathrm{g} / \mathrm{g}$ TS] & 14 & 6,5 \\
\hline \hline
\end{tabular}


noch Tabelle 17:

harzfern:

\begin{tabular}{|c|c|c|}
\hline & Leber & Nieren \\
\hline Stichprobengröße & 10 & 10 \\
\hline Mittelwert [ $\mu \mathrm{g} / \mathrm{g}$ TS] & 1,65 & 1,14 \\
\hline Median [ $\mu \mathrm{g} / \mathrm{g}$ TS] & 0,76 & 0,50 \\
\hline Standardabweichung [ $\mathrm{\mu g} / \mathrm{g}$ TS] & 2,52 & 1,89 \\
\hline Schiefe & 2,563 & 2,727 \\
\hline Spannweite $[\mu \mathrm{g} / \mathrm{g}$ TS] & 8,34 & 6,25 \\
\hline Minimum $[\mu \mathrm{g} / \mathrm{g} \mathrm{TS}]$ & 0,059 & 0,05 \\
\hline Maximum $[\mu \mathrm{g} / \mathrm{g}$ TS] & 8,4 & 6,3 \\
\hline
\end{tabular}

Niedersachsen, gesamt:

\begin{tabular}{|c|c|c|}
\hline & Leber & Nieren \\
\hline Stichprobengröße & 22 & 20 \\
\hline Mittelwert [ $\mu \mathrm{g} / \mathrm{g}$ TS] & 2,20 & 1,40 \\
\hline Median [ $\mu \mathrm{g} / \mathrm{g}$ TS] & 0,61 & 0,26 \\
\hline 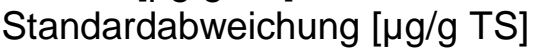 & 3,58 & 2,15 \\
\hline Schiefe & 2,236 & 1,769 \\
\hline Spannweite $[\mu \mathrm{g} / \mathrm{g}$ TS] & 14,0 & 6,45 \\
\hline Minimum $[\mu \mathrm{g} / \mathrm{g} \mathrm{TS}]$ & 0,0045 & 0,049 \\
\hline Maximum $[\mu \mathrm{g} / \mathrm{g}$ TS] & 14 & 6,5 \\
\hline
\end{tabular}

\subsubsection{Chrom}

Die Chromanreicherung in den untersuchten Organen der Zwergfledermaus verhält sich analog der anderen analytischen Schwermetalle. Es gilt:

$$
\text { Nieren < Leber }
$$

Ein statistisch signifikanter Unterschied der Chromgehalte im Gewebe zwischen Totfunden aus dem Bereich des Harzes und aus dem übrigen Niedersachsen war weder im Nierengewebe noch im Lebergewebe nachweisbar $(p=0,464$ bzw. 0,086$)$. Die Streuung der Meßwerte war für beide Gruppen annähernd identisch und im Vergleich zu Blei und Cadmium deutlich geringer (Abb. 14). Die Medianwerte der Chromgehalte im Nieren- und im Lebergewebe aller untersuchten Tiere betragen $0,049 \mu \mathrm{g} / \mathrm{g}$ TS bzw. 0,080 $\mu \mathrm{g} / \mathrm{g}$ TS (Tab. 18). 

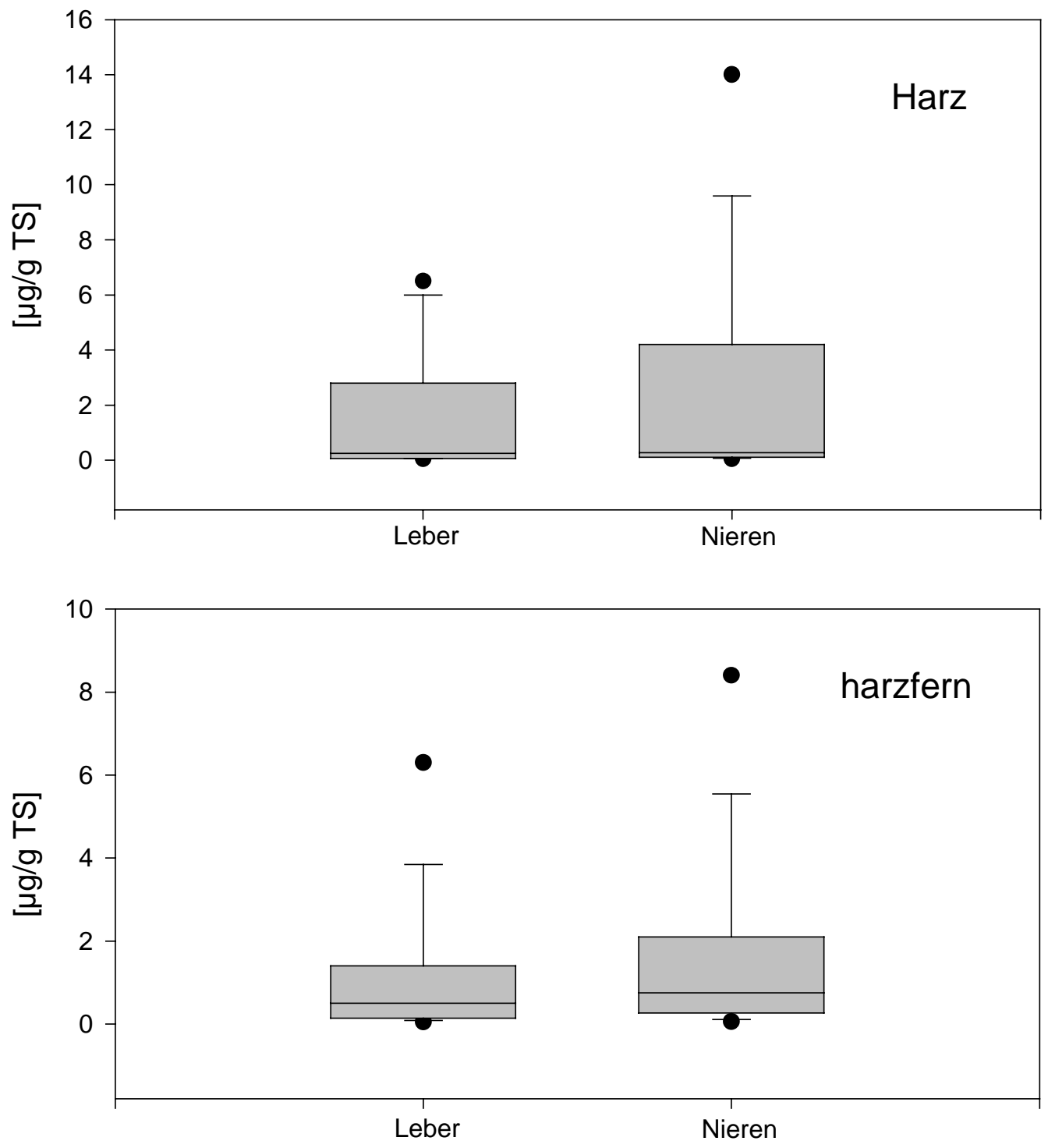

Abb. 13: Cadmiumgehalte in Gewebeproben adulter Zwergfledermäuse ( $P$. pipistrellus) aus dem Bereich des Harzes sowie dem übrigen Niedersachsen. 
Tab. 18: Statistische Kenngrößen der Chromgehalte in Gewebeproben von Zwergfledermäusen (P. pipistrellus)

Harz:

\begin{tabular}{lll}
\hline \hline & Leber & Nieren \\
\hline Stichprobengröße & 12 & 10 \\
Mittelwert [ $\mu \mathrm{g} / \mathrm{g}$ TS] & 0,21 & 0,11 \\
Median $[\mu \mathrm{g} / \mathrm{g}$ TS] & 0,054 & 0,034 \\
Standardabweichung $[\mu \mathrm{g} / \mathrm{g}$ TS] & 0,47 & 0,18 \\
Schiefe & 3,395 & 2,821 \\
Spannweite $[\mu \mathrm{g} / \mathrm{g}$ TS] & 1,68 & 0,58 \\
Minimum $[\mu \mathrm{g} / \mathrm{g}$ TS] & 0,025 & 0,02 \\
Maximum $[\mu \mathrm{g} / \mathrm{g}$ TS] & 1,7 & 0,6 \\
\hline \hline
\end{tabular}

harzfern:

\begin{tabular}{lcc}
\hline \hline & Leber & Nieren \\
\hline Stichprobengröße & 10 & 11 \\
Mittelwert $[\mu \mathrm{g} / \mathrm{g}$ TS] & 0,30 & 0,23 \\
Median $[\mu \mathrm{g} / \mathrm{g}$ TS] & 0,17 & 0,069 \\
Standardabweichung $[\mu \mathrm{g} / \mathrm{g}$ TS] & 0,42 & 0,53 \\
Schiefe & 2,415 & 3,276 \\
Spannweite $[\mu \mathrm{g} / \mathrm{g}$ TS] & 1,36 & 1,78 \\
Minimum $[\mu \mathrm{g} / \mathrm{g}$ TS] & 0,040 & 0,025 \\
Maximum $[\mu \mathrm{g} / \mathrm{g}$ TS] & 1,4 & 1,8 \\
\hline \hline
\end{tabular}

Niedersachsen, gesamt:

\begin{tabular}{lcc}
\hline \hline & Leber & Nieren \\
\hline Stichprobengröße & 22 & 21 \\
Mittelwert [ $\mu \mathrm{g} / \mathrm{g}$ TS] & 0,25 & 0,17 \\
Median $[\mu \mathrm{g} / \mathrm{g}$ TS] & 0,080 & 0,049 \\
Standardabweichung $[\mu \mathrm{g} / \mathrm{g}$ TS] & 0,44 & 0,40 \\
Schiefe & 2,74 & 3,96 \\
Spannweite $[\mu \mathrm{g} / \mathrm{g}$ TS] & 1,68 & 1,78 \\
Minimum $[\mu \mathrm{g} / \mathrm{g}$ TS] & 0,025 & 0,02 \\
Maximum [ $\mu \mathrm{g} / \mathrm{g}$ TS] & 1,7 & 1,8 \\
\hline \hline
\end{tabular}



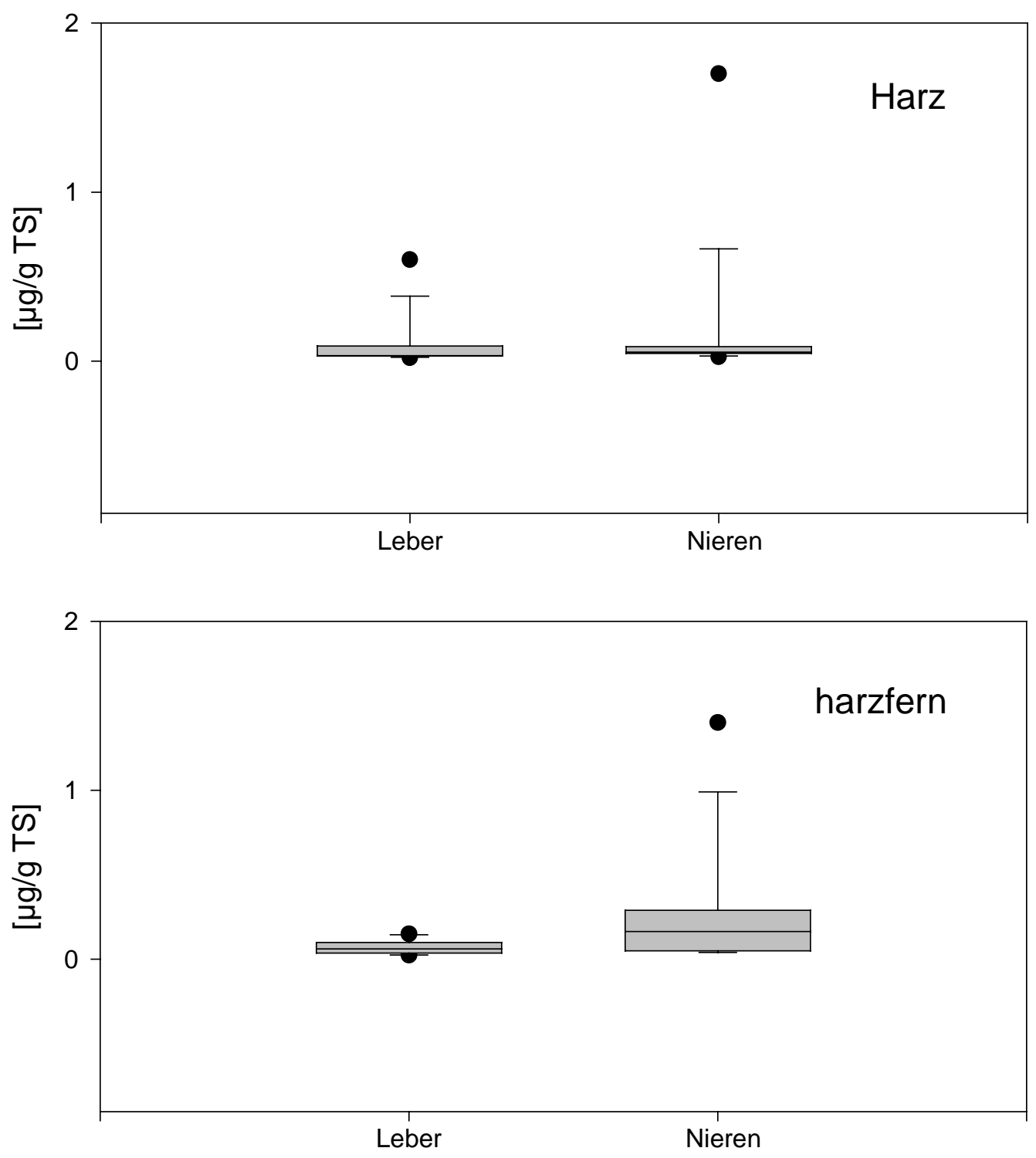

Abb. 14: Chromgehalte in Gewebeproben adulter Zwergfledermäuse ( $P$. pipistrellus) aus dem Bereich des Harzes sowie dem übrigen Niedersachsen. 


\subsection{Schwermetallgehalte in Haaren}

Untersuchungen an Haarproben wurden aufgrund des für die übrigen Arten zur Verfügung stehenden geringen Stichprobenumfanges ausschließlich an Zwergfledermäusen durchgeführt. Es erfolgte primär die Beprobung des Rückenfelles. Nur bei juvenilen Tieren mit Haarlängen unter $3 \mathrm{~mm}$ oder schlecht erhaltenen Tieren wurde ergänzend das Bauchfell mit herangezogen. Die Auflistung der Einzelmeßwerte mit ergänzenden Angaben zu Geschlecht, Alter und Herkunft finden sich im Anhang 5. Die Zusammenstellung der statistischen Kenngrößen sowie die graphische Darstellung der Verteilung für alle gemessenen Elemente befindet sich am Ende dieses Kapitels (Tab. 32a-c, Abb. 17) . Alle nachstehenden Elementkonzentrationen beziehen sich auf Haarproben, die bei einer relativen Luftfeuchte von $50 \%$ äquilibriert wurden.

\subsubsection{Blei}

\section{Entwicklung der Bleigehalte im Haar im Zeitraum 1987 bis 1999:}

Analog den Ergebnissen der Knochenbleibestimmungen nahm im Zeitraum von 1987 bis 1999 der Haarbleigehalt aller Tiere deutlich ab. Bei den Tieren aus dem harzfernen Bereich ging der Median der Haarbleigehalte von rund $56 \mu \mathrm{g} / \mathrm{g}$ zu Beginn des Betrachtungszeitraumes (1987) auf rund $23 \mu \mathrm{g} / \mathrm{g}$ gegen Ende des Betrachtungszeitraumes (1999), d.h., um rund 55 \% zurück (Abb. 15, Tab. 19).

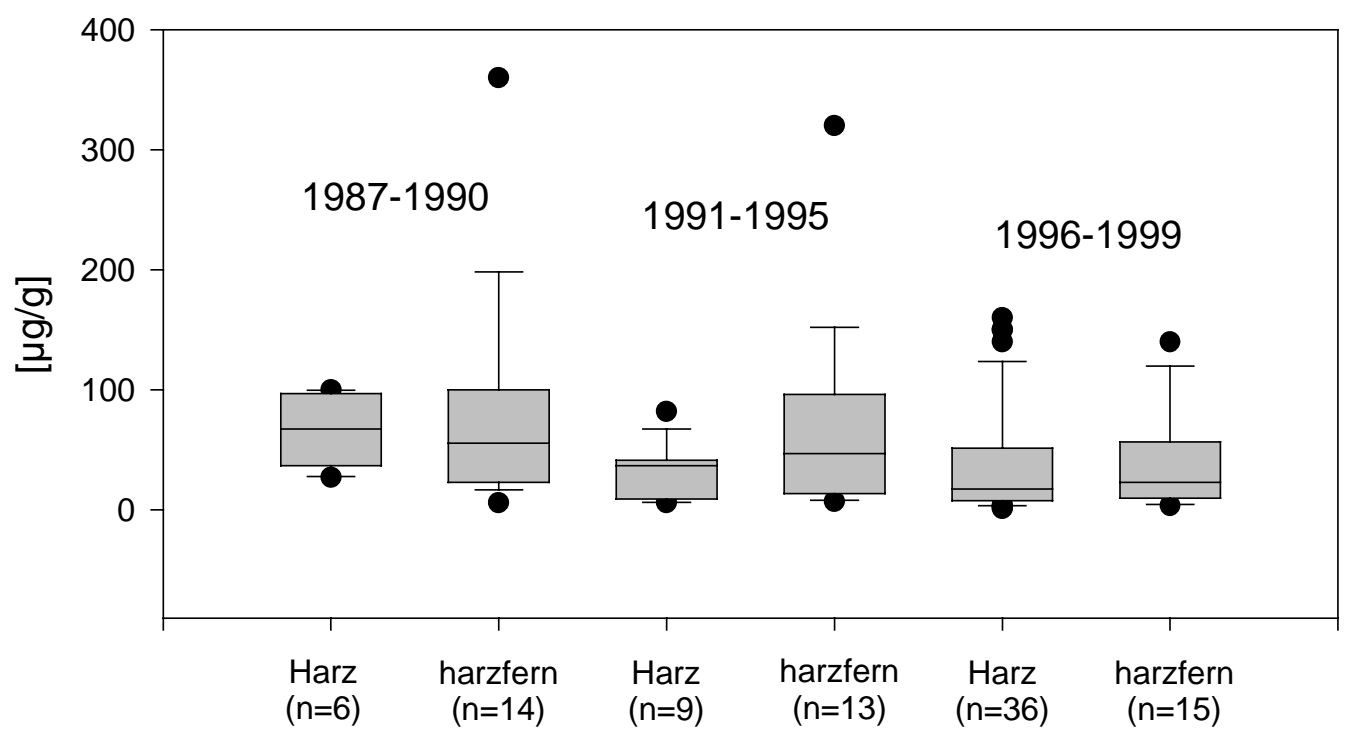

Abb. 15: Box-Plot-Diagramme der ermittelten Haarbleigehalte der untersuchten Zwergfledermäuse ( $P$. pipistrellus) aus dem Bereich des Harzes und aus harzfernen Bereichen im Zeitraum 1987-1999. 
Tab. 19: Vergleich der statistischen Kenngrößen der Haarbleigehalte in Zwergfledermäusen ( $P$. pipistrellus) aus dem Zeitraum 1987 bis 1999 aus dem Bereich des Harzes sowie aus harzfernen Bereichen

Harz:

\begin{tabular}{lcccc}
\hline \hline & $\mathbf{1 9 8 7 - 1 9 9 0}$ & $\mathbf{1 9 9 1 - 1 9 9 5}$ & $\mathbf{1 9 9 6 - 1 9 9 9}$ & $\mathbf{1 9 8 7 - 1 9 9 9}$ \\
\hline Stichprobenumfang & 6 & 9 & 36 & 51 \\
Mittelwert $[\mu \mathrm{g} / \mathrm{g}]$ & 66 & 33 & 37 & 39 \\
Median $[\mu \mathrm{g} / \mathrm{g}]$ & 68 & 37 & 18 & 28 \\
Standardabweichung $[\mu \mathrm{g} / \mathrm{g}]$ & 31,5 & 24,0 & 43,6 & 40,3 \\
Schiefe & $-0,12$ & 0,83 & 1,78 & 1,53 \\
Spannweite $[\mu \mathrm{g} / \mathrm{g}]$ & 73 & 76 & 158,8 & 158,8 \\
Minimum $[\mu \mathrm{g} / \mathrm{g}]$ & 27 & 6 & 1,2 & 1,2 \\
Maximum $[\mu \mathrm{g} / \mathrm{g}]$ & 100 & 82 & 160 & 160 \\
\hline \hline
\end{tabular}

harzfern:

\begin{tabular}{lcccc}
\hline \hline & $\mathbf{1 9 8 7 - 1 9 9 0}$ & $\mathbf{1 9 9 1 - 1 9 9 5}$ & $\mathbf{1 9 9 6 - 1 9 9 9}$ & $\mathbf{1 9 8 7 - 1 9 9 9}$ \\
\hline Stichprobenumfang & 14 & 13 & 15 & 42 \\
Mittelwert $[\mu \mathrm{g} / \mathrm{g}]$ & 89 & 72 & 39 & 66 \\
Median $[\mu \mathrm{g} / \mathrm{g}]$ & 56 & 47 & 23 & 40 \\
Standardabweichung $[\mu \mathrm{g} / \mathrm{g}]$ & 95,1 & 83,1 & 42,3 & 77,1 \\
Schiefe & 2,02 & 2,48 & 1,54 & 2,38 \\
Spannweite $[\mu \mathrm{g} / \mathrm{g}]$ & 354,1 & 313,2 & 136,6 & 356,6 \\
Minimum $[\mu \mathrm{g} / \mathrm{g}]$ & 5,9 & 6,8 & 3,4 & 3,4 \\
Maximum $[\mu \mathrm{g} / \mathrm{g}]$ & 360 & 320 & 140 & 360 \\
\hline \hline
\end{tabular}

Signifikante Unterschiede hinsichtlich der Haarbleigehalte zwischen Totfunden aus dem Bereich des Harzes und Totfunden aus dem übrigen Niedersachsen waren, anders als bei den Knochenbleigehalten, statistisch nicht nachweisbar (Tab. 20). Bei genauer Betrachtung der Fundorte der Jahre 1996-1999 kann festgestellt werden, daß Tiere aus innerstädtischen bzw. stadtnahen Bereichen (Hannover, Salzgitter) im Vergleich zu Totfunden in ländlichen Regionen etwa doppelt so hohe Haarbleigehalte aufweisen (Anhang 6). Besonders auffällig sind die hohen Haarbleigehalte bei den Tieren aus dem Bereich Greene-Kreiensen, Landkreis Northeim. Die beiden aus diesem Bereich stammenden Zwergfledermäuse (Tier Nr. 96016 und 96921) weisen mit $140 \mu \mathrm{g} / \mathrm{g}$ TS bzw. $120 \mu \mathrm{g} / \mathrm{g}$ TS die höchsten Haarbleigehalte im Betrachtungszeitraum 1996-1999 auf. Das Tier Nr. 96021 wies bei der Knochenbleibestimmung im selben Betrachtungszeitraum gleichfalls den höchsten Bleigehalt auf. Auch die untersuchten Großen Mausohren aus dieser Region zeigen im Vergleich zu einer Mausohrwochenstube im Stadtgebiet von Northeim deutlich erhöhte Knochenbleigehalte. Für das Tier 96016 liegen keine Meßwerte zum Knochenbleigehalt vor. 
Tab. 20: Medianwerte $[\mu \mathrm{g} / \mathrm{g}]$ der Bleigehalte im Haar von Zwergfledermäusen (P. pipistrellus) im Zeitraum 1987 bis 1999 und Ergebnisse der Signifikanzprüfung (U-Test nach MANN-WHITNEY)

\begin{tabular}{ccccc}
\hline \hline $\begin{array}{c}\text { Betrachtungs- } \\
\text { zeitraum }\end{array}$ & Alle Tiere & Tierfunde Harz & $\begin{array}{c}\text { harzferne Tier- } \\
\text { funde }\end{array}$ & $\begin{array}{c}\text { p } \\
\text { Harz/harzfern }\end{array}$ \\
\hline $1987-1990$ & $57(n=20)$ & $68(n=6)$ & $56(n=14)$ & 0,733 \\
$1991-1995$ & $40(n=22)$ & $37(n=9)$ & $47(n=13)$ & 0,133 \\
$1996-1999$ & $19(n=51)$ & $18(n=36)$ & $23(n=15)$ & 0,577 \\
\hline $1987-1999$ & $31(n=93)$ & $28(n=51)$ & $40(n=42)$ & 0,075 \\
\hline \hline
\end{tabular}

\section{Abhängigkeit vom Alter:}

Zur Beschreibung einer möglichen Altersabhängigkeit des Haarbleigehaltes wurde dieser mit der Unterarmlänge korreliert (Abb. 16). Insgesamt wurden 51 Tiere aus dem Betrachtungszeitraum 1996 bis 1999 ausgewertet. Aufgrund der starken Schwankungen im Haarbleigehalt der einzelnen Tiere war eine Altersabhängigkeit der beiden betrachteten Kollektive aus dem Harz bzw. aus harzfernen Bereichen statistisch nicht nachweisbar $(r=<<0,5)$. Besonders bei den Tieren aus dem Harz fällt jedoch in der Gruppe der adulten Tiere ein deutlich größerer Anteil an Tieren auf, deren Haarbleigehalte deutlich oberhalb des Medianwertes für das Gesamtkollektiv (juvenile, subadulte und adulte) liegen. Bei der Berechnung der Medianwerte für die Gruppe der subadulten und adulten Tiere zeigt sich dieser Unterschied noch wesentlich deutlicher. Der Median des Haarbleigehaltes der subadulten Tiere aus dem Harz $(n=14)$ beträgt $15 \mu \mathrm{g} / \mathrm{g}$, wohingegen der Median der adulten Tiere $(\mathrm{n}=23) 28 \mu \mathrm{g} / \mathrm{g}$ beträgt und damit nahezu doppelt so hoch liegt. Für die Gruppe der harzfernen Tiere zeigte sich hingegen kein Unterschied in den Medianwerten der subadulten $(n=9)$ und adulten $(\mathrm{n}=8)$ Tiere. Die berechneten Medianwerte betragen 26 bzw. $24 \mu \mathrm{g} / \mathrm{g}$.

\section{Abhängigkeit vom Geschlecht:}

Für den Betrachtungszeitraum 1996-1999 konnte bei den Zwergfledermäusen für den Haarbleigehalt bei den insgesamt untersuchten 49 subadulten und adulten Tieren für die Weibchen ein geringfügig niedrigerer Medianwert des Haarbleigehaltes ermittelt werden. Juvenile Tiere wurden aufgrund der zu erwartenden niedrigeren Bleibelastung hierbei nicht mit berücksichtigt. Die statistische Signifikanzprüfung ergab keinen gesicherten signifikanten geschlechtsspezifischen Unterschied (Tab. 21). 


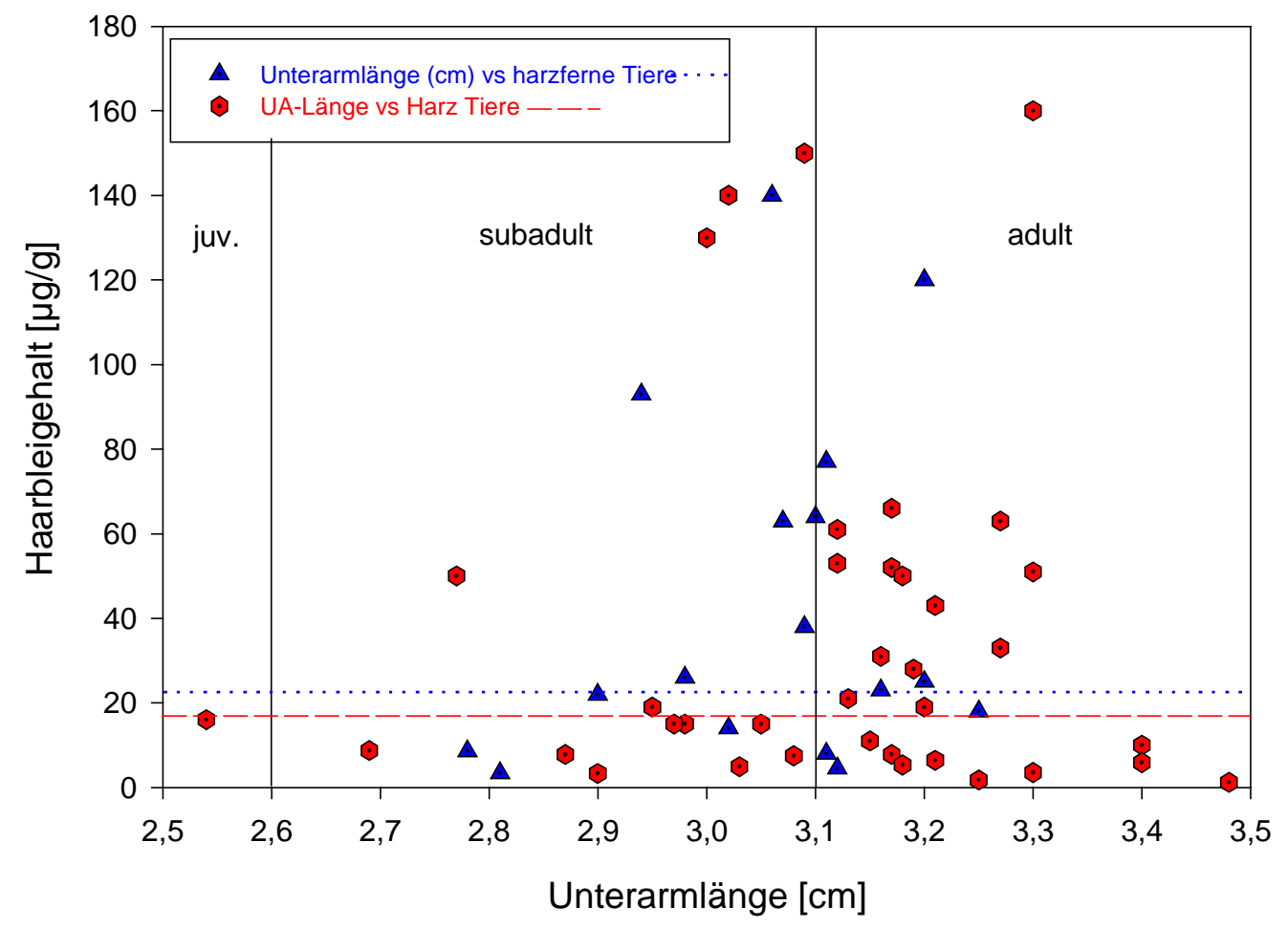

Abb. 16: Verteilung der Haarbleigehalte in Abhängigkeit der Unterarmlänge als relatives Maß für das Alter eines Tieres. Datengrundlage sind die Zwergfledermäuse (P. pipistrellus) aus dem Betrachtungszeitraum 1996-1999. Die horizontalen unterbrochenen Linien im Diagramm markieren den Medianwert des jeweiligen Datensatzes. Die senkrechten Linien trennen die jeweilige Altersklassen. Hohe Haarbleigehalte fanden sich überwiegend bei den adulten Tieren (Unterarmlänge $>3,1 \mathrm{~cm}$ ).

Tab. 21: Medianwerte $[\mu \mathrm{g} / \mathrm{g}]$ der Haarbleigehalte von männlichen und weiblichen subadulten und adulten Zwergfledermäusen ( $P$. pipistrellus) aus dem Betrachtungszeitraum 1996-1999

\begin{tabular}{llll}
\hline \hline \multicolumn{1}{c}{ Region } & Männchen & Weibchen & p \\
\hline Harz & $31(n=23)$ & $8,7(n=9)$ & 0,277 \\
harzfern & $26(n=9)$ & $22(n=4)$ & 1,000 \\
\hline Alle Tiere & $28(n=31)$ & $15(n=13)$ & 0,375 \\
\hline \hline
\end{tabular}




\subsubsection{Cadmium}

Entwicklung der Cadmiumgehalte im Haar im Zeitraum 1987 bis 1999:

Die Entwicklung der Cadmiumgehalte ergab einen abnehmenden Trend über den gesamten Betrachtungszeitraum von 1987 bis 1999. Die Abnahme der Cadmiumgehalte im Haar betrug dabei sowohl für die aus dem Harz stammenden Tiere als auch für die aus dem übrigen Niedersachsen stammenden Tiere zwischen $40 \%$ und $50 \%$. (Tab. 22, vgl. Abb. 17).

Tab. 22: Medianwerte der Cadmiumgehalte im Haar bei Zwergfledermäusen ( $P$. pipistrellus) im Zeitraum 1987 bis 1999

\begin{tabular}{ccccc}
\hline \hline $\begin{array}{c}\text { Betrachtungs- } \\
\text { zeitraum }\end{array}$ & Alle Tiere & Tierfunde Harz & $\begin{array}{c}\text { harzferne Tier- } \\
\text { funde }\end{array}$ & $\begin{array}{c}\text { p } \\
\text { Harz/harzfern }\end{array}$ \\
\hline $1987-1990$ & $0,45(n=20)$ & $0,52(n=6)$ & $0,42(n=14)$ & 0,364 \\
$1991-1995$ & $0,39(n=22)$ & $0,28(n=9)$ & $0,42(n=13)$ & 0,217 \\
$1996-1999$ & $0,25(n=50)$ & $0,16(n=35)$ & $0,31(n=15)$ & 0,037 \\
\hline $1987-1999$ & $0,35(n=92)$ & $0,23(n=50)$ & $0,41(n=42)$ & 0,012 \\
\hline \hline
\end{tabular}

\section{Abhängigkeit vom Alter:}

Die Korrelation mit der Unterarmlänge als relatives Maß für das Alter eines Tieres mit dem Cadmiumgehalt ergab keinen signifikanten Zusammenhang $(r<0,5)$. Dies gilt sowohl für das Gesamtkollektiv als auch bei ausschließlicher Betrachtung der Tiere aus dem Harz bzw. aus den harzfernen Bereichen.

\section{Abhängigkeit vom Geschlecht:}

Wie beim Blei erfolgte aufgrund des erkennbaren abnehmenden langjährigen Trends der Cadmiumgehalte im Haar die Prüfung auf geschlechtsspezifische Unterschiede nur für den Betrachtungszeitraum 1996-1999, da hier eine ausreichend große Stichprobe zur Verfügung stand. Juvenile Tiere wurden in den Vergleich nicht mit einbezogen. Bei dem Gesamtkollektiv der untersuchten Zwergfledermäuse konnte für den Cadmiumgehalt im Haar ein statistischer geschlechtsspezifischer Unterschied festgestellt werden ( $p=0,05$ für alle Tiere). Dies galt jedoch nicht bei getrennter Betrachtung der aus dem Harz und aus dem übrigen Niedersachsen stammenden Tiere (Tab. 23). 
Tab. 23: Medianwerte $[\mu \mathrm{g} / \mathrm{g}]$ der Cadmiumgehalte im Haar von männlichen und weiblichen subadulten und adulten Zwergfledermäusen ( $P$. pipistrellus) aus dem Betrachtungszeitraum 1996-1999

\begin{tabular}{lclc}
\hline \hline \multicolumn{1}{c}{ Region } & Männchen & Weibchen & $\mathbf{p}$ \\
\hline Harz & $0,35(n=22)$ & $0,12(n=9)$ & 0,060 \\
harzfern & $0,40(n=9)$ & $0,25(n=4)$ & 0,355 \\
\hline Alle Tiere & $0,37(n=31)$ & $0,16(n=13)$ & 0,047 \\
\hline \hline
\end{tabular}

\subsubsection{Chrom}

\section{Entwicklung der Chromgehalte im Haar im Zeitraum 1987 bis 1999:}

Erkennbare Unterschiede im Chromgehalt der Haare von Tieren aus dem Harz und aus harzfernen Bereichen lagen nicht vor. Für alle untersuchten Tiere konnte zudem kein erkennbarer abnehmender oder zunehmender Trend der Chromgehalte über den gesamten Betrachtungszeitraum festgestellt werden (Tab. 24).

Tab. 24: Medianwerte der Chromgehalte im Haar bei Zwergfledermäusen (P. pipistrellus) im Zeitraum 1987 bis 1999

\begin{tabular}{ccccc}
\hline \hline $\begin{array}{c}\text { Betrachtungs- } \\
\text { zeitraum }\end{array}$ & Alle Tiere & Tierfunde Harz & $\begin{array}{c}\text { harzferne Tier- } \\
\text { funde }\end{array}$ & $\begin{array}{c}\mathbf{p} \\
\text { Harz/harzfern }\end{array}$ \\
\hline $1987-1990$ & $2,1(\mathrm{n}=20)$ & $2,4(\mathrm{n}=6)$ & $1,9(\mathrm{n}=14)$ & 0,216 \\
$1991-1995$ & $2,3(\mathrm{n}=22)$ & $1,7(\mathrm{n}=9)$ & $2,8(\mathrm{n}=13)$ & 0,443 \\
$1996-1999$ & $2,1(\mathrm{n}=50)$ & $2,0(\mathrm{n}=35)$ & $2,7(\mathrm{n}=15)$ & 0,427 \\
\hline $1987-1999$ & $2,1(\mathrm{n}=92)$ & $2,0(\mathrm{n}=50)$ & $2,5(\mathrm{n}=42)$ & 0,562 \\
\hline \hline
\end{tabular}

\section{Abhängigkeit vom Alter:}

Die Korrelation mit der Unterarmlänge als relatives Maß für das Alter eines Tieres mit dem Chromgehalt ergab, wie bereits für Cadmium festgestellt, keinen signifikanten Zusammenhang $(r<0,5)$. Dies gilt sowohl für das Gesamtkollektiv als auch bei ausschließlicher Betrachtung der Tiere aus dem Harz bzw. aus den harzfernen Bereichen.

\section{Abhängigkeit vom Geschlecht:}

Da der Chromgehalt während des gesamten Betrachtungszeitraumes 1987-1999 keinen signifikanten Änderungen unterlag, wurde zur Prüfung auf geschlechtsspezifischen Unterschiede im Chromgehalt der Haare das gesamte Probenkollektiv von insgesamt 82 subadulten und adulten Tieren einbezogen. Juvenile Tiere wurden 
hierbei nicht berücksichtigt. Bei den untersuchten Zwergfledermäusen konnte für den Chromgehalt im Haar kein signifikanter geschlechtsspezifischer Unterschied festgestellt werden. Dies galt sowohl für die aus dem Harz stammenden Tiere als auch für die Tiere aus dem übrigen Niedersachsen (Tab. 25).

Tab. 25: Medianwerte $[\mu \mathrm{g} / \mathrm{g}]$ der Chromgehalte im Haar von männlichen und weiblichen subadulten und adulten Zwergfledermäusen ( $P$. pipistrellus) aus dem Betrachtungszeitraum 1987-1999

\begin{tabular}{lccc}
\hline \hline \multicolumn{1}{c}{ Region } & Männchen & Weibchen & $\mathbf{p}$ \\
\hline Harz & $2,0(n=29)$ & $2,0(n=15)$ & 0,595 \\
harzfern & $2,5(n=26)$ & $2,6(n=12)$ & 0,649 \\
\hline Alle Tiere & $2,1(n=55)$ & $2,1(n=27)$ & 0,661 \\
\hline \hline
\end{tabular}

\subsubsection{Kupfer}

\section{Entwicklung der Kupfergehalte im Haar im Zeitraum 1987 bis 1999:}

Die Entwicklung der Kupfergehalte über den gesamten Betrachtungszeitraum ergab keinen Hinweis auf einen signifikanten abnehmenden oder zunehmenden Trend. Im Betrachtungszeitraum 1991-1995 lagen die Medianwerte für beide Fallgruppen jedoch deutlich über denen der beiden anderen Betrachtungszeiträume. Für den Betrachtungszeitraum 1996-1999 lagen die Kupfergehalte der Tiere aus harzfernen Bereichen zudem signifikant über denen aus dem Bereich des Harzes (Tab. 26).

Tab. 26: Medianwerte der Kupfergehalte im Haar bei Zwergfledermäusen (P. pipistrellus) im Zeitraum 1987 bis 1999

\begin{tabular}{ccccc}
\hline \hline $\begin{array}{c}\text { Betrachtungs- } \\
\text { zeitraum }\end{array}$ & Alle Tiere & Tierfunde Harz & $\begin{array}{c}\text { harzferne Tier- } \\
\text { funde }\end{array}$ & $\begin{array}{c}\text { p } \\
\text { Harz/harzfern }\end{array}$ \\
\hline $1987-1990$ & $18(n=20)$ & $28(n=6)$ & $17(n=14)$ & 0,322 \\
$1991-1995$ & $28(n=22)$ & $32(n=9)$ & $24(n=13)$ & 0,432 \\
$1996-1999$ & $17(n=51)$ & $13(n=36)$ & $41(n=15)$ & $<0,001$ \\
\hline $1987-1999$ & $20(n=93)$ & $17(n=51)$ & $27(n=42)$ & 0,005 \\
\hline \hline
\end{tabular}

\section{Abhängigkeit vom Alter:}

Die Korrelation mit der Unterarmlänge als relatives Maß für das Alter eines Tieres mit dem Kupfergehalt ergab keinen signifikanten Zusammenhang $(r<0,5)$. Dies gilt sowohl für das Gesamtkollektiv als auch bei ausschließlicher Betrachtung der Tiere aus dem Harz bzw. aus den harzfernen Bereichen. 


\section{Abhängigkeit vom Geschlecht:}

Der Kupfergehalt unterliegt über den Betrachtungszeitraum verteilt Schwankungen, ein einheitlicher Trend war hingegen nicht erkennbar gewesen. Zur Prüfung auf geschlechtsspezifische Unterschiede im Kupfergehalt der Haare wurde daher wiederum das gesamte Probenkollektiv von insgesamt 82 subadulten und adulten Tieren einbezogen. Juvenile Tiere wurden hierbei nicht berücksichtigt.

Für den Betrachtungszeitraum 1987-1999 konnte bei den untersuchten Zwergfledermäusen für den Kupfergehalt im Haar kein signifikanter geschlechtsspezifischer Unterschied festgestellt werden. Dies galt sowohl für die aus dem Harz stammenden Tiere als auch für die Tiere aus dem übrigen Niedersachsen (Tab. 27). Während die Medianwerte der Tiere aus dem Harz eng beieinander lagen, streuten diese bei den harzfernen Tieren stärker.

Tab. 27: Medianwerte $[\mu \mathrm{g} / \mathrm{g}]$ der Kupfergehalte im Haar von männlichen und weiblichen subadulten und adulten Zwergfledermäusen ( $P$. pipistrellus) aus dem Betrachtungszeitraum 1987-1999

\begin{tabular}{lccc}
\hline \hline \multicolumn{1}{c}{ Region } & Männchen & Weibchen & $\mathbf{p}$ \\
\hline Harz & $17(n=29)$ & $13(n=16)$ & 0,361 \\
harzfern & $31(n=26)$ & $22(n=12)$ & 0,659 \\
\hline \hline Alle Tiere & $20(n=55)$ & $18(n=27)$ & 0,328 \\
\hline \hline
\end{tabular}

\subsubsection{Nickel}

\section{Entwicklung der Nickelgehalte im Haar im Zeitraum 1987 bis 1999:}

Die Entwicklung der Nickelgehalte über den gesamten Betrachtungszeitraum ergab einen schwach abnehmenden Trend. Für den Betrachtungszeitraum 1996-1999 ergab sich für Tiere aus harzfernen Bereichen zudem ein statistisch höherer Nickelgehalt im Haar als für Tiere aus dem Harz (Tab. 28).

Tab. 28: Medianwerte der Nickelgehalte im Haar bei Zwergfledermäusen (P. pipistrellus) im Zeitraum 1987 bis 1999

\begin{tabular}{ccccc}
\hline \hline $\begin{array}{c}\text { Betrachtungs- } \\
\text { zeitraum }\end{array}$ & Alle Tiere & Tierfunde Harz & $\begin{array}{c}\text { harzferne Tier- } \\
\text { funde }\end{array}$ & $\begin{array}{c}\mathbf{p} \\
\text { Harz/harzfern }\end{array}$ \\
\hline $1987-1990$ & $2,7(\mathrm{n}=20)$ & $1,8(\mathrm{n}=6)$ & $3,0(\mathrm{n}=14)$ & 0,364 \\
$1991-1995$ & $1,9(\mathrm{n}=22)$ & $1,6(\mathrm{n}=9)$ & $2,0(\mathrm{n}=13)$ & 0,664 \\
$1996-1999$ & $1,6(\mathrm{n}=51)$ & $1,5(\mathrm{n}=36)$ & $2,7(\mathrm{n}=15)$ & 0,012 \\
\hline $1987-1999$ & $1,9(\mathrm{n}=93)$ & $1,5(\mathrm{n}=51)$ & $2,5(\mathrm{n}=42)$ & 0,001 \\
\hline \hline
\end{tabular}




\section{Abhängigkeit vom Alter:}

Die Korrelation mit der Unterarmlänge als relatives Maß für das Alter eines Tieres mit dem Nickelgehalt ergab keinen signifikanten Zusammenhang $(r<0,5)$. Dies gilt sowohl für das Gesamtkollektiv als auch bei ausschließlicher Betrachtung der Tiere aus dem Harz bzw. aus den harzfernen Bereichen.

\section{Abhängigkeit vom Geschlecht:}

Aufgrund des nachgewiesenen Trends des Nickelgehaltes in Haaren über den gesamten Untersuchungszeitraum wurde für den Vergleich zwischen den Geschlechtern bei Nickel nur der Betrachtungszeitraum 1996-1999 herangezogen. Für diesen Betrachtungszeitraum ergab sich unter Berücksichtigung aller untersuchten 44 subadulten und adulten Zwergfledermäuse kein signifikanter geschlechtsspezifischer Unterschied für den Nickelgehalt im Haar. Bei ausschließlicher Betrachtung der aus dem Harz stammenden Tiere läßt sich hingegen ein statistischer Unterschied errechnen (Tab. 29).

Tab. 29: Medianwerte $[\mu \mathrm{g} / \mathrm{g}]$ der Nickelgehalte im Haar von männlichen und weiblichen subadulten und adulten Zwergfledermäusen ( $P$. pipistrellus) aus dem Betrachtungszeitraum 1996-1999

\begin{tabular}{lccc}
\hline \hline \multicolumn{1}{c}{ Region } & Männchen & Weibchen & $\mathbf{p}$ \\
\hline Harz & $1,8(n=23)$ & $1,3(n=9)$ & 0,024 \\
harzfern & $3,2(n=9)$ & $3,2(n=4)$ & 1,000 \\
\hline Alle Tiere & $2,0(n=31)$ & $1,4(n=13)$ & 0,120 \\
\hline \hline
\end{tabular}

\subsubsection{Zink}

\section{Entwicklung der Zinkgehalte im Haar im Zeitraum 1987 bis 1999:}

Der Zinkgehalte im Säugerorganismus wird homöostatisch reguliert. Hohe Zinkgehalte im Haar treten daher nur selten auf. Zink ist aber auch dasjenige der untersuchten Elemente, welches in der Umwelt in der höchsten Konzentration vorkommt. So gelangte im Harz bei der Erzaufbereitung neben Bleisulfid vor allem Zinksulfid in großer Menge in partikulärer Form in die Umwelt. Hohe Zinkgehalte im Haar (oder besser am Haar) können auf eine exogene, durch die angewandte Waschprozedur nicht vollständig entfernte Schwermetallbelastung hindeuten. Meßserien mit Zinkgehalten von mehr als $1000 \mu \mathrm{g} / \mathrm{g}$ (> 95. Perzentil) wurden daher als Ausreißer eingestuft und komplett verworfen. 
Die Entwicklung der Zinkgehalte über den gesamten Betrachtungszeitraum ergab keinen Hinweis auf einen signifikanten abnehmenden oder zunehmenden Trend. Gleichfalls waren keine regionalen Unterschiede erkennbar (Tab. 30).

Tab. 30: Medianwerte der Zinkgehalte im Haar bei Zwergfledermäusen ( $P$. pipistrellus) im Zeitraum 1987 bis 1999

\begin{tabular}{ccccc}
\hline \hline $\begin{array}{c}\text { Betrachtungs- } \\
\text { zeitraum }\end{array}$ & Alle Tiere & Tierfunde Harz & $\begin{array}{c}\text { harzferne Tier- } \\
\text { funde }\end{array}$ & $\begin{array}{c}\mathbf{p} \\
\text { Harz/harzfern }\end{array}$ \\
\hline $1987-1990$ & $290(\mathrm{n}=20)$ & $340(\mathrm{n}=6)$ & $280(\mathrm{n}=14)$ & 0,364 \\
$1991-1995$ & $310(\mathrm{n}=22)$ & $260(\mathrm{n}=9)$ & $330(\mathrm{n}=13)$ & 0,738 \\
$1996-1999$ & $260(\mathrm{n}=51)$ & $240(\mathrm{n}=36)$ & $280(\mathrm{n}=15)$ & 0,264 \\
\hline $1987-1999$ & $270(\mathrm{n}=93)$ & $250(\mathrm{n}=51)$ & $290(\mathrm{n}=42)$ & 0,165 \\
\hline \hline
\end{tabular}

\section{Abhängigkeit vom Alter:}

Für den Zinkgehalt gilt, wie für die übrigen analysierten Schwermetalle, das keine signifikante Korrelation der Unterarmlänge als relatives $M a ß$ für das Alter eines Tieres mit dem Zinkgehalt vorlag $(r<0,5)$. Dies gilt sowohl für das Gesamtkollektiv als auch bei ausschließlicher Betrachtung der Tiere aus dem Harz bzw. aus den harzfernen Bereichen.

\section{Abhängigkeit vom Geschlecht:}

Da der Zinkgehalt wie bereits der Chrom- und Kupfergehalt während des gesamten Betrachtungszeitraumes, d.h. von 1987-1999, keinen signifikanten Änderungen unterlag, wurde zur Prüfung auf geschlechtsspezifische Unterschiede im Zinkgehalt der Haare das gesamte Probenkollektiv von insgesamt 83 subadulten und adulten Tieren einbezogen. Juvenile Tiere wurden hierbei wiederum nicht berücksichtigt. Bei den untersuchten Zwergfledermäusen konnte für den Zinkgehalt im Haar kein signifikanter geschlechtsspezifischer Unterschied festgestellt werden. Dies galt sowohl für die aus dem Harz stammenden Tiere als auch für die Tiere aus dem übrigen Niedersachsen (Tab. 31).

Tab. 31: Medianwerte $[\mu \mathrm{g} / \mathrm{g}]$ der Zinkgehalte im Haar von männlichen und weiblichen subadulten und adulten Zwergfledermäusen ( $P$. pipistrellus) aus dem Betrachtungszeitraum 1987-1999

\begin{tabular}{lccc}
\hline \hline \multicolumn{1}{c}{ Region } & Männchen & Weibchen & p \\
\hline Harz & $300(n=29)$ & $260(n=16)$ & 0,407 \\
harzfern & $300(n=26)$ & $270(n=12)$ & 0,530 \\
\hline Alle Tiere & $300(n=55)$ & $260(n=28)$ & 0,227 \\
\hline \hline
\end{tabular}



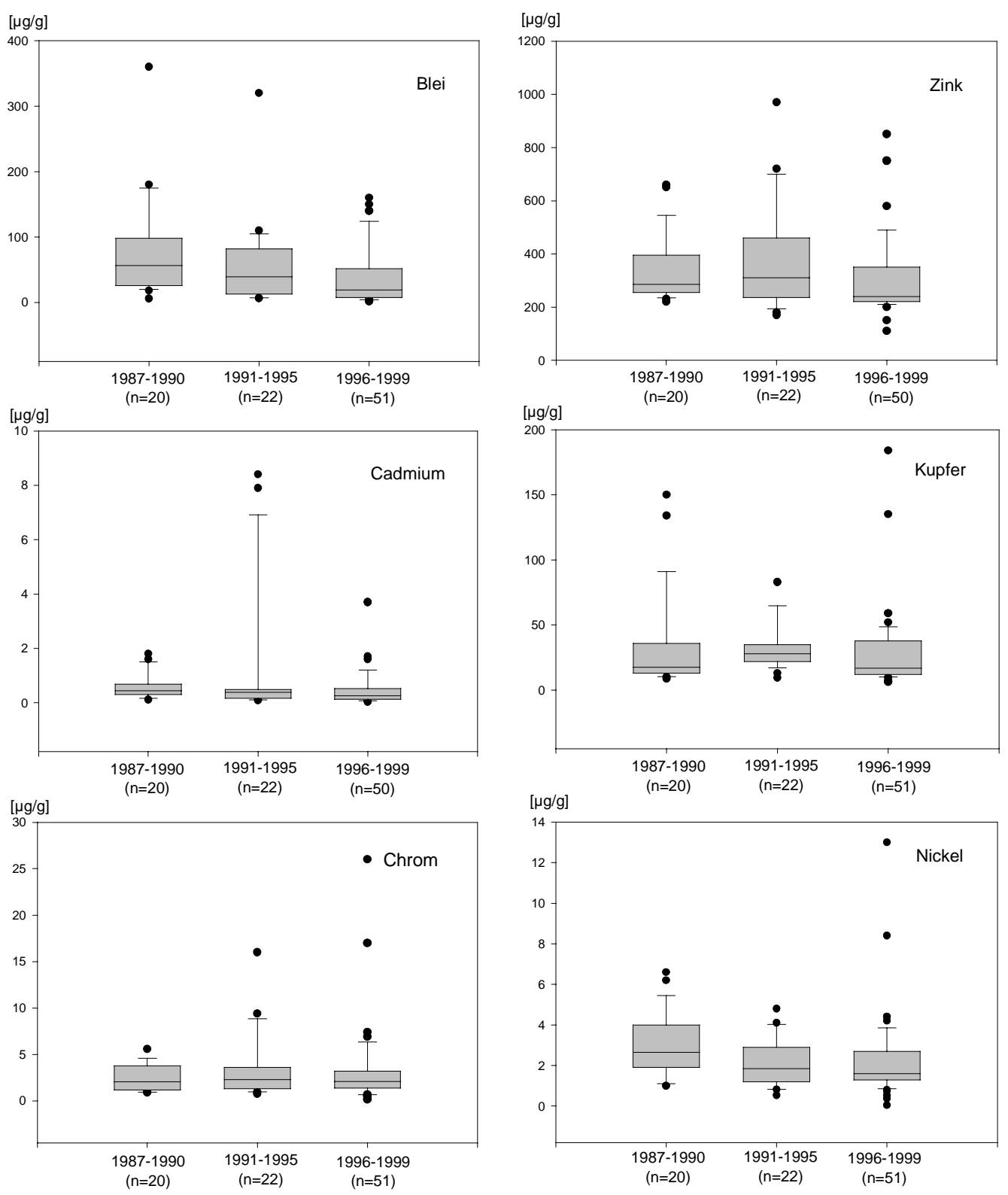

Abb. 17: Box-Plot-Diagramme der Schwermetallgehalte in Haaren von Zwergfledermäusen ( $P$. pipistrellus) für das untersuchte Gesamtkollektiv, gegliedert nach den drei Betrachtungszeiträumen. 
Tab. 32a: Statistische Kenndaten der Schwermetallgehalte im Haar der Zwergfledermäuse (P. pipistrellus) im Betrachtungszeitraum 1987-1990. Alle Meßwerte in $\mu \mathrm{g} / \mathrm{g}$

\begin{tabular}{|c|c|c|c|c|c|c|}
\hline Harz & Blei & Cadmium & Chrom & Kupfer & Nickel & Zink \\
\hline Stichprobenumfang & 6 & 6 & 6 & 6 & 6 & 6 \\
\hline Mittelwert & 66 & 0,77 & 3,0 & 30 & 2,7 & 380 \\
\hline Median & 68 & 0,52 & 2,4 & 28 & 1,8 & 340 \\
\hline Standardabweichung & 31,51 & 0,58 & 1,70 & 15,28 & 2,26 & 155,87 \\
\hline Schiefe & $-0,12$ & 0,85 & 0,81 & 0,19 & 1,22 & 1,17 \\
\hline Spannweite & 73 & 1,4 & 4,4 & 36 & 5,6 & 420 \\
\hline Minimum & 27 & 0,22 & 1,2 & 12 & 1,0 & 230 \\
\hline Maximum & 100 & 1,6 & 5,6 & 48 & 6,6 & 650 \\
\hline harzfern & Blei & Cadmium & Chrom & Kupfer & Nickel & Zink \\
\hline Stichprobenumfang & 14 & 14 & 14 & 14 & 14 & 14 \\
\hline Mittelwert & 88 & 0,54 & 2,3 & 35 & 3,2 & 320 \\
\hline Median & 56 & 0,42 & 1,9 & 17 & 3,0 & 280 \\
\hline Standardabweichung & 95,13 & 0,44 & 1,36 & 46,14 & 1,30 & 112,98 \\
\hline Schiefe & 2,02 & 2,05 & 0,55 & 2,20 & 0,84 & 2,45 \\
\hline Spannweite & 354 & 1,7 & 3,7 & 141 & 4,8 & 440 \\
\hline Minimum & 5,9 & 0,11 & 0,90 & 8,6 & 1,4 & 220 \\
\hline Maximum & 360 & 1,8 & 4,6 & 150 & 6,2 & 660 \\
\hline Nds. gesamt & Blei & Cadmium & Chrom & Kupfer & Nickel & Zink \\
\hline Stichprobenumfang & 20 & 20 & 20 & 20 & 20 & 20 \\
\hline Mittelwert & 82 & 0,61 & 2,5 & 33 & 3,0 & 330 \\
\hline Median & 57 & 0,45 & 2,0 & 18 & 2,7 & 290 \\
\hline Standardabweichung & 81,03 & 0,48 & 1,46 & 39,04 & 1,60 & 126,47 \\
\hline Schiefe & 2,37 & 1,47 & 0,67 & 2,48 & 0,76 & 1,77 \\
\hline Spannweite & 354 & 1,7 & 4,7 & 141 & 5,6 & 440 \\
\hline Minimum & 5,9 & 0,11 & 0,90 & 8,6 & 1,0 & 220 \\
\hline Maximum & 360 & 1,8 & 5,6 & 150 & 6,6 & 660 \\
\hline
\end{tabular}


Tab. 32b: Statistische Kenndaten der Schwermetallgehalte im Haar der Zwergfledermäuse (P. pipistrellus) im Betrachtungszeitraum 1991-1995. Alle Meßwerte in $\mu \mathrm{g} / \mathrm{g}$

\begin{tabular}{|c|c|c|c|c|c|c|}
\hline Harz & Blei & Cadmium & Chrom & Kupfer & Nickel & Zink \\
\hline Stichprobenumfang & 9 & 9 & 9 & 9 & 9 & 9 \\
\hline Mittelwert & 33 & 0,30 & 3,3 & 35 & 2,1 & 410 \\
\hline Median & 37 & 0,28 & 1,7 & 32 & 1,6 & 260 \\
\hline Standardabweichung & 23,96 & 0,16 & 3,30 & 19,17 & 1,24 & 282,79 \\
\hline Schiefe & 0,83 & 0,03 & 1,52 & 2,41 & 0,90 & 1,26 \\
\hline Spannweite & 76 & 0,40 & 8,7 & 63 & 3,3 & 790 \\
\hline Minimum & 6,0 & 0,09 & 0,75 & 20 & 0,82 & 180 \\
\hline Maximum & 82 & 0,49 & 9,4 & 83 & 4,1 & 970 \\
\hline harzfern & Blei & Cadmium & Chrom & Kupfer & N Nickel & ZZink \\
\hline Stichprobenumfang & 13 & 13 & 13 & 13 & 13 & 13 \\
\hline Mittelwert & 71 & 2,1 & 3,7 & 32 & 2,2 & 360 \\
\hline Median & 47 & 0,42 & 2,8 & 24 & 2,0 & 330 \\
\hline Standardabweichung & 83,13 & 3,20 & 3,92 & 19,80 & 1,22 & 140,26 \\
\hline Schiefe & 2,48 & 1,49 & 2,95 & 1,66 & 0,75 & 1,07 \\
\hline Spannweite & 313 & 8,3 & 15 & 74 & 4,3 & 520 \\
\hline Minimum & 6,8 & 0,11 & 0,96 & 9,5 & 0,53 & 170 \\
\hline Maximum & 320 & 8,4 & 16 & 83 & 4,8 & 690 \\
\hline$\overline{N d s . \text { gesamt }}$ & Blei & Cadmium & Chrom & Kupfer & "Nickel & Zink \\
\hline Stichprobenumfang & 22 & 22 & 22 & 22 & 22 & 22 \\
\hline Mittelwert & 56 & 1,3 & 3,5 & 33 & 2,2 & 380 \\
\hline Median & 40 & 0,39 & 2,3 & 28 & 1,9 & 310 \\
\hline Standardabweichung & 67,40 & 2,58 & 3,61 & 19,14 & 1,20 & 205,93 \\
\hline Schiefe & 3,09 & 2,31 & 2,44 & 1,75 & 0,74 & 1,51 \\
\hline Spannweite & 314 & 8,3 & 15 & 74 & 4,3 & 800 \\
\hline Minimum & 6,0 & 0,09 & 0,75 & 9,5 & 0,53 & 170 \\
\hline Maximum & 320 & 8,4 & 16 & 83 & 4,8 & 970 \\
\hline
\end{tabular}


Tab. 32c: Statistische Kenndaten der Schwermetallgehalte im Haar der Zwergfledermäuse (P. pipistrellus) im Betrachtungszeitraum 1996-1999. Alle Meßwerte in $\mu \mathrm{g} / \mathrm{g}$

\begin{tabular}{|c|c|c|c|c|c|c|}
\hline Harz & Blei & Cadmium & Chrom & Kupfer & Nickel & Zink \\
\hline Stichprobenumfang & 36 & 35 & 35 & 36 & 36 & 36 \\
\hline Mittelwert & 37 & 0,52 & 3,7 & 21 & 2,0 & 310 \\
\hline Median & 18 & 0,16 & 2,0 & 13 & 1,5 & 240 \\
\hline Standardabweichung & 43,61 & 0,88 & 5,13 & 29,45 & 2,04 & 158,73 \\
\hline Schiefe & 1,78 & 3,01 & 3,16 & 5,09 & 4,74 & 1,94 \\
\hline Spannweite & 159 & 3,6 & 26 & 174 & 13 & 740 \\
\hline Minimum & 1,2 & 0,07 & 0,15 & 6,30 & 0,37 & 110 \\
\hline Maximum & 160 & 3,7 & 26 & 180 & 13 & 850 \\
\hline harzfern & Blei & Cadmium & Chrom & Kupfer & N Nickel & ZZink \\
\hline Stichprobenumfang & 15 & 15 & 15 & 15 & 15 & 15 \\
\hline Mittelwert & 39 & 0,46 & 2,7 & 44 & 2,7 & 300 \\
\hline Median & 23 & 0,31 & 2,7 & 41 & 2,7 & 280 \\
\hline Standardabweichung & 42,34 & 0,37 & 1,39 & 28,18 & 1,28 & 76,69 \\
\hline Schiefe & 1,54 & 2,46 & 0,45 & 2,54 & $-0,38$ & 0,53 \\
\hline Spannweite & 137 & 1,5 & 4,9 & 127 & 4,3 & 220 \\
\hline Minimum & 3,4 & 0,16 & 0,51 & 13 & 0,08 & 200 \\
\hline Maximum & 140 & 1,6 & 5,4 & 140 & 4,4 & 420 \\
\hline$\overline{\text { Nds. gesamt }}$ & Blei & Cadmium & Chrom & Kupfer & "Nickel & Zink \\
\hline Stichprobenumfang & 51 & 50 & 50 & 51 & 51 & 51 \\
\hline Mittelwert & 37 & 0,50 & 3,4 & 28 & 2,2 & 310 \\
\hline Median & 19 & 0,25 & 2,1 & 17 & 1,6 & 260 \\
\hline Standardabweichung & 42,83 & 0,76 & 4,36 & 30,66 & 1,86 & 138,90 \\
\hline Schiefe & 1,66 & 3,32 & 3,70 & 3,59 & 4,09 & 2,05 \\
\hline Spannweite & 159 & 3,6 & 26 & 174 & 13 & 740 \\
\hline Minimum & 1,2 & 0,07 & 0,15 & 6,3 & 0,08 & 110 \\
\hline Maximum & 160 & 3,7 & 26 & 180 & 13 & 850 \\
\hline
\end{tabular}




\subsection{Schwermetallgehalte im Kot}

Die im Zeitraum 1998 bis 2000 gesammelten Kotproben wurden auf die Gehalte der acht am häufigsten vorkommenden Schwermetalle analysiert. Darüber hinaus wurden stichprobenartig Proben auf Zinn untersucht. Die gerätetechnisch bedingte Bestimmungsgrenze für Zinn ergab jedoch lediglich in 2 von 17 untersuchten Proben Meßwerte oberhalb der Bestimmungsgrenze von $3 \mathrm{mg} / \mathrm{kg}$ TS, so daß auf eine weitere Bestimmung des Zinngehaltes verzichtet wurde. Die Meßwerte sind jedoch in der Zusammenstellung der Analysenergebnisse im Anhang 7 mit enthalten. Insgesamt liegen Daten von 48 Proben aus unterschiedlichen Quartieren vor. Eine Differenzierung der Meßwerte nach der jeweiligen Art wurde nicht vorgenommen, da hierzu die Stichprobengröße als nicht ausreichend beurteilt wurde und zudem keine artspezifischen Unterschiede erkennbar waren. Bei einem Teil der Kotproben war zudem die Art unbekannt, da es sich um nur gelegentlich aufgesuchte Männchenquartiere handelte.

\subsubsection{Aktuelle Schwermetallgehalte im Fledermauskot}

Die insgesamt untersuchten 48 Kotproben lagen bis auf eine Probe aus dem Bereich Zorge/Harz, Landkreis Osterode a.H., im Vergleich zu den untersuchten Haarproben in einem engeren Variationsbereich und waren mit Ausnahme der Elemente Blei und Chrom deutlich besser normalverteilt (Abb. 18, Tab. 33).

Tab. 33: Statistische Kenngrößen der Fledermauskotuntersuchungen verschiedener Arten

\begin{tabular}{lcccccccc}
\hline \hline & $\begin{array}{c}\text { Arsen, } \\
\text { ges. }\end{array}$ & Blei & Cadmium & $\begin{array}{c}\text { Chrom, Kupfer } \\
\text { ges. }\end{array}$ & Nickel & $\begin{array}{c}\text { Queck- } \\
\text { silber }\end{array}$ & Zink \\
\hline Stichprobengröße & 47 & 47 & 47 & 47 & 47 & 47 & 46 & 47 \\
& & & & & & & & \\
Mittelwert [mg/kg TS] & 4,3 & 18 & 2,8 & 5,1 & 100 & 3,4 & 0,56 & 710 \\
Median [mg/kg TS] & 3,7 & 5,7 & 2,2 & 2,0 & 100 & 2,1 & 0,40 & 630 \\
Standardabweichung & 3,15 & 44,5 & 2,16 & 7,65 & 37,2 & 4,19 & 0,47 & 314 \\
Schiefe & 1,94 & 4,95 & 1,89 & 2,99 & 1,18 & 3,12 & 2,07 & 0,98 \\
Spannweite [mg/kg TS] & 16,5 & 279 & 9,8 & 40,2 & 175 & 21,5 & 2,0 & 1190 \\
Minimum [mg/kg TS] & 0,45 & 0,63 & 0,15 & 0,80 & 45 & 0,50 & 0,15 & 310 \\
Maximum [mg/kg TS] & 17 & 280 & 10 & 41 & 220 & 22 & 2,20 & 1500 \\
\hline \hline
\end{tabular}

Die Kotprobe 56 aus einem Quartier bei Zorge/Harz dürfte durch Staub bzw. Boden verunreinigt gewesen sein. Bei dem Quartier handelt es sich um ein altes Hüttengebäude, das sich gegenüber einem aus dem 19. Jahrhundert stammenden Schmelzofen befindet. Die hierin ermittelten Schwermetallgehalte (z.B. Blei: $4300 \mathrm{mg} / \mathrm{kg}$ TS, Kupfer: 4600 mg/kg TS, Zink: 14000 mg/kg TS, vgl. Anhang 7) entsprachen denen 
der hochbelasteten Sedimente der Harzbäche und liegen z.T. über 100-fach über den Medianwerten aller anderen untersuchten Kotproben. Diese Probe wurde daher bei der Berechnung der statistischen Kenngrößen nicht mit berücksichtigt.

\subsubsection{Veränderung der Schwermetallgehalte im Fledermauskot während der vergangenen zehn Jahre}

Aus einer vom Verfasser im Jahre 1979 entdeckten Wochenstube des Großen Mausohres wurde im Rahmen einer erneuten Begehung im Jahre 1999 neben der Entnahme von Skelettproben auch die Entnahme von Kot mittels PE-Stechrohr aus einem großen Kothaufen durchgeführt. Eine vergleichbare Probenahme wurde aus zwei Wochenstuben des Großen Mausohres bei Ahlden und Steinbergen von Dirk SCHLEGEL, Wunstorf, durchgeführt und dem Verfasser zur Untersuchung übergeben.

Die Daten aus Ahlden und Steinbergen zeigen für Blei einen Konzentrationsrückgang im Laufe des vergangenen Jahrzehnts um 50-60\%. Für die übrigen untersuchten Schwermetalle war hingegen keine deutliche Abnahme erkennbar (Tab. 34). Bei den untersuchten Kotproben aus dem Quartier Hattorf, welches Mitte der achtziger Jahre bereits erlosch, war kein eindeutiger Trend erkennbar. Sowohl eine Kotprobe aus dem eingangsnahen Bereich als auch eine zweite mittels PE-Stechrohr entnommene Kotprobe im hinteren Teil des Quartieres zeigte an der Haufenoberfläche geringfügig höhere Schwermetallgehalte als im unteren Bereich der Kothaufen.

Tab. 34: Ergebnisse der Analyse von Kothaufen aus Wochenstuben des Großen Mausohres (M. myotis). Alle Meßwerte in $\mathrm{mg} / \mathrm{kg}$ TS

\begin{tabular}{lcccccc}
\hline \hline Element & \multicolumn{2}{c}{ Hattorf/Harz } & \multicolumn{2}{c}{ Ahlden } & \multicolumn{2}{c}{ Steinbergen } \\
& vor 1980 & $\mathbf{1 9 8 6}$ & vor 1990 & $\mathbf{1 9 9 9}$ & $\mathbf{1 9 8 9}$ & $\mathbf{1 9 9 9}$ \\
\hline Arsen, ges. & 17 & 0,57 & $<3,3$ & $<3,2$ & $<3,2$ & $<2,8$ \\
Blei & 16 & 23 & 12 & 6,5 & 15 & 6,2 \\
Cadmium & 10 & 4,5 & 3,6 & 3,2 & 2,4 & 2,0 \\
Chrom, ges. & 1,2 & $<3,1$ & $<3,3$ & $<3,2$ & $<3,2$ & $<2,8$ \\
Kupfer & 86 & 55 & 78 & 75 & 85 & 63 \\
Nickel & 1,3 & $<3,1$ & $<1,6$ & $<1,6$ & $<1,6$ & 1,4 \\
Quecksilber & 0,23 & $<0,2$ & 0,22 & 0,24 & 0,28 & 0,28 \\
Zink & 410 & 360 & 510 & 460 & 420 & 430 \\
\hline \hline
\end{tabular}

Theoretisch wäre eine Zunahme des Schwermetallgehaltes in den tieferen (älteren) Schichten der Kothaufen auch durch den länger anhaltenden mikrobiellen Umsatz des Materiales und damit einer stärkeren Mineralisierung erklärbar. Während hierbei der Kohlenstoffanteil veratmet wird, verbleiben die Schwermetalle in unveränderter Form und Menge in dem restlichen Kotmaterial. Die Schwermetallzunahme in den älteren Schichten hätte dann aber für alle Elemente annähernd gleich sein müssen. 
Zur Absicherung erfolgte die Bestimmung des Glühverlustes an den zuvor getrockneten Kotproben als Maß für den enthaltenen organischen Kohlenstoffanteil. Der hierbei feststellbare Unterschied zwischen Proben von der Haufenoberfläche und der Sohle der Kothaufen betrug maximal 3,7 Gew.-\% und lag in den unteren Kotschichten in drei von vier untersuchten Proben zudem höher (Tab. 35). Dieses widerspricht der Annahme, das die unteren Schichten der bis zu $80 \mathrm{~cm}$ hohen Kothaufen stärker mineralisiert sind als die oberflächennahen Lagen. Viel eher ist anzunehmen, daß aufgrund der hohen Harnstoffgehalte in tieferen Lagen eine verminderte mikrobiologische Umsetzung infolge sterilisierender Effekte des Harnstoffs erfolgt.

Tab. 35: Ergebnisse der Glühverlustbestimmungen von der Oberfläche und der Sohle der Kothaufen aus Wochenstuben des Großen Mausohres (M. myotis). Alle Meßwerte in Gew.-\% TS

\begin{tabular}{lcccc}
\hline \hline & \multicolumn{2}{c}{ Hattorf/Harz } & Ahlden & Steinbergen \\
Probennr. & FMK 21 & FMK 22 & FMK 32 & FMK 41 \\
\hline oberer Bereich & 79,5 & 87,7 & 88,0 & 92,7 \\
unterer Bereich & 81,9 & 91,4 & 89,2 & 91,8 \\
\hline Differenz & 2,4 & 3,7 & 1,2 & $-0,9$ \\
\hline \hline
\end{tabular}
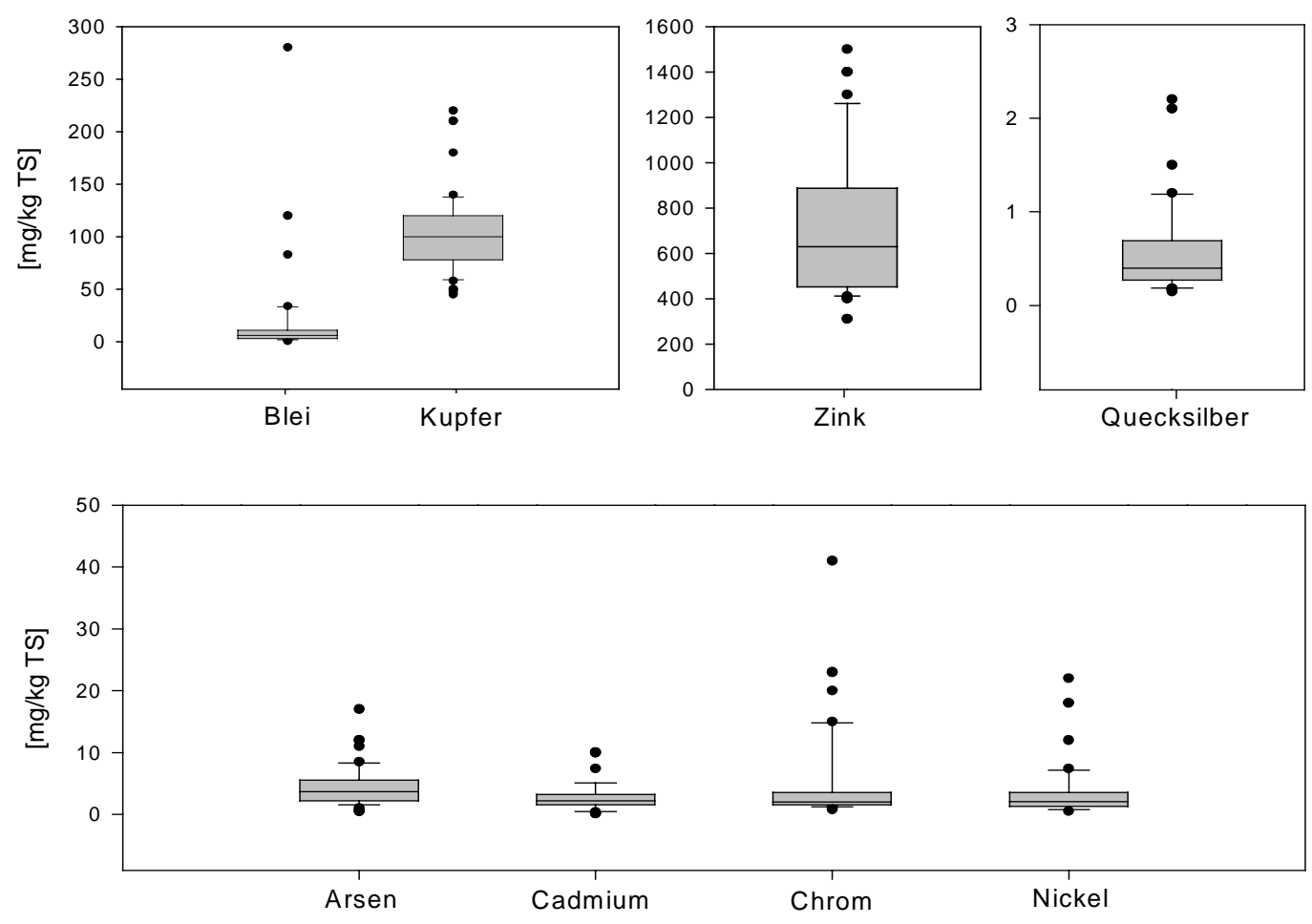

Abb. 18: Schwermetallverteilung in 47 von 48 untersuchten Fledermauskotproben. 


\subsection{Nachweis von Platin}

Von den Platingruppenelementen konnte aufgrund des hohen analytischen Aufwandes lediglich Platin stichprobenartig und exemplarisch an wenigen Proben analysiert werden. Die Durchführung der Analysen erfolgte durch Herrn Dr. ALT, Institut für Spektrochemie, Dortmund, mittels Voltammetrie.

In den drei untersuchten Knochenproben ergab sich kein gesicherter Nachweis von Platin. Alle Meßwerte lagen unterhalb oder an der Bestimmungsgrenze.

In allen untersuchten Kotproben sowie in drei der vier untersuchten Haarproben der Zwergfledermäuse ergaben sich hingegen Nachweise von Platin, wobei die höchsten Platingehalte in Proben aus Innenstadtgebieten ermittelt wurden (Tab. 36).

Tab. 36: Platingehalte in Kot-, Knochen- und Haarproben von Fledermäusen

\begin{tabular}{cllc}
\hline $\begin{array}{c}\text { Proben } \\
\text { Nr. }\end{array}$ & \multicolumn{1}{c}{ Art } & \multicolumn{1}{c}{ Herkunft } & $\begin{array}{c}\text { Platin } \\
\text { [ng/g TS }\end{array}$ \\
\hline Kot: & & & \\
\hline 5 & Fransenfledermaus & Stadtzentrum Nienburg/Weser & 1,7 \\
9 & Bechsteinfledermaus & $\begin{array}{l}\text { Wunstorf, Wochenstube an der B 214 } \\
\text { Stadtzentrum Wunstorf }\end{array}$ & 1,0 \\
12 & Zwergfledermaus & & 6,8 \\
Unterarmknochen: & Hwergfledermaus & Hannover-Kleefeld & \\
\hline 96001 & Zwergfledermaus & Stadtzentrum Hannover & rd. 0,7 \\
96003 & Zwergfledermaus & & $<0,5$ \\
98058 & & Stadtzentrum Hannover & $<0,5$ \\
& & B 241, Osterode-Clausthal & \\
Haare: & & Osterode am Harz, OT Freiheit & 2,6 \\
96003 & Zwergfledermaus & Stadtgebiet Osterode am Harz & 1,9 \\
97001 & Zwergfledermaus & & 1,9 \\
98020 & Zwergfledermaus & & $<, 5$ \\
98058 & Zwergfledermaus & & \\
\hline \hline
\end{tabular}

*) für Haare bezogen auf $50 \%$ Feuchtegehalt 


\section{Diskussion}

\subsection{Exposition, Bioverfügbarkeit und Toxikokinetik der Schwermetalle}

\section{Blei:}

In der Natur kommt Blei primär als Bleisulfid (Bleiglanz, PbS) oft in enger Vergesellschaftung mit Zink- und Kupfererzen vor. Metallisches Blei findet Verwendung in Autobatterien, als Lagermetall sowie als Schrotblei für Jagdzwecke. Organobleiverbindungen waren bis 1987 in Deutschland als Antiklopfmittelzusatz für Vergaserkraftstoffe von wesentlicher Bedeutung (vgl. Kap. 4.4). Hieraus resultieren auch die ubiquitär vorhandenen Bleidepositionen in allen Umweltkompartimenten. Infolge der Erzaufbereitung im Harz gelangten große Mengen bleihaltiger Hüttenrauch, vor allem als Bleioxid, in die Atmosphäre. BURKARD (1999) errechnete für die im südlichen Harzvorland gelegene Beiersteinsenke eine Bleideposition bis zu $1150 \mathrm{mg} / \mathrm{m}^{2} \mathrm{a}$ während der Hauptbergbauphase im Spätmittelalter. Der geogene Eintrag kann hingegen mit $10 \mathrm{mg} / \mathrm{m}^{2} \mathrm{a}$ angenommen werden. Neben der Bleiverfrachtung über die Atmosphäre gelangten bei der Erzaufbereitung zudem bleierzhaltige Ton- und Schluffbestandteile (Bleisulfide) in die Fließgewässer (vgl. Kap. 4.5). Dies führte zu einer erheblichen Bleianreicherung in den Sedimenten der Überschwemmungsbereiche (LINDORFER 1997).

Im Boden deponiertes Blei ist wenig bioverfügbar. Sinkt der pH-Wert jedoch unter 4, so nimmt die Löslichkeit des Bleis und damit die physiologische Wirksamkeit wesentlich zu (BACHMANN et al. 1998). Durch Säugetiere oral aufgenommenes Blei kann daher im Magen-Darmtrakt infolge des um 2 liegenden $\mathrm{pH}$-Wertes der Magensäure zu $5-10 \%$ resorbiert werden.

Inhalativ aufgenommenes Blei wird in der Lunge in Abhängigkeit von der Partikelgröße retiniert und zu rund $50 \%$ resorbiert (WIRTH und GLOXHUBER 1994). Die Bindung des aufgenommenen Bleis erfolgt zu $90 \%$ an die roten Blutkörperchen. Die Anreicherung im Leber und im Nierengewebe hingegen ist gering. Blei wird im Knochengewebe anstelle von Calcium als Bleiphosphat oder Calcium-Bleiphosphat deponiert. Physiologisch ist das so deponierte Blei nicht mehr relevant. Infolge Erkrankungen kann es jedoch wieder freigesetzt werden und zu Intoxikationen führen. Der Knochenbleigehalt liefert Informationen über die Bleibelastung im Wachstumsalter. Haarbleigehalte wie auch Blutbleigehalte können hingegen als Indikator für erhöhte Bleiaufnahmen während der zurückliegenden Monate bei allen Altersgruppen angesehen werden. 


\section{Cadmium:}

Cadmium kommt in der Natur als Cadmiumsulfid (Cadmiumblende, CdS) sowie als Cadmiumcarbonat in Vergesellschaftung mit Blei-Zinkerzen vor. Im Vergleich zu Blei und Zink liegt der Siedepunkt des Cadmiums mit $767^{\circ} \mathrm{C}$ rund $1000^{\circ}$ unter dem von Blei und $150^{\circ}$ unter dem von Zink. Bei der Verhüttung von Blei-Zinkerzen aus dem Bereich des Harzes gelangten infolge des niedrigen Siedepunktes daher große Mengen von Cadmium, primär als Cadmiumoxid, in die Atmosphäre und letztendlich mit den Niederschlägen in den Waldboden. Technisch wurde Cadmium als Korrosionsschutz auf Eisenmetalle aufgebracht (cadmieren) und diente als Stabilisator für PVC sowie als Pigment in Farben und Glasuren. Die Verwendung von Cadmium in diesen Einsatzfeldern ist aufgrund der bekannten Toxizität stark rückläufig. Heute kommt Cadmium vorrangig Bedeutung bei der Herstellung von Akkumulatoren zu.

Die Anreicherung von Cadmium im Boden erfolgt vorrangig durch $\mathrm{pH}$-abhängige Adsorption an (Ton-)Partikel, wobei Eisenoxiden neben Mangan- und Aluminiumoxiden eine besondere Bedeutung zukommen (SCHULTE und BLUM 1997). Huminstoffkomplexe besitzen hingegen eine nur geringe Stabilität. Infolge der schwach ausgeprägten und fast vollständig reversiblen Bindungseigenschaften am Bodenkorn ist Cadmium vergleichsweise leicht für Pflanzen und Bodenorganismen verfügbar und gilt als eines der am stärksten bioakkumulierenden Schwermetalle (BACHMANN et al. 1998). Bei sinkenden pH-Werten steigt die Mobilität von Cadmium im Boden bis zu $60 \%$ an. Infolge der geogen bedingten sauren Standortverhältnisse im Bereich des Harzes kommt damit der Bioakkumulation von Cadmium eine wesentliche Bedeutung zu.

Oral aufgenommenes Cadmium wird zu etwa $6 \%$ im Magen-Darmtrakt resorbiert (WIRTH und GLOXHUBER 1994). Infolge hoher Bindungsintensität an niedermolekularen Proteinen des Blutplasmas erfolgt eine langanhaltende Resorption in Nieren und Leber, wobei nach Untersuchungen von KolLMEIER et al. (1985) beim Menschen die Anreicherung in der Niere weitaus höher ist als in der Leber. Cadmium weist nach KALBERLAH (1999) keine essentielle Funktion im Säugetierorganismus auf. Dem entgegen stehen Untersuchungen von ANKE, die eine Essentialität vermuten lassen (ANKE 1993).

\section{Chrom:}

In der Natur findet sich Chrom fast ausschließlich in der Oxidationsstufe +Ill als Chromeisenstein (Chromit). Chrom-VI-Verbindungen entstammen überwiegend anthropogenen Quellen. Im Gegensatz zu den Chrom-III-Verbindungen sind zahlreiche Chrom-VI-Verbindungen (Chromate) sehr gut wasserlöslich. Technische Bedeutung haben Chromverbindungen bei der Veredlung von Stählen, in der Galvanik, im Holzschutz, als Pigment in Farben sowie in der Gerberei. 
In schwach bis stark alkalischen Böden mit guter Durchlüftung kann es zur Oxidation von Chrom-III zu Chrom-VI kommen. Der Chrom-VI-Gehalt im Boden wird nach BACHMANN et al. (1998) auf maximal $10 \%$ des Gesamtchromgehaltes geschätzt. Aufgrund des geringen Löslichkeitsproduktes 3-wertiger Chromverbindungen ist die Bioakkumulation von Chrom als gering zu beurteilen. Nur bei Anwendung und Handhabe technischer Chrom-VI-Produkte können neben Überempfindlichkeitsreaktionen auch Vergiftungssymptome beobachtet werden. Die Anreicherung von Chrom im Säugetierorganismus ist gering und beträgt nach SCHÄFER et al. (1997) lediglich $0,5 \%$ (Cr-III) bzw. $2 \%$ (Cr-VI), wobei in der Leber mehr angereichert wird als in den Nieren. Im Organismus erfolgt rasch eine Reduktion zu Chrom-III.

\section{Kupfer:}

Kupfer kommt in der Natur überwiegend als Kupferkies $\left(\mathrm{CuFeS}_{2}\right)$ vor, aber auch als Metall (gediegen) und in Form von Hydroxiden, Karbonaten und Sulfaten. Es zählt zu den technisch wichtigsten Metallen und ist für den Stoffwechsel der Säugetiere das wichtigste Spurenelement. Lösliche Kupfersalze werden in der Landwirtschaft als Fungizid und Algizid eingesetzt. Im Holzschutz finden Kupfersalze als Imprägniersalz mit fungizider Wirkung Anwendung.

Kupfer wird im Boden in hohem Maße an die organische Fraktion im Boden gebunden und hierin angereichert.

Im Gastrointestinaltrakt von Säugetieren wird im Vergleich zu anderen Schwermetallen mit 30-50 \% der oral aufgenommenen Kupfermenge ein hoher Anteil resorbiert. Der Kupfergehalt im Körper ist homöostatisch reguliert, die Ausscheidung erfolgt mit dem Urin über die Nieren. Infolge der homöostatischen Regelung ist die Bioakkumulation von Kupfer gering. Gleichfalls sind Vergiftungen oder starke Kupferanreicherungen im Gewebe selten und treten nur bei hoher Kupferingestion oder bei krankhafter Stoffwechselstörung (z.B. Morbus-Wilson) auf (SCHÄFER et al. 1997). Die Kupferanreicherung im Lebergewebe ist etwa doppelt so hoch wie im Nierengewebe.

\section{Nickel:}

Nickel kommt in der Natur überwiegend als Oxid, Sulfid oder Silikat vor. Als Legierungsmittel zur Stahlveredlung besitzt Nickel, wie Chrom, große technische Bedeutung. Nickel zählt infolge der hohen technischen Bedeutung und des damit verbundenen vielfältigen Einsatzes nach Blei zu dem am stärksten im Niederschlag vertretenen Schwermetall. SCHULTE und BLUM (1997) vermuten daß bereits in diesem Jahrzehnt Nickel das Schwermetall sein wird, welches am höchsten mit den Niederschlägen in den Boden eingetragen wird. Aufgrund geringerer Bindungsfähigkeit von Nickel an die organische Bodenfraktion im Vergleich zu Kupfer kann Nickel unterhalb eines $\mathrm{pH}$-Wertes von 6,5 wesentlich leichter ausgeschwemmt werden. Oberhalb eines pH-Wertes von 6,7 nimmt die Wasserlöslichkeit von Nickel (als Nickelhydroxid) stark ab. 
Die Bioakkumulation von Nickel ist gering. Hohe Nickelaufnahmen führen primär zur Störung des Zinkstoffwechsels. Darüber hinaus erfolgt bei chronischer Nickelbelastung die Ausbildung einer Nickeldermatitis.

\section{Zink:}

Zink kommt in der Natur überwiegend in Form von Zinksulfid (Zinkblende ZnS) vor. Es zählt zu den wichtigsten industriell verwendeten Metallen. Neben der eigentlichen Verwendung von metallischem Zink, z.B. zur Oberflächenveredlung und als Legierungsmetall, findet sich Zink in allen Gummiprodukten zur Abbindung des der Gummi-Rohmasse zugesetzten überschüssigen Schwefels. Der Reifenabrieb stellt daher auch eine der wichtigsten anthropogenen Zinkquellen dar.

Zink zählt neben Cadmium und Nickel zu den mobilen Schwermetallen im Boden. Die Adsorption von Zink im Boden ist u.a. abhängig vom pH-Wert und dem Gehalt an Eisen-, Mangan- und Aluminiumhydroxiden

Für Säugetiere stellt Zink neben Kupfer das wichtigste Spurenelement dar. Der Zinkgehalt im Körper wird homöostatisch reguliert. Die Bioakkumulation von Zink im Säugetierorganismus ist daher sehr gering. Chronische gewerbliche Zinkvergiftungen sind nicht bekannt (WIRTH und GLOXHUBER 1994). Bei Zinkmangel treten hingegen schwere Haar- und Gefiederschäden sowie großflächige Hautschäden auf.

\subsection{Aussagefähigkeit und Interpretation der Schwermetallmessungen}

\subsubsection{Differenzierung zwischen endogener und exogener Schwermetall- belastung}

Grundsätzlich stellt sich bei allen Rückstandsanalysen die Frage, inwieweit die ermittelten Schadstoffgehalte biologisch relevant sind. Schwermetallhaltige Partikel auf der Körperoberfläche oder dem Haar führen noch nicht zu einer Beeinflussung der physiologischen Prozesse im Organismus. Physiologisch wirksam können nur solche Substanzen sein, die in den Stoffwechsel der Organismen eingreifen bzw. diesen beeinflussen.

Bei der Untersuchung des Knochengewebes, aber auch der inneren Organe, können die nachgewiesenen Schwermetallgehalte nur als endogene Belastung beurteilt werden. Die Bestimmung des Bleigehaltes von linkem und rechtem Unterarmknochen am selben Tier ergab jeweils eine gute Übereinstimmung, wobei mit Verringerung der Probeneinwaage, d.h. bei jüngeren Tieren, die Streuung der Meßwerte leicht zunahm. Exogene Schwermetallbelastungen, die in diesem Falle nur durch Laborkontamination bei der Probenaufbereitung hätten entstehen können, sind somit nicht von Bedeutung. Bei Vorliegen derartiger Kontaminationen wäre eine stärkere Streuung der Meßwerte innerhalb eines Tieres zu erwarten gewesen. 
Nicht vollkommen auszuschließen ist eine Einwanderung von Metallionen von außen in das Knochengewebe bei den untersuchten Skelettfunden aus der Wochenstube in Hattorf. SCHUTKOWSKI (1998) diskutiert eine solche postmortale Änderung des Bleigehaltes im Knochengewebe im Zusammenhang mit der Untersuchung von menschlichen Skelettfunden. Je nach umgebendem Milieu ist eine An- oder Abreicherung der mineralischen Bestandteile oder von einzelnen mineralischen Fraktionen im Knochengewebe möglich. Zur Verringerung dieser postmortalen Beeinflussung wurden daher ausschließlich Skelettreste analysiert, die auf den Kothaufen oder auf dem Holz der Dachkonstruktion lagen. Da die Kothaufen oberflächlich aufgrund der wasserdichten Dachkonstruktion stark abgetrocknet waren, wird die Wahrscheinlichkeit einer exogenen postmortalen Einwanderung von Blei in das Knochengewebe für vernachlässigbar erachtet.

In allen anderen untersuchten Fällen waren die Unterarmknochen noch von der Flughaut bedeckt. Auch standen die Totfunde nur kurzzeitig mit dem Erdboden in Berührung, wie an dem Verwesungsgrad erkennbar war.

Für die Untersuchung der Haarmatrix stellt sich die Situation hingegen anders dar. Die Einlagerung von inkorporierten Schadstoffen in die Haarmatrix erfolgt nur im Bereich der eigentlichen Haarwachstumszone im Haarwurzelbereich (RoBBINS 1988, KaTZ und CHATT 1988, KRAUSE und CHUTSCH 1987, KIJEWSKI 1993). Gleichwohl kann aber auch eine Einlagerung von Schadstoffen in die Haarmatrix von außen erfolgen.

KIJEWSKI und LANGE (1977) untersuchten mittels Elektronenstrahlmikrosonde die Wanderungskinetik von Metallionen, die von außen auf das Haar aufgebracht wurden. Bereits nach 2-stündiger Einwirkzeit betrug die Eindringtiefe der Metallionen etwa 2/3 des Haarradius. Nachfolgendes Waschen der Haare (30 min mit destilliertem Wasser) führte zwar zu einer Reduzierung der Metallgehalte im äußeren Haarbereich, es kam aber auch zu einer weiteren Verlagerung der Metallionen in Richtung Haarmittelachse.

KIJEWSKI (1993) berichtet von einem Fall, bei dem $1300 \mu \mathrm{g} / \mathrm{g}$ Haarblei bei einer Patientin nachgewiesen wurde. Der Normalbereich (Medianwert der bundesdeutschen Bevölkerung, ermittelt aus 3817 Untersuchungen) beträgt bei 25 - 69 Jahre alten Personen 0,9 $\mu \mathrm{g} / \mathrm{g}$, das arithmetische Mittel liegt bei 2,26 $\mu \mathrm{g} / \mathrm{g}$ (KRAUSE et al. 1997). Im Blutbleigehalt zeigte die Patientin hingegen keine Auffälligkeit $(7 \mu \mathrm{g} / \mathrm{dl})$. Somit war hier eine exogene Bleibelastung des Haares zu vermuten. Durch schonendes Waschen war jedoch keine Reduzierung des Haarbleigehaltes zu erreichen. Nachprüfungen ergaben, daß die Patientin ein stark bleihaltiges Haarfärbemittel benutzt hatte.

Verantwortlich für die irreversible Einlagerung von Metallionen in die Haarmatrix sind vermutlich die Mikroporen der Keratinfasern im Haar. Durch Diffusion können Substanzen, deren lonenradius dem Porenradius der Keratinfaser entspricht, in diese 
hineindiffundieren. In der Keratinfaser erfolgt sodann eine Fixierung über Wasserstoffbrückenbindungen (ROBBINS 1988). KIJEWSKI (1993) zeigte, daß nach Erweiterung der Porenradien durch dreimaliges Erhitzen auf $160^{\circ} \mathrm{C}$ und Dehnung des Haares der Quecksilberanreicherungsfaktor von 0,15 auf 5-7 erhöht wurde.

In der Literatur finden sich zum Thema der Haarreinigung umfangreiche methodische Vorschläge, wobei teilweise mit Detergenzien, Säuren und Laugen gearbeitet wurde. In zahlreichen Fällen wurden Wasser und organische Lösungsmittel zur Haarreinigung verwendet. Eine umfassende Zusammenstellung zum Thema Haarreinigung liefert KIJEWSKI (1993).

Auch im Rahmen der vorliegenden Untersuchung des Haares von Fledermäusen erfolgte eine Haarreinigung vor dem eigentlichen Probenaufschluß. Bei dem ausgewerteten Fundmaterial handelte es sich ausschließlich um Totfunde. Das Fell der Tiere war z.T. mit Kot verklebt oder von Milben befallen. Anhaftende Bodenpartikel waren mechanisch ebenfalls nur unzureichend zu entfernen. Die auf der Haarmatrix anhaftenden Partikel hätten unweigerlich eine zusätzliche starke Streuung der Meßwerte verursacht und wären nicht repräsentativ für die Erfassung des endogenen Schwermetallstatus der Fledermäuse gewesen.

Die angewandte IAEA-Reinigungsmethode wurde als vergleichsweise mildes Reinigungsverfahren angesehen und daher den anderen in der Literatur beschriebenen Techniken vorgezogen. Die Reinigung erfolgte ausschließlich bei Raumtemperatur, jeder Waschschritt dauerte lediglich $10 \mathrm{~min}$. Hierdurch wurde Wanderungsgeschwindigkeit von Metallionen im Gegensatz zu den beschriebenen mehrstündigen Reinigungsprozeduren bei z.T. erhöhten Temperaturen niedrig gehalten.

Die im Boden enthaltenen Metalle liegen zudem überwiegend in oxidischer oder sulfidischer Form vor. Das hierdurch bedingte niedrige Löslichkeitsprodukt verhindert die Ausbildung hoher Metallionenkonzentrationen, sofern keine Komplexbildner oder Säuren bei der Waschprozedur verwendet werden. Der methodische Fehler durch die Beeinflussung der Haarmatrix infolge der angewandten Waschprozedur wird daher als weitaus geringer beurteilt als bei einem völligem Verzicht auf eine Haarreinigung.

\subsubsection{Vergleich der Meßwerte mit Literaturangaben}

In der internationalen Literatur finden sich zur Schwermetallbelastung von Fledermäusen nur sehr vereinzelt Angaben. Oft umfassen sie zudem nur geringe Stichprobenumfänge, methodisch problematisch zu bewertende Untersuchungstechniken oder beschreiben Arten, die in Mitteleuropa nicht heimisch sind. Im nachfolgenden soll daher eine Übersicht über die vorliegenden Daten zur Schwermetallbelastung der Fledermäuse gegeben werden, um diese mit den eigenen Messungen vergleichen zu können. 


\subsubsection{Microchiroptera}

Neuere Daten zur Schwermetallbelastung mitteleuropäischer Fledermausarten finden sich bei NAGel (1989), Leitner und Vogel (1993), Streit und NAGel (1993a, 1993b) GereLL \& GeRELL (1993) und ZYRNICKI et al. (1996).

LEITNER und VOGEL (1993) kommen in ihrer Studie über Fledermäuse als Bioindikatoren zu dem Ergebnis, daß aufgrund der Oberflächenkontamination der Totfunde durch Staub diese nur bedingt zur Indikation ökotoxikologisch relevanter Schwermetallbelastungen geeignet sind. Die Schwermetalluntersuchungen basieren auf insgesamt drei Großen Mausohren (Myotis myotis), die alle aus derselben Wochenstube stammten. Der Todeszeitpunkt lag etwa eine Woche zurück. Die Tiere wurden vor dem Aufschluß nicht bis zur Gewichtskonstanz getrocknet, so daß bereits die einzelnen Meßwerte dieser Untersuchungsreihe nicht miteinander vergleichbar sind. Im Rahmen der Probenvorbereitung wurden die Tiere mit flüssigem Stickstoff tiefgefroren und sodann als ganzes aufgemahlen. Der eigentliche Probenaufschluß erfolgte mit Salpetersäure (Suprapur) als Druckaufschluß. Die Messungen erfolgten mittels ICP und AAS. Bei Betrachtung der Meßwerte fällt auf, daß die untersuchte Frühgeburt mit Abstand den höchsten Schwermetallgehalt aufweist (Tab. 37). Aufgrund der Vorgehensweise bei der Probenaufbereitung (fehlende Trocknung bis zur Gewichtskonstanz, Ganztieraufschluß) lassen diese Daten keinen wirklichen Rückschluß auf die Schwermetallbelastung der untersuchten Tiere zu.

NAGEL (1989) berichtete über erste Schwermetalluntersuchungen an insgesamt zehn Fledermäusen aus fünf verschiedenen Taxa von Totfunden aus Darmstadt (Hessen). Analysiert wurden die Karkassen, d.h. die Tiere ohne Organe und Magen-Darmtrakt. Die Tiere wurden getrocknet und pulverisiert. Angaben zum Aufschlußverfahren und zur Bestimmungstechnik fanden sich nicht. NAGEL vergleicht die Meßergebnisse mit denen gesunder Menschen und stellt fest, daß ,...kein großer Unterschied zu erkennen (ist). Das bedeutet: Eine große Belastung der Fledermäuse mit Schwermetallen ist nicht vorhanden; Schwermetalle kommen somit als Grund für den Rückgang der Fledermäuse nicht in Frage. "Die von NAGEL publizierten Meßwerte (Tab. 38) entsprechen in der Größenordnung denen späterer publizierter Daten von NAGEL (STREIT und NAGEL 1993a, b).

An einem größeren Probenkollektiv von insgesamt 45 Tieren aus sechs Taxa führten StREIT und NAGEL (1993a) ebenfalls Schwermetalluntersuchungen am Ganztieraufschluß (35 Tiere), aber auch an Organ-, Haar- und Nagelproben (10 Tiere) durch. Alle Tiere wurden im Zeitraum April 1981 bis Juni 1989 in Hessen (Deutschland) gesammelt. Die Messungen erfolgten nach Trocknung bei $60^{\circ} \mathrm{C}$ mittels AAS und Feststoffprobenaufgabe oder nach Naßaufschluß in konzentrierter Salpetersäure. Die Messungen der Ganztieraufschlüsse ergaben bei den sogenannten Hausfledermäusen (Eptesicus serotinus, Pipistrellus pipistrellus) insbesondere für Blei und Chrom deutlich höhere Gehalte als bei den waldbewohnenden Arten (Myotis daubentoni, Myotis mystacinus, Nyctalus noctula, Plecotus auritus; Tab. 39). Dieses Ergebnis spiegelte sich auch in den Untersuchungen von Knochenproben (Femur) 
wider. Im Femur von E. serotinus wurden Bleigehalte zwischen $17 \mu \mathrm{g} / \mathrm{g}$ TS und $34,8 \mu \mathrm{g} / \mathrm{g}$ TS ermittelt, in allen anderen Arten lagen die Bleigehalte hingegen nur zwischen $1,5 \mu \mathrm{g} / \mathrm{g}$ TS und $8 \mu \mathrm{g} / \mathrm{g}$ TS. Die von NAGEL publizierten Knochenbleigehalte liegen damit in der gleichen Größenordnung wie die im Rahmen dieser Untersuchung für Zwergfledermäuse und Großes Mausohr am Radius ermittelten Werte.

Tab. 37: Ergebnisse von Schwermetalluntersuchungen an Ganzkörperaufschlüssen vom Großen Mausohr ( $M$. myotis) aus einer Wochenstube im südlichen Burgenland (Österreich) durch LEITNER und VOGEL (1993)

\begin{tabular}{lcccc}
\hline \hline & Einheit & Tier 1 & Tier 2 & Tier 3 \\
\hline Alter & & Frühgeburt & 1 Woche & 5 Wochen \\
Erhaltungszustand & & lufttrocken & lufttrocken & frischtot \\
Blei & {$[\mu \mathrm{g} / \mathrm{g} \mathrm{FS}]$} & 3,9 & 2,3 & 1,1 \\
Cadmium & {$[\mu \mathrm{g} / \mathrm{g} \mathrm{FS}]$} & - & 0,2 & $<0,05$ \\
Kupfer & {$[\mu \mathrm{g} / \mathrm{g} \mathrm{FS}]$} & 33,5 & 8,4 & 3,5 \\
Quecksilber & {$[\mu \mathrm{g} / \mathrm{g} \mathrm{FS}]$} & 2,3 & 0,8 & 0,4 \\
Zink & {$[\mu \mathrm{g} / \mathrm{g} \mathrm{FS}]$} & 288 & 121 & 41 \\
\hline \hline
\end{tabular}

Tab. 38: Ergebnisse von Schwermetalluntersuchungen an Ganzkörperaufschlüssen. Mittelwert von insgesamt 10 Fledermaustotfunden aus 5 Taxa aus Darmstadt (Hessen/Deutschland). Nach NAGEL (1989)

\begin{tabular}{lcc}
\hline \hline Element & $\begin{array}{c}\text { mittlere } \\
\text { Konzentration }\end{array}$ & Einheit \\
\hline Blei & 12,7 & $\mathrm{mg} / \mathrm{kg} \mathrm{TS}$ \\
Cadmium & 0,16 & $\mathrm{mg} / \mathrm{kg} \mathrm{TS}$ \\
Chrom & 5,2 & $\mathrm{mg} / \mathrm{kg} \mathrm{TS}$ \\
Kobalt & 0,9 & $\mathrm{mg} / \mathrm{kg} \mathrm{TS}$ \\
Kupfer & 13,6 & $\mathrm{mg} / \mathrm{kg} \mathrm{TS}$ \\
Nickel & 2,9 & $\mathrm{mg} / \mathrm{kg} \mathrm{TS}$ \\
Zink & 207 & $\mathrm{mg} / \mathrm{kg} \mathrm{TS}$ \\
\hline \hline
\end{tabular}

Tab. 39: Ergebnisse von Schwermetalluntersuchungen an Ganzkörperaufschlüssen von Fledermaustotfunden aus Hessen (Deutschland). Nach STREIT und NAGEL (1993a). Alle Meßwerte in $\mu \mathrm{g} / \mathrm{g}$ TS

\begin{tabular}{lcccccc}
\hline \hline & $\begin{array}{c}E . \\
\text { serotinus }\end{array}$ & $\begin{array}{c}\text { P. pipi- } \\
\text { strellus }\end{array}$ & $\begin{array}{c}\text { M. mysta- } \\
\text { cinus }\end{array}$ & N. noctula & $\begin{array}{c}\text { M. dau- } \\
\text { bentoni }\end{array}$ & P. auritus \\
\hline $\begin{array}{l}\text { Anzahl der } \\
\text { untersuchten }\end{array}$ & 4 & 15 & 5 & 6 & 4 & 1 \\
Exemplare & & & & & & \\
Blei & 19,5 & 13,5 & 8,40 & 8,03 & 4,16 & 2,13 \\
Cadmium & 0,317 & 0,212 & 0,125 & 0,272 & 0,260 & 0,745 \\
Chrom & 5,53 & 6,69 & 3,66 & 3,86 & 5,45 & 3,17 \\
Kupfer & 12,6 & 13,9 & 12,5 & 14,8 & 14,2 & 15,3 \\
Nickel & 4,98 & 3,87 & 5,82 & 2,21 & 2,52 & 0,73 \\
\hline \hline
\end{tabular}


Untersuchungen von Organ- und Gewebeproben an insgesamt zehn Tieren ergaben starke Schwankungen zwischen den gemessenen Konzentrationen für die Elemente Blei und Cadmium, die bis zum 100fachen zwischen dem ermittelten niedrigsten und dem höchsten Gehalt betrugen (Tab. 40, 41).

Die Untersuchungen von Organ- und Gewebeproben von sieben adulten und 14 juvenilen Zwergfledermäusen (Pipistrellus pipistrellus), die im Frühjahr 1985 gesammelt wurden, ergaben in adulten Tieren für Blei und Cadmium deutlich höhere Gehalte in allen untersuchten Organen als bei juvenilen Tieren (STREIT und NAGEL 1993b). Für die Elemente Chrom, Kupfer und Nickel war hingegen kein einheitlicher Trend erkennbar (Tab. 42).

Tab. 40: Ergebnisse der Schwermetalluntersuchungen an Organproben von Fledermaustotfunden aus Hessen (Deutschland). Die Zahl der untersuchten Tiere betrug 10 Individuen aus 6 Taxa. Nach StREIT und NAGEL (1993a). Alle Meßwerte in $\mu \mathrm{g} / \mathrm{g}$ TS

\begin{tabular}{|c|c|c|c|c|c|}
\hline Element & & Leber & Nieren & Lunge & Muskel \\
\hline Blei & $\begin{array}{l}\text { Min. } \\
\text { MW } \\
\text { Max. }\end{array}$ & $\begin{array}{r}0,9 \\
4,4 \\
38,5\end{array}$ & $\begin{array}{l}1,3 \\
2,8 \\
6,0\end{array}$ & $\begin{array}{l}0,4 \\
1,5 \\
3,9\end{array}$ & $\begin{array}{l}0,6 \\
1,6 \\
2,7\end{array}$ \\
\hline Cadmium & $\begin{array}{l}\text { Min. } \\
\text { MW } \\
\text { Max. }\end{array}$ & $\begin{array}{l}0,03 \\
0,21 \\
2,98\end{array}$ & $\begin{array}{l}0,04 \\
0,21 \\
3,24\end{array}$ & $\begin{array}{l}0,02 \\
0,11 \\
1,69\end{array}$ & $\begin{array}{l}0,01 \\
0,04 \\
0,46\end{array}$ \\
\hline Chrom & $\begin{array}{l}\text { Min. } \\
\text { MW } \\
\text { Max. }\end{array}$ & $\begin{array}{l}1,0 \\
2,0 \\
9,6\end{array}$ & $\begin{array}{l}0,6 \\
2,0 \\
5,0\end{array}$ & $\begin{array}{r}0,7 \\
2,5 \\
10,2\end{array}$ & $\begin{array}{l}0,7 \\
2,1 \\
6,4\end{array}$ \\
\hline $\begin{array}{l}\text { Kobalt } \\
\text { Kupfer }\end{array}$ & $\begin{array}{l}\text { Min. } \\
\text { MW } \\
\text { Max. } \\
\text { Min. } \\
\text { MW } \\
\text { Max. }\end{array}$ & $\begin{array}{r}0,2 \\
0,6 \\
1,7 \\
12,6 \\
25,9 \\
70,4\end{array}$ & $\begin{array}{r}0,3 \\
0,7 \\
1,8 \\
9,3 \\
20,2 \\
36,8\end{array}$ & $\begin{array}{r}0,1 \\
0,6 \\
2,4 \\
4,4 \\
9,9 \\
20,5\end{array}$ & $\begin{array}{r}0,2 \\
0,8 \\
2,5 \\
21,0 \\
31,0 \\
38,6\end{array}$ \\
\hline Nickel & $\begin{array}{l}\text { Min. } \\
\text { MW } \\
\text { Max. }\end{array}$ & $\begin{array}{l}0,4 \\
1,1 \\
3,0\end{array}$ & $\begin{array}{l}0,2 \\
1,4 \\
4,6\end{array}$ & $\begin{array}{l}0,4 \\
1,2 \\
3,8\end{array}$ & $\begin{array}{l}0,6 \\
1,6 \\
2,9\end{array}$ \\
\hline Zink & $\begin{array}{l}\text { Min. } \\
\text { MW } \\
\text { Max. }\end{array}$ & $\begin{array}{l}100 \\
165 \\
346\end{array}$ & $\begin{array}{l}116 \\
148 \\
308\end{array}$ & $\begin{array}{r}61 \\
95 \\
156\end{array}$ & $\begin{array}{r}50 \\
78 \\
129\end{array}$ \\
\hline Antimon & $\begin{array}{l}\text { Min. } \\
\text { MW } \\
\text { Max. }\end{array}$ & $\begin{array}{r}0,9 \\
<2,0 \\
4,5\end{array}$ & $\begin{array}{r}- \\
<0,7 \\
1,0\end{array}$ & $\begin{array}{l}- \\
- \\
-\end{array}$ & $\begin{array}{l}- \\
- \\
-\end{array}$ \\
\hline Selen & $\begin{array}{l}\text { Min. } \\
\text { MW } \\
\text { Max. }\end{array}$ & $\begin{array}{r}- \\
<1,5 \\
1,5\end{array}$ & $\begin{array}{r}- \\
<1,5 \\
1,8\end{array}$ & $\begin{array}{r}- \\
<1,2 \\
1,2\end{array}$ & $\begin{array}{l}- \\
-\end{array}$ \\
\hline
\end{tabular}

Min. $=$ niedrigster ermittelter Gehalt, MW = geom. Mittelwert, Max. $=$ höchster ermittelter Gehalt 
GeRELL \& GeRELL (1993) führten Untersuchungen von Leber und Nieren an insgesamt 32 Zwergfledermäusen durch. Die Messungen wurden mittels ICP-OES ausgeführt. Angaben zum Aufschlußverfahren fanden sich nicht. Die Autoren sprechen jedoch davon, daß sich die angegebenen Quecksilberwerte auf Quecksillber in Form von Methylquecksilber beziehen sollen. Eine Unterscheidung zwischen anorganischen und organischen Quecksilberverbindungen ist mittels ICP-OES analytisch jedoch nicht machbar.

Die untersuchten Tiere entstammten einer intensiv landwirtschaftlich genutzten Region um Alnarp, etwa $10 \mathrm{~km}$ südwestlich von Lund in Südschweden, sowie einem großen Nadelwaldgebiet um Öved, etwa $30 \mathrm{~km}$ östlich von Lund. Die ermittelten Cadmiumgehalte in Leber und Nieren zeigen bei Jungtieren eine niedrige Belastung und entsprechen in der Größenordnung denen von STREIT und NAGEL (1993b). Kein deutlicher Unterschied ist hingegen in der Belastung der untersuchten juvenilen und adulten Tiere durch Quecksilber zu erkennen (Tab. 43).

Tab. 41: Ergebnisse der Schwermetalluntersuchungen an Gewebeproben von Fledermaustotfunden aus Hessen (Deutschland). Die Zahl der untersuchten Tiere betrug 10 Individuen aus 6 Taxa. Nach STREIT und NAGEL (1993a). Alle Meßwerte in $\mu \mathrm{g} / \mathrm{g}$ TS

\begin{tabular}{|c|c|c|c|c|}
\hline Element & & $\begin{array}{c}\text { Knochen } \\
\text { (Femur) }\end{array}$ & Haare & Krallen \\
\hline Blei & $\begin{array}{l}\text { Min. } \\
\text { MW } \\
\text { Max. }\end{array}$ & $\begin{array}{r}1,5 \\
6,2 \\
34,8\end{array}$ & $\begin{array}{r}1,5 \\
4,1 \\
36,5\end{array}$ & $\begin{array}{r}1,5 \\
7,9 \\
36,5\end{array}$ \\
\hline Cadmium & $\begin{array}{l}\text { Min. } \\
\text { MW } \\
\text { Max. }\end{array}$ & $\begin{array}{l}0,01 \\
0,03 \\
0,23\end{array}$ & $\begin{array}{l}0,22 \\
0,93 \\
2,89\end{array}$ & $\begin{array}{l}0,10 \\
0,85 \\
3,81\end{array}$ \\
\hline Chrom & $\begin{array}{l}\text { Min. } \\
\text { MW } \\
\text { Max. }\end{array}$ & $\begin{array}{r}2,0 \\
4,7 \\
12,7\end{array}$ & $\begin{array}{r}1,5 \\
6,2 \\
15,1\end{array}$ & $\begin{array}{r}0,9 \\
8,3 \\
55,5\end{array}$ \\
\hline Kobalt & $\begin{array}{l}\text { Min. } \\
\text { MW } \\
\text { Max. }\end{array}$ & $\begin{array}{l}0,6 \\
1,1 \\
2,3\end{array}$ & $\begin{array}{l}0,2 \\
0,4 \\
0,6\end{array}$ & $\begin{array}{r}0,2 \\
<0,7 \\
3,0\end{array}$ \\
\hline Kupfer & $\begin{array}{l}\text { Min. } \\
\text { MW } \\
\text { Max. }\end{array}$ & $\begin{array}{l}1,6 \\
4,3 \\
7,8\end{array}$ & $\begin{array}{r}5,7 \\
23,1 \\
42,2\end{array}$ & $\begin{array}{r}7,7 \\
18,6 \\
42,6\end{array}$ \\
\hline Nickel & $\begin{array}{l}\text { Min. } \\
\text { MW } \\
\text { Max. }\end{array}$ & $\begin{array}{l}0,9 \\
3,3 \\
9,4\end{array}$ & $\begin{array}{l}0,8 \\
2,9 \\
8,1\end{array}$ & $\begin{array}{c}0,6 \\
4,1 \\
59\end{array}$ \\
\hline Zink & $\begin{array}{l}\text { Min. } \\
\text { MW } \\
\text { Max. }\end{array}$ & $\begin{array}{r}59 \\
100 \\
141\end{array}$ & $\begin{array}{l}105 \\
356 \\
610\end{array}$ & $\begin{array}{r}48 \\
63 \\
100 \\
\end{array}$ \\
\hline
\end{tabular}

Min. = niedrigster ermittelter Gehalt, MW = geom. Mittelwert, Max. = höchster ermittelter Gehalt 
Tab. 42: Ergebnisse der Schwermetalluntersuchungen an Organproben aus Totfunden von Pipistrellus pipistrellus im Raum Darmstadt (Hessen, Deutschland) aus dem Frühjahr 1985. Die Daten basieren auf der Messung von 14 adulten (ad.) und 7 juvenilen (juv.) Tieren. Nach StREIT und NAGEL (1993b). Alle Meßwerte in $\mu \mathrm{g} / \mathrm{g}$ TS

\begin{tabular}{lllll}
\hline \hline \multicolumn{1}{c}{ Element } & & Leber & Nieren & Lunge \\
\hline Blei & ad. & 2,95 & 5,90 & 5,83 \\
\multirow{2}{*}{ Cadmium } & juv. & 1,94 & 2,45 & 1,12 \\
\multirow{2}{*}{ Chrom } & ad. & 1,530 & 5,133 & 0,288 \\
& juv. & 0,062 & 0,088 & 0,074 \\
\multirow{2}{*}{ Kupfer } & ad. & 2,9 & 6,1 & 11,3 \\
& juv. & 4,6 & 12,6 & 6,6 \\
\multirow{2}{*}{ Nickel } & ad. & 15,7 & 22,7 & 6,8 \\
& juv. & 15,2 & 15,5 & 8,1 \\
\hline \hline
\end{tabular}

Tab. 43: Cadmium und (Methyl)-Quecksilbergehalte in Leber und Nieren unterschiedlich alter Zwergfledermäuse ( $P$. pipistrellus) aus zwei Regionen östlich von Lund, Südschweden. Die Tiere wurden im Jahr 1984 gesammelt. Nach GereLl und GereLL (1993). Alle Meßwerte in $\mu \mathrm{g} / \mathrm{g}$ TS

\begin{tabular}{cccccc}
\hline \hline \multirow{2}{*}{ Herkunft } & \multicolumn{2}{c}{ Cadmium } & \multicolumn{2}{c}{ Quecksilber } \\
& Alter & Leber & Nieren & Leber & Niere \\
& (Monate) & & & & \\
\hline Alnarp & $>14$ & 3,3 & 4,4 & 3,2 & 2,3 \\
Öved & $>12$ & 0,7 & 2,1 & 2,9 & 4,7 \\
Öved & 1 & n.n. & n.n. & 3,6 & 1,9 \\
\hline \hline
\end{tabular}

n.n. = nicht nachweisbar

ZYRNICKI et al. (1996) führten an Haarproben von zwei Großen Mausohren (Myotis myotis) Untersuchungen des Elementgehaltes mittels ICP-OES durch. Der Probenaufschluß erfolgte mit Salpetersäure mittels Mikrowelle. Weitere Angaben zur Probenvorbehandlung wie z.B. Waschen der Haare fanden sich nicht. Die untersuchten Tiere entstammten einer künstlichen Höhle im Bereich des Swietokrzyskiegebirges in Zentralpolen, zwischen Warschau und Krakau, wo sie im Februar 1995 gesammelt wurden. An den publizierten Daten fällt auf, daß der Aluminiumgehalt deutlich höher liegt als der ermittelte Zinkgehalt (Tab. 44). Im Vergleich zu anderen Haaruntersuchungen von Säugetieren liegt bei den untersuchten Haarproben vom Großen Mausohr der Aluminiumgehalt etwa um das 10fache höher (KATZ und CHATT 1988; KIJEWSKI 1993). Der Zinkgehalt ist hingegen um den Faktor 2 bis 3 niedriger als bei vergleichbaren Säugetierdaten. Weitere Untersuchungen zum Schwermetallgehalt in 
Fledermäusen sind am Centrum Informacji Chiropterologicznej des Instituts für Systematik und Evolution der Tiere der Polnischen Akademie der Wissenschaft für das Jahr 2000 und 2001 vorgesehen (WOLOSZYN et al. 1999, WOLOSZYN Schr. Mitt.).

Tab. 44: Elementgehalte in Haarproben vom Großen Mausohr (M. myotis) aus einem Gebiet im Swietokrzyskiegebirge in Zentralpolen. Die Tiere wurden im Februar 1995 gesammelt. ZYRNICKI et al. (1996)

\begin{tabular}{llcc}
\hline \hline & Einheit & Tier 1 & Tier 2 \\
\hline Probeneinwaage & {$[\mathrm{mg}]$} & 10,7 & 15,5 \\
Aluminium & {$[\mu \mathrm{g} / \mathrm{g}$ TS] } & 240 & 120 \\
Barium & {$[\mu \mathrm{g} / \mathrm{g}$ TS] } & 136 & 35 \\
Calcium & {$[\mu \mathrm{g} / \mathrm{g}$ TS $]$} & 30 & 2850 \\
Kupfer & {$[\mu \mathrm{g} / \mathrm{g}$ TS $]$} & 114 & 73 \\
Eisen & {$[\mu \mathrm{g} / \mathrm{g}$ TS $]$} & 38 & 250 \\
Magnesium & {$[\mu \mathrm{g} / \mathrm{g}$ TS $]$} & 9 & 114 \\
Mangan & {$[\mu \mathrm{g} / \mathrm{g}$ TS] } & 17 & 14 \\
Silizium & {$[\mu \mathrm{g} / \mathrm{g}$ TS $]$} & 710 & 570 \\
Zink & {$[\mu \mathrm{g} / \mathrm{g}$ TS] } & 124 & 116 \\
\hline \hline
\end{tabular}

In der internationalen Literatur wurden u.a. Vergleichsuntersuchungen zwischen der Schwermetallbelastung von Fledermäusen und anderen Kleinsäugern aus einer Region unweit einer stark befahrenen Hauptverkehrsstraße von CLARK (1979) publiziert. Die Untersuchungen wurden am Ganztieraufschluß nach dem Entfernen von Darm und Embryonen durchgeführt. Die Tiere wurden zunächst homogenisiert und anschließend einem Naßaufschluß mit Salpetersäure unterworfen. Die Messungen erfolgten mittels AAS. Die Untersuchungen ergaben für Fledermäuse signifikant höhere Bleigehalte als für andere untersuchte Kleinsäuger. Die Bleibelastung der Männchen der Großen Braunen Fledermaus (Eptesicus fuscus) lag dabei signifikant höher als die der Weibchen (Tab. 45).

Tab. 45: Bleigehalte in Ganztieraufschlüssen von Eptesicus fuscus und Myotis lucifugus aus Montpelier Barn (USA). Probenahmedatum Mai/Juni 1976. Nach CLARK (1979)

\begin{tabular}{lcccc}
\hline \hline & Einheit & $\begin{array}{c}\text { Eptesicus } \\
\text { fuscus }\end{array}$ & $\begin{array}{c}\text { Eptesicus } \\
\text { fuscus }\end{array}$ & $\begin{array}{c}\text { Myotis } \\
\text { lucifugus }\end{array}$ \\
\hline Geschlecht & $\mathrm{m}$ & $\mathrm{w}$ & $\mathrm{w}$ \\
Anzahl der untersuchten & & 8 & 10 & 12 \\
$\quad$ Exemplare & & 46,55 & 31,49 & 16,97 \\
Blei, geom. Mittelwert & {$[\mu \mathrm{g} / \mathrm{g}$ TS] } & $20-90$ & $20-56$ & $11-29$ \\
Blei, Schwankungsbreite & {$[\mu \mathrm{g} / \mathrm{g}$ TS] } & &
\end{tabular}


Nachfolgende Untersuchungen von CLARK et al. (1986) wurden an Fledermauspopulationen von Myotis austroriparius zur Belastung durch Blei, Chrom, Cadmium und Zink durchgeführt. Verglichen wurden dabei Populationen aus einer Höhle (Judges Cave) unweit eines durch Abfälle einer Batteriefabrik in Steel City (Jackson Country, Florida, USA) kontaminierten Gewässers mit einer unweit einer Autobahnbrücke lebenden Population bei Gainesville. Der Aufschluß der Gewebeproben erfolgte mit $1 \mathrm{ml}$ Salpetersäure in Polypropylengefäßen. Die Analysen wurden mittels Flammenund Graphitrohr-AAS ausgeführt. Die Gehalte an Zink im Lebergewebe lagen in den untersuchten Tieren aus dem Umfeld der Batterieproduktion geringfügig, aber signifikant über denen der Vergleichspopulation bei Gainesville. Die Cadmiumgehalte in der Leber und in den Nieren der Tiere aus der Judges Cave lagen 2- bis 3-fach über denen der Vergleichspopulation (Tab. 46). Allerdings handelte es sich bei den untersuchten Tieren aus der Judges Cave zum überwiegenden Teil um Weibchen ( 9 der 10 untersuchten Tiere), wohingegen die untersuchten zehn Tiere aus Gainesville ausnahmslos Männchen waren. CLARK et al. kamen zu dem Ergebnis, daß die höchste ermittelte Cadmiumkonzentration im Nierengewebe $(2,9 \mu \mathrm{g} / \mathrm{g} \mathrm{FS})$ noch deutlich unter der Minimumkonzentration der Schädigung von anderen Kleinsäugern liegt $(3,41,8,5$ und $44 \mu \mathrm{g} / \mathrm{g} \mathrm{FS})$.

Tab. 46: Schwermetallgehalte in Gewebeproben von Myotis austroriparius aus der Judges Cave (Florida, USA) im Umfeld einer Batterieproduktion und aus einem Quartier unweit einer Autobahnbrücke bei Gainesville (Florida, USA). Nach CLARK et al. (1986); alle Meßwerte in $\mu \mathrm{g} / \mathrm{g}$ FS

\begin{tabular}{lccc}
\hline \hline & Zink & Leber & Nieren \\
& & Cadmium & \begin{tabular}{c} 
Cadmium \\
\hline a) Judges Cave
\end{tabular} \\
\hline Anzahl unters. Tiere & 10 & 10 & 10 \\
Arithm. Mittelwert: & 31,1 & 0,63 & 1,13 \\
Geom. Mittelwert: & 30,5 & 0,612 & 0,889 \\
Spannweite: & $27-35$ & $0,36-0,85$ & $0,22-2,9$ \\
& & & \\
b) Gainesville & 10 & 10 & 10 \\
Anzahl unters. Tiere: & 28,5 & 0,30 & 0,406 \\
Arithm. Mittelwert: & 28,5 & 0,256 & 0,266 \\
Geom. Mittelwert: & $26-30$ & $0,14-0,75$ & $0,15-2,1$ \\
Spannweite: & &
\end{tabular}

THIES und GREGORY (1994) analysierten den Blei-, Cadmium- und Arsengehalt in der Leber der Brasilianischen Bulldoggfledermaus (Tadarida brasiliensis). Die Tiere stammten aus der Carlsbadhöhle, New Mexico, und der Vickeryhöhle nordwestlich von Oklahoma (USA), wo sie im Mai und im August 1991 gesammelt wurden. Das entnommene Lebergewebe wurde zur Bestimmung des Blei- und des Cadmiumgehaltes einem Naßaufschluß mit Salpetersäure bei $60^{\circ} \mathrm{C}$ für $24 \mathrm{~h}$ unterworfen. Für die 
Arsenbestimmung erfolgte der Aufschluß mit Salpetersäure unter Zugabe von Nikkelnitrat. Die Analyse von Blei, Cadmium und Arsen erfolgte mittels Graphitrohr-AAS.

Die Analysen ergaben für Arsen lediglich bei einem untersuchten Tier einen meßbaren Gehalt von 0,353 $\mu \mathrm{g} / \mathrm{g}$ FS. Blei konnte in allen untersuchten 48 Tieren nachgewiesen werden, Cadmium in 44 der untersuchten Tiere. Die Bestimmungsgrenze betrug bei diesen Untersuchungen für alle Elemente jeweils 0,2 $\mu \mathrm{g} / \mathrm{g} \mathrm{FS}$.

Die von ThIES und GREGORY (1994) ermittelten Blei- und Cadmiumgehalte in der Leber von Tadarida brasiliensis liegen im Vergleich zu den Untersuchungen von STREIT und NAGEL (1993b) an mitteleuropäischen Microchiropteren deutlich höher (Tab. 47). Beim direkten Vergleich der Meßwerte der beiden Autorengruppen ist zudem zu berücksichtigen, daß THIES und GREGORY ihre Daten auf die Feuchtmasse beziehen, STREIT und NAGEL hingegen auf die Trockenmasse. Eigene Untersuchungen ergaben für Lebergewebeproben einen mittleren Feuchtegehalt von 73 Gew.-\%.

Tab. 47: Geometrisches Mittel $(n=6)$ und Schwankungsbreite (in Klammern) der Blei- und Cadmiumgehalte im Lebergewebe der Brasilianischen Bulldoggfledermaus (Tardarida brasiliensis) aus zwei Höhlen im südlichen Nordamerika. Nach THIES und GREGORY (1994). Alle Meßwerte in $\mu \mathrm{g} / \mathrm{g}$ FS

a) Blei:

\begin{tabular}{cccc}
\hline \hline Herkunft & Geschlecht & Mai 1991 & August 1991 \\
\hline Carlsbad Höhle & w & $1,91(1,41-2,96)$ & $4,35(1,11-13-70)$ \\
Vickery Höhle & m & $3,51(1,25-7,72)$ & $3,67(1,45-16,00)$ \\
& w & $2,22(0,74-7,07)$ & $5,07(2,47-34,90)$ \\
& m & $5,37(1,84-49,44)$ & $5,34(1,40-27,64)$ \\
\hline \hline
\end{tabular}

a) Cadmium:

\begin{tabular}{cccc}
\hline \hline Herkunft & Geschlecht & Mai 1991 & August 1991 \\
\hline Carlsbad Höhle & $\mathrm{w}$ & $0,53(0,22-1,31)$ & $0,80(0,52-1,55)$ \\
Vickery Höhle & $\mathrm{m}$ & $0,44(0,28-0,67)$ & $0,88(0,51-1,46)$ \\
& $\mathrm{w}$ & $0,88(0,47-1,52)$ & $1,16(0,60-1,98)$ \\
& $\mathrm{m}$ & $0,44(<0,20-1,02)$ & $0,20(<0,20-0,22)$ \\
\hline \hline
\end{tabular}

Die Untersuchung von sechs Pipistrellus subflavus aus dem Bereich des stark quecksilberbelasteten Holston-River (West-Virginia, USA) unterhalb einer ChlorAlkali-Elektrolyse ergab im Jahr 1978 mittlere Quecksilbergehalte im Muskelgewebe von $0,86 \mu \mathrm{g} / \mathrm{g}$ und im Lebergewebe von 1,92 $\mu \mathrm{g} / \mathrm{g}$ (G.V.N. POWELL, zit. nach CLARK 1981).

Untersuchungen von CLARK et al. (1986) an Myotis austroriparius aus Florida ergaben maximale Leberbleiwerte von $0,58 \mu \mathrm{g} / \mathrm{g}$. 
REIDINGER (1972) untersuchte die Quecksilberbelastung in der Atemmuskulatur von fünf verschiedenen Fledermausarten aus dem südöstlichen Arizona (USA) und dem angrenzenden Mexico. Die ermittelten mittleren Quecksilberbelastungen der einzelnen Arten variieren zwischen $0,006 \mu \mathrm{g} / \mathrm{g}$ und $0,22 \mu \mathrm{g} / \mathrm{g}$ (Tab. 48).

Tab. 48: Quecksilbergehalte in der Atemmuskulatur verschiedener Fledermausarten aus dem südöstlichen Arizona (USA) und dem angrenzenden Mexico. Nach REIDINGER (1972)

\begin{tabular}{lcc}
\hline \hline \multicolumn{1}{c}{ Art } & $\begin{array}{c}\text { Anzahl der } \\
\text { unters. Tiere }\end{array}$ & $\begin{array}{c}\text { mittl. Quecksilbergehalt } \\
{[\boldsymbol{\mu g} / \mathbf{g}]}\end{array}$ \\
\hline Eptesicus fuscus & 5 & 0,218 \\
Pipistrellus hesperus & 2 & 0,13 \\
Tardarida brasiliensis & 5 & 0,074 \\
Anthrozous pallidus & 5 & 0,03 \\
Leptonycteris sanborni & 4 & 0,0065 \\
\hline \hline
\end{tabular}

MIURA et al. (1978) untersuchten Organ- und Gewebeproben sowie Haare auf Quecksilber an Museumstieren (Alkoholpräparate) aus dem Jahr 1890 sowie an Tierfunden aus den Jahren 1965-1967 und 1970-1975. Die Messungen erfolgten mittels Atomabsorption. Im Untersuchungszeitraum 1965-1967 erfolgte in Japan die Verwendung von quecksilberhaltigen Fungiziden. Im Vergleich zu dem Probenmaterial aus dem Jahr 1890 lag der Quecksilbergehalt Mitte des 20. Jahrhunderts in den untersuchten Proben etwa 4- bis 6fach höher (Tab. 49). Die bevorzugten Anreicherungsgewebe für Quecksilber (Leber und Nieren) wiesen Gehalte bis zu 1,15 $\mu \mathrm{g} / \mathrm{g}$ auf.

Tab. 49: Schwankungsbreite der Quecksilbergehalte in drei verschiedenen Fledermausarten (Rhinolophus cornutus cornutus, Rhinolophus ferrum-equinum nippon, Miniopterus schreibersi fuliginosus) aus dem südlichen Japan. Alle Meßwerte in $\mu \mathrm{g} / \mathrm{g}$. Nach MiURA et al. (1978)

\begin{tabular}{lcccccc}
\hline \hline Fundzeitraum & $\begin{array}{c}\text { Anz. unters. } \\
\text { Ex. }\end{array}$ & Niere & Leber & Gehirn & Muskel & Haare \\
\hline 1890 & 6 & 0,11 & 0,19 & - & 0,25 & 2,63 \\
$1965-1967$ & 46 & $0,84-0,99$ & $0,69-1,15$ & $0,10-0,35$ & $0,19-0,64$ & $3,49-10,5$ \\
$1970-1975$ & 18 & $0,51-0,63$ & $0,35-0,87$ & $0,05-0,25$ & $0,08-0,42$ & $6,34-10,2$ \\
\hline \hline
\end{tabular}

Im Rahmen einer Studie zum Selengehalt im menschlichen Gewebe untersuchten SCHROEDER et al. (1970) zu Vergleichszwecken einige andere Säugetiere. Unter anderem wurde auch eine Gruppe nicht näher determinierter Fledermäuse (drei Exemplare) hinsichtlich der Selenverteilung im Gewebe untersucht (Tab. 50). Die Autoren kamen zu dem Ergebnis, daß für die untersuchten Säuger der Selengehalt in 
Herz, Lunge und Milz (ohne Fledermäuse) annähernd gleich dem der Humangewebe war. Für das Leber- und Nierengewebe lagen die Selengehalte hingegen deutlich oberhalb der Werte von Humangewebe.

Tab. 50: Mittlere Selengehalte in Gewebeproben von Fledermäusen $(n=3)$, nach SCHROEDER et al. (1970)

\begin{tabular}{|c|c|c|}
\hline Gewebe & $\begin{array}{l}\text { Selengehalt [ } \mu \mathrm{g} / \mathrm{g} \text { FS] } \\
\text { in Fledermäusen }\end{array}$ & $\begin{array}{c}\text { Selengehalt }[\mu \mathrm{g} / \mathrm{g} \mathrm{FS}] \\
\text { Mittel aller untersuchten } \\
\text { Säuger }\end{array}$ \\
\hline$\overline{\text { Herz }}$ & 2,86 & 0,75 \\
\hline Lunge & 0,77 & 0,62 \\
\hline Leber & 1,07 & 1,26 \\
\hline Niere & 1,17 & 2,16 \\
\hline Flügel und Füße & 3,87 & - \\
\hline Haut und Haare & 4,0 & - \\
\hline
\end{tabular}

Alvarez-CASTANEDA et al. (1998) untersuchten Myotis vivesi und Noctilio leporinus aus Mexico. In $M$. vivesi lagen die mittels Atomabsorbtionsspektralphotometrie ermittelten Gehalte für Blei, Eisen, Kupfer, Magnesium und Zink deutlich über denen von Noctilio leporinus. Kein signifikanter Unterschied zeigte sich hingegen beim Cadmium.

\subsubsection{Megachiroptera}

Zur Schwermetallbelastung von Flederhunden (Megachiroptera) finden sich in der Literatur Angaben bei ZOOK et al. (1970), SUTTON und WILSON (1983), SUTTON und HARIONO (1986) und SKERRATT et al. (1998).

Die beiden von Zook et al. untersuchten Totfunde der Art Pteropus poliocephalus ernähren sich ausschließlich von Früchten. Im Lebergewebe fanden sich Bleigehalte von mehr als $500 \mu \mathrm{g} / \mathrm{g}$ FS. Ursache für diese hohe Bleibelastung war der Verzehr von bleihaltiger Farbe durch die im Zoo von Washington (USA) lebenden Tiere. ZooK et al. beschreiben darüber hinaus, daß im Cortexgewebe intranucleare Einschlüsse im mikroskopischen Bild erkennbar waren, die typisch für Bleivergiftungen sind. Hierbei handelt es sich um Blei-Proteinablagerungen im Zellkern. Ähnliche Gewebeveränderungen beschreiben SUTTON und WILSON (1983) an zwei Exemplaren von Pteropus poliocephalus, die im Raum Brisbane (Australien) gefunden wurden. Im Nieren- und Lebergewebe wurden im Vergleich zur Untersuchung von ZooK jedoch deutlich geringere Bleigehalte nachgewiesen (Tab. 51). Ein direkter Vergleich der Meßwerte ist jedoch aufgrund fehlender Angabe der Bezugssubstanz (Trockenbzw. Feuchtmasse) nicht möglich. 
Tab. 51: Bleigehalte in Gewebeproben von Pteropus poliocephalus aus Brisbane (Australien). Beide Tiere zeigten auffällige intranucleare Ablagerungen, wie sie für Bleivergiftungen typisch sind. Nach SUTTON und WILSON (1983)

\begin{tabular}{cccc}
\hline \hline & Einheit & Nieren & Leber \\
\hline Tier A & {$[\mu \mathrm{g} / \mathrm{g}]$} & 20,5 & 59,5 \\
Tier B & {$[\mu \mathrm{g} / \mathrm{g}]$} & 44,6 & 18,7 \\
\hline \hline
\end{tabular}

In einer umfangreicheren Studie untersuchten SUTTON und HARIONO (1986) insgesamt 49 fruchtfressende Flughunde fünf verschiedener Arten (Pteropus poliocephalus, P. scapulatus, P. alecto, P. conspicillatus, Dobsonia moluccensis). Die Tiere stammten überwiegend aus der Region um Brisbane (Australien) und wiesen z.T. Vergiftungserscheinungen auf. Bei allen untersuchten Tieren handelte es sich um Totfunde oder Tiere, die aufgrund von Verhaltensauffälligkeiten gefangen wurden und kurz nach dem Fang starben. Die Messungen wurden mittels AAS ausgeführt.

Die Tiere mit erkennbaren Vergiftungserscheinungen (unkoordinierte Bewegungsabläufe, Muskelzittern, Appetitlosigkeit) wiesen deutliche Bleigehalte im Leber- und im Nierengewebe auf (Tab. 52). Die Bleigehalte lagen jedoch innerhalb der Vergleichsgruppe aus dem stadtnahen Umfeld von Brisbane. Die aus dem Umfeld von Brisbane stammenden Tiere weisen im Vergleich zu Totfunden aus naturnahen, stadtfernen Gebieten (Forest Hill und Cape York) deutlich höhere Maximalwerte für Blei im Leber- und Nierengewebe auf.

Tab. 52: Bleigehalte in Leber und Nieren von australischen Flughunden. Nach SUTTON und HARIONO (1986); alle Meßwerte in $\mu \mathrm{g} / \mathrm{g}$ FS

\begin{tabular}{lccc}
\hline \hline \multicolumn{1}{c}{ Herkunft } & Anz. unters. Ex. & Leber & Nieren \\
\hline Brisbane, mit Vergiftungser- & 3 & $12,1-47,4$ & $21,7-30,8$ \\
scheinungen & 27 & $0,3-39,5$ & $0,2-65,3$ \\
Brisbane & 7 & $2,8-10,9$ & $3,1-7,9$ \\
Forest Hill & 1 & 1,0 & 0,6 \\
Cape York & & \\
\hline \hline
\end{tabular}

SKERRATT et al. (1998) untersuchten vier Exemplare der Art Pteropus alecto aus Queensland (Australien). Den Angaben ist jedoch nicht zu entnehmen, ob es sich hierbei um die Trockensubstanz als Bezugsgröße oder um das Feuchtmaterial handelt. Bei zwei Exemplaren wurde eine Lyssavirusinfektion festgestellt. Eines der infizierten Tiere wies zudem eine hohe Bleibelastung auf (Tab. 53). Das Verhalten von lyssavirusinfizierten Tieren ähnelt dabei dem Verhalten von bleivergifteten Tieren, wie sie von SUTTON und WILSON (1983) beschrieben wurden. Anhand von Verhaltensauffälligkeiten ist somit keine gesicherte Aussage über eine Virusinfektion des Tieres möglich. 
Tab. 53: Blei- und Cadmiumgehalte in Gewebeproben von Pteropus alecto aus Queensland (Australien). Nach SKERRATT et al. (1998). Meßwerte in $\mu \mathrm{g} / \mathrm{g}$

\begin{tabular}{|c|c|c|c|c|c|c|}
\hline \multirow[b]{2}{*}{ Tier Nr. } & \multirow[b]{2}{*}{ Nieren } & \multirow{2}{*}{$\begin{array}{l}\text { Blei- } \\
\text { Leber }\end{array}$} & \multirow[b]{2}{*}{ Gehirn } & \multirow[b]{2}{*}{ Nieren } & Cadmium & \multirow[b]{2}{*}{ Gehirn } \\
\hline & & & & & Leber & \\
\hline 1 & 370,03 & 16,76 & 2,32 & 8,09 & 1,36 & $<0,03$ \\
\hline 2 & 123,85 & 19,26 & $<0,6$ & 0,16 & $<0,03$ & $<0,03$ \\
\hline 3 & n.b. & 1,54 & n.b. & n.b. & 0,2 & n.b. \\
\hline 4 & 2,29 & $<0,6$ & $<0,6$ & 1,79 & 0,17 & $<0,03$ \\
\hline
\end{tabular}

n.b. = nicht bestimmt

\subsubsection{Schwermetallgehalte im Fledermauskot}

Im Vergleich zu Untersuchungen von Organ- und Gewebeproben aus Fledermäusen lassen sich Kotproben wesentlich einfacher gewinnen, aufarbeiten und analysieren. Zudem sind hier in der Regel ausreichende Probenmengen verfügbar. Die Untersuchung von Fledermauskot gestattet einen ersten Rückschluß auf die oral über die Nahrung aufgenommene Schwermetallmenge. Durch Kotanalysen können, bei Vorliegen ausreichender Vergleichsdaten, somit rasch erhöhte Schwermetallaufnahmen erkannt werden, wie sie z.B. nach Dachstuhlmodernisierungen mit frisch imprägnierten Hölzern auf der Basis von chrom- oder kupfersalzhaltigen Imprägniermitteln (sog. CKB oder CKA-Salze) möglich sind. Je nach Fundzustand besteht, besonders bei der Beprobung geringer Kotmengen, im Vergleich zu den zuvor beschriebenen Gewebe- und Haaranalysen eine größere Gefahr der Kontamination der Probenmaterials, z.B. durch anhaftenden Staub oder Boden.

Die Datenlage zum Schwermetallgehalt von Fledermauskot aus Mitteleuropa ist gegenwärtig sehr gering und beschränkt sich auf stichprobenartige Untersuchungen (ULOTH et al. 1987, SCHÄFFLER 1989 und RACKOW 1991). Darüber hinaus liegen vereinzelt Daten aus Quartieren in Arizona (PETIT und ALTENBACH 1973), Florida (CLARK et al. 1986) und Kentucky (LACKI 1994) vor.

ULOTH et al. (1987) untersuchten an einem Bohrkern aus einem etwa 20 Jahre alten Kothaufen einer Wochenstube des Großen Mausohres (Myotis myotis) bei Neidhartshausen/Rhön (Südthüringen) den Gehalt an Blei und Cadmium aus unterschiedlichen Horizonten. Angaben zum Untersuchungsverfahren finden sich nicht. Die mittlere Bleikonzentration betrug $11,8 \mathrm{mg} / \mathrm{kg}$ TS, die mittlere Cadmiumkonzentration $5,7 \mathrm{mg} / \mathrm{kg}$ TS. Während für Cadmium eine annähernde Gleichverteilung der Gehalte über den gesamten Bohrkern festzustellen war, lag für Blei die Konzentration in den oberen rund $10 \mathrm{~cm}$ des Kothaufens, also in den jüngeren Schichten, etwa um 50 $\%$ niedriger als in den unteren rund $15 \mathrm{~cm}$ des Kothaufens (Tab. 54). ULOTH et al. (1987) konstruieren hieraus, daß es beim Blei im Gegensatz zum Cadmium zu einer Tiefenverlagerung kommt. Welcher Prozeß eine solche Tiefenverlagerung des Bleis bewirken soll, beschreiben die Autoren nicht. Die Möglichkeit, daß anthropogen 
bedingte Emissionen im Umfeld des Quartieres in früherer Zeit zu erhöhten Bleidepositionen im Jagdhabitat der Mausohrkolonie geführt haben könnte, wurden nicht in Betracht gezogen.

Tab. 54: Gehalte von Blei und Cadmium in einem etwa 20 Jahre alten Kothaufen in einer Wochenstube des Großen Mausohres (M. myotis) bei Neidhartshausen/Rhön (Südthüringen). Nach ULOTH et al. (1987)

\begin{tabular}{cccc}
\hline \hline Schicht-Nr. & $\begin{array}{c}\text { ungefähre Lage des } \\
\text { Probenhorizontes unter } \\
\text { Haufenoberkante [cm] }\end{array}$ & $\begin{array}{c}\text { Blei } \\
\text { [mg/kg TS] }\end{array}$ & $\begin{array}{c}\text { Cadmium } \\
\text { [mg/kg TS] }\end{array}$ \\
\hline 1 & 2,5 & 5,0 & 5,8 \\
2 & 5,0 & 6,0 & 6,6 \\
3 & 7,5 & 7,3 & 5,7 \\
4 & 10,0 & 6,2 & 8,9 \\
5 & 12,5 & 13 & 6,4 \\
6 & 15,0 & 16 & 5,2 \\
7 & 17,5 & 12 & 3,7 \\
8 & 20,0 & 14 & 4,0 \\
9 & 22,5 & k.A. & k.A. \\
10 & 25,0 & 27 & 5,1 \\
\hline arithm. Mittelwert & \multicolumn{3}{|}{} \\
\hline \hline k.A. = keine Angabe & \multicolumn{3}{c}{}
\end{tabular}

Eine ähnliche Untersuchung führte RACKOW (1991) an zwei Bohrkernen aus einem Kothaufen einer Wochenstube des Großen Mausohres (Myotis myotis) bei Hattorf, Landkreis Osterode am Harz, sowie bei Northeim (beide Südniedersachsen) durch. Allerdings fehlen hier Angaben zur Bezugsgröße (Trockensubstanz bzw. Feuchtsubstanz). Die ermittelten Cadmiumgehalte lagen in ähnlicher Größenordnung wie in der Untersuchung von ULOTH et al. (1987). Beim Bleigehalt zeigten sich hingegen deutliche Unterschiede zwischen den Proben aus dem Quartier in Hattorf/Landkreis Osterode am Harz und dem Quartier bei Northeim. So lag der Bleigehalt in allen untersuchten Horizonten aus dem Quartier bei Hattorf etwa fünffach über den Werten aus Northeim. Eine signifikante Zunahme der Bleigehalte zu tieferen Lagen hin konnte nicht nachgewiesen werden (Tab. 55). Deutlich niedrigere Blei- und Cadmiumgehalte fand RACKOW (1991) in zwei Kotproben aus Mausohrwochenstuben in den ländlich geprägten Bereichen um Ershausen und Deuna im Landkreis Heiligenstadt/Thüringen. Hier lag der Bleigehalt zwischen $<1,0 \mathrm{mg} / \mathrm{kg}$ (Ershausen) und 2,2 $\mathrm{mg} / \mathrm{kg}$ (Deuna). Die ermittelten Cadmiumgehalte betrugen in beiden Stichproben 1,3 $\mathrm{mg} / \mathrm{kg}$. Die eigenen Untersuchungen des Verfassers an Kotproben aus dem selben Quartier in Hattorf führten für Blei zu vergleichbaren Ergebnissen (Kotproben 21 und 22, vgl. Anhang 7). Aufgrund der guten Übereinstimmung zu den eigenen Werten kann davon ausgegangen werden, daß die Meßwerte bei RACKOw sich auf Trockensubstanzangaben beziehen. 
Bei den von Rackow publizierten Daten aus dem Quartier in Northeim fällt das ungewöhnliche Blei/Cadmium-Verhältnis auf. So war der Cadmiumgehalt im Kot statistisch stark signifikant $(p<0,0001)$ höher als der Bleigehalt, ein Verhältnis, das aufgrund des geogenen Verteilungsverhältnisses höchst ungewöhnlich ist! Die eigenen Untersuchungen von Kotproben aus diesem Quartier ergaben lediglich halb so hohe Cadmiumgehalte.

Im Gegensatz zu den aus den achtziger Jahren des 20. Jahrhunderts stammenden Kotproben aus dem Quartier bei Hattorf konnte bei den horizontiert entnommenen Kotproben aus den Quartieren bei Ahlden und Steinbergen ein Rückgang des Bleigehaltes um rund $50 \%$ festgestellt werden. Alle übrigen Schwermetalle lagen in annähernd unveränderter Konzentration vor. Der Grund für den deutlichen Rückgang der Bleigehalte im Kot aus diesen beiden Quartieren dürfte in dem größeren zeitlichen Abstand von der Verwendung verbleiter Vergaserkraftstoffe zu sehen sein. Während die Proben aus dem Quartier in Hattorf und die Proben von Rackow aus dem Quartier in Northeim aus der Übergangsphase von verbleiten zu bleifreien Vergaserkraftstoffen stammen, deckten die im Rahmen dieser Arbeit untersuchten Proben aus Ahlden und Steinbergen den hieran anschließenden Zeitraum ab.

Tab. 55: Gehalte von Blei und Cadmium in mehrjährigen Kothaufen aus zwei Wochenstube des Großen Mausohres (M. myotis) bei Northeim und Hattorf/Harz (Südniedersachsen). Nach RACKOW (1991)

\begin{tabular}{|c|c|c|c|c|c|}
\hline \multirow[b]{2}{*}{$\begin{array}{c}\text { Schicht- } \\
\text { Nr. }\end{array}$} & \multirow[b]{2}{*}{$\begin{array}{c}\text { ungefähre Lage } \\
\text { des Proben- } \\
\text { horizontes unter } \\
\text { Haufenoberkante } \\
\text { [cm] }\end{array}$} & \multicolumn{2}{|c|}{ Hattorf/Harz } & \multicolumn{2}{|c|}{ Northeim } \\
\hline & & $\begin{array}{c}\text { Blei } \\
{[\mathrm{mg} / \mathrm{kg}]}\end{array}$ & $\begin{array}{l}\text { Cadmium } \\
\text { [mg/kg] }\end{array}$ & $\begin{array}{c}\text { Blei } \\
{[\mathrm{mg} / \mathrm{kg}]}\end{array}$ & $\underset{[\mathrm{mg} / \mathrm{kg}]}{\text { Cadmium }}$ \\
\hline 1 & 5 & 16,41 & 6,23 & 2,85 & 3,81 \\
\hline 2 & 10 & 15,89 & 6,04 & 3,61 & 4,40 \\
\hline 3 & 15 & 15,46 & 5,92 & 2,11 & 4,27 \\
\hline 4 & 20 & 15,08 & 7,24 & 2,55 & 3,75 \\
\hline 5 & 25 & 15,46 & 7,34 & 3,62 & 4,66 \\
\hline 6 & 30 & 15,27 & 6,74 & 3,68 & 4,25 \\
\hline 7 & 35 & 14,51 & 5,57 & 3,77 & 4,68 \\
\hline 8 & 40 & 16,37 & 6,27 & 3,42 & 4,07 \\
\hline 9 & 45 & 18,13 & 8,24 & 3,42 & 5,38 \\
\hline 10 & 50 & 18,41 & 8,43 & 3,45 & 5,79 \\
\hline 11 & 55 & - & - & 2,64 & 5,69 \\
\hline 12 & 60 & - & - & 2,02 & 5,77 \\
\hline 13 & 65 & - & - & 1,95 & 6,75 \\
\hline \multirow{2}{*}{\multicolumn{2}{|c|}{ arithm. }} & & & & \\
\hline & & 16,09 & 6,80 & 3,0 & 4,87 \\
\hline
\end{tabular}

SCHÄFFLER (1989) berichtet über zwei Stichprobenuntersuchungen von Fledermauskot des Großen Mausohres aus zwei verschiedenen Wochenstuben in der Region Ostalb (Baden-Württemberg). Angaben zum angewandten Untersuchungsverfahren liegen nicht vor. Auf der Grundlage der ermittelten Meßwerte leitet SCHÄFFLER eine 
hohe bis überhöhte Belastung des Fledermauskots durch Blei, Cadmium, Kupfer und Zink ab (Tab. 56). Beim Vergleich mit anderen Untersuchungen (ULOTH et al. 1987, RACKOW 1991, LACKI 1994) sowie den eigenen Analysen von 49 Kotproben können aber keine signifikanten Unterschiede festgestellt werden. Auch übersteigen die von SCHÄFFLER angegebenen Werte nicht die üblichen geogenen Backgroundgehalte der untersuchten Schwermetalle im Boden.

Tab. 56: Elementgehalte von zwei Kotstichproben aus Quartieren des Großen Mausohres (M. myotis) in der Region Ostalb (Baden-Württemberg). Nach SCHÄFFLER (1989)

\begin{tabular}{lccc}
\hline \hline \multicolumn{1}{c}{ Element } & Einheit & Kolonie 1 & Kolonie 2 \\
\hline Datum der & & & \\
Probenahme & & 28.09 .1985 & 15.05 .1988 \\
Blei & {$[\mathrm{mg} / \mathrm{kg}$ TS] } & 3,3 & 10 \\
Cadmium & {$[\mathrm{mg} / \mathrm{kg} \mathrm{TS}]$} & 3,1 & $<1$ \\
Chrom & {$[\mathrm{mg} / \mathrm{kg}$ TS] } & 0,6 & $<1$ \\
Eisen & {$[\mathrm{mg} / \mathrm{kg} \mathrm{TS}]$} & k.A. & 320 \\
Kupfer & {$[\mathrm{mg} / \mathrm{kg}$ TS] } & 43,6 & 51 \\
Mangan & {$[\mathrm{mg} / \mathrm{kg}$ TS] } & k.A. & 290 \\
Nickel & {$[\mathrm{mg} / \mathrm{kg}$ TS] } & 1,8 & $<1$ \\
Quecksilber & {$[\mathrm{mg} / \mathrm{kg}$ TS] } & 0,7 & 1,1 \\
Silber & {$[\mathrm{mg} / \mathrm{kg}$ TS] } & k.A. & $<1$ \\
Thallium & {$[\mathrm{mg} / \mathrm{kg}$ TS] } & k.A. & $<0,1$ \\
Zink & {$[\mathrm{mg} / \mathrm{kg}$ TS] } & 850 & 300 \\
\hline \hline k.A. $=$ keine Angabe & & &
\end{tabular}

PetiT und AlTENBACH (1973) untersuchten den Quecksilbergehalt im Kot der Brasilianischen Bulldoggfledermaus (Tardarida brasiliensis). Die Kotproben entstammten einer Höhle etwa 5,25 Meilen südwestlich einer großen Kupferhütte nahe Morenci, Arizona (USA). Der Probenaufschluß erfolgte durch Naßveraschung mit einem Salpetersäure-Schwefelsäuregemisch unter gelegentlicher Zugabe von Kaliumpermanganat. Die Analytik erfolgte mittels Kaltdampf-AAS. Die entnommenen Kotproben wurden stratigraphisch eingeordnet und die ermittelten Quecksilbergehalte mit der im gleichen Zeitraum produzierten Kupfermenge der Hütte verglichen. Hierbei ergab sich eine hohe Übereinstimmung zwischen dem Quecksilbergehalt im Kot und der produzierten Kupfermenge (Abb. 19). Die starken Produktionsrückgänge in den Jahren 1959 und 1967 sind die Folge von langanhaltenden Arbeitskämpfen gewesen. Während in Phasen geringer Produktion der Quecksilbergehalt im Kot um 0,8 mg/kg TS betrug, wurden in Phasen hoher Auslastung der Kupferhütte annähernd doppelt so hohe Quecksilbergehalte im Kot ermittelt. Die zeitliche Differenz zwischen dem Auftreten minimaler Quecksilbergehalte im Kot und geringer Kupferproduktion betrug lediglich ein Jahr. Dies belegt, wie rasch emittierte Schwermetalle auch in den Nahrungskreislauf von nicht bodenlebenden Säugetieren gelangen können.

CLARK et al. (1986) untersuchten im Rahmen von Biomonitoringstudien im Umfeld einer Batterieproduktionsanlage neben Gewebeproben von Myotis austroriparius 
auch stichprobenartig Kotproben. Der Kot war dabei sowohl von M. austroriparius wie auch von M. grisescens produziert worden. Alle Proben stammten aus dem Bereich Jackson Country (Florida, USA). Zum Vergleich wird noch über eine Kotprobe von Eptesicus fuscus berichtet, die ebenfalls aus der Region Jackson Country stammt, jedoch ein Jahr später (1982) entnommen wurde. Die referierten Daten für Cadmium, Chrom und Zink schwanken zwischen den verschiedenen Quartieren stark, aber gleichläufig. Die Bleikonzentration verhält sich hingegen genau gegenläufig zu den übrigen untersuchten Schwermetallkonzentrationen. Proben mit höheren Bleigehalten wiesen niedrigere Cadmium-, Chrom- und Zinkgehalte auf (Tab. 57).

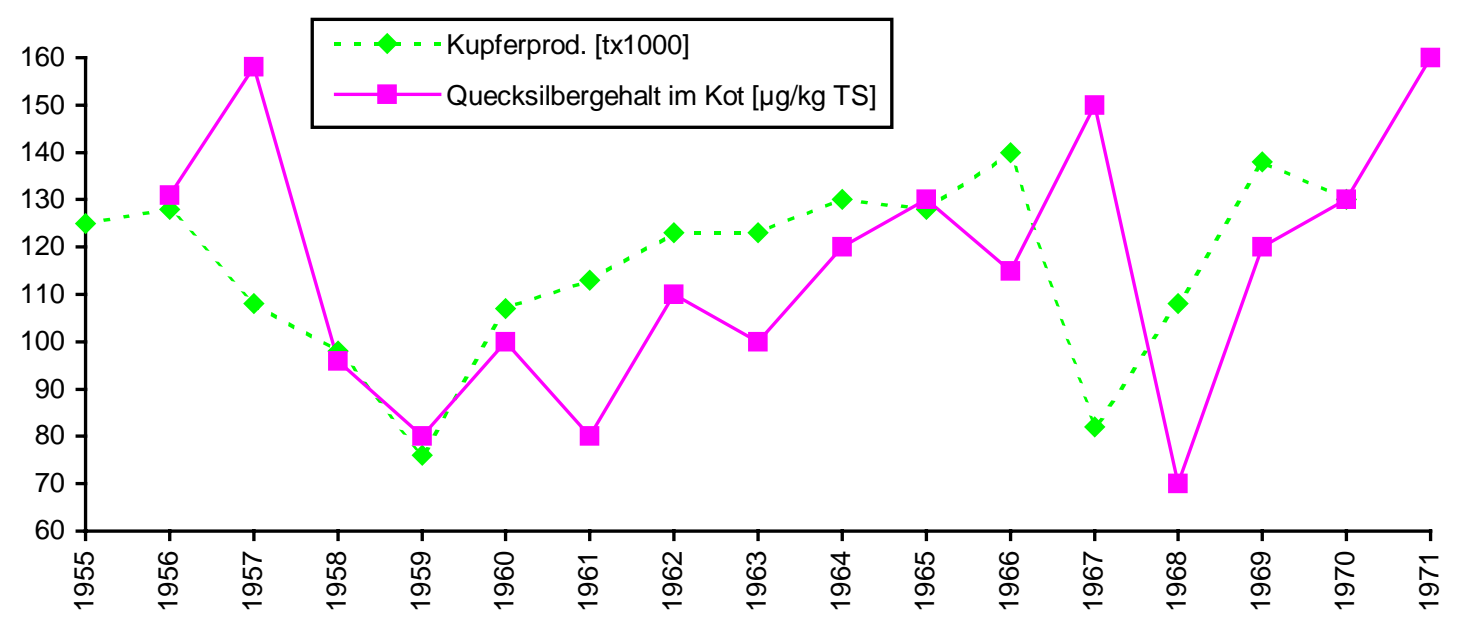

Abb. 19: Entwicklung der Quecksilbergehalte im Kot von Tadarida brasiliensis und der produzierten Kupfermenge einer Kupferhütte bei Morenci, Arizona (USA) nach den Untersuchungen von PETIT und ALTENBACH (1973).

Tab. 57: Schwermetallgehalte in Fledermauskot aus der Region Jackson Country (Florida, USA). Nach CLARK et al. (1986); alle Meßwerte in mg/kg TS

\begin{tabular}{|c|c|c|c|c|c|}
\hline Herkunft & Art & Blei & Cadmium & Chrom & Zink \\
\hline Judge Cave & $\begin{array}{l}\text { M. grisescens + } \\
\text { M. austroriparius }\end{array}$ & 3,4 & 2,2 & 2,7 & 640 \\
\hline Geromes C. & $\begin{array}{l}\text { M. grisescens + } \\
\text { M. austroriparius }\end{array}$ & 6,1 & 1,9 & 0,83 & 390 \\
\hline Sneads C. & $\begin{array}{l}\text { M. grisescens + } \\
\text { M. austroriparius }\end{array}$ & 3,9 & 2,3 & 5,0 & 530 \\
\hline PWRC* $^{*}$ & Eptesicus fuscus & 7,1 & 0,30 & 0,54 & 340 \\
\hline
\end{tabular}

*) Patuxent Wildlife Research Center 
LACKI (1994) untersuchte jeweils eine Kotstichprobe aus dem Jahre 1993 von zwei Clustern von Myotis grisescens aus einer Höhle bei Kentucky. Der Probenaufschluß erfolgte mit Salpetersäure, die Analysen mittels AAS. Für die Quecksilberbestimmung wurde ein Säuregemisch aus Kaliumpermanganat und -persulfat mit Schwefelund Salpetersäure verwendet (Tab. 58).

Tab. 58: Elementgehalte von zwei Kotstichproben aus einem Quartier von Myotis grisescens in Jessamine County, Kentucky (USA). Nach LACKY (1994)

\begin{tabular}{lccc}
\hline \hline \multicolumn{1}{c}{ Element } & Einheit & vorderes Cluster & hinteres Cluster \\
\hline Arsen & {$[\mathrm{mg} / \mathrm{kg} \mathrm{TS}]$} & 1,6 & 1,4 \\
Blei & {$[\mathrm{mg} / \mathrm{kg} \mathrm{TS}]$} & 3,6 & 2,7 \\
Cadmium & {$[\mathrm{mg} / \mathrm{kg} \mathrm{TS}]$} & $<5,5$ & $<6,5$ \\
Eisen & {$[\mathrm{mg} / \mathrm{kg}$ TS] } & 851,1 & 565,5 \\
Kupfer & {$[\mathrm{mg} / \mathrm{kg} \mathrm{TS}]$} & 73,6 & 69,1 \\
Mangan & {$[\mathrm{mg} / \mathrm{kg} \mathrm{TS}]$} & 66,3 & 84,7 \\
Nickel & {$[\mathrm{mg} / \mathrm{kg} \mathrm{TS}]$} & $<14$ & $<16$ \\
Quecksilber & {$[\mathrm{mg} / \mathrm{kg}$ TS] } & 0,15 & $<0,1$ \\
Selen & {$[\mathrm{mg} / \mathrm{kg}$ TS] } & 13,7 & 15,6 \\
Silber & {$[\mathrm{mg} / \mathrm{kg}$ TS] } & $<5,5$ & $<6,5$ \\
Zink & {$[\mathrm{mg} / \mathrm{kg}$ TS] } & 320,1 & 355,5 \\
\hline \hline
\end{tabular}

\subsection{Ableitung von Referenzbereichen}

Auf der Grundlage der eigenen Untersuchungsergebnisse erfolgt die Herleitung von Referenzbereichen. Die Referenzbereiche kennzeichnen den Konzentrationsbereich, der bei nicht anthropogen bzw. geogen bedingter erhöhter Schwermetallaufnahme in dem jeweiligen Organ bzw. in Knochen, Haaren oder Kot zu erwarten ist. Als Referenzbereich wurde der Konzentrationsbereich zwischen dem oberen und unteren Quartil gewählt, d.h. der Bereich, in dem sich bei den vorliegenden Untersuchungen genau $50 \%$ aller untersuchten Tiere befanden. Bei der Ableitung der Referenzwerte wurden Tiere mit bekannter Vorbelastung, wie für Blei die Tiere aus dem Bereich des Harzes, nicht mit berücksichtigt. Zur Vermeidung von Scheingenauigkeiten werden die Referenzbereiche überwiegend nur mit einer signifikanten Stelle angegeben. Die Auswahl der Klassenbreite zur Festlegung eines Referenzbereiches ist problematisch und kann in gewissem Umfange immer als willkürlich interpretiert werden. Aufgrund der ausschließlich rechtsschiefen Verteilungen der Schwermetallgehalte des untersuchten Probenkollektives und der vergleichsweise noch geringen Stichprobengröße würde die Ausweitung der Klassenbreite, z.B. vom 10. bis zum 90. Perzentil, überproportional viele Individuen mit erhöhten Schwermetallgehalten erfassen. Aus diesem Grunde wurde die Festlegung der Referenzbereiche auf der Grundlage der Quartilswerte getroffen.

Die Übertragbarkeit der von den Untersuchungen an Zwergfledermäusen abgeleiteten Referenzbereiche auf andere Fledermausarten ist nicht ganz unproblematisch, 
wie der Unterschied der Knochenbleigehalte bei Zwergfledermäusen im Vergleich zu Großen Mausohren zeigte (Kap. 3.6). Zumindest zur Beschreibung eines groben Richtwertes erscheint eine Übertragbarkeit auf andere Arten aber möglich, wie die nachstehenden Vergleiche mit den referierten Literaturdaten (Kap. 4.2.2) zeigen. Bei dieser Gegenüberstellung der Literaturdaten wurden nur solche Werte berücksichtigt, bei denen nicht explizit ein Zusammenhang zu Schwermetallintoxikationen beschrieben ist.

\subsubsection{Knochen}

Die Berechnung der Referenzbereiche für den Bleigehalt in Knochen erfolgte ausschließlich auf der Basis der harzfernen Fledermäuse der Jahre 1996-1999. Aufgrund der nachgewiesenen unterschiedlichen Knochenbleigehalte zwischen kleinen Arten wie der Zwergfledermaus und großen Arten wie dem Großen Mausohr bezieht sich der errechnete Referenzbereich bei enger Auslegung nur auf die Zwergfledermäuse. Aufgrund der bekannten Schwankungen des Bleigehaltes in Abhängigkeit des Knochentyps (BARRY 1975) sollten zudem nur Unterarmknochen miteinander verglichen werden.

Der Referenzbereich für den Knochenbleigehalt beträgt unter Berücksichtigung der zuvor gemachten Einschränkungen 7-17 $\mu \mathrm{g} / \mathrm{g}$ TS.

\subsubsection{Organe}

\section{Blei:}

Zur Ableitung von Referenzbereichen wurden ausschließlich die untersuchten Tiere aus harzfernen Bereichen herangezogen. Für die einzelnen Organe wurden folgende Referenzbereiche errechnet:

Lungengewebe: $\quad 0,5-0,9 \mu \mathrm{\mu} / \mathrm{g}$ TS

Lebergewebe: $\quad 1,2-4,7 \quad \mu \mathrm{g} / \mathrm{g}$ TS

Nierengewebe: $\quad 0,6-1,5 \mu \mathrm{\mu} / \mathrm{g}$ TS

Im Vergleich zu den verfügbaren Literaturwerten spiegeln die errechneten Referenzbereiche jeweils den unteren Spannweitenbereich der verschiedenen Untersuchungen wider. Für das Lebergewebe zeigt sich, daß die mittleren Bleigehalte bei den Untersuchungen von StREIT und NAGEL (1993a, b) und THIES und GREGORY (1994) in dem errechneten Referenzbereich liegen (Abb. 20). 

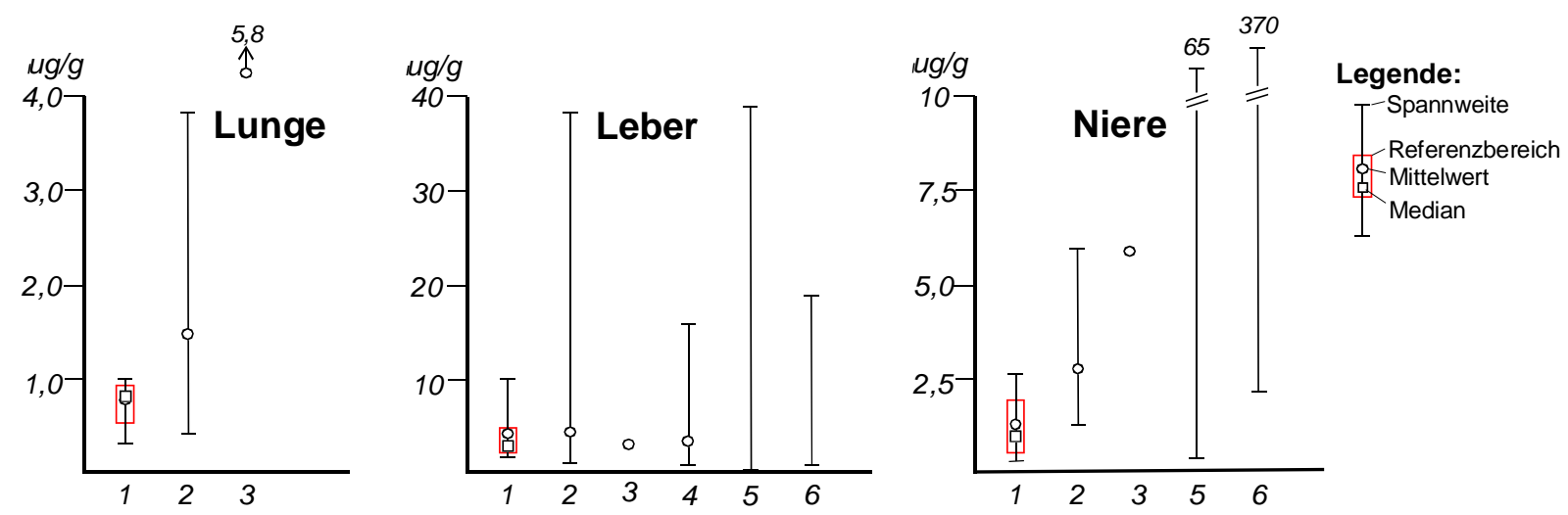

Abb. 20: Vergleich der errechneten Referenzwerte für Blei in Gewebeproben mit publizierten Literaturdaten.

1= diese Arbeit, Meßwerte auf TS bezogen; 2=STREIT und NAGEL (1993a), Meßwerte auf TS bezogen; 3=STREIT und NAGEL (1993b), Meßwerte auf TS bezogen; 4=THIES und GREGORY (1994), tropische Art, Meßwert auf FS bezogen; $5=$ SUTTON und HARIONO (1986), tropische Art, Meßwert auf FS bezogen; 6=SKERRATT et al. (1998), keine Angabe der Bezugsgröße.

\section{Cadmium:}

Aus den eigenen Untersuchungen lassen sich unter Berücksichtigung aller untersuchten Tiere folgende Referenzbereiche ableiten:

Lebergewebe: $\quad 0,05-1,4 \quad \mu \mathrm{g} / \mathrm{g}$ TS

Nierengewebe: $\quad 0,2-2,7 \quad \mu \mathrm{g} / \mathrm{g}$ TS

Die errechneten Referenzbereiche umschließen alle publizierten Mittelwerte für Cadmium und liegen wie beim Blei im unteren Spannweitenbereich der Vergleichsdaten, dies ist typisch für rechtsschiefe Verteilungen (Abb. 21).

\section{Chrom:}

Aus den eigenen Untersuchungen lassen sich unter Berücksichtigung aller untersuchten Tiere folgende Referenzbereiche für Chrom, ges. ableiten:

Lebergewebe: $\quad 0,02-0,05 \mu \mathrm{\mu g} / \mathrm{g}$ TS

Nierengewebe: $\quad 0,02-0,2 \quad \mu \mathrm{g} / \mathrm{g}$ TS

Vergleichsdaten zu Chromgehalten in Gewebeproben von Fledermäusen finden sich in der Literatur kaum. Lediglich in den Untersuchungen von STREIT und NAGEL (1993a und 1993b) finden sich Angaben zu Chromgehalten u.a. in Leber- und Nierengewebe. Diese Werte liegen etwa 10-fach über den eigenen Daten. 

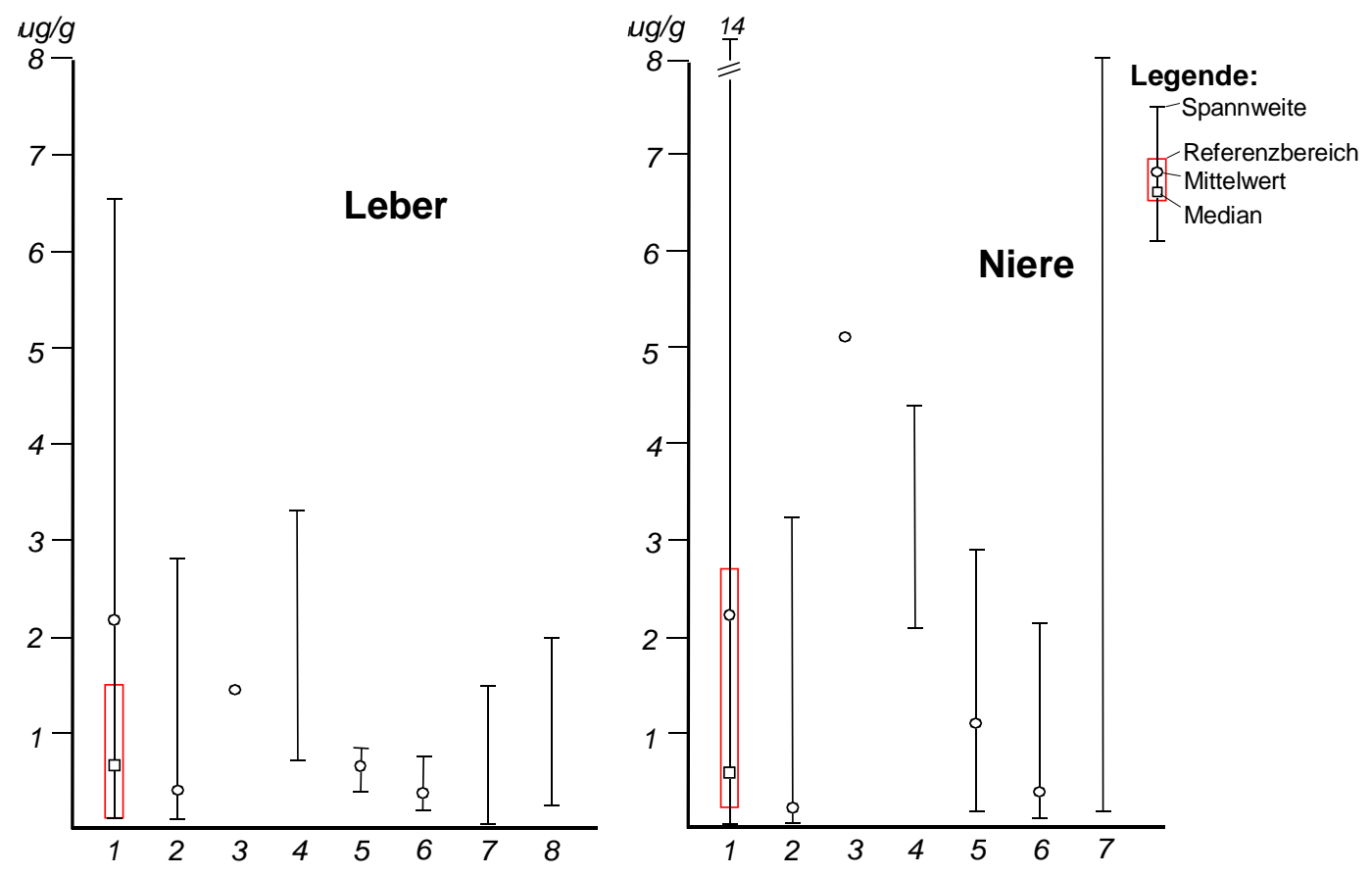

Abb. 21: Vergleich der errechneten Referenzwerte für Cadmium in Gewebeproben mit publizierten Literaturdaten.

1= Diese Arbeit, Meßwerte auf TS bezogen; 2=STREIT und NAGEL (1993a), Meßwerte auf TS bezogen; 3=STREIT und NAGEL (1993b), Meßwerte auf TS bezogen; 4=GERELL und GERELL (1993), Zwergfledermäuse, Meßwert auf TS bezogen; $5=$ CLARK et al. (1986), Judges Cave, Meßwert auf FS bezogen; 6=CLARK et al. (1986), Gainesville, Meßwert auf FS bezogen, 7=SKERRATT et al. (1998), tropische Art, Bezugsgröße unbekannt; 8=THIES und GREGORY (1994), tropische Art, Meßwert auf FS bezogen.

\subsubsection{Haare}

Zur Berechnung der Referenzbereiche der Schwermetallgehalte im Haar der Zwergfledermäuse wurde für die Elemente Blei und Nickel aufgrund der erkennbaren Konzentrationsabnahme während des Betrachtungszeitraumes die Datensätze der Jahre 1996 bis 1999 verwendet. Die Berechnung der Referenzwerte für die übrigen Elemente erfolgte unter Einbeziehung aller Meßwerte mit Ausnahme der Datensätze mit Zinkgehalten oberhalb von $1000 \mathrm{mg} / \mathrm{kg}$ TS.

Für die untersuchten Elemente errechnen sich folgende Referenzbereich im Haar (jeweils bezogen auf die bei $50 \%$ rel. Luftfeuchtigkeit äquilibrierten Haare):

Blei: $\quad 8-52 \quad \mu \mathrm{g} / \mathrm{g}$

Cadmium: $\quad 0,15-0,55 \mu \mathrm{g} / \mathrm{g}$

Chrom, ges.: $1,2-3,5 \mu \mathrm{g} / \mathrm{g}$

Kupfer: $\quad 13-36 \quad \mu \mathrm{g} / \mathrm{g}$

Nickel: $\quad 1,2-2,6 \quad \mu \mathrm{g} / \mathrm{g}$

Zink: $\quad 230-390 \mu \mathrm{m} / \mathrm{g}$ 
Beim Vergleich der errechneten Referenzbereiche mit den Literaturangaben anderer Autoren läßt sich zu den Untersuchungen von STREIT und NAGEL (1993a) an zehn Fledermäusen aus sechs Taxa eine gute Übereinstimmung feststellen (vgl. Kap. 4.2.2, Tab. 41). Die an Haarproben des Großen Mausohres von ZYRNICKI et al. (1996) ermittelten Kupfer und Zinkgehalte weichen hingegen wesentlich von den vorgenannten Referenzbereichen ab. Diese Daten fallen jedoch bereits durch stark abweichende Aluminium- und Zinkgehalte von anderen an Säugetieren erhobenen Haarelementwerten auf (vgl. Kap. 4.2.2, Tab. 44).

Im Vergleich zum Humanhaar ergibt sich mit Ausnahme des Bleis für alle anderen untersuchten Schwermetalle eine gute Übereinstimmung (KIJEWSKI 1993). Der Bleigehalt im Fledermaushaar liegt hingegen deutlich höher. KIJEWSKI (1993) gibt für das 5. und 95. Perzentil Bleigehalte im Humanhaar von $0,2 \mu \mathrm{g} / \mathrm{g}$ bis $8 \mu \mathrm{g} / \mathrm{g}$ an.

\subsubsection{Kot}

Die Berechnung der Referenzbereiche für Fledermauskot erfolgte unter Ausschluß der nicht aus Niedersachsen stammenden Daten (Probennr. 38 und 39) sowie unter Ausschluß der aus dem Harz stammenden Daten (Probennr. 21, 22, 24, 56). Die errechneten Referenzbereiche betragen wie folgt:

$\begin{array}{lll}\text { Arsen, ges.: } & 2,1-5,2 & \mathrm{mg} / \mathrm{kg} \text { TS } \\ \text { Blei: } & 3,2-7,7 & \mathrm{mg} / \mathrm{kg} \text { TS } \\ \text { Cadmium: } & 1,6-3,2 & \mathrm{mg} / \mathrm{kg} \text { TS } \\ \text { Chrom, ges.: } & 1,6-3,6 & \mathrm{mg} / \mathrm{kg} \text { TS } \\ \text { Kupfer: } & 77-120 & \mathrm{mg} / \mathrm{kg} \text { TS } \\ \text { Nickel: } & 1,5-3,5 & \mathrm{mg} / \mathrm{kg} \text { TS } \\ \text { Zink: } & 460-900 & \mathrm{mg} / \mathrm{kg} \text { TS }\end{array}$

\subsection{Auswirkungen der Substitution verbleiter Kraftstoffe auf die Bleibelastung der Fledermäuse}

Das in unterschiedlichen Umweltkompartimenten nachweisbare Blei entstammt zu großen Teilen den Kraftfahrzeugemissionen. Ab 1923 wurden dem Vergaserkraftstoff Bleiverbindungen zugesetzt, um dessen Klopffestigkeit zu erhöhen. Hierbei handelte es sich um Blei in Form von organisch gebundenen Bleiverbindungen, vor allem Tetraethylblei und untergeordnet Tetramethylblei. Rund $75 \%$ des im Vergaserkraftstoff enthaltenen Bleis gelangten mit den Abgasen überwiegend partikelgebunden oder in Form von Aerosolen in die Atmosphäre, woraus eine nahezu ubiquitäre Verbreitung des Bleis in der Umwelt resultierte. Im Jahre 1959 wurden bereits erste Warnungen hinsichtlich negativer Gesundheitseffekte in Folge der global steigenden Bleigehalte in allen Umweltkompartimenten geäußert. Dennoch dauerte es weitere 25 Jahre, bis in den führenden Industrienationen bleifreies Benzin überall 
und in ausreichender Menge verfügbar war. Vorangegangen waren unzählige Studien und Hearings über die potentiellen Auswirkungen steigender Bleigehalte in der Umwelt, aber auch über die essentielle Bedeutung von Blei, mit der noch in den siebziger Jahren von interessierter Seite ein Verbot von bleihaltigem Vergaserkraftstoff verhindert werden sollte, wie NEEDLEMAN (2000) berichtet.

In der Bundesrepublik Deutschland wurde mit der Einführung der ersten Stufe des Benzinbleigesetzes (BzBIG vom 5.8.1971) ab 1.1.1972 der Bleigehalt im Vergaserkraftstoff auf $400 \mathrm{mg} / \mathrm{l}$ begrenzt, mit Inkrafttreten der zweiten Stufe des Benzinbleigesetzes erfolgte ab 1.1.1976 eine weitere Reduzierung des Bleigehaltes im Vergaserkraftstoff auf $150 \mathrm{mg} / \mathrm{l}$. Seit dem 1.2.1988 ist der Vertrieb von bleihaltigem Normalbenzin $(\mathrm{ROZ}<95)$ verboten. Bis zu diesem Zeitpunkt betrug der Anteil an verbleitem Vergaserkraftstoff noch rund $74 \%$. Bleifreies Benzin darf heute maximal $13 \mathrm{mg} / \mathrm{l}$ Blei enthalten (Benzinbleigesetz i.d.F. vom 24.6.1994). Im Jahre 1997 betrug der Absatz verbleiten Vergaserkraftstoffes nur noch 0,025\% des gesamten Vergaserkraftstoffverbrauches von rund 30 Mio t/a in der Bundesrepublik Deutschland (UMWELTBUNDESAMT 2000).

Im Jahre 1987 wurden nach den Erhebungen des Umweltbundesamtes (UMWELTBUNDESAMT 1991) noch 2900 t Blei über Fahrzeugabgase (ohne Hochseeschiffe, Kraftwerke und Eisenbahnen) in den alten Bundesländern emittiert. Dieser Bleiausstoß reduzierte sich mit dem Verbot von bleihaltigem Normalbenzin ab dem 1.2.1988 rasch auf 1700 t/a für das Jahr 1989. Für das Jahr 1997 kann die über Kraftfahrzeugemissionen freigesetzte Bleimenge auf unter $500 \mathrm{t} / \mathrm{a}$ abgeschätzt werden.

In der Folge der sinkenden Bleiemissionen nahm auch die Bleibelastung der Biosphäre nachweislich ab. Dies führte auch zum Rückgang der inkorporierten Bleimenge bei Tieren und beim Menschen.

WIETLISBACH et al. (1995) untersuchten den Blutbleigehalt der Schweizer Bevölkerung während der Übergangsperiode von verbleitem Vergaserkraftstoff zu unverbleitem Kraftstoff und ermittelten dabei einen Rückgang der Blutbleiwerte um rund $50 \%$ (Tab. 59). Zu ähnlichen Ergebnissen kommen Studien im Auftrage des Umweltbundesamtes (UmweltBUndesamt 1997). Die Blutbleibelastung (Vollblut) von Studentenkollektiven (Männer und Frauen) der Universität Münster nahm von etwa $80 \mu \mathrm{g} / \mathrm{l}$ im Jahre 1984/85 auf rund 31 $\mu \mathrm{g} / \mathrm{lim}$ Jahr $1995 \mathrm{ab}$. Die Abnahme der Bleikonzentrationen im Haar zeigte sich bei demselben Kollektiv hingegen verzögert und weniger ausgeprägt (vgl. Tab. 59). Für Nordamerika belegen Studien der USEPA ebenfalls einen Rückgang der Blutbleibelastung im Menschen von rund $50 \%$ infolge der Substitution des verbleiten Vergaserkrafstoffes (USEPA 1986 zit. n. NEEDLEMAN 2000). 
Tab. 59: Entwicklung der Bleigehalte (Medianwerte) im Vollblut (Männer) und Haar beim Menschen während der Übergangsperiode von verbleitem Vergaserkraftstoff zu unverbleitem Kraftstoff nach WIETLISBACH et al. (1995) und UMWELTBUNDESAMT (1997)

\begin{tabular}{cccc}
\hline \hline Betrachtungszeitraum & $\begin{array}{c}\text { Blutbleigehalte } \\
{[\mu \mathrm{g} / \mathrm{l}]} \\
\text { Schweiz }\end{array}$ & $\begin{array}{c}\text { Blutbleigehalte } \\
{[\mu \mathrm{\mu} / \mathrm{l}]} \\
\text { Deutschland }\end{array}$ & $\begin{array}{c}\text { Haarbleigehalte }[\mu \mathrm{g} / \mathrm{g}] \\
\text { Deutschland }\end{array}$ \\
\hline $1984-1985$ & 120 & 87 & 1,15 \\
$1988-1989$ & 87 & 59 & 0,68 \\
$1992-1993$ & 68 & 50 & 0,50 \\
\hline \hline
\end{tabular}

Der Rückgang der Knochenbleigehalte in den untersuchten Zwergfledermäusen liegt in ähnlicher Größenordnung. Die Knochenbleibelastung nahm hier von $18 \mu \mathrm{g} / \mathrm{g}$ TS in der 2. Hälfte der achtziger Jahre auf $9 \mathrm{mg} / \mathrm{kg}$ in der 2. Hälfte der neunziger Jahre ab, dies entspricht einem Rückgang um $50 \%$. Ein ähnlicher Trend kann bei der Haarbleibelastung festgestellt werden. Hier ergab sich im Zeitraum 1987 bis 1999 ein Rückgang der Medianwerte der Haarbleikonzentrationen von $56 \mu \mathrm{g} / \mathrm{g}$ auf $23 \mu \mathrm{g} / \mathrm{g}$, dies entspricht einem prozentualem Rückgang von rund $55 \%$.

Die für zahlreiche Umweltkompartimente bis hin zu Humanproben erkennbare Reduzierung der Bleibelastung nach Umsetzung der letzten Stufe des Benzinbleigesetzes am 1.2.1988 führte somit auch bei den heimischen Fledermäusen zu einer signifikanten Reduzierung der Bleibelastung.

\subsection{Auswirkungen von Bodenbelastungen auf die Schwermetallbelastung der Fledermäuse}

Die festgestellten erhöhten Bleigehalte in Fledermäusen aus der Region des Harzes steht in Übereinstimmung mit den bekannten hohen Bodenbelastungen in der Harzregion insbesondere durch Blei und Zink. Besonders der Westharz stellt eine historische Industrieregion mit einer fast 1000-jährigen Bergbautätigkeit dar (BARTELS 1992, SEGERS-GLOCKE 2000). Für den Bereich des Rammelsberges bei Goslar belegen Funde aus der Zeit um 1000 v.Chr. (Funde von Müllingen) bereits die Gewinnung von Kupfer (BACHMANN et al. 2000). Ausgebeutet wurden vor allem die oft silberreichen Blei- und Zinkerzlagerstätten. Das anfallende Erz wurde nach der Aufbereitung sogleich vor Ort verhüttet. BODE (1928) erfaßte 189 ehemalige Hüttenplätze, die seit dem frühen Mittelalter angelegt wurden und unterschiedlich lange produzierten. Die Hüttenbetriebe im Harz waren bis in die siebziger Jahre des 20. Jahrhundert in Betrieb (LEHNE und WEINBERG 1968), die Bergbaubetriebe sogar bis zu Beginn der neunziger Jahre des 20. Jahrhunderts. Gegenwärtig findet die Verhüttung von Blei und Zink nur noch im Bereich von Oker am Nordrand des Harzes bei Goslar statt. In 
der Folge der ehemaligen intensiven Industrialisierung der Region des Harzes gelangten mit der Erzaufbereitung und der Verhüttung große Mengen an Schwermetallen, vor allem an leichtflüchtigen Elementen wie Quecksilber, Arsen oder Cadmium, aber auch Schwefel- und Stickoxide in die Biosphäre. Die Folge waren weitflächig entwaldete Regionen, so z.B. im Innerstetal aufgrund der z.T. extremen Deposition saurer Niederschläge und schwermetallhaltiger Stäube und Aerosole. SCHROEDER und REUSS (1883) beschreiben anschaulich die damaligen Auswirkungen.

In neuerer Zeit hat besonders die Verfrachtung von schwermetallhaltigen Sedimenten in das Harzvorland aus Bodenschutzgründen verstärkte Beachtung gefunden (Lindorfer 1997, BuRKARD 1999, DeICKE und RUPPERT 2000, NiedERSÄCHSISCHES LANDESAMT FÜR BODENFORSCHUNG 2000).

Die früheren Emissionen schwermetallhaltiger Stäube und Aerosole, aber auch von schwermetallhaltigen Wässern aus der Erzaufbereitung, führten bis heute in den Flußniederungen der Harztäler, aber auch in den Überflutungsbereichen im Harzvorland bis nach Hannover zu erhöhten Schwermetallgehalten im Boden und in den Sedimenten (NowaK und PreUl 1971, HoRN 1974, FytianOS 1978, StefFen 1994, ArGE REINHALTUNG Weser 1996). Während in unbelasteten Regionen Niedersachsens der Bleigehalt in Auensedimenten etwa um $80 \mathrm{mg} / \mathrm{kg}$ TS beträgt (SCHNEIDER 1999), liegen die Bleigehalte in Böden des Harzes oberhalb von $1000 \mathrm{mg} / \mathrm{kg}$ TS (BÜTTNER 1997, BARTENS und BÜTTNER 1997). Nicht selten werden Gehalte bis über $4.000 \mathrm{mg} / \mathrm{kg}$ TS ermittelt (HARRE und WALTHER 1957, ZÜLL 1995, eigene Untersuchungen).

Die hohen Schwermetallgehalte im Boden führten zu Verzehrs- und Anbauempfehlungen für Grundeigentümer und Kleingartenbesitzer im Bereich des Harzes. Die Bodenbelastungen spiegeln sich aber auch in den Elementgehalten von Säugetieren wider, wie diese Untersuchung am Beispiel der Fledermäuse zeigte. KNOLLE und KNOLLE (1983) beschrieben für den Bereich des Harzes Verluste bei Brutvögel und Wasservögel infolge Schwermetallvergiftung.

Erhöhte Schwermetallgehalte in Säugetieren aus dem Harz wurden bereits früh für Haustiere und jagdbare Wildarten beschrieben. Während erste Erwähnungen akute Vergiftungsfälle beschreiben (GATTERER 1786, MEYER 1822) beschreiben spätere Untersuchungen auch chronische Erkrankungen bei Nutztieren infolge hoher Schwermetallinkorporation, wie z.B. die Schleuderkrankheit beim Rotwild (REICHSBUNd DeUtSCHE JÄGERSCHAFT 1939). KLEIMINGER (1983) untersuchte an Rehen (Capreolus capreolus L.), Rotwild (Cervus elaphus L.), Schwarzwild (Sus scrofa L.) sowie Feldhasen (Lepus europaeus P.) aus fünf Untersuchungsräumen in niedersächsischen Jagdrevieren die Gehalte an Arsen, Blei, Cadmium und Quecksilber. Einer der Untersuchungsräume umfaßte das Gebiet des Westharzes, ein weiterer Untersuchungsraum den Bereich Göttingen-Northeim. Beim Vergleich der Meßwerte für Blei ergibt sich in nahezu allen Fällen ein signifikant höherer Bleigehalt in den Wildproben aus dem Bereich des Harzes (Tab. 60). 
Tab. 60: Medianwerte und Spannweiten (in Klammern) der Bleigehalte in Gewebeproben von jagdbaren Wildarten aus dem Bereich des niedersächsischen Harzes (Westharz) und Vergleichsregionen nördlich des Harzes zwischen Braunschweig, Peine und Salzgitter sowie Lüchow-Dannenberg und südlich des Harzes zwischen Göttingen und Northeim. Nach KLEIMINGER (1983). Alle Meßwerte in $\mathrm{mg} / \mathrm{kg}$ FS

a) Rehwild:

\begin{tabular}{lccc}
\hline \multicolumn{1}{c}{ Region } & Haare & Leber & Nieren \\
\hline Harz & 0,55 & 0,23 & 0,15 \\
südl. Niedersachsen & $(<\mathrm{BG}-13)$ & $(0,012-4,6)$ & $(0,053-1,3)$ \\
& 0,40 & 0,13 & 0,15 \\
Braunschweig-Peine- & $(<\mathrm{BG}-48)$ & $(0,018-14)$ & $(0,028-1,8)$ \\
Salzgitter & 0,42 & 0,14 & 0,19 \\
Lüchow-Dannenberg & $(<\mathrm{BG}-16)$ & $(0,026-2,6)$ & $(0,021-1,7)$ \\
& 0,81 & 0,11 & 0,13 \\
& $(0,080-70)$ & $(0,017-1,9)$ & $(0,014-0,44)$ \\
\hline \hline
\end{tabular}

b) Rotwild:

\begin{tabular}{lccc}
\hline \hline \multicolumn{1}{c}{ Region } & Haare & Leber & Nieren \\
\hline Harz & 1,7 & 0,79 & 0,27 \\
Lüchow-Dannenberg & $(0,11-55)$ & $(<\mathrm{BG}-6,7)$ & $(0,17-0,59)$ \\
& 0,89 & 0,18 & 0,19 \\
& $(0,30-3,0)$ & $(0,050-0,57)$ & $(0,021-1,7)$ \\
\hline \hline
\end{tabular}

c) Schwarzwild:

\begin{tabular}{lccc}
\hline \hline \multicolumn{1}{c}{ Region } & Haare & Leber & Nieren \\
\hline Harz & 9,1 & 0,11 & 1,7 \\
& $(2,4-19)$ & $(0,42-4,9)$ & $(0,42-3,6)$ \\
südl. Niedersachsen & 2,1 & 0,24 & 0,36 \\
& $(1,5-11)$ & $(0,13-0,60)$ & $(0,16-0,87)$ \\
Braunschweig-Peine- & 1,6 & 0,13 & 0,15 \\
Salzgitter & $(0,25-30)$ & $(0,039-1,1)$ & $(0,004-1,3)$ \\
Lüchow-Dannenberg & 1,6 & 0,29 & 0,31 \\
& $(0,21-18)$ & $(0,090-1,3)$ & $(0,070-0,62)$ \\
\hline \hline
\end{tabular}

d) Feldhase:

\begin{tabular}{lccc}
\hline \hline \multicolumn{1}{c}{ Region } & Haare & Leber & Nieren \\
\hline Harz & 3,3 & 5,8 & 4,4 \\
& $(1,8-11)$ & $(1,0-13)$ & $(0,51-33)$ \\
südl. Niedersachsen & 0,48 & 1,1 & 0,51 \\
& $(0,10-2,0)$ & $(0,42-2,2)$ & $(0,27-1,6)$ \\
Braunschweig-Peine- & 1,3 & 1,1 & 0,69 \\
Salzgitter & $(<B G-100)$ & $(0,010-35)$ & $(0,059-26)$ \\
Lüchow-Dannenberg & 0,58 & 0,71 & 0,47 \\
& $(0,013-24)$ & $(0,10-2,1)$ & $(0,056-1,2)$ \\
\hline \hline
\end{tabular}


Die Werte von KLEIMINGER zeigen deutlich: Je enger ein Tier mit dem Boden in Kontakt steht, um so größer ist die Gewebebelastung durch Blei in Gebieten mit hohen Bodenbleigehalten. Vergleichbare Ergebnisse lieferten die Untersuchungen von LUTZ (1985) und LUTZ und SLAMECKA (1997) an Feldhasen und Rehen.

Im Gegensatz zu Fledermäusen nehmen die von KLEIMINGER untersuchten Säugetiere allesamt ihre Nahrung unmittelbar von der Geländeoberfläche auf. Die Wahrscheinlichkeit, daß hierbei Bodenpartikel oral mit aufgenommen werden, ist daher ungleich größer. Fledermäuse als Gipfelräuber in der Nahrungspyramide nehmen ihre Nahrung fast ausschließlich aus dem Luftraum auf (vgl. Kap. 1.3), wodurch eine direkte orale Aufnahme von Boden als vernachlässigbar zu beurteilen ist. Insofern muß die Inkorporation von Schwermetallen, speziell von Blei, über die Nahrung erfolgen. Die Schwermetallinkorporation über den Wasserpfad kann hingegen unberücksichtigt bleiben. Aufgrund der Bedeutung des Westharzes für die Trinkwassergewinnung laufen langjährige Schwermetallüberwachungsmaßnahmen an den Wässern der Talsperren und deren Zuläufe (ALIKE 1974). Diese Untersuchungen ergaben, auch unter Berücksichtigung der steigenden Bodenversauerung, bislang keine Hinweise auf erhöhte Schwermetallfrachten in gelöster Form oberhalb der Grenzwerte gemäß Trinkwasserverordnung. FYTIANOS (1978) errechnete für die von inm untersuchten Fließgewässer im Harz eine maximal 7-fache Anreicherung für Blei im Vergleich zu unbelasteten Binnengewässern (Tab. 61).

Tab. 61: Mittlere Schwermetallgehalte in Fließgewässern des Harzes nach FYTIANOS (1978). Alle Meßwerte in $\mu \mathrm{g} / \mathrm{l}$

\begin{tabular}{lrccc}
\hline \hline \multicolumn{1}{c}{ Gewässer } & Blei & Kupfer & Nickel & Zink \\
\hline unkontaminierte Referenz- & & & & \\
gewässer & 3 & 7 & 3 & 10 \\
Innerste & 15 & 4,1 & 5 & 570 \\
Sieber & 3 & 5 & 2 & 91 \\
Söse & 7 & 10 & 8 & 47 \\
\hline \hline
\end{tabular}

Zusätzlich zur Schadstoffaufnahme mit der Nahrung werden in schwermetallbelasteten Regionen bei der Fellpflege Schwermetalle aus den am Fell anhaftenden Stäuben aufgenommen. Die über den Luftpfad verursachte Schwermetallaufnahme kann hingegen als vernachlässigbar angenommen werden, wie die Untersuchung von Lungengewebe bei Zwergfledermäusen aus dem Bereich des Harzes sowie aus harzfernen Bereichen ergab.

Die vorliegenden Untersuchungen zeigen, daß Fledermäuse in Regionen mit erhöhten Schwermetallgehalten im Boden gleichfalls eine erhöhte Schwermetallbelastung im Organismus aufweisen. Speziell am Knochenbleigehalt konnte für Tiere aus dem Bereich des Harzes ein signifikant erhöhter Bleigehalt im Vergleich zu Tieren aus dem übrigen Niedersachsen ermittelt werden. Damit eignen sich auch Fledermäuse 
sehr wohl zur Indikation umweltrelevanter Schwermetallbelastungen. Die anderslautenden Aussagen von LEITNER und VOGEL (1993) können aufgrund methodischer Unzulänglichkeiten (Ganztieraufschlüsse, fehlende Berücksichtigung des Mumifizierungsgrades) als widerlegt beurteilt werden. Bei der Untersuchung der Schadstoffbelastung von Fledermäusen darf jedoch ausschließlich ein Vergleich zwischen einzelnen Geweben angestellt werden. Ganztieraufschlüsse, wie sie in der Literatur gelegentlich publiziert wurden, sind zur Beurteilung der Schadstoffbelastung ungeeignet.

\subsection{Auswirkungen wasserlöslicher Holzschutzmittel auf die Schwermetallbelastung von Fledermäusen}

Eine Belastung der Fledermäuse durch Schwermetalle muß nicht ausschließlich alimentär erfolgen. Auch ein direkter Kontakt mit schwermetallhaltigen Oberflächen kann zur Anreicherung im Organismus führen. Als wesentliche Quellen für diesen Expositionspfad kommen Holzschutzmittel auf Schwermetallsalzbasis in Betracht.

Holzschutzmittel auf Basis der im Rahmen dieser Untersuchungen analysierten Schwermetalle sind in der Bundesrepublik Deutschland überwiegend in Form von Gemischen anorganischer Salze von Chrom und Kupfer zugelassen (Tab. 62). Sie besitzen fungizide und insektizide Eigenschaften. Die Produktionsmenge für Deutschland beträgt nach LEIBE (1992) um 10.000 t/a. Aufgrund der Zulassung einer Großzahl dieser Produkte für den Anwendungsbereich GK 2 nach DIN 68 800-T3 04:1990 finden diese Produkte in großem Umfange auch Verwendung für die Behandlung von Dachgebälk und Fassadenlattungen, d.h. den wichtigsten Lebensräumen der sogenannten Hausfledermäuse wie der Zwergfledermaus.

Die vorgenannten Holzschutzmittel sind allesamt gut wasserlöslich. Dadurch ist auch ein gutes Eindringverhalten in frische Hölzer gegeben. Die enthaltenen Metallsalze reagieren unter Bildung schwerlöslicher komplexer Verbindungen mit dem Lignin und den Hemicellulosen des Holzes. Die Chromate werden zudem nach ihrer Aufbringung allmählich zu toxikologisch unbedenklicherem $\mathrm{Cr}^{3+}$ aufoxidiert, wodurch eine asymptotische Abnahme der Wasserlöslichkeit und damit der Bioverfügbarkeit eintritt. Die Auswaschrate von Chrom und Kupfer beträgt nach der Imprägnierung in einem Zeitraum von 4 Wochen bis zu 6-10 \% der Anwendungsmenge (LEIBE 1992, BRINGEZU 1992).

Aufgrund der hohen Toxizität und des cancerogenen Potentiales von Chrom-VIVerbindungen gegenüber Säugetieren stellen besonders frisch verarbeitete chromsalzimprägnierte Hölzer ein besonderes Risiko für die Gruppe der sogenannten Hausfledermäuse dar. Nach KALBERLAH (1999) beträgt die LD ${ }_{50}$-Dosis von Chrom-VIVerbindungen im Rattenversuch 80-177 mg/kg Körpergewicht. Der NOAEL-Wert (no adverse effect level) bei subakuter Verabreichung beträgt um $9 \mathrm{mg} / \mathrm{kg} \times \mathrm{d}$. Für die zwischen 4-8 g schweren adulten Zwergfledermäuse errechnet sich aus diesen Daten, daß ab einer täglichen oralen Aufnahme von Chrom-VI-Verbindungen in einer 
Größenordnung um 36-72 $\mu \mathrm{g}$ schädigende Auswirkungen auf das Tier bestehen, die einmalige orale Aufnahme von mehr als etwa $400 \mu \mathrm{g} \mathrm{Cr} r^{6+}$ kann den Tod des Tieres nach sich ziehen.

Tab. 62: Zugelassene Holzschutzmittelgruppen auf der Basis von Metallsalzen mit Angabe der maximal zulässigen Wirkstoffgehalte und der zugelassenen Anwendungsbereiche nach DIN 68 800-T3 04:1990 (BUNDESGESUNDHEITSAMT 1986)

\begin{tabular}{|c|c|c|c|}
\hline "Bezeichnung & Wirkstoff(e) & " max. Wirkstoffgehalt & Anwendungsbereich \\
\hline CF-Salze & $\begin{array}{l}\text { Alkali- oder Silicofluoride } \\
\text { in Verbindung mit Dich- } \\
\text { romaten, Chromtrioxid } \\
\text { oder Chromsäure }\end{array}$ & $\begin{array}{l}\text { Cr: } 25 \% \\
\text { HF: } 17 \% \\
\text { Si;F: } 25 \%\end{array}$ & (GK 1), GK 2, GK 3 \\
\hline CFB-Salze & $\begin{array}{l}\text { Dichromate in Verbindung } \\
\text { mit Alkalifluoriden, Bor- } \\
\text { säure und Borfluoriden }\end{array}$ & $\begin{array}{r}\text { Cr: } 31 \% \\
\text { F: } 29 \% \\
\text { B: } 20 \%\end{array}$ & (GK 1), GK 2, GK 3 \\
\hline CKB-Salze & $\begin{array}{l}\text { Dichromate oder Chrom- } \\
\text { säure mit Kupfersulfat und } \\
\text { Borsäure }\end{array}$ & $\begin{array}{r}\text { Cr: } 36 \% \\
\text { Cu: } 30 \% \\
\text { B: } 21 \%\end{array}$ & (GK 1), GK 2, GK 3, GK 4 \\
\hline CKF-Salze & $\begin{array}{l}\text { Ammoniumdichromat und } \\
\text { Kupferhexafluorsilikat, sel- } \\
\text { tener Kupfersulfat und Al- } \\
\text { kalifluoride }\end{array}$ & $\begin{array}{r}\text { Cr: } 64 \% \\
\text { CuF: } 36 \%\end{array}$ & (GK 1), GK 2, GK 3, GK 4 \\
\hline CK-Salze & $\begin{array}{l}\text { Dichromate oder Chrom- } \\
\text { säure und Kupfersulfat }\end{array}$ & $\begin{array}{l}\text { Cr: } 52 \% \\
\text { Cu: } 46 \%\end{array}$ & (GK 1), GK 2, GK 3, GK 4 \\
\hline
\end{tabular}

GK 1 = Innenbauteile unter ständig trockenen Bedingungen, nicht zur großflächigen Anwendung

GK 2 = Innenbauteile, Außenteile unter Dach, nur vorübergehend befeuchtet

GK $3=$ Witterungsexponierte Bauteile, Innenbauteile mit hoher Feuchtigkeitsbeanspruchung

GK 4 = Bauteile mit ständigem Kontakt mit Erde und/oder Wasser

Für Kupfer gilt ein NOAEL-Wert von $11 \mathrm{mg} / \mathrm{kg}$ x d (KALBERLAH 1999); zuverlässige Daten zur $\mathrm{LD}_{50}$-Dosis bei Kleinsäugern liegen für Kupfer nicht vor.

Bedingt durch die Verhaltensweise (Nutzung von Nischen zwischen Hölzern oder Holz- und Mauerwerk, selten freies Hängen auf dem Dachboden) der Hausfledermäuse, zu denen auch die Zwergfledermäuse zählen, ist ein intensiver Kontakt der Felloberfläche mit dem Holz des Dachstuhles möglich. Besonders bei frisch imprägnierten Hölzern ist damit auch eine Verunreinigung der Felloberfläche möglich. Die Aufnahme der Wirkstoffe aus den Holzbehandlungsmitteln erfolgt überwiegend bei der Fellpflege oder über die Haut (BRAUn 1986, NAGEL 1998).

Zur Prüfung auf eine mögliche Belastung der Tiere durch holzschutzmittelrelevante Schwermetalle wurden Daten aller untersuchten Haarproben der Zwergfledermäuse auf das Auftreten stark erhöhter Chrom- und Kupfergehalte untersucht. Als „stark erhöht" wurden Meßwerte oberhalb des 90. Perzentils definiert. Aufgrund der häufigen 
gemeinsamen Verwendung von Chrom und Kupfer in den salzhaltigen Holzschutzmitteln (vgl. Tab. 62) war zu erwarten, daß bei holzschutzmittelbelasteten Tieren die Häufigkeit des gemeinsamen Auftretens von Chrom- und Kupferwerten oberhalb der 90. Perzentilgrenze größer sein würde als bei nicht exponierten Tieren. Die Anzahl der Meßwerte, die oberhalb der Ausschlußgrenze lagen, war für alle untersuchten Schwermetalle annähernd gleich und betrug 7-8 Tiere von 93 ausgewerteten Tieren.

Die kupferauffälligen Tiere waren jedoch bis auf eine Ausnahme (Tier Nr. 91021) nicht identisch mit den chromauffälligen Tieren (Tab. 63). Die chromauffälligen Tiere Nr. 98060 und eingeschränkt Nr. 98019 wiesen zugleich deutlich höhere Blei- und Zinkgehalte im Haar auf. Ein ebenfalls erhöhter Nickelgehalt war bei den chromauffälligen Tieren trotz der geogen engen Vergesellschaftung von Chrom und Nickel in Mineralien nicht vorhanden. Eine exogene Verunreinigung des Felles der Tiere durch Boden- bzw. Mineralpartikel scheidet somit weitgehend aus.

Tab. 63: Chrom- und kupferauffällige Zwergfledermäuse ( $P$. pipistrellus), deren Haargehalte an Chrom und Kupfer oberhalb des 90. Perzentils lagen $(\mathrm{n}=93)$

\begin{tabular}{ccccl}
\hline \hline Tier Nr. & $\begin{array}{c}\text { Chromgehalt } \\
\text { [ } \boldsymbol{\mu g} \mathbf{g} \mathbf{g} \text { TS] }\end{array}$ & Genus & Alter & \multicolumn{1}{c}{ Herkunft } \\
\hline 98060 & 20 & $\mathrm{~m}$ & subad. & Stadt Osterode a.H. \\
98019 & 17 & $\mathrm{~m}$ & subad. & Bad Sachsa, LK Osterode a.H. \\
94039 & 16 & $\mathrm{~m}$ & ad. & Varmissen, LK Göttingen \\
98022 & 11 & $\mathrm{~m}$ & ad. & Förste, LK Osterode a.H \\
94034 & 9,4 & $\mathrm{~m}$ & ad. & Elbingerode, LK Osterode a.H. \\
91021 & 8,6 & $\mathrm{~m}$ & juv. & Nienstedt, LK Osterode a.H. \\
98014 & 7,4 & $\mathrm{w}$ & ad. & Barbis, LK Osterode a.H. \\
\hline \hline
\end{tabular}

\begin{tabular}{ccccl}
\hline \hline Tier Nr. & $\begin{array}{c}\text { Kupfergehalt } \\
\text { [ } \mathbf{\mu g} / \mathbf{g} \text { TS] }\end{array}$ & Genus & Alter & \multicolumn{1}{c}{ Herkunft } \\
\hline 98044 & 180 & $\mathrm{~m}$ & ad. & Stadt Osterode a.H. \\
88015 & 150 & $\mathrm{w}$ & juv. & Wunstorf \\
96028 & 140 & $?$ & subad. & Bothel, LK Rothenburg/Wümme \\
90005 & 130 & $\mathrm{~m}$ & ad. & Bleckede, LK Lüneburg \\
91019 & 83 & $\mathrm{~m}$ & juv. & Lüneburg, Kirche \\
91021 & 83 & $\mathrm{~m}$ & juv. & Nienstedt, LK Osterode a.H. \\
96023 & 59 & w & subad. & Rhumspringe, LK Göttingen \\
\hline \hline
\end{tabular}

Aufgrund der annähernd gleichverteilten Häufigkeit des Auftretens von Meßwerten oberhalb des 90 . Perzentils für alle untersuchten Schwermetalle sowie der nicht erkennbaren Korrelation des Chrom- und Kupfergehaltes bei den untersuchten Fledermäusen kann eine besondere Belastung der Hausfledermäuse durch salzhaltige, wasserlösliche Holzschutzmittel auf der Basis von Chrom- und Kupfersalzen nicht nachgewiesen werden. 


\subsection{Auswirkungen erhöhter Schwermetallbelastungen der Umwelt auf die Bestandsentwicklung der Fledermäuse}

Die in der vorliegenden Untersuchung nachgewiesene Abhängigkeit des Schwermetallgehaltes der Fledermäuse von der Belastungssituation der Umwelt läßt die Frage nach den Auswirkungen auf die Bestandsentwicklung der Tiere aufkommen. Grundsätzlich ist zunächst festzustellen, daß die vorliegenden Daten in keinem Falle Hinweise auf eine akute Schwermetallintoxikation geben, die zum Tode eines Tieres hätte führen können. In der Literatur finden sich jedoch Hinweise, daß speziell eine erhöhte Inkorporation von Blei bereits im subtoxischen Bereich zu nachweisbaren Veränderungen speziell des Nervensystemes führen. LILIENTHAL (1992) untersuchte am Primatenmodell die Wirkung subtoxischer Bleibelastungen auf akustisch evozierte Hirnstammpotentiale. Hierbei konnte er eine deutliche Reduzierung der Nervenleitgeschwindigkeit bei den bleiexponierten Versuchstieren nachweisen. Nach Absetzen der „Bleidiät“ ergaben sich dabei keine Anzeichen für eine Regeneration. Gleichwohl konnten aber additive Effekte bei mehrfacher Bleiinkorporation nachgewiesen werden. LILIENTHAL betont unter Bezugnahme auf publizierte Daten zahlreicher Laborversuche mit anderen Säugetieren, daß eine der hervorstechenden Eigenschaften des akustisch evozierten Hirnstammpotentiales seine große Ähnlichkeit im interspezifischen Vergleich zumindest in der Klasse der Säugetiere ist. Dies bedeutet, die auftretenden langsameren Nervenleitgeschwindigkeiten sind auch für bleibelastete Fledermäuse zu erwarten.

Zu ähnlichen Ergebnissen kam die Studie von BRUCKS (1998). Die Autorin untersuchte die mögliche, subklinisch neurotoxische Schädigung durch Blei an langjährig exponierten Arbeitern einer Kupferhütte bei Hettstedt am östlichen Harzrand. Es ergaben sich bei den bleiexponierten Arbeitern psychometrisch erfaßbare Leistungsdefizite in der Motorik. So benötigten die bleiexponierten Probanden hoch signifikant länger für die Beantwortung (per Knopfdruck) auf ein dargebotenes Lichtsignal. Von der Gruppe der bleiexponierten Probanden wurden zudem wesentlich häufiger Symptome wie körperliche und geistige Ermüdung, Energiemangel, Konzentrationsschwierigkeiten und Vergeßlichkeit beschrieben.

Untersuchungen von SCHWARTZ et al. (2000) an 535 ehemaligen Beschäftigten der Organobleiherstellung belegen, daß auch lange Zeit nach der eigentlichen Bleiexposition noch neurotoxische Effekte auftreten bzw. sich nicht zurückbilden. So nahm das cognitive Vermögen der vor rund 16 Jahren letztmalig bleiexponierten Personengruppe im Vergleich zu einer aus 181 Personen bestehenden Kontrollgruppe aus der Nachbarschaft der Bleiarbeiter signifikant schneller ab als bei der Kontrollgruppe.

Bei dem Versuch, die Untersuchungsergebnisse von LILIENTHAL (1992) und BRUCKS (1998) auf die Ordnung der Fledermäuse zu übertragen, kommen den spezifischen Besonderheiten der Lebensweise und des Nahrungserwerbs bei den mitteleuropäischen Fledermausarten besondere Bedeutung zu. Fledermäuse orten ihre Beutetiere im Fluge mit Ultraschall, wobei über den Laufzeitunterschied die Entfernung zum Beutetier ermittelt und aus der Differenz der eintreffenden Schallsignale am linken 
und rechten Ohr sowie über Frequenzunterschiede die Lage des Beuteobjektes im Raum bestimmt wird (NEUWEILER 1993). Somit sind Fledermäuse in besonderer Weise auf die Leistungsfähigkeit des Nervensystemes und im besonderen des akustisch evozierten Hirnstammpotentiales angewiesen. Wenn, wie zu vermuten ist, erhöhte inkorporierte Bleigehalte auch bei Fledermäusen eine Reduzierung der Leistungsfähigkeit des Nervensystemes bewirken, dann vermindert sich hierdurch der Jagderfolg der Fledermäuse im Vergleich zu anderen Säugetieren in besonderer Weise. Eine Verminderung des Jagderfolges bedeutet einen erhöhten Energieaufwand für die Tiere bei der Nahrungsbeschaffung. Die vom Jagderfolg der Muttertiere abhängigen Jungtiere sind so mit höherer Wahrscheinlichkeit dem Verhungern ausgesetzt, woraus mittelfristig ein Bestandsrückgang resultiert.

Die Untersuchungen an den Fledermaustotfunden aus Niedersachsen ergaben signifikant höhere Bleibelastungen der Fledermäuse in den achtziger Jahren des 20. Jahrhunderts als gegen Ende der neunziger Jahre des 20. Jahrhunderts. Hauptursache der erhöhten Bleibelastungen der Tiere war die Verwendung von verbleiten Kraftstoffen, wie aus der globalen Abnahme der Bleibelastung in allen Umweltkompartimenten nach Umsetzung der letzten Stufe des Benzin-Bleigesetzes erkennbar ist.

Aufgrund der Ergebnisse der vorliegenden Schwermetallrückstandsanalysen an Fledermäusen und den neueren neurotoxikologischen Studien zu Auswirkungen einer früheren Bleiexposition kann nach Einschätzung des Autors davon ausgegangen werden, daß der seit etwa Mitte des 20. Jahrhunderts beobachtete Bestandsrückgang der Fledermäuse auch durch den steigenden Bleigehalt in allen Umweltkompartimenten mit verursacht worden ist.

Organochlorpestiziden, die z.T. in hoher Konzentration im Fettgewebe der Tiere nachgewiesen wurden (Nagel 1998), kommt eine weitere wichtige Bedeutung bei dem festgestellten Bestandsrückgang zu. Den häufig als Grund für den Bestandsrückgang angeführten Zerstörungen von Sommer- und Winterquartieren, z.B. durch Wärmedämm-Maßnahmen und Altstadtsanierungen, wird hingegen weniger Bedeutung beigemessen. Wenn dies eine der Hauptursachen für die negative Bestandsentwicklung wäre, dann hätte zunächst der Besiedelungsdruck auf die verbliebenen Quartiere steigen müssen. Dies war jedoch nicht der Fall, vielmehr ging in allen Quartieren die Populationsdichte bis etwa in die neunziger Jahre des 20. Jahrhunderts zurück. Die gegenwärtig zu beobachtende leichte Bestandserholung tritt ein, obwohl das Quartierangebot nicht wesentlich zugenommen hat (BOYE et al. 1999, RACKOW mdl. Mitt.).

Eine Absicherung dieser Hypothese kann nur durch Tierexperimente mit Fledermäusen, denen eine spezielle Bleidiät verabreicht wurde, erfolgen. Aufgrund der noch immer geringen Bestandsdichte der einheimischen Fledermäuse bieten sich hierfür allenfalls tropische, carnivore Fledermausarten aus großen Populationen an. 


\subsection{Platingruppenelemente in Umweltproben}

Mit der Umstellung auf bleifreie Vergaserkraftstoffe nahm ab 1984 der Einsatz von Abgaskatalysatoren für Kraftfahrzeuge zu. Stand der Technik ist der sogenannte Drei-Wege-Katalysator, der die im Abgas enthaltenen Bestandteile Kohlenmonoxid, Kohlenwasserstoffe und Stickoxide überwiegend in die unschädlicheren Bestandteile Kohlendioxid, Wasser und Stickstoff zerlegt. Die Abgaskatalysatoren bestehen aus einem keramischen Material als Träger der eigentlichen Katalysatormetalle sowie zur Vergrößerung der inneren Oberfläche des Katalysators. Beimengungen von Cer dienen der Erhöhung der Reaktionsgeschwindigkeit. Zur Steuerung der eigentlichen Oxidations- und Reduktionsprozesse im Katalysator enthalten diese bis zu drei Gramm der Edelmetalle Platin, Palladium und Rhodium. Dieser vergleichsweise hohe Edelmetallanteil führt dazu, daß gegenwärtig rund $90 \%$ der Weltproduktion an diesen Metallen in die Katalysatorindustrie gehen (ECKHARDT und SCHÄFER 1997).

Mit der Verwendung der Platingruppenelemente (PGE) gelangen diese auch über das Abgas in die Umwelt, wobei ein feinpartikulärer Austrag die häufigste Art der Emission darstellen dürfte. Über das Verhalten der Platingruppenelemente in der Umwelt ist wenig bekannt, das Verteilungsmuster dürfte jedoch identisch mit dem der anderen Kraftfahrzeugemissionen sein. ZEREINI et al. (1997a) errechneten aus Bodenanalysen entlang der Bundesautobahn A 67 Frankfurt-Mannheim eine Platinemission je Katalysator von rund $270 \mathrm{ng} / \mathrm{km}$. Im Prüfstandversuch wurden nach ZeREINI et al. (1997a) lediglich 2-78 ng/km Platin emittiert.

Entlang der Hauptverkehrsstraßen ist bereits eine signifikante Platinanreicherung und eine Anreicherung der anderen Platingruppenelemente wie Palladium und Rhodium, aber auch für Cer sowie Indium nachgewiesen worden (ZEREINI et al. 1997a, b, 1998, GolWER und ZerEINI 1998, ZeREINI und ALt 1999, 2000). Die durchschnittlichen Platingehalte am Fahrbahnrand der A 66 betrugen nach ZEREINI et al. (1993) um $28 \mu \mathrm{g} / \mathrm{kg}$ TS und sinken mit zunehmender Entfernung vom Fahrbahnrand. In 15 $\mathrm{m}$ Entfernung waren nur noch Gehalte um $5 \mu \mathrm{g} / \mathrm{kg}$ TS nachweisbar. Der geogene Backgroundgehalt beträgt nach ECKHARDT und SCHÄFER (1997) unter 0,8 $\mu \mathrm{g} / \mathrm{kg}$ TS.

Neben der Anreicherung von Platin im Boden erfolgt auch eine Ablagerung dieser Metalle auf Pflanzen. HeLmers und Mergel (1997) zeigten im Rahmen einer Untersuchung in den Jahren 1992-1995, also in einem Zeitraum von etwa zehn Jahren nach Einführung der Kraftfahrzeugkatalysatoren, daß in unmittelbarer Nähe der Fahrbahn Grasproben einen achtmal höheren Platingehalt aufwiesen als in etwa 10 m Entfernung zur Fahrbahn.

Die vorliegenden, an Fledermauskot und Fledermaushaaren gewonnenen Analysenergebnisse können aufgrund der geringen Probenanzahl nur als orientierende Messungen beurteilt werden und erlauben keine Aussage zu den durchschnittlichen 
Platingehalten in dem Probenmaterial. Diese Meßwerte zeigen lediglich, daß das Katalysatormaterial Platin auch bereits in biologischen Proben von Fledermäusen nachweisbar ist.

Über das ökotoxikologische Verhalten von Platin und Platingruppenelementen in der Umwelt ist bislang kaum etwas bekannt. Gleichfalls unbekannt sind mögliche Reaktionen von Platin und Platingruppenelementen in der Bodenlösung, z.B. unter sauren Bedingungen und hohen Salzkonzentrationen, wie sie entlang der Hauptverkehrswege typisch sind.

Bei der Tumorbehandlung werden cis-Platin und Carboplatin als Zytostatica verwendet. KALDEN et al. (1997) untersuchten das Haar von 16 Patienten, die sich in einer zytostatischen Behandlung mit platinhaltigen Medikamenten befanden, und eine aus 20 Personen bestehende Kontrollgruppe. Sie stellten fest, daß die Platinkonzentration im Blut sehr rasch wieder abnimmt. Haare, die nach Beendigung der Platintherapie entnommen wurden, wiesen etwa gleichhohe Platingehalte auf wie die Haare der Kontrollgruppe. Nur während der zytostatischen Behandlung konnten maximal $0,87 \mu \mathrm{g} / \mathrm{g}$ Platin im Haar gemessen werden. Im Körpergewebe reichert sich Platin vor allem in Haut, Nieren, Uterus, Leber, Ovar, Muskeln und Hoden an (LITTERST et al. 1979). Die von KRAUSE et al. (1997) im Rahmen des bundesweiten Umweltmonitorings erhobenen Daten zum Platingehalt im Haar betragen im Mittel 3,9 ng/g. Die für das Haar der Zwergfledermäuse erhobenen Daten entsprechen etwa der gleichen Größenordnung.

Der fehlende Nachweis von Platin in den untersuchten Knochenproben der Fledermäuse dürfte primär im Zusammenhang mit dem nicht vorhandenen guten Bindungsvermögen zum Phosphat des Knochengewebes zu sehen sein. Anders als Blei, welches das Calcium aus seiner Bindung mit dem Phosphat teilweise verdrängt und damit im Knochen als Knochenbaustoff eingelagert wird, reagiert Platin nicht mit der Knochenmatrix. Die zytostatische Wirkung beruht vielmehr auf Plasmaproteinbindungen des cis-Platins.

Aus Gründen des vorbeugenden Umweltschutzes sollten Kinetik und Verteilung der Platingruppenelemente in den verschiedenen Umweltkompartimenten besondere Beachtung finden. Solange mögliche Reaktionen der PGE-Emissionen in der Bodenlösung und mögliche (zytostatische) Auswirkungen auf die Bodenfauna und die Bodenmikrofauna nicht näher untersucht sind, können langfristige nachteilige Umweltauswirkungen nicht ausgeschlossen werden.

\section{Zusammenfassung}

Im Rahmen der vorliegenden Untersuchung wurde der Schwermetallgehalt in Organen (Lunge, Leber, Nieren), im Unterarmknochen, im Haar von Fledermäusen sowie 
am Kot bestimmt. Den Untersuchungen lagen für die Knochenuntersuchungen insgesamt Stichprobengrößen von 30 Großen Mausohren (Myotis myotis) sowie 111 Zwergfledermäusen (Pipistrellus pipistrellus) zugrunde. An 95 Zwergfledermäusen erfolgte die Schwermetallbestimmung der Haare. Organe wurden von 22 Zwergfledermäusen analysiert. Ergänzend wurden 48 Kotproben aus verschiedenen Quartieren untersucht. Das Tiermaterial entstammt Totfunden aus den Jahren 1987 bis 1999, die in der Tiermedizinischen Hochschule der Universität Hannover durch Tiefgefrieren im Auftrage des Niedersächsischen Landesamtes für Ökologie konserviert worden waren. Erstmalig erfolgte auch eine vollständige Erfassung des dort konservierten Materiales. Insgesamt wurden 458 Individuen aus 13 Artengruppen erfaßt. Zwergfledermäuse waren mit rund $55 \%$ Anteil an den Totfunden die dominante Art. Dies wird jedoch nur bedingt auf die größere Häufigkeit des Vorkommens der Zwergfledermaus im Vergleich zu anderen Arten zurückgeführt. Wesentlich ist auch der Lebensraum der Tiere, der entscheidend für einen Totfund ist. Die sogenannten Hausfledermäuse, die bevorzugt im Umfeld menschlicher Siedlungen leben, können weitaus einfacher entdeckt werden als tote Tiere in der offenen Landschaft, die zudem rasch einem Aasfresser als Nahrung dienen.

Aufgrund jüngster Veröffentlichungen zur Differenzierung der unterschiedliche Ruffrequenzen aufweisenden Zwergfledermausgruppen erfolgte zusätzlich eine Auswertung biometrischer Arten von 71 Zwergfledermäusen. Es ergab sich dabei kein gesicherter Nachweis der als Mückenfledermaus bezeichneten, bei $55 \mathrm{kHz}$ rufenden Variante. Die Untersuchungen ergaben vielmehr, daß bei Jungtieren die zur Artdifferenzierung der Mückenfledermaus von der eigentlichen Zwergfledermaus verwendeten Merkmale (Fingerlied II gleichlang Fingerglied III am 3. Finger) nicht zutreffend sind. Bei nicht ausgewachsenen Tieren beträgt das Längenverhältnis immer um 1.

Zur Erfassung der endogenen Bleibelastung der Tiere während der gesamten Wachstumsphase erfolgte die Präparation und Analyse des Unterarmknochen. Über die Haaruntersuchung wurden Ergebnisse der Schwermetallinkorporation während der letzten Lebensmonate der Tiere gewonnen.

Die Analysen erfolgten überwiegend mittels Atomabsorptionsspektralphotometrie (AAS). Kotproben wurden auch mittels ICP-OES untersucht. Obwohl überwiegend nur geringe Probenmengen von weniger als $30 \mathrm{mg}$ zur Verfügung standen, ergaben Wiederholungsmessungen an Knochenproben nur geringe Abweichungen, die im Mittel 8,5\% betrugen.

Für den Bleigehalt im Knochen der untersuchten Tiere zeigte sich für nicht bleibelastete Regionen ein Rückgang der Bleigehalte über den gesamten Betrachtungszeitraum von 1987 bis 1999 um rund 50 \%. Der ermittelte Rückgang der Bleibelastung ist im wesentlichen auf die Einführung von bleifreien Kraftstoffen zurückzuführen. Untergeordnet sind auch verbesserte Abluftreinigungstechniken der Industrie von Bedeutung. 
Fledermäuse aus Regionen mit erhöhten Schwermetallgehalten im Boden (Westharz), wiesen signifikant höhere Bleigehalte im Knochen auf als Fledermäuse, die durchschnittlich belasteten Regionen entstammen. Der Quartilsbereich der aktuellen Knochenbleikonzentration der Zwergfledermaus für nicht vorbelastete Regionen beträgt 7-17 $\mu \mathrm{g} / \mathrm{g}$ TS. Für den Bereich der geogen erhöht bleibelasteten Region des Westharzes betragen die Quartilswerte der Knochenbleikonzentration hingegen 8,8$40 \mu \mathrm{g} / \mathrm{g}$ TS. Sie liegen damit gegenwärtig noch immer in dem Konzentrationsbereich, der für alle Fledermäuse um 1987 gegen Ende der Verwendung von verbleiten Kraftstoffen typisch war.

Der Bleigehalt im Knochengewebe ist dabei abhängig vom Alter der Tiere. Geschlechtsspezifische Unterschiede im Bleigehalt der Unterarmknochen der Zwergfledermaus waren nicht nachweisbar. Im Vergleich zum Großen Mausohr liegt der Medianwert des Knochenbleigehaltes bei der Zwergfledermaus um rund $70 \%$ höher.

Neben der Region Harz fanden sich erhöhte Bleigehalte in Tieren aus dem Bereich Greene bei Northeim. Die hier vorkommenden Zwergfledermäuse und Großen Mausohren wiesen sowohl im Knochengewebe wie im Haar auffallend hohe Bleigehalte auf. Die Ursache dieser hohen Belastung ist gegenwärtig noch unbekannt und bedarf einer näheren Untersuchung des Umfeldes dieser Quartiere.

In den untersuchten Organen zeigte sich gleichfalls für die Tiere aus dem Harz ein signifikant erhöhter Bleigehalt in der Leber $(p=0,057)$ und in den Nieren $(p=0,002)$. Während der Medianwert für Blei in der Leber bzw. in den Nieren von Tieren aus dem Bereich des Harzes 6,3 bzw. 2,7 $\mu \mathrm{g} / \mathrm{g}$ TS betrug, lag der Medianwert für die untersuchten Tieren aus den übrigen niedersächsischen Regionen bei 2,0 (Leber) bzw. $1,2 \mu \mathrm{g} / \mathrm{g}$ TS (Nieren). Kein Unterschied im Bleigehalt zeigte sich hingegen beim Vergleich der Lungengewebeproben von Tieren aus dem Harz und aus harzfernen Regionen. Die inhalative Aufnahme von schwermetallhaltigen Stäuben dürfte somit nicht den Hauptpfad der Schwermetallaufnahme darstellen. Die Schwermetallaufnahme erfolgt nach den vorliegenden Untersuchungen primär mit der Nahrung. Für die ebenfalls im Leber- und Nierengewebe analysierten Elemente Cadmium und Chrom konnte kein regionaler Unterschied nachgewiesen werden.

Ähnliche Unterschiede ergaben sich für die durchgeführten Haaranalysen, wenngleich hier der Unterschied weniger deutlich ausfällt. Über den gesamten Betrachtungszeitraum (1987-1999) nahm der Medianwert des Haarbleigehaltes von $56 \mu \mathrm{g} / \mathrm{g}$ auf $23 \mu \mathrm{g} / \mathrm{g} \mathrm{ab}$, dies bedeutet ein Rückgang um rund $55 \%$. Signifikante Unterschiede der Haarbleibelastung zwischen der Region des Harzes und dem übrigen Niedersachsen waren nicht nachweisbar. Zurückgeführt werden kann dies auf die fehlende spezifische Bleianreicherung in der Haarmatrix. Die bevorzugte Einlagerung und Anreicherung von Blei erfolgt im Knochengewebe. Nur bei stark erhöhtem Blutbleispiegel ist auch ein nachweisbarer Effekt im Haar zu erwarten. Abnehmende Gehalte im Haar über den gesamten Betrachtungszeitraum konnten ferner für die Elemente 
Cadmium (rund 40-50 \% Rückgang) und Nickel (rund $10 \%$ Rückgang) nachgewiesen werden. Praktisch unverändert über den gesamten Betrachtungszeitraum waren die Elementgehalte für Chrom, Kupfer und Zink im Haar. Zumindest Kupfer und Zink unterliegen im Säugetierorganismus einer homöostatischen Regulation. Dieser Mechanismus dürfte auch für den einheitlichen Verlauf der Kupfer- und Zinkgehalte im Haar verantwortlich sein. Regionale oder geschlechtsspezifische Unterschiede fanden sich für die untersuchten Schwermetalle nicht.

Ein Rückgang der Bleigehalte konnte gleichfalls in horizontiert untersuchten Bohrkernen aus zwei Kothaufen des Großen Mausohres bei Ahlden und Steinbergen nachgewiesen werden. Proben aus einem Zeitraum um 1990 wiesen mit 12 bis $15 \mathrm{mg} / \mathrm{kg}$ TS Blei einen rund 50-60 \% höheren Bleigehalt auf als die aus dem Jahre 1999 stammenden Kotproben derselben Quartiere (6,5 bzw. 6.2 mg/kg TS). Horizontiert untersuchte Proben aus einer jetzt erloschenen Wochenstube des Großen Mausohres ergaben zwischen 1980 und 1986, also während eines Zeitabschnittes, in dem fast ausschließlich verbleite Kraftstoffe verwendet wurden, hingegen einen Anstieg der Bleigehalte. Für die übrigen untersuchten Schwermetalle ergaben sich keine wesentlichen Unterschiede.

Auf der Grundlage der vorliegenden Untersuchungen kann für die Zwergfledermäuse aus nicht geogen oder anthropogen vorbelasteten Regionen Niedersachsens im Zeitraum 1987 bis 1999 insgesamt ein Rückgang der Bleibelastung um rund 50 \% festgestellt werden.

Stichprobenartige Untersuchungen auf Platin, einem typischen, mit Einführung der Abgaskatalysatoren in Kraftfahrzeugen in die Umwelt emittierten Element, ergaben meßbare Gehalte sowohl im Kot der Fledermäuse als auch im Haar der Tiere. So fand sich Platin im Kot in einer maximalen Konzentration von 6,8 ng/g TS. Im Haar konnte ein maximaler Platingehalt von 2,6 $\mathrm{ng} / \mathrm{g}$ TS ermittelt werden. Nicht bzw. an der Bestimmungsgrenze $(<0,5 \mathrm{ng} / \mathrm{g}$ TS) lag hingegen der Platingehalt in den untersuchten Unterarmknochen. Aussagen zu möglichen ökotoxikologischen Langzeitwirkungen dieser Edelmetallemissionen sind gegenwärtig aufgrund des sehr geringen Forschungsstandes nicht möglich.

Bei den Fledermaushaaren fällt im Vergleich zu Humanhaar der große Unterschied beim Blei auf. Während die übrigen an den Haarproben von Fledermaushaaren analysierten Schwermetalle eine gute Übereinstimmung zu Daten von Humanhaaruntersuchungen zeigen, liegen die Haarbleigehalte bei der Zwergfledermaus deutlich höher. Die Ursache hierfür wird primär in dem standortbezogenen Nahrungserwerb der Fledermäuse im Vergleich zum Menschen gesehen.

Auf der Grundlage der vorliegenden Meßwerte werden Referenzbereiche der zu erwartenden Schwermetallgehalte im Fledermauskot, in Organen, im Knochen und im Haar der Tiere abgeleitet. Die Referenzbereiche wurden ausschließlich auf der Basis der Untersuchungen der Zwergfledermäuse errechnet. Die Übertragbarkeit dieser 
Werte auf andere Fledermausarten bedarf der näheren Einzelfallprüfung. Der Vergleich mit Literaturangaben zeigt aber, daß zur groben Orientierung und ersten Abschätzung einer möglichen signifikanten Schwermetallbelastung eines Tieres diese Werte auch für andere Arten herangezogen werden können. Die errechneten Referenzwerte betragen für die einzelnen Organe und Gewebe wie folgt:

Knochen:

Blei: $\quad 7-17 \quad \mu g / g$ TS

Lunge:

Blei: $\quad 0,5-0,9 \quad \mu g / g$ TS

Leber:

Blei: $\quad 1,2-4,7 \quad \mu g / g$ TS

Cadmium : $0,05-1,4 \quad \mu \mathrm{g} / \mathrm{g}$ TS

Chrom, ges.: 0,02-0,05 $\mu \mathrm{g} / \mathrm{g}$ TS

Nieren:

Blei: $\quad 0,6-1,5 \quad \mu g / g$ TS

Cadmium : $0,2-2,7 \quad \mu \mathrm{g} / \mathrm{g}$ TS

Chrom, ges.: 0,02-0,2 $\mu \mathrm{g} / \mathrm{g}$ TS

Haare (mit $50 \%$ Feuchtegehalt):

Blei: $\quad 8-52 \quad \mu \mathrm{g} / \mathrm{g}$

Cadmium: $\quad 0,15-0,55 \mu \mathrm{g} / \mathrm{g}$

Chrom, ges.: 1,2 - 3,5 $\quad \mu \mathrm{g} / \mathrm{g}$

Kupfer: $\quad 13-36 \quad \mu \mathrm{g} / \mathrm{g}$

Nickel: $\quad 1,2-2,6 \quad \mu \mathrm{g} / \mathrm{g}$

Zink: $\quad 230-390 \mu \mathrm{g} / \mathrm{g}$

Kot:

Arsen, ges.: $2,1-5,2 \quad \mathrm{mg} / \mathrm{kg}$ TS

Blei: $\quad 3,2-7,7 \quad \mathrm{mg} / \mathrm{kg}$ TS

Cadmium: $\quad 1,6-3,2 \quad \mathrm{mg} / \mathrm{kg}$ TS

Chrom, ges.: 1,6 - 3,6 mg/kg TS

Kupfer: $\quad 77-120 \quad \mathrm{mg} / \mathrm{kg}$ TS

Nickel: $\quad 1,5-3,5 \quad \mathrm{mg} / \mathrm{kg}$ TS

Zink: $\quad 460-900 \quad \mathrm{mg} / \mathrm{kg}$ TS

Neurophysiologische Studien am Menschen und an Primaten zeigen einen meßbaren Zusammenhang zwischen subtoxischer Bleibelastung des Organismus und der Leistungsfähigkeit des Nervensystemes. In Abhängigkeit vom Bleigehalt im Körper veränderten sich akustisch evozierte Hirnstammpotentiale bei Primaten, und es nahm das Reaktionsvermögen sowie das cognitive Vermögen signifikant ab. Auch lange Zeit nach Beendigung der Bleiexposition dauerte dieser Zustand an. 
Die vorliegenden Untersuchungsergebnisse belegen, mit Ausnahme geogen bzw. anthropogen vorbelasteter Regionen, einen deutlichen Rückgang der Bleibelastung bei Fledermäusen. Unter Berücksichtigung der an Primaten und am Menschen gewonnenen neurophysiologischen Daten zu den Auswirkungen erhöhter Bleibelastungen wird versucht, dies auf die Ordnung der Fledermäuse zu übertragen. Hierbei kommen den spezifischen Besonderheiten der Lebensweise und des Nahrungserwerbs der Fledermäuse besondere Bedeutung zu. Fledermäuse sind in besonderer Weise auf die Leistungsfähigkeit des Nervensystemes angewiesen. Die mitteleuropäischen Fledermausarten orten ihre Beutetiere im Fluge mit Ultraschall, wobei über den Laufzeitunterschied die Entfernung zum Beutetier ermittelt und aus der Differenz der eintreffenden Schallsignale am linken und rechten Ohr sowie über Frequenzunterschiede die Lage des Beuteobjektes im Raum bestimmt wird.

Wenn, wie zu vermuten ist, erhöhte inkorporierte Bleigehalte auch bei Fledermäusen eine Reduzierung der Leistungsfähigkeit des Nervensystemes bewirken, dann vermindert sich hierdurch der Jagderfolg der Fledermäuse im Vergleich zu anderen Säugetieren in besonderer Weise. Eine Verminderung des Jagderfolges bedeutet einen erhöhten Energieaufwand für die Tiere bei der Nahrungsbeschaffung. Die vom Jagderfolg der Muttertiere abhängigen Jungtiere sind so mit höherer Wahrscheinlichkeit dem Verhungern ausgesetzt, woraus mittelfristig ein Bestandsrückgang resultiert. Dies wäre, neben der Belastung der Umwelt durch Pestizide, eine Erklärung, warum mit der Intensivierung des Kraftfahrzeugverkehres seit Mitte des 20. Jahrhunderts ein kontinuierlicher Bestandsrückgang der Fledermäuse zu beobachten war und nach Einstellung der Verwendung verbleiter Vergaserkraftstoffe mit leichter zeitlicher Verzögerung nunmehr eine leichte Bestandserholung zu verzeichnen ist. Andere Säugetiere sind von der als ubiquitär zu beurteilenden Bleibelastung der Umwelt infolge der früheren Verwendung verbleiter Kraftstoffe in gleicher Weise betroffen, nur sind die Auswirkungen nicht so gravierend, da kein anderes Säugetier in so hohem Maße wie die Fledermaus bei dem Nahrungserwerb auf die Leistungsfähigkeit gerade des Hörsinnes und des Nervensystemes angewiesen ist.

\section{Literatur}

ALIKE, R. (1974): Die hydrochemischen Verhältnisse im Westharz in ihrer Beziehung zur Geologie und Petrographie. - Clausthaler geol. Abh., 20, 223 S., 32. Abb., Anh. m. Tab., Clausthal-Zellerfeld.

Alvarez-Castaneda S.T., L. Mendez (1998): Comparative Analysis of Heavy Metals in two Species of Ichthyophagous Bats Myotis vivesi and Noctilio leporinus. Rep. of the 45th Annual Meeting of the Southwestern Association of Naturalists, Albuquerque, New Mexico.

ANKE, M. (1993): Die Bedeutung der Spurenelemente für die Fauna. - In: FIEDLER, H.J. und H.J. RÖSLER (Hrsg.): Spurenelemente in der Umwelt. - 221-240, Fischer, Stuttgart. 
ANKE, M. und M. RISCH (1979): Haaranalyse und Spurenelementstatus. - 267 S., 38 Abb., 204 Tab, Fischer, Jena.

ARBEITSGEMEINSCHAFT ZUR REINHALTUNG DER WESER (1996): Schwermetallbelastung von Schwebstoffen und Sediment an Oker und Innerste. - Schriftenreihe der ArGe Weser, 4, 1-44.

BAchmann, G., C.G. BANNIK, E. GIESE et al. (1998): Fachliche Eckpunkte zur Ableitung von Bodenwerten im Rahmen des Bundes-Bodenschutzgesetzes. - 121 S., E. Schmidt, Berlin.

Bachmann, H.-G., Chr. Bartels, A. Bingener und L. Klappauf (2000): Daten zur Geschichte der Harzregion. - In: SEgERS-GLockE, Chr. (Hrsg.): Auf den Spuren einer frühen Industrielandschaft. Arbeitshefte für Denkmalpflege in Niedersachsen, 21, 157-165, Hameln.

BARRY, P.S.I. (1975): A comparison of concentrations of lead in human tissues. British Journal of Industrial Medicine, 32, 119-139.

BARTELS, Chr. (1992): Vom frühzeitlichen Montangewerbe zur Bergbauindustrie: Erzbergbau im Oberharz 1635-1866. - Veröffentlichungen aus dem Deutschen Bergbau-Museum Bochum, 54, 740 S., Bochum.

BARTENS, H. und G. BütTNER (1997): Bodenzustandserhebung in Niedersachsen 1990/92 - Datendokumentation. - Berichte Forschungszentrum Waldökosysteme, C 5, Göttingen.

BAUEROVA, Z. (1978): Contribution to the trophic ecology of Myotis myotis. - Folia Zoologica, 27, 305-316.

BODE, A. (1928): Reste alter Hüttenbetriebe im West- und Mittelharz. - Jb. Geogr. Ges., 141-197, Hannover.

BOYe P., M. DieTZ und M. Weber (1999): Fledermäuse und Fledermausschutz in Deutschland. - 110 S., Bundesamt für Naturschutz, Bonn.

BRAUCKHOFF, H. (1944): Die Zustandsänderung normaler, ausgetrockneter und gedehnter Haare in Abhängigkeit von der relativen Feuchtigkeit. - Biochem. Z., 317, 159-167.

BRAUN, M. (1986): Rückstandsanalysen bei Fledermäusen. - Z. Säugetierkde., 51, 212-217.

BRINGEZU, S. (1992): Konzept für die Prüfung und Bewertung der Umweltverträglichkeit von Holzschutzmitteln. - Texte 13/92, 85 S., Anh., Berlin.

BRUCKS, U.M.C. (1998): Subklinische neurotoxische Effekte bei langjähriger Bleiexposition: eine Felduntersuchung in der Kupfer-Silberhütte-Hettstedt. - 63 S., Diss. Univ. Magdeburg.

BUNDESGESUNDHEITSAMT (1986): Bekanntmachungen des Bundesgesundheitsamtes, Holzschutzmittel 1. Mitteilung. - Bundesgesundheitsblatt, 29, 387-394.

BURKARD, C. (1999): Elementanomalien in Erdfall-Sedimenten als Zeugen der historischen Metallgewinnung im südwestlichen Harzvorland am Beispiel der Beiersteinsenke (Osterode/Harz). - 102 S., 23 Abb., 13 Tab., Dipl.arbeit Univ. Göttingen.

BüTTNER, G. (1997): Ergebnisse der bundesweiten Bodenzustandserhebung im Wald (BZE) in Niedersachsen 1990-1991. - Schriften a.d. Forstlichen Fakultät der Universität Göttingen und der Nieders. Forstl. Versuchsanstalt, 122, Sauerländer, Frankfurt a.M. 
CANNON, J. (1999): Stellaluna. - 24 S., Carlsen, Hamburg.

CLARK, D.R. (1979): Lead concentrations: bats vs. terrestrial small mammals collected near a major highway. - Environm. Sci. Technol., 13, (3), 338-341.

CLARK, D.R. (1981): Bats and Environmental Contaminants: A Review. - USDA Fish Wildl. Serv. Spec. Scient. Rep. Wildlife, 235, 1-27.

Clark, D.R., A.S. Wenner, J.F. MoOre (1986): Metal Residues in Bat Colonies, Jackson Country, Florida 1981-1983. - Florida Field Naturalist, 14, 38-45.

DEICKE, M. und H. RUPPERT (2000): Frühe Metallgewinnung und Umweltbelastungen im Harz - Umweltgeochemische Aspekte. - In: SEgERS-GLOCKE, Chr. (Hrsg.): Auf den Spuren einer frühen Industrielandschaft. Arbeitshefte für Denkmalpflege in Niedersachsen, 21, 78-82, Hameln.

Disser, J., A. NAGEL (1987): Residues of organochlorine Pesticides in a materny group of the Common Pipistrelle. - Fourth european bat research symposium, Prag.

ECKARDT, J.-D. und J. SCHÄFER (1997): PGE-Emissionen aus Kfz-Abgaskatalysatoren. - In: MATSCHULLAT, J., H.J. TOBSCHALL, H.-J. VoIGT (Hrsg.): Geochemie und Umwelt, 181-188, Springer, Berlin, Heidelberg.

EICHSTÄDT, H.; W. BASSUS (1995): Untersuchungen zur Nahrungsökologie der Zwergfledermaus (Pipistrellus pipistrellus). - Nyctalus (N.F.), 5, 561-584.

FiedLeR, H.J. und H.J. RöSlER (1993): Spurenelemente in der Umwelt. - 385 S., Fischer, Stuttgart.

FYTIANOS, K.K. (1978): Untersuchungen auf Schwermetalle in Fließgewässern und Flußsedimenten des West-Harzes. - 134 S., Diss. Göttingen.

GatTERER, C.W.J. (1786): Anleitung den Harz und andere Bergwerke mit Nuzen zu bereisen. - 18+358 S., Göttingen.

Gerell, R., K. Gerell-Lundberg (1993): Decline of a bat Pipistrellus pipistrellus population in an industrialized area in south sweden. - Biol. Conservations, 65, 153-157.

Gibson, R.S., E.F. Ferguson, P.D. VAnderkooy, A.C. MacDonald (1998): Seasonal variations in hair zinc concentrations in Canadian and African children. - Sci. Total Environ., 84, 291-298.

GOLWER, A und F. ZEREINI (1998): Einflüsse des Straßenverkehrs auf rezente Sedimente - Langzeituntersuchungen an einem Versickerungsbecken bei Frankfurt am Main. - Geol. Jb. Hessen, 126, 47-70, Wiesbaden.

GRUND, W.; W.D. SCHNEIDER und W. WiesneR (1980): Der Mangangehalt des Haares, ein Kriterium für die Bewertung des Expositionsrisikos der Elektroschweißer. - J. Radioanal. Chem., 58, 319-326.

HARRE, W. und H.W. Walther (1957): Buntmetalle in Oberharzer Böden und Gewässern. - Zeitschrift für angew. Chemie, 69, 181.

Häussler, U., A. Nagel, M. Braun, A. ARnold (2000): External characters discriminating sibling species of European pipistrelles, Pipistrellus pipistrellus (Schreber, 1774) and P. pygmaeus (Leach, 1825). - Myotis, 37, 27-40, Bonn.

HeCkenRoth, H.; B. Pott (1988): Beiträge zum Fledermausschutz in Niedersachsen. - Naturschutz Landschaftpflege Niedersachs., 17, 1-78, Hannover. 
Helmers, E., N. Mergel (1997): Platin in belasteten Gräsern. Anstieg der Emissionen aus PKW-Abgaskatalysatoren Erster Trend aus direkten Umweltmessungen (1992-1995). - Z. Umweltchem. Ökotox., 9, (3), 147-148.

HoRN, H.H. v. (1974): Die Oker- oder Steinfeldkrankheit - Eine Umweltverschmutzung aus vergangenen Jahrhunderten und ihre Spätfolgen. - 144 S., Diss. Göttingen.

IAEA (1978): Activation of hair as an indicator of contamination of man by environmental trace element pollutants. - Rep. IAEA/RL/41, January 1977, updated IAEA/RL/50, October 1978.

Kalberlah, F. (1999): Chrom-VI-Verbindungen. - In Eikmann, T., U. Heinrich, B. HEINZOW und R. KONIETZKA (Hrsg.): Gefährdungsabschätzung von Umweltschadstoffen: Ergänzbares Handbuch toxikologischer Basisdaten und ihre Bewertung. E. Schmidt, Berlin.

Kalden, M., A. NeOcleous, K.S. Saternus und H. KIJeWSKI (1997): Die spurenkundliche Bedeutung der Molybdän- und Platingehalte von menschlichen Kopfhaaren. - In: KIJEWSKI, H. (Hrsg.): Das Haar als Spur - Spuren in Haaren, 7591, Schmidt-Römhild, Lübeck.

KATZ, S. A., A. CHATT (1988): Hair Analysis - Applications in the Biomedical and Environmental Science. - $134 \mathrm{~S}$., VCH, Weinheim.

KIJEWSKI, H. (1993): Die forensische Bedeutung der Mineralstoffgehalte in menschlichen Haaren. - 184 S., Verlag Schmidt-Römhild, Lübeck.

KIJEWSKI, H. (Hrsg.) (1997): Das Haar als Spur - Spuren in Haaren. - 152 S., Schmidt-Römhild, Lübeck.

KIJEWSKI, H. und LANGE, J. (1977): Die Beurteilung des Spurenelementgehaltes von Haaren in Kriminalistik, Toxikologie und Umweltschutz. Untersuchungen zur Wanderungskinetik von Metallionen in Keratin. - Z. Rechtsmedizin, 80, 6774, Springer, Leipzig.

KIJEWSKI, H.; H.D. LOWITZ (1982): Der Nachweis von Blei als Hydrid in Knochenbiopsieproben von Patienten mit lange zurückliegenden Bleivergiftungen. - Arch. Toxicol., 50, 301-311, Springer.

KLEIMINGER, J. (1983): Untersuchungen zur Eignung von freilebenden Wildarten als Bioindikatoren zur Erfassung von flächenhaften Schwermetallkontaminationen in Niedersachsen. - 162 S., 36 Tab., Diss. med.vet., Hannover.

KNOLLE, F. und Fh. KNOLLE (1983): Vogel- und Säugetierverluste durch Umweltschäden im Gebiet des Harzes. - Vogelkundl. Ber. Nieders., 15, (2), 47-49.

Kollmeier, H., J. Seemann, P. Wittig, Ch. Witting, G. Rothe (1985): Metallanreicherungen in Humangeweben - Chrom, Nickel, Blei, Cadmium und Zink in Lunge, Leber, Milz und Niere. - Schriftenreihe der Bundesanstalt für Arbeitsschutz, 347/VI, 150 S., Dortmund.

Koopmann, K.F. (1994): Chiroptera: Systematics. - In: J. Niethammer, H. SCHLIEMANN, D. STORCK (Eds.): Handbook of Zoology, Vol. 8, Part 60, Mammalia, 217 S., de Gryter, Berlin, New York.

KRAuSE, C. und M. CHUTSCH (Hrsg.) (1987): Haaranalyse in Medizin und Umwelt. Schriftenreihe des Vereins Wasser-, Boden- und Lufthygiene, 71, Thieme, Stuttgart. 
Krause, C., C. Schulz, K. Becker, W. Bernigau, K. Hoffmann et al. (1997): Umwelt-Survey 1990/92 - Band Ib: Deskription der Spurenelementgehalte im Haar der Bevölkerung der Bevölkerung in der Bundesrepublik Deutschland. WaBoLu-Hefte 2/96, 268 S., Berlin.

LACKI, M.J. (1994): Metal Concentrations in Guano from a Gray Bat Summer Roost. Trans. Ky. Acad. Sci., 55 (3-4), 124-126.

LEHNE, P.H. und H.-J. WeINBERG (1968): Blei und Silber; ihre letzte Gewinnung auf dem Oberharz 1967; Hüttenprozesse in Oberharzer Metallhütten. - Piper, Clausthal-Zellerfeld.

LEIßE, B. (1992): Holzschutzmittel im Einsatz - Bestandteile, Anwendungen, Umweltbelastungen. - 223 S., Bauverlag, Wiesbaden, Berlin.

LEITNER, J. und W. VOGEL (1993): Fledermäuse als Bioindikatoren - Untersuchungen aus dem Mittleren und Südlichen Burgenland. - UBA Reports 93-072, 92 S., Wien.

LILIENTHAL, H. (1992): Die Wirkung subtoxischer Bleibelastungen auf akustisch evozierte Hirnstammpotentiale: Untersuchungen an einem Primatenmodell. $115+12$ S., Diss. Univ. Düsseldorf, Verlag f. Wiss. Forschung und Technik, Wermelskirchen,.

LINDORFER, H. (1997): Relikte ehemaliger Metallgewinnung als Quelle von Schwermetall-Belastungen in rezenten Überflutungssedimenten des niedersächsischen Harzvorlandes. - 78 S., 18 Abb., 10 Tab., Anh., Dipl.arbeit Univ. Göttingen.

LitTERST, C.L., A.F. LeRoY und A.M. GuARINO (1979): Disposition and Distribution of Platinium Following Parenteral Administration of cis-Diclordiammineplatinum(II) to Animals. - Cancer Treat. Rev., 63, 1485.

LUTZ, W. (1985): Ergebnisse der Untersuchung von Rehen (Capreolus capreolus L.) und Hasen (Lepus europaeus Pallas) auf Schwermetalle und chlorierte Kohlenwasserstoffe in Nordrhein-Westfalen. - Z. Jagdwiss., 31, 153-175.

LUTZ, W.; J. SLAMECKA (1997): Vergleichende Blei- und Cadmiumbelastung des Feldhasen (Lepus europaeus PALLAS) in landwirtschaftlich und industriell genutzten Gebieten in Deutschland und der Slowakei. - Z. Jagdwiss., 43, 176-185.

MEYER, C.F.G. (1822): Beiträge zur Chorographischen Kenntnis des Flußgebietes der Innerste in den Fürstentümern Grubenhagen und Hildesheim mit besonderer Rücksicht auf die Veränderungen, die durch diesen Strom in der Beschaffenheit des Bodens und der Vegetation bewirkt worden. - Erste Anlage zur Flora des Königsreiches Hannover, Göttingen.

MiURA, T., T. KOYAMA, I. NAKAMURA (1978): Mercury content in museum and recent specimens of Chiroptera in Japan. - Bull. Environm. Contam. Toxicol., 20, (5), 696-701.

NAGEL, A. (1989): Untersuchungen zur Belastung einer Wochenstube von Zwergfledermäusen (Pipistrellus pipistrellus) mit Chlorkohlenwasserstoffen und die Belastung mehrerer Fledermausarten mit Schwermetallen. - Flattermann, 78.

NAGEL, A. (1998): Die Belastung einheimischer Fledermäuse mit Chlorkohlenwasserstoffen. - Beiträge der Akademie, 26, 95-130. 
NAGEL, A.; J. DISSER (1987): Untersuchungen zur Belastung einer Wochenstube von Zwergfledermäusen (Pipistrellus pipistrellus) mit Chlorkohlenwasserstoffen. Verh. Dt. Zool. Ges., 80, 296-297.

NAGEL, A.; J. DISSER (1990): Rückstände von Chlorkohlenwasserstoff-Pestiziden in einer Wochenstube der Zwergfledermaus (Pipistrellus pipistrellus). - Z. Säugetierk., 55, 217-225.

NAGEL, A.; S. WinteR, B. StReIT (1991): Die Belastung niedersächsischer Fledermäuse mit Chlorkohlenwasserstoffen. - Naturschutz und Landschaftpflege in Niedersachsen, 26,143-150, Hannover.

Needleman, H.L. (2000): The Removal of Lead from Gasoline: Historical and Personal Reflections. - Environmental Research, A 84, 20-35.

NeuweIleR, G. (1993): Biologie der Fledermäuse. - 350 S., 19 Tab., Thieme, Stuttgart.

Niedersä̈hsisches Landesamt für Bodenforschung (2000): Digitaler Atlas Hintergrundwerte - Natürliche chemische Gehalte von Bachsedimenten, Böden, Gesteinen und Grundwasser in Niedersachsen und Bremen. - CD-ROM, Hannover.

NoWAK, E., J. BLAB und R. BLESS (1994): Rote Liste der gefährdeten Wirbeltiere in Deutschland. - Schriftenreihe für Landschaftspflege und Naturschutz, 42, Kilda-Verlag, Greven.

NoWAK, H.; F. PREUL (1971): Untersuchungen über Blei- und Zinkgehalte in Gewässern des Westharzes. - Beih. Geol. Jb, 105, 68 S., 4 Abb., 30 Tab., 9 Taf., Hannover.

OHM, K.-H. (1991): Organochlorbelastung in Fledermäusen. - 50 S., Diss. Univ. Kiel.

PETIT, M.G., J.S.AlTENBACH (1973): A Chronological Record of Environmental Chemicals from Analysis of Stratified Vertebrate Excretion Deposited in a Sheltered Environment. - Environmental Research, 6, 339-343.

RACKOW, W. (1991): Nachweise von Blei und Cadmium im Kot des Mausohrs (Myotis myotis Borkhausen, 1797). - Nyctalus (N.F.), 4, (2), 140-144, Berlin.

REICHSBUND DEUTSCHE JäGERSCHAFT (Hrsg.) (1939): Jahrbuch der Deutschen Jägerschaft. - 4, 1938/39, Berlin.

REIDINGER, R.F. (1972): Factors influencing Arizona bat population levels. - 172 S., Ph.D. Thesis Univ. Arizona, Tucson.

RobBins, V.R. (1988): Chemical and Physical Behavior of Human Hair. - 305 S., 62 Abb., Springer.

ROSMANITH, J., H.J. EINBRODT, T. GORDON (1976): Beziehungen zwischen Blei- und Zinkniederschlägen und den Schwermetallgehalten (Pb, Zn, Cd) im Blut, Urin und in den Haaren bei Kindern. - Zbl. Bakt. Hyg (orig. B), 161, 125-136.

SchäFER, S.G., B. ElSENhANS, W. ForTh und K. Schümann (1997): Metalle. - In: MARQUARDT, H. und S.G. SCHÄFER (Hrsg.): Lehrbuch der Toxikologie. - 504549, Spektrum, Heidelberg, Berlin.

SCHÄFFLER, M. (1989): Fledermausschutz in der Region Ostalb. - Materialhefte zur Karst- und Höhlenkunde (MKH), 8, 1-48. 4 Tab., 1 Taf., Heidenheim.

SCHNEIDER, J. (1999): Schwermetalle in Böden Niedersachsens. - Arbeitshefte Boden des NLfB, 2/1999, 24 S., 3 Abb., 4 Tab., 3 Kt., Hannover. 
Schober, W.; E. GRImmberger (1998): Die Fledermäuse Europas. - 265 S., Kosmos, Stuttgart.

SchroedeR J. v.; C. Reuss (1883): Die Beschädigung der Vegetation durch Rauch und die Oberharzer Hüttenrauchschäden. - 333 S., Parey, Berlin.

Schroeder, H.A., M.D. Douglas, V. Frost, J. BALASSA (1970): Essential trace metals in man: Selenium. - J. Chron. Dis., 23, 227-243.

Schulte, A. und W. E.H. BLUM (1997): Schwermetalle in Waldökosystemen. - In: Matschullat, J., H.J. TOBSChall, H.-J. Volgt (Hrsg.): Geochemie und Umwelt, 53-74, Springer, Berlin, Heidelberg.

ScHUTKOWSKI, H. (1998): Biokulturelle Anpassungen in menschlichen Bevölkerungen - Beiträge zu einer Humanökologie. - 426 S., Habilitationsschrift Univ. Göttingen.

Schwartz, B.S., W.F. Stewart, K.I. Bolla, D. Simon, K. Bandeen-Roche, B. GoRDON, J.M. LINKS, A.C. TODD (2000): Past adult lead exposure in associated with longitudinal decline in cognitive function. - Neurology, 55, 11441150.

SEgERS-GLOCKE, Chr. (2000): Auf den Spuren einer frühen Industrielandschaft. - Arbeitshefte für Denkmalpflege in Niedersachsen, 21, 182 S., Hameln.

Skerratt, L.F.; R. Speare, L. BergeR, H. Winsor (1998): Lyssaviral infection and lead poisoning in black flying foxes from Queensland. - J. Wildl. Dis., 34, (2), 355-361.

SPÄTH, M. und M. SONNEBORN (1987): Schwermetallgehalte in menschlichen Haaren in Abhängigkeit von einer industriellen Bleibelastung. - Schriftenr. Ver. Wasser-, Boden- Lufthyg., 71, 83-89.

SPERLING, K.-R., B. BAHR (1979): Determination of Heavy Metals in Sea Water and in Marine Organisms by Flameless Atomic Absorption Spectrophotometry. - Z. Lebensm. Unters. Forsch., 168, 193-194.

SperLing, K.-R., B. BAHR, K. Kremling (1977): Heavy Metal Determination in Sea Water and in Marine Organisms with the Aid of Flameless AAS. - Z. Lebensm. Unters. Forsch., 163, 87-91.

StefFEN, D. (1994): Die Belastung der niedersächsischen Fließgewässer mit Schwermetallen und organischen Problemstoffen - Sedimentuntersuchungen im Zeitraum von 1986-1992. - Schriften des Nieders. Landesamtes für Ökologie, $107 \mathrm{~S}$., Hildesheim.

STERNER, W. (1972): Die Haaranalyse - eine besonders für epidemiologische Untersuchungen geeignete Methode zur Feststellung von Schwermetallbelastungen bei Mensch und Tier. - Arch. Lebensmittelhyg., 23, 209-213.

Streit, B. und A. NAGEL (1993a): Element assessment in tissue samples from European bats (Microchiroptera). - Fres. Envir. Bull., 2, 162-167.

Streit, B. und A. NAGEL (1993b): Heavy metal transfer by lactation in a bat colony. Fres. Envir. Bull., 2, 166-173.

Sutton, R.H. (1983): Lead Poisoning in Grey-headed Fruit Bats (Pteropus poliocephalus). - J. Wildl. Dis.,19, (3), 294-296.

Sutton, R.H. und B. HARIONO (1986): Lead Poisoning in Flying-foxes (Chiroptera:Pteropodidae). - Australian Mammology, 10, (2), 125-126. 
Sutton, R.H., P.D. WILSON (1983): Lead Poisoning in Grey-headed Fruit Bats (Pteropus poliocephalus). - J. Wildlife Dis., 19, (3), 294-296.

SWIFT, S.M., P.A. RACEY und M.I. Avery (1985): Feeding ecology of Pipistrellus pipistrellus (Chiroptera, Vesp.) during pregnancy and lactation. II. Diet. - J. Anim. Ecol., 54, 217-225.

Thies, M.; D. GREGory (1994): Residues of Lead, Cadmium, and Arsenic in Livers of Mexican Free-Tailed Bats. - Bull. Environm. Contam. Toxicol., 52, 641-648.

Uloth, W., C. Treß, R. KÖRNER, D. MAJOHR (1987): Das Verhalten von Cadmium und Blei im Fledermausguano. Mengen und Spurenelemente. - Arbeitstagung Leipzig, 107-109.

UMWELtBundesAmt (1991): Daten zur Umwelt. - 675 S., E. Schmidt, Berlin.

UMWELTBUNDESAMT (1997): Daten zur Umwelt. - 570 S., E. Schmidt, Berlin.

UMWELTBUNDESAMT (2000): Schadstoffminimierung durch verbesserte Kraftstoffe. Umweltdaten Deutschland 1998 (Internetausgabe), Berlin.

Wasserchemische Gesellschaft und Normenausschub WasserWesen (NAW) im DIN (2000): Deutsche Einheitsverfahren zur Wasser-, Abwasser- und Schlamm-Untersuchung. - Wiley-VCH und Beuth, Weinheim, Berlin.

Wietlisbach, V., M. Rickenbach, M. Berode, M. Guillemin (1995): Time Trend and Determinants of Blood Lead Levels in a Swiss Population over a Transition Period (1984-1993) from Leaded to Unleaded Gasoline Use. - Environmental Research, 68, 82-90.

WIRTH, C. W. und C. Gloxhuber (1994): Toxikologie. - 512 S., Thieme, Stuttgart.

Woloszyn, B.W., K. KozAKIEWICZ, K. PielichoWsKI et al. (1999): Physiochemical Analysis of Bat Hair by Atomic Absorption Spectroscopy and Thermal Techniques. - Bat research news, 40, (3), 148-149.

Zereini, F., F. Alt (1999): Emissionen von Platinmetallen. - 327 S., 74 Abb., 62 Tab., Springer, Berlin.

ZereinI, F., F. Alt (Eds.) (2000): Anthropogenic Platinium-Group Element Emissions - Their Impact of Man and Environment. - 308 S., 49 Abb., 80 Tab., Spinger.

Zereini, F., F. Alt, K. Rankenbrug, J-M. Beyer, S. Artelt (1997a): Verteilung von Platingruppenelementen (PGE) in den Umweltkompartimenten Boden, Schlamm, Straßenstaub, Straßenkehrgut und Wasser. - Z. Umweltchem. Ökotox., 9, (4), 193-200, Landsberg.

Zereini, F., F. DiRKSEN, B. SKeRstrupp, H. URBAN (1998): Sources of Anthopogenic Platinium-Group Elements (PGE): Automotive Catalysts versus PGEProcessing Industries. - Environ. Science and Pollution Reseach, 5, (4), 223230, Landsberg.

Zereini, F., B. Skerstrupp, F. Alt, E. Helmers, H. Urban (1997b): Geochemical behavior of platinium-group elements (PGE) in particulate emissions by automobile exhaust catalysts: experimental results and environmental investigations. - Sci. Total Environment, 206, 137-146.

ZereinI, F., C. ZIENTEK, H. URBAN (1993): Konzentration und Verteilung von Platingruppenelementen (PGE) in Böden. - Z. Umweltchem. Ökotox., 5, (3), 130134, Landsberg.

ZOOK, B.C., R.M. SAUER und F.M. GARNER (1970): Lead poisoning in Australian fruit bats. (Pteropus poliocephalus). - J. Am. Vet. Med. Assoc., 157, (5), 691-694. 
ZüLL, M. (1995): Altstandort "Erzbergwerk Grund" (Harz): Analyse von Wässern und Sedimenten mit photometrischen und atomspektrometrischen Methoden. Zertifikatsabschlußarbeit FH Hildesheim/Holzminden, FB Forstwirtschaft, Ergänzungsstudium "Umwelt- und Naturschutz", $58 \mathrm{~S}$.

ZYRNICKI, W., J. PIELICHOWSKI, B.W. WOLOSZYN (1996): Preliminary Results of Studies Concerning the Contents of Metals in Bat Hair. - Bat research news, 37, 103. 


\section{ANHANG}

Zusammenstellung der Meßdaten 
Anhang 1:

Biometrische Daten von untersuchten Fledermäusen aus dem Formenkreis Zwergfledermaus (P. pipistrellus und $P$. pygmaeus)

\begin{tabular}{|c|c|c|c|c|c|c|c|c|c|c|c|c|}
\hline Tier-Nr. & Fundort & $\begin{array}{c}\text { TK25 } \\
\text { Blatt-Nr. }\end{array}$ & Quadr. & Funddatum & Geschl. & Alter & $\begin{array}{l}\text { Unterarmlänge } \\
\text { [mm] }\end{array}$ & $\begin{array}{c}\text { Länge Finger- } \\
\text { gliedes I, Finger } 3 \\
\text { [mm] }\end{array}$ & $\begin{array}{c}\text { Länge Finger- } \\
\text { gliedes II, Finger } 3 \\
{[\mathrm{~mm}]}\end{array}$ & $\begin{array}{c}\text { Länge Finger- } \\
\text { gliedes III, Finger } 3 \\
\text { [mm] }\end{array}$ & $\begin{array}{l}\text { FGII-FGIII } \\
\text { [mm] }\end{array}$ & $\begin{array}{l}\text { Quotient } \\
\text { FGII/FGIII }\end{array}$ \\
\hline 87003 & Winzenburg, LK Hildesheim & 4025 & 4 & 17.8.1987 & w & adult & 32,0 & 10,9 & 8,3 & 7,7 & 0,6 & 1,08 \\
\hline 87005 & Braunschweig, Jasperallee & 3729 & 1 & 3.7.1987 & $\mathrm{m}$ & adult & 30,0 & 10,1 & 7,8 & 6,4 & 1,4 & 1,23 \\
\hline 89004 & Stadt Osterode a.H. & 4227 & 4 & 1.12 .1989 & $\mathrm{~m}$ & adult & 32,0 & 10,1 & 8,2 & 6,2 & 1,9 & 1,31 \\
\hline 89009 & Stadt Osterode a.H. & 4227 & 4 & 1989 & $\mathrm{~m}$ & subad. & 30,9 & 10,1 & 7,5 & 6,4 & 1,1 & 1,18 \\
\hline 90001 & Hedemünden, LK Göttingen & 4624 & 2 & 5.6 .1990 & $\mathrm{~m}$ & adult & 31,9 & 11,4 & 9,1 & 8,3 & 0,8 & 1,10 \\
\hline 90009 & Osterode a.H. - OT Förste & 4227 & 3 & 13.5.1990 & w & adult & 32,5 & 11,5 & 8,3 & 7,0 & 1,3 & 1,18 \\
\hline 90010 & Eisdorf, LK Osterode a.H. & 4227 & 3 & 9.7 .1990 & w & juv. & 26,5 & 6,6 & 5,8 & 5,1 & 0,6 & 1,13 \\
\hline 90013 & Lindau, LK Northeim & 4326 & 2 & 25.6.1990 & $\mathrm{m}$ & juv. & 31,0 & 8,5 & 6,4 & 6,1 & 0,3 & 1,05 \\
\hline 91021 & Nienstedt, LK Osterode & 4227 & 3 & 15.7.1991 & $\mathrm{m}$ & juv. & 22,8 & 6,9 & 4,8 & 4,0 & 0,8 & 1,20 \\
\hline 91022 & Osterode a.H. - OT Förste & 4227 & 3 & 22.7.1991 & w & subad. & 28,0 & 8,6 & 5,9 & 5,8 & 0,2 & 1,03 \\
\hline 92001 & Amelinghausen, LK Lüneburg & 2827 & 3 & 31.8.1992 & $\mathrm{m}$ & adult & 30,8 & 10,1 & 8,0 & 6,9 & 1,1 & 1,16 \\
\hline 93001 & Wunstorf, LK Hannover & 3522 & 4 & 3.12 .1993 & w & adult & 30,7 & 10,6 & 8,3 & 7,7 & 0,6 & 1,08 \\
\hline 94004 & Badenhausen, LK Osterode a.H. & 4227 & 1 & 14.2 .94 & $\mathrm{~m}$ & adult & 31,5 & 10,7 & 8,5 & 7,5 & 1,0 & 1,13 \\
\hline 94011 & Eisdorf, LK Osterode a.H. & 4227 & 3 & 23.7.94 & $\mathrm{m}$ & subad. & 30,8 & 9,6 & 7,7 & 6,9 & 0,8 & 1,12 \\
\hline 94015 & Osterode a.H. - OT Förste & 4227 & 3 & 24.4.94 & w & adult & 31,4 & 9,6 & 8,0 & 7,8 & 0,2 & 1,02 \\
\hline 94016 & Bad Lauterberg i.H. & 4328 & 4 & 7.6 .94 & w & adult & 32,0 & 9,6 & 8,0 & 6,7 & 1,3 & 1,19 \\
\hline 94034 & Elbingerode, LK Osterode a.H. & 4327 & 2 & 21.4 .94 & $\mathrm{~m}$ & subad. & 29,5 & 9,6 & 7,5 & 7,5 & 0,0 & 1,00 \\
\hline 94039 & Varmissen, LK Göttingen & 4424 & 4 & 21.7 .94 & $\mathrm{~m}$ & adult & 31,4 & 10,9 & 8,0 & 7,0 & 1,0 & 1,14 \\
\hline 95009 & Hachenhausen, LK Northeim & 4126 & 1 & 5.7 .95 & $\mathrm{~m}$ & adult & 31,6 & 9,6 & 7,5 & 6,6 & 1,0 & 1,15 \\
\hline 95010 & Osterode a.H. - OT Schwiegershsn. & 4327 & 1 & 16.7.95 & w & subad. & 31,0 & 8,0 & 6,9 & 5,1 & 1,8 & 1,34 \\
\hline 95012 & Stadt Osterode a.H. & 4227 & 4 & 6.9 .95 & w & adult & 29,5 & 10,4 & 7,7 & 7,5 & 0,2 & 1,02 \\
\hline
\end{tabular}




\begin{tabular}{|c|c|c|c|c|c|c|c|c|c|c|c|c|}
\hline Tier-Nr. & Fundort & $\begin{array}{c}\text { TK25 } \\
\text { Blatt-Nr. }\end{array}$ & Quadr. & Funddatum & Geschl. & Alter & $\begin{array}{l}\text { Unterarmlänge } \\
{[\mathrm{mm}]}\end{array}$ & $\begin{array}{c}\text { Länge Finger- } \\
\text { gliedes I, Finger } 3 \\
\text { [mm] }\end{array}$ & $\begin{array}{c}\text { Länge Finger- } \\
\text { gliedes II, Finger } 3 \\
\text { [mm] }\end{array}$ & $\begin{array}{c}\text { Länge Finger- } \\
\text { gliedes III, Finger } 3 \\
\text { [mm] }\end{array}$ & $\begin{array}{c}\text { FGIII-FGIII } \\
{[\mathrm{mm}]}\end{array}$ & $\begin{array}{l}\text { Quotient } \\
\text { FGII/FGIII }\end{array}$ \\
\hline 95014 & Ührde, LK Osterode a.H. & 4227 & 3 & 25.6 .95 & $\mathrm{~m}$ & adult & 29,7 & 10,4 & 8,8 & 6,9 & 1,9 & 1,28 \\
\hline 95016 & Hammenstedt, LK Northeim & 4326 & 1 & 8.7 .95 & w & adult & 37,7 & 10,1 & 8,8 & 7,2 & 1,6 & 1,22 \\
\hline 95020 & Hannover-Schulenburg & 3524 & 4 & 23.8.95 & $\mathrm{m}$ & adult & 30,3 & 9,9 & 8,3 & 7,2 & 1,1 & 1,16 \\
\hline 95021 & Osterode a.H. - OT Förste & 4227 & 3 & 19.10 .95 & $\mathrm{~m}$ & adult & 31,7 & 10,7 & 8,8 & 7,2 & 1,6 & 1,22 \\
\hline 96001 & Hannover-Kleefeld & 3624 & 2 & 11.8 .96 & $\mathrm{~m}$ & adult & 31,0 & 10,4 & 8,6 & 6,4 & 2,2 & 1,35 \\
\hline 96002 & Hannover, Rühmkorfstr. & 3524 & 3 & 22.8.1996 & $\mathrm{m}$ & adult & 30,9 & 10,1 & 8,0 & 7,2 & 0,8 & 1,11 \\
\hline 96003 & Hannover, Rühmkorfstraße & 3524 & 3 & 22.8.1996 & $\mathrm{m}$ & adult & 30,7 & 11,7 & 9,4 & 6,6 & 2,9 & 1,44 \\
\hline 96012 & Eisdorf, LK Osterode a.H. & 4227 & 3 & 30.7 .96 & $\mathrm{~m}$ & subad. & 27,0 & 7,2 & 4,8 & 4,8 & 0,0 & 1,00 \\
\hline 96014 & Osterode a.H. - OT Förste & 4227 & 3 & 7.7 .96 & w & juv. & 25,5 & 6,4 & 4,2 & 5,1 & $-1,0$ & 0,81 \\
\hline 96026 & Stadt Hannover & 3624 & 1 & 24.8 .96 & $\mathrm{~m}$ & subad. & 29,8 & 10,4 & 8,6 & 6,7 & 1,9 & 1,29 \\
\hline 97001 & $\begin{array}{l}\text { Osterode-Clausthal, an der Bundes- } \\
\text { straße B } 241\end{array}$ & 4227 & 2 & 6.11 .1997 & $\mathrm{~m}$ & adult & 32,1 & 11,5 & 8,3 & 7,7 & 0,6 & 1,08 \\
\hline 97006 & Bartolfelde Landkreis Osterode & 4428 & 2 & 17.7.97 & w & subad. & 31,9 & 6,4 & 5,6 & 5,6 & 0,0 & 1,00 \\
\hline 97013 & Elvershausen Landkreis NOM & 4226 & 4 & 2.8.97 & $\mathrm{m}$ & subad. & 27,8 & 7,2 & 6,1 & 5,4 & 0,6 & 1,12 \\
\hline 97014 & Harriehausen Landkreis NOM & 4126 & 4 & 6.7 .97 & $\mathrm{~m}$ & subad. & 28,1 & 6,7 & 5,6 & 5,1 & 0,5 & 1,09 \\
\hline 98001 & Osterode am Harz - Ortsteil Freiheit & 4227 & 4 & 19.3.1998 & $\mathrm{m}$ & adult & 31,2 & 10,4 & 8,3 & 6,4 & 1,9 & 1,30 \\
\hline 98003 & Osterode a.H. - OT Schwiegershsn. & 4327 & 1 & 31.5 .1998 & w & adult & 34,8 & 10,7 & 10,7 & 6,2 & 4,5 & 1,72 \\
\hline 98004 & Osterode a.H. - OT Schwiegershsn. & 4327 & 1 & 31.5 .1998 & $\mathrm{~m}$ & adult & 34,0 & 10,7 & 9,4 & 5,6 & 3,8 & 1,69 \\
\hline 98005 & Osterode a.H. - OT Förste & 4227 & 3 & 22.7.1998 & w & juv. & 25,4 & 5,6 & 4,6 & 4,5 & 0,2 & 1,04 \\
\hline 98007 & Steina/Bad Sachsa & 4429 & 1 & 12.7.1998 & w & juv. & 29,0 & 6,4 & 5,6 & 5,3 & 0,3 & 1,06 \\
\hline 98008 & Steina/Bad Sachsa & 4427 & 1 & 12.7.1998 & w & juv. & 26,9 & 8,0 & 5,4 & 5,4 & 0,0 & 1,00 \\
\hline 98012 & Osterode a.H. - Ortsteil Freiheit & 4227 & 4 & 2.6 .1998 & $\mathrm{~m}$ & adult & 30,0 & 10,4 & 8,0 & 6,1 & 1,9 & 1,32 \\
\hline 98013 & Osterode a.H. - Ortsteil Freiheit & 4227 & 4 & 2.6.1998 & $\mathrm{m}$ & adult & 31,7 & 10,4 & 7,8 & 7,2 & 0,6 & 1,09 \\
\hline 98014 & Barbis, Bad Lauterberg i.H. & 4328 & 4 & 10.7.1998 & w & adult & 29,7 & 8,8 & 6,6 & 6,2 & 0,3 & 1,05 \\
\hline 98016 & Osterode a.H. - Ortsteil Freiheit & 4227 & 4 & 4.7.1998 & $\mathrm{m}$ & adult & 30,2 & 10,1 & 8,0 & 6,4 & 1,6 & 1,25 \\
\hline 98017 & Osterode a.H. - OT Schwiegershsn. & 4327 & 1 & 7.6 .1998 & $\mathrm{~m}$ & adult & 31,6 & 10,4 & 8,6 & 6,2 & 2,4 & 1,38 \\
\hline 98019 & Bad Sachsa - LK Osterode a.H. & 4429 & 1 & 1998 & $\mathrm{~m}$ & subad. & 27,7 & 6,7 & 4,8 & 4,8 & 0,0 & 1,00 \\
\hline
\end{tabular}




\begin{tabular}{|c|c|c|c|c|c|c|c|c|c|c|c|c|}
\hline Tier-Nr. & Fundort & $\begin{array}{c}\text { TK25 } \\
\text { Blatt-Nr. }\end{array}$ & Quadr. & Funddatum & Geschl. & Alter & $\begin{array}{c}\text { Unterarmlänge } \\
{[\mathrm{mm}]}\end{array}$ & $\begin{array}{c}\text { Länge Finger- } \\
\text { gliedes I, Finger } 3 \\
{[\mathrm{~mm}]}\end{array}$ & $\begin{array}{c}\text { Länge Finger- } \\
\text { gliedes II, Finger } 3 \\
{[\mathrm{~mm}]}\end{array}$ & $\begin{array}{c}\text { Länge Finger- } \\
\text { gliedes III, Finger } 3 \\
{[\mathrm{~mm}]}\end{array}$ & $\begin{array}{c}\text { FGII-FGIII } \\
\text { [mm] }\end{array}$ & $\begin{array}{l}\text { Quotient } \\
\text { FGII/FGIII }\end{array}$ \\
\hline 98020 & Osterode am Harz - Ortsteil Freiheit & 4227 & 4 & 26.6.1998 & $\mathrm{m}$ & subad. & 31,8 & 10,4 & 7,2 & 6,4 & 0,8 & 1,13 \\
\hline 98021 & Sieber & 4328 & 2 & 21.7.1998 & ? & juv. & 21,0 & 9,6 & 6,6 & 6,1 & 0,5 & 1,08 \\
\hline 98022 & Osterode a.H. - OT Förste & 4227 & 3 & 20.7.1998 & $\mathrm{m}$ & adult & 33,0 & 6,4 & 4,8 & 4,2 & 0,6 & 1,15 \\
\hline 98023 & Osterode am Harz - Ortsteil Freiheit & 4227 & 3 & 7.6 .1998 & $\mathrm{~m}$ & adult & 32,7 & 11,2 & 9,0 & 6,9 & 2,1 & 1,30 \\
\hline 98031 & Krebeck, Landkreis Göttingen & 4426 & 2 & 22.6.1998 & $\mathrm{m}$ & juv. & 23,2 & 4,0 & 3,7 & 3,7 & 0,0 & 1,00 \\
\hline 98056 & Stadt Osterode a.H. & 4227 & 4 & Okt. 1998 & w & adult & 33,0 & 11,4 & 9,6 & 8,3 & 1,3 & 1,15 \\
\hline 98059 & Stadt Osterode a.H. & 4227 & 4 & Okt. 1998 & w & adult & 33,0 & 11,8 & 9,4 & 7,5 & 1,9 & 1,26 \\
\hline 98060 & Stadt Osterode a.H. & 4227 & 4 & Okt. 1998 & w & adult & 30,9 & 10,4 & 8,0 & 7,4 & 0,6 & 1,09 \\
\hline 98066 & Stadt Osterode a.H. & 4227 & 4 & Okt. 1998 & $\mathrm{~m}$ & adult & 29,5 & 10,4 & 8,0 & 6,4 & 1,6 & 1,25 \\
\hline 98068 & Stadt Osterode a.H. & 4227 & 4 & Okt. 1998 & $\mathrm{~m}$ & adult & 31,4 & 10,9 & 8,8 & 6,7 & 2,1 & 1,31 \\
\hline 98070 & Stadt Osterode a.H. & 4227 & 4 & Okt. 1998 & $\mathrm{~m}$ & adult & 31,5 & 10,6 & 8,0 & 7,7 & 0,3 & 1,04 \\
\hline 98071 & Stadt Osterode a.H. & 4227 & 4 & Okt. 1998 & $\mathrm{~m}$ & adult & 30,3 & 10,1 & 8,0 & 6,6 & 1,4 & 1,22 \\
\hline 98072 & Stadt Osterode a.H. & 4227 & 4 & Okt. 1998 & $\mathrm{~m}$ & adult & 31,5 & 12,2 & 8,3 & 6,9 & 1,4 & 1,21 \\
\hline 98081 & Stadt Osterode a.H. & 4227 & 4 & 19.8.1998 & w & subad. & 31,0 & 10,6 & 8,3 & 7,4 & 1,0 & 1,13 \\
\hline 98082 & Stadt Osterode a.H. & 4227 & 4 & 19.8.1998 & $\mathrm{m}$ & subad. & 30,2 & 11,2 & 8,8 & 6,9 & 1,9 & 1,28 \\
\hline 98083 & Osterode a.H. - OT Förste & 4227 & 3 & 6.9 .1998 & w & adult & 31,4 & 10,4 & 8,0 & 6,7 & 1,3 & 1,19 \\
\hline 98085 & Stadt Osterode a.H. & 4227 & 4 & 3.9.1998 & $\mathrm{m}$ & adult & 31,7 & 12,0 & 8,8 & 6,2 & 2,6 & 1,41 \\
\hline 98086 & Stadt Osterode a.H. & 4227 & 4 & 3.9 .1998 & $\mathrm{~m}$ & adult & 29,5 & 10,4 & 8,0 & 7,2 & 0,8 & 1,11 \\
\hline 98087 & Stadt Osterode a.H. & 4227 & 4 & 8.9.1998 & w & adult & 33,0 & 10,9 & 8,3 & 6,7 & 1,6 & 1,24 \\
\hline 98088 & Salzgitter-Bruchmachtersen & 3827 & 2 & 20.12.1998 & w & adult & 32,0 & 10,7 & 8,6 & 6,9 & 1,8 & 1,26 \\
\hline 98093 & Grußendorf, LK Gifhorn & 3430 & 3 & 6.98 & $\mathrm{~m}$ & adult & 31,1 & 11,7 & 8,6 & 6,7 & 1,9 & 1,29 \\
\hline 99006 & Osterode a.H. - OT Schwiegershsn. & 4327 & 1 & 5.5.1999 & w & adult & 31,0 & 10,6 & 8,3 & 8,0 & 0,3 & 1,04 \\
\hline 99027 & Bückeburg, LK Schaumburg & 3720 & 1 & Juli 1999 & $?$ & adult & 31,2 & 11,5 & 9,4 & 7,8 & 1,6 & 1,20 \\
\hline
\end{tabular}




\section{Anhang 2:}

Meßwerte der Bestimmung des Wasseraufnahmevermögen von Fledermaus- und Humanhaar

a) Fledermaushaar:

\begin{tabular}{|c|c|c|c|c|c|}
\hline Tier-Nr. & $\begin{array}{l}17 \% \text { rel. Luft- } \\
\text { feuchte }\end{array}$ & $\begin{array}{c}35 \% \text { rel. Luft- } \\
\text { feuchte }\end{array}$ & $\begin{array}{l}50 \% \text { rel. } \\
\text { Luftfeuchte }\end{array}$ & $\begin{array}{l}81 \% \text { rel. Luft- } \\
\text { feuchte }\end{array}$ & $\begin{array}{l}98 \% \text { rel. Luft- } \\
\text { feuchte }\end{array}$ \\
\hline 87001 & $20,24(-7,33 \%)$ & $21,35(-0,49 \%)$ & 21,84 & $23,950(+9,65 \%)$ & $25,81(+18,15 \%)$ \\
\hline 87002 & $11,64(-7,18 \%)$ & $12,24(-0,26 \%)$ & 12,54 & $13,67(+9,00 \%)$ & $14,67(+16,95 \%)$ \\
\hline 92001 & $27,70(-7,85 \%)$ & $29,28(-0,71 \%)$ & 30,06 & $36,65(+9,37 \%)$ & $38,95(+16,92 \%)$ \\
\hline 96001 & $30,90(-7,40 \%)$ & $32,58(-0,75 \%)$ & 33,37 & $32,88(+9,82 \%)$ & $35,16(+16,71 \%)$ \\
\hline $98033^{*}$ & $17,21(-10,22 \%)$ & $18,55(-3,18 \%)$ & 19,17 & $21,75(+13,45 \%)$ & $23,62(+23,19 \%)$ \\
\hline Mittelwert** & $-7,44 \%$ & $-2,24 \%$ & $0 \%$ & $+9,46 \%$ & $+17,18 \%$ \\
\hline
\end{tabular}

*) Plecotus auritus - Braunes Langohr

**) arithm. Mittel der Tiere Nr. 87001, 87002,92001,96001 (=P. pipistrellus)

b) Humanhaar:

\begin{tabular}{cccccc}
\hline \hline Tier-Nr. & $\begin{array}{c}\mathbf{1 7} \% \text { rel. Luft- } \\
\text { feuchte }\end{array}$ & $\begin{array}{c}\text { 35\% rel. Luft- } \\
\text { feuchte }\end{array}$ & $\begin{array}{c}\mathbf{5 0} \% \text { rel. } \\
\text { Luftfeuchte }\end{array}$ & $\begin{array}{c}\mathbf{8 1} \% \text { rel. Luft- } \\
\text { feuchte }\end{array}$ & $\begin{array}{c}\mathbf{9 8} \% \text { rel. Luft- } \\
\text { feuchte }\end{array}$ \\
\hline Probe 1 & $66,22(-7,62 \%)$ & $69,41(-2,00 \%)$ & 71,68 & $78,49(+9,50 \%)$ & $84,15(+17,39 \%)$ \\
Probe 2 & $49,17(-7,59 \%)$ & $51,38(-1,82 \%)$ & 53,21 & $57,99(+8,98 \%)$ & $62,83(+18,07 \%)$ \\
Probe 3 & $75,08(-7,46 \%)$ & $77,90(-2,41 \%)$ & 81,13 & $88,53(+9,12 \%)$ & $95,18(+17,31 \%)$ \\
Probe 4 & $61,12(-8,45 \%)$ & $63,66(-2,51 \%)$ & 66,76 & $72,29(+8,28 \%)$ & $78,14(+17,04 \%)$ \\
Probe 5 & $63,73(-7,52 \%)$ & $65,93(-2,77 \%)$ & 68,91 & $75,15(+9,05 \%)$ & $81,00(+17,49 \%)$ \\
\hline Mittelwert & $-7,73 \%$ & $-2,30 \%$ & $0 \%$ & $+8,99 \%$ & $+17,46 \%$ \\
\hline \hline
\end{tabular}

c) Leergefäße (Blindwert):

\begin{tabular}{|c|c|c|c|c|c|}
\hline Tier-Nr. & $\begin{array}{l}17 \% \text { rel. Luft- } \\
\text { feuchte }\end{array}$ & $\begin{array}{l}35 \% \text { rel. Luft- } \\
\text { feuchte }\end{array}$ & $\begin{array}{c}50 \% \text { rel. } \\
\text { Luftfeuchte }\end{array}$ & $\begin{array}{l}81 \% \text { rel. Luft- } \\
\text { feuchte }\end{array}$ & $\begin{array}{l}98 \% \text { rel. Luft- } \\
\text { feuchte }\end{array}$ \\
\hline Probe 1 & $0,99716(+0,003 \%)$ & $0,99711(-0,002 \%)$ & 0,99713 & $0,99749(+0,036 \%)$ & $0,99753(+0,040 \%)$ \\
\hline Probe 2 & $1,01247(-0,006 \%)$ & $1,01259(+0,006 \%)$ & 1,01253 & $1,01288(+0,035 \%)$ & $1,01294(+0,040 \%)$ \\
\hline Probe 3 & $1,00934(-0,002 \%)$ & $1,00935(-0,001 \%)$ & 1,00936 & $1,00974(+0,038 \%)$ & $1,01023(+0,086 \%)$ \\
\hline Probe 4 & $0,99715(+0,004 \%)$ & $0,99708(-0,003 \%)$ & 0,99711 & $0,99738(+0,027 \%)$ & $0,99751(+0,040 \%)$ \\
\hline Probe 5 & $0,97954(0,000 \%)$ & $0,97959(-0,005 \%)$ & 0,97954 & $0,97994(+0,041 \%)$ & $0,97999(+0,046 \%)$ \\
\hline Mittelwert & $<0,001 \%$ & $-0,001 \%$ & $0 \%$ & $+0,035 \%$ & $+0,050 \%$ \\
\hline
\end{tabular}




\section{Anhang 3}

Verfahrenskenndaten der Schwermetallanalytik 
Ermittlung der Kalibrierfunktion

und der Verfahrenskenndaten nach DIN 38 402 - A 51

Element/Verbindung Analysenverfahren:

Blei

DIN 38406-E6-3

Datum der Kalibrierung: $\quad 28.01 .1999$

Kenndaten der linearen Regression:

Steigung:

y-Achsabschnit:

Reststandardabweichung:

Verfahrensstandardabweichung

Verfahrensvariationskoeffizient:

Prüfgrößen:

$a=\quad-1,067 E+00$

$S_{y}=\quad 2,377 E+\infty$

$S_{x 0}=\quad 1,672 \mathrm{E}-01$

$V_{x 0}=3,04 \%$

$Y_{p}=\quad 4,286 E+\infty 00$

$x_{p}=\quad 7,406 \mathrm{E}-01$

$x_{p} / x_{1}=\quad 74,1 \%$

entspr. rechnerischer Bestimmungsgrenze

Pearsonscher Korrelationskoeffizient

$9,986 \mathrm{E}-01$

Graphische Darstellung der linearen Kalibrierfunktion:

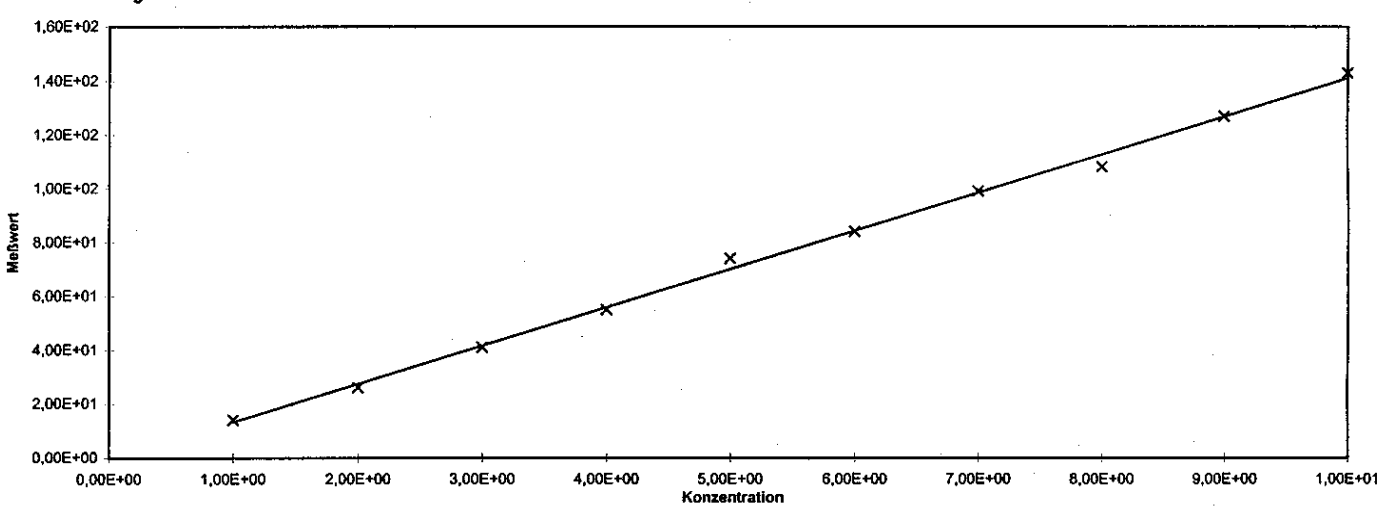

CD-G0800.XLS

Ermittlung der Kalibrierfunktion und der Verfahrenskenndaten

\begin{tabular}{|c|c|c|}
\hline $\begin{array}{l}\text { Element/Verbindung: } \\
\text { Analysenverfahren: }\end{array}$ & $\begin{array}{l}\text { Cadmium } \\
\text { GF-AAS }\end{array}$ & Datum $\operatorname{der} M$ \\
\hline \multicolumn{3}{|l|}{ Kenndaten der linearen Regression: } \\
\hline Steigung: & $b=$ & $7,591 \mathrm{E}-02$ \\
\hline y-Achsabschnitt: & $a=$ & $4,200 \mathrm{E}-03$ \\
\hline Reststandardabweichung: & $s_{y}=$ & $2,368 \mathrm{E}-03$ \\
\hline Verfahrensstandardabweichung: & $s_{x 0}=$ & $3,120 \mathrm{E}-02$ \\
\hline Verfahrensvariationskoeffizient: & $v_{x 0}=$ & $2,84 \%$ \\
\hline \multirow[t]{3}{*}{ Prüfgrößen: } & $Y_{p}=$ & $9,534 E-03$ \\
\hline & $x_{p}=$ & $1,383 E-01$ \\
\hline & $x_{p} / x_{1}=$ & $69,2 \%$ \\
\hline Pearsonscher Korrelationskoeffiz & zient: & 9,988E-01 \\
\hline
\end{tabular}

Graphische Darstellung der linearen Kalibrierfunktion:

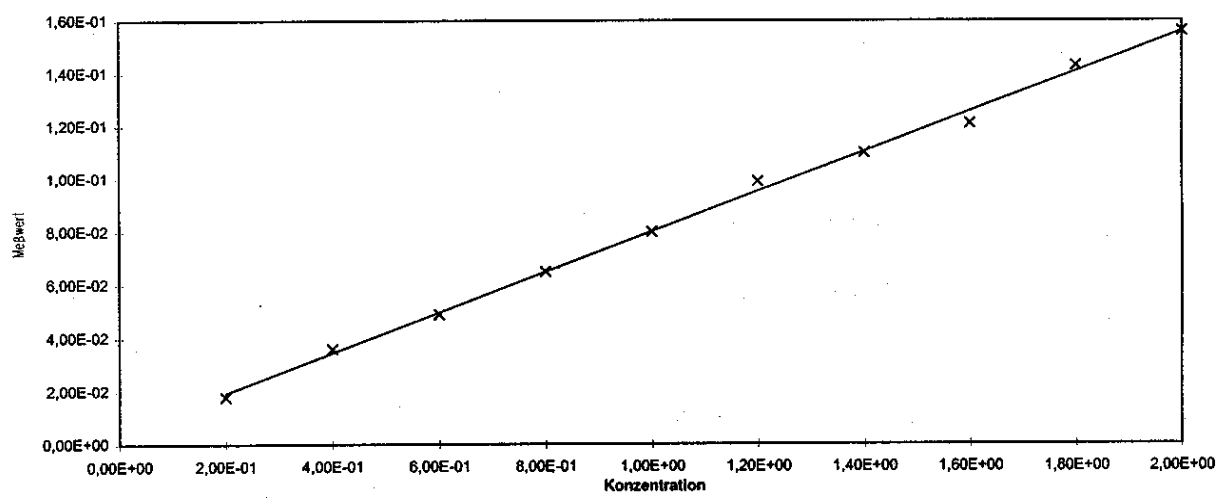


Kenndaten der linearen Regression:

$$
\text { Steigung: }
$$

$y$-Achsabschnit:

Reststandardabweichung:

Verfahrensstandardabweichung:

Verfahrensvariationskoeffizient:

Prüfgrößen:

Pearsonscher Korrelationskoeffizien:

Graphische Darstellung der linearen Kalibrierfunktion:

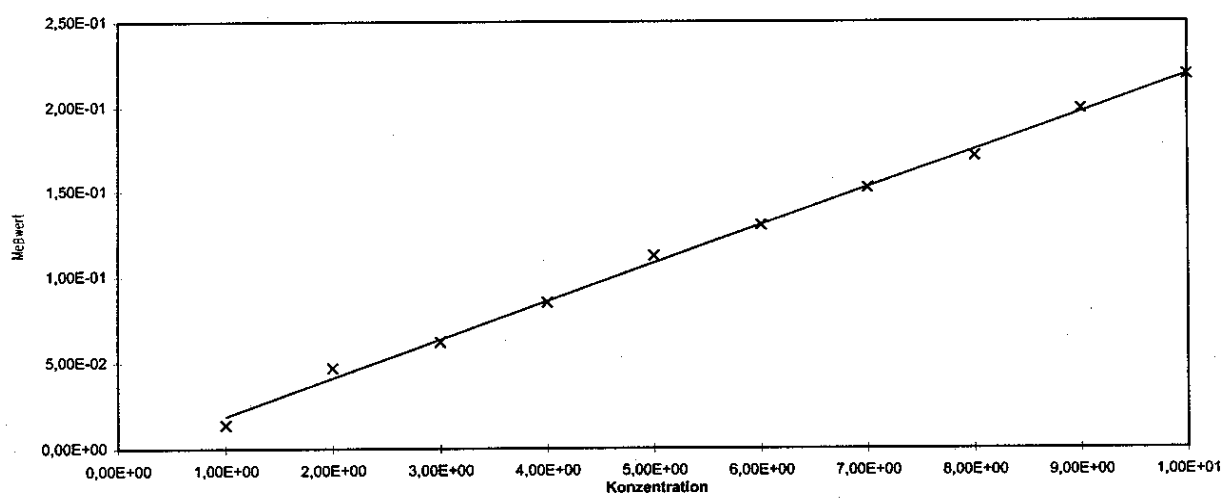

KAL_CUM.XLS Ermittlung der Kalibrierfunktion und der Verfahrenskenndaten

Element/Nerbindung: Kupfer $\quad$ Datum der Messung: 27.11 .1999 Analysenverfahren:

Kenndaten der linearen Regression:

Steigung:

y-Achsabschnitt:

Reststandardabweichung:

Verfahrensstandardabweichung:

Verfahrensvariationskoeffizient:

Prütgrößen:

$b=\quad 9,368 E+01$

$\mathrm{a}=\quad$ \#\#\#\#\#\#\#\#

$S_{\mathrm{y}}=\quad 6,909 \mathrm{E}+00$

$S_{x 0}=\quad 7,375 E-02$

$V_{x 0}=\quad 7,93 \%$

$Y_{D}=\quad 8,281 E+00$

$x_{p}=3,000 E-01$

$X_{p} / x_{1}=\quad 309,0 \%$

Pearsonscher Korrelationskoeffizient:

hische Darstellung der linearen Kalibrierfunktion

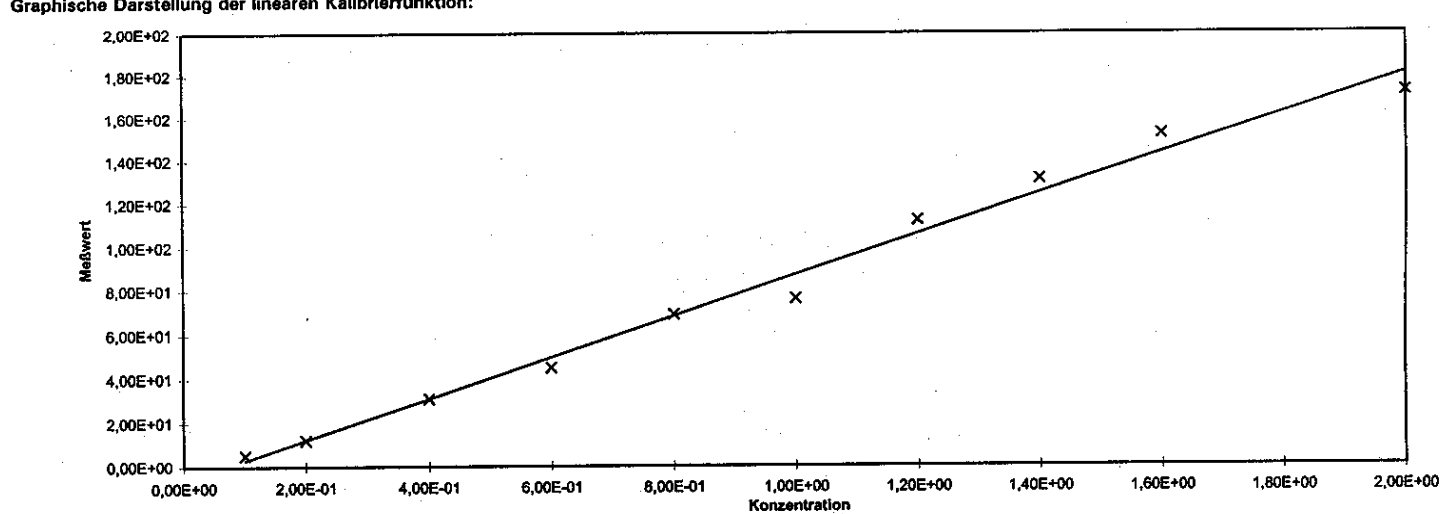


Ermittlung der Kalibrierfunktion

und der Verfahrenskenndaten

ElementVerbindung

Nicke

Datum der Messung: $\quad 22.05 .00$

Kenndaten der linearen Regression

Steigung:

y-Achsabschnitt:

Reststandardabweichung:

Verfahrensstandardabweichung:

Verfahrensvariationskoeffizient:

Prüfgrößen:

Pearsonscher Korrelationskoeffizient:

$\begin{array}{lr}b= & 1,743 E-02 \\ a== & -7,000 E-03 \\ S_{y}= & 5,452 E-03 \\ S_{x 0}= & 3,128 E-01 \\ V_{x 0}= & 2,91 \% \\ Y_{p}= & 5,279 E-03 \\ X_{p}= & 1,387 E+00 \\ X_{p} / X_{1}= & 70,9 \% \\ r= & 9,988 E-01\end{array}$

Graphische Darstellung der linearen Kalibrierfunktion:

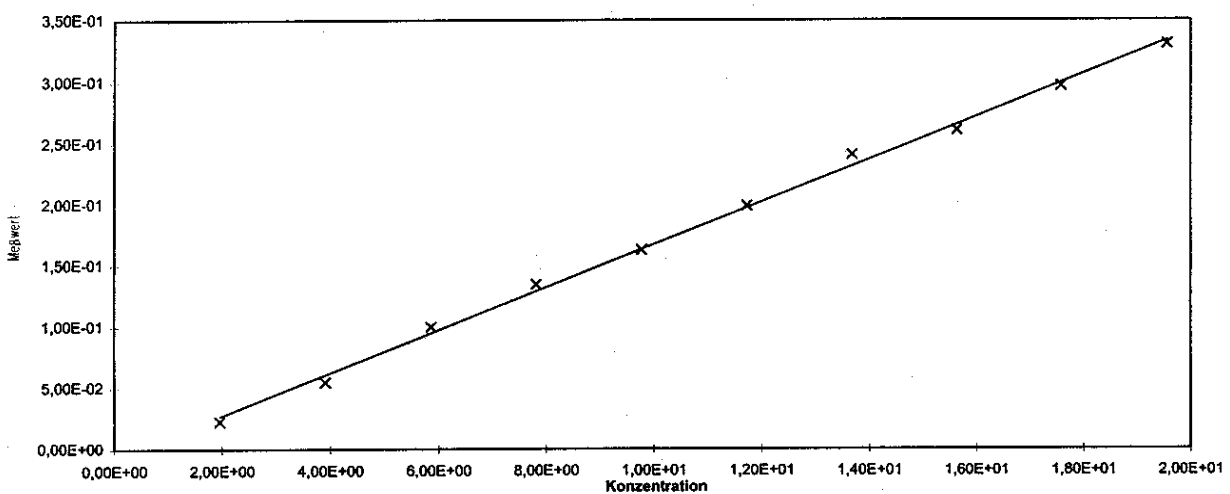

KAL_ZNMI:XLS

Ermittlung der Kalibrierfunktion

und der Verfahrenskenndaten

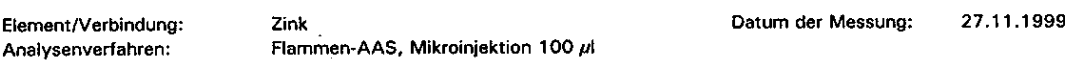

\section{Kenndaten der linearen Regression:}

Steigung:

y-Achsabschnitt:

Reststandardabweichung:

Verfahrensstandardabweichung:

Verfahrensvariationskoeffizient:

Prütgrößen:

$b=\quad 1,307 E+02$

$a=\quad 1,637 E+00$

$S_{y}=\quad 4,448 E+00$

$S_{x 0}=\quad 3,403 E-02$

$V_{x 0}=\quad 3,66 \%$

$Y_{p}=\quad 1,123 E+01$

Pearsonscher Korrelationskoeffizient:

$123 \mathrm{E}+01$
$1,448 \mathrm{E}-01$

$X_{\mathrm{p}} / x_{1}=\quad 144,8 \%$

$r=\quad 9,987 \mathrm{E}-01$

Graphische Darstellung der linearen Kalibrierfunktion:

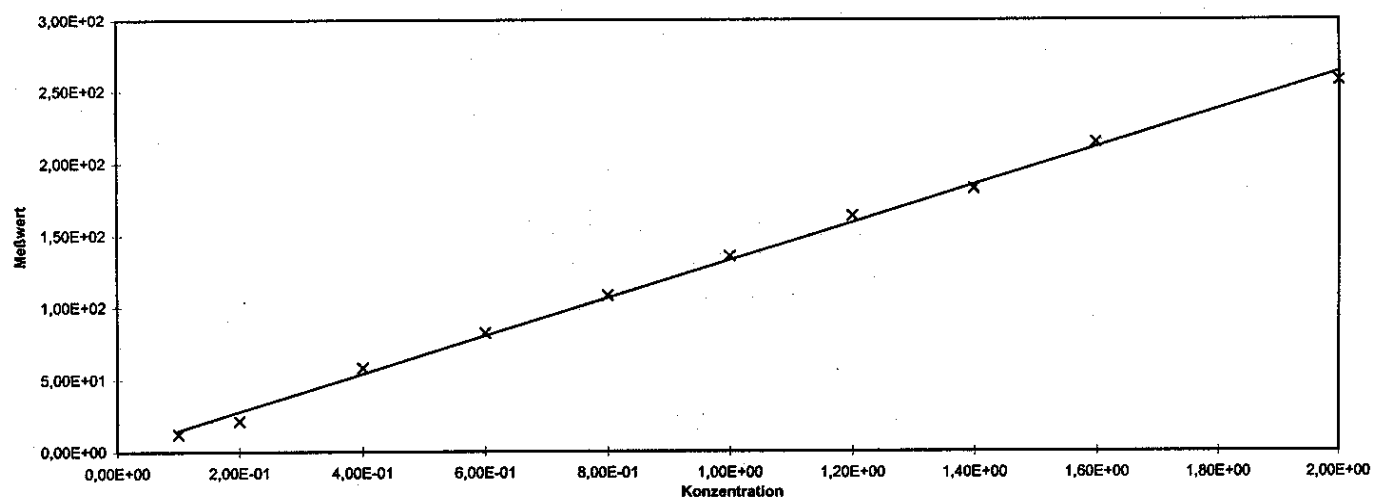




\section{Anhang 4:}

Einzelmeßwerte der Schwermetallbestimmungen $[\mu \mathrm{g} / \mathrm{g} \mathrm{TS}]$ in Organproben der Zwergfledermaus (Pipistrellus pipistrellus)

Blei:

a) Totfunde Harz:

\begin{tabular}{|c|c|c|c|c|c|c|}
\hline Tier-Nr. & Geschlecht & Alter & Herkunft & Lunge & Leber & Nieren \\
\hline 89006 & w & adult & Lerbach, LK Osterode a.H. & 1 & 9,8 & 2 \\
\hline 97001 & $\mathrm{~m}$ & adult & Lerbach, LK Osterode a.H. & 2,8 & 2,6 & 7,1 \\
\hline 98001 & w & adult & Osterode-OT Freiheit & 0,62 & 7,9 & 3,9 \\
\hline 98008 & w & juvenil & Steina, LK Osterode a.H. & 0,65 & 3,8 & 3,5 \\
\hline 98013 & $\mathrm{~m}$ & adult & Osterode-OT Freiheit & 0,22 & 5,1 & 2,7 \\
\hline 98014 & w & adult & Barbis, LK Osterode a.H. & 4,8 & 9,3 & 2,6 \\
\hline 98016 & $\mathrm{~m}$ & subadult & Osterode-Freiheit & 2,5 & 9,2 & 7,3 \\
\hline 98019 & $\mathrm{~m}$ & adult & Bad Sachsa, LK Osterode a.H. & 0,62 & 6,3 & 1,6 \\
\hline 98022 & $\mathrm{~m}$ & adult & Osterode-OT Förste & 8,67 & 3,2 & 1,1 \\
\hline 98023 & $\mathrm{~m}$ & adult & Osterode-OT Freiheit & & & 3,7 \\
\hline 98069 & $\mathrm{~m}$ & adult & Osterode, Stadtgebiet & & & 1,7 \\
\hline 98072 & $\mathrm{~m}$ & adult & Osterode, Stadtgebiet & & & 2,1 \\
\hline
\end{tabular}

b) harzferner Bereich:

\begin{tabular}{ccclccc}
\hline Tier-Nr. & Geschlecht & Alter & \multicolumn{1}{c}{ Herkunft } & Lunge & Leber & Nieren \\
\hline 90013 & m & juvenil & Lindau, LK Northeim & 0,97 & 1,3 & 0,65 \\
94040 & m & adult & Dransfeld, LK Göttingen & 0,59 & 1,1 & 1,4 \\
95016 & w & adult & Hammenstedt, LK Northeim & 0,9 & 3,7 & 1 \\
96023 & w & subadult & Rhumspringe, LK Göttingen & 0,8 & 1,2 & 0,53 \\
96026 & m & subadult & Hannover, Stadtgebiet & 0,62 & 10 & 0,6 \\
96028 & $?$ & subadult & Bothel, LK Rothenburg/W. & 0,35 & 2 & 1,5 \\
96029 & m & adult & Hannover, Stadtgebiet & 0,9 & 1,7 & 1,3 \\
97012 & m & adult & Marke, LK Osterode a.H. & & 6,7 & 0,74 \\
98088 & w & adult & Salzgitter-Bruchmachtersen & & 4,2 & 2,7 \\
98092 & w & adult & Bremen-Lilienthal & & 2,1 \\
\hline
\end{tabular}

Cadmium:

a) Totfunde Harz:

\begin{tabular}{ccclcc}
\hline Tier-Nr. & Geschlecht & Alter & Herkunft & Leber & Nieren \\
\hline 89006 & w & adult & Lerbach, LK Osterode a.H. & 0,32 & 0,29 \\
97001 & m & adult & Lerbach, LK Osterode a.H. & 2,8 & 7,7 \\
98001 & w & adult & Osterode-OT Freiheit & 1,0 & 0,81 \\
98008 & w & juvenil & Steina, LK Osterode a.H. & $<0,17$ & $<0,17$ \\
98013 & m & adult & Osterode-OT Freiheit & 5,5 & 4,4 \\
98014 & w & adult & Barbis, LK Osterode a.H. & 0,052 & 0,10 \\
98016 & m & subadult & Osterode-Freiheit & 6,5 & 14 \\
98019 & m & adult & Bad Sachsa, LK Osterode a.H. & 0,049 & 0,10 \\
98022 & m & adult & Osterode-OT Förste & 0,053 & $<0,09$ \\
98023 & m & adult & Osterode-OT Freiheit & 4,0 \\
98069 & m & adult & Osterode, Stadtgebiet & 0,17 & 0,25 \\
98072 & m & adult & Osterode, Stadtgebiet & 0,19 \\
\hline
\end{tabular}

b) harzferner Bereich:

\begin{tabular}{ccclcc}
\hline Tier-Nr. & Geschlecht & Alter & Herkunft & Leber & Nieren \\
\hline 90013 & m & juvenil & Lindau, LK Northeim & 0,19 & 0,79 \\
94040 & m & adult & Dransfeld, LK Göttingen & 0,81 & 2,1 \\
95016 & w & adult & Hammenstedt, LK Northeim & 1,4 & 2,7 \\
96023 & w & subadult & Rhumspringe, LK Göttingen & $<0,1$ & 0,059 \\
96026 & m & subadult & Hannover, Stadtgebiet & 0,12 & 0,50 \\
96028 & $?$ & subadult & Bothel, LK Rothenburg/W. & $<0,3$ & 0,17 \\
96029 & m & adult & Hannover, Stadtgebiet & 0,14 & 0,27 \\
97012 & m & adult & Marke, LK Osterode a.H. & 0,86 & 0,79 \\
98088 & w & adult & Salzgitter-Bruchmachtersen & 6,3 & 8,4 \\
98092 & w & adult & Bremen-Lilienthal & 1,4 & 0,72 \\
\hline
\end{tabular}


Chrom, ges.:

a) Totfunde Harz:

\begin{tabular}{ccclcc}
\hline Tier-Nr. & Geschlecht & Alter & \multicolumn{1}{c}{ Herkunft } & Leber & Nieren \\
\hline 89006 & w & adult & Lerbach, LK Osterode a.H. & 0,60 & $<0,1$ \\
97001 & m & adult & Lerbach, LK Osterode a.H. & $<0,07$ & 0,078 \\
98001 & w & adult & Osterode-OT Freiheit & 0,032 & 0,057 \\
98008 & w & juvenil & Steina, LK Osterode a.H. & 0,090 & 0,033 \\
98013 & m & adult & Osterode-OT Freiheit & 0,026 & $<0,05$ \\
98014 & w & adult & Barbis, LK Osterode a.H. & 0,17 & 1,7 \\
98016 & m & subadult & Osterode-Freiheit & $<0,04$ & 0,050 \\
98019 & m & adult & Bad Sachsa, LK Osterode a.H. & 0,049 & 0,081 \\
98022 & m & adult & Osterode-OT Förste & $<0,06$ & 0,22 \\
98023 & m & adult & Osterode-OT Freiheit & & 0,042 \\
98069 & $\mathrm{~m}$ & adult & Osterode, Stadtgebiet & $<0,06$ & $<0,1$ \\
98072 & $\mathrm{~m}$ & adult & Osterode, Stadtgebiet & & 0,090 \\
\hline
\end{tabular}

b) harzferner Bereich:

\begin{tabular}{ccclcc}
\hline Tier-Nr. & Geschlecht & Alter & Herkunft & Leber & Nieren \\
\hline 90013 & m & juvenil & Lindau, LK Northeim & 0,15 & 1,4 \\
94040 & m & adult & Dransfeld, LK Göttingen & 0,026 & $<0,08$ \\
95016 & w & adult & Hammenstedt, LK Northeim & 0,039 & $<0,08$ \\
96023 & w & subadult & Rhumspringe, LK Göttingen & $<0,1$ & $<0,1$ \\
96026 & m & subadult & Hannover, Stadtgebiet & $<0,05$ & 0,58 \\
96028 & $?$ & subadult & Bothel, LK Rothenburg/W. & $<0,28$ & $<0,3$ \\
96029 & m & adult & Hannover, Stadtgebiet & 0,090 & 0,18 \\
97012 & m & adult & Marke, LK Osterode a.H. & 0,038 & 0,29 \\
98088 & w & adult & Salzgitter-Bruchmachtersen & 0,10 & 0,081 \\
98092 & w & adult & Bremen-Lilienthal & 0,069 & 0,20 \\
\hline
\end{tabular}


Anhang 5: Detailangaben zu den untersuchten Tieren und Auflistung der Meßwerte

\begin{tabular}{|c|c|c|c|c|c|c|c|c|c|c|c|c|c|c|c|c|c|}
\hline $\begin{array}{l}\text { Tier } \\
\text { Nr. }\end{array}$ & Art & Fundort & Geschl. & Alter & Funddatum & \begin{tabular}{|c|} 
Blatt \\
Nr. \\
TK 25
\end{tabular} & Quadr. & $\begin{array}{c}\text { Körper } \\
\text { Gew. } \\
\text { [g] }\end{array}$ & $\begin{array}{c}\text { Körper } \\
\text { länge } \\
{[\mathrm{cm}]}\end{array}$ & $\begin{array}{c}\text { Unter- } \\
\text { arm- } \\
\text { länge } \\
\text { [cm] }\end{array}$ & $\begin{array}{c}\mathrm{Pb} \\
\text { Haare } \\
{[\mu \mathrm{g} / \mathrm{g}]}\end{array}$ & $\begin{array}{c}\text { Cd } \\
\text { Haare } \\
{[\mu \mathrm{g} / \mathrm{g}]}\end{array}$ & $\begin{array}{c}\mathrm{Cr} \\
\text { Haare } \\
{[\mu \mathrm{g} / \mathrm{g}]}\end{array}$ & $\begin{array}{c}\mathrm{Cu} \\
\text { Haare } \\
{[\mu \mathrm{g} / \mathrm{g}]}\end{array}$ & $\begin{array}{c}\mathrm{Ni} \\
\text { Haare } \\
{[\mu \mathrm{g} / \mathrm{g}]}\end{array}$ & $\begin{array}{c}\mathrm{Zn} \\
\text { Haare } \\
{[\mu \mathrm{g} / \mathrm{g}]}\end{array}$ & $\begin{array}{c}\mathrm{Pb} \\
\text { Kno- } \\
\text { chen } \\
{[\mu \mathrm{g} / \mathrm{g}} \\
\mathrm{TS}]\end{array}$ \\
\hline 87001 & Pipistrellus pipistrellus - Zwergfledermaus & Eisdorf, Stadt Osterode a.H. & $\mathrm{m}$ & adult & $2 / 87$ & 4227 & 3 & 1,63 & 4,8 & 3,19 & 83 & 1,6 & 4,6 & 32 & 6,6 & 440 & 25 \\
\hline 87002 & Pipistrellus pipistrellus - Zwergfledermaus & Landolfshausen, LK Göttingen & $\mathrm{m}$ & adult & 22.8.1987 & 4426 & 4 & 1,52 & 3,9 & 2,99 & 18 & 0,72 & 3,1 & 8,6 & 4,0 & 260 & 9,7 \\
\hline 87004 & Pipistrellus pipistrellus - Zwergfledermaus & Osterode a.H., Gipsstollen & w & adult & 3.1987 & 4227 & 4 & 1,56 & 3,7 & 3,12 & 97 & 1,4 & 5,6 & 48 & 4,2 & 650 & 7,8 \\
\hline 87010 & Pipistrellus pipistrellus - Zwergfledermaus & Barsinghausen & w & subad. & 8.8.1987 & 3622 & 4 & 1,14 & 3,4 & 3,00 & 25 & 0,11 & 4,6 & 18 & 2,4 & 240 & 4,2 \\
\hline 87011 & Pipistrellus pipistrellus - Zwergfledermaus & Bad Pyrmont & w & adult & 2.4.1987 & 4021 & 1 & 2,92 & 4,0 & 3,12 & 50 & 0,66 & 1,1 & 11 & 2,3 & 270 & \\
\hline 87013 & Myotis myotis - Gr. Mausohr & Greene, LK Northeim & $\mathrm{m}$ & adult & Aug. 1987 & 4125 & 2 & 5,50 & 6,3 & 5,55 & & & & & & & 25 \\
\hline 87015 & Pipistrellus pipistrellus - Zwergfledermaus & Celle & $\mathrm{m}$ & adult & & 3326 & 3 & 1,47 & 3,9 & 3,10 & 86 & 1,8 & 2,5 & 40 & 6,2 & 660 & 50 \\
\hline 87016 & Pipistrellus pipistrellus - Zwergfledermaus & Cuxhafen & w & adult & 3.9 .1987 & 2117 & 1 & 1,67 & 4,4 & 3,24 & 100 & 0,28 & 1,9 & 13 & 3,0 & 250 & 18 \\
\hline 87018 & Pipistrellus pipistrellus - Zwergfledermaus & Reinerbeck bei Hameln & w & adult & 18.7.1987 & 3921 & 3 & 1,70 & 3,5 & 2,97 & 5,9 & 0,17 & 0,97 & 10 & 4,7 & 220 & 6,5 \\
\hline 88001 & Myotis myotis - Gr. Mausohr & Spinnerei Hattorf, LK Osterode & w & adult & 23.10 .1998 & 4327 & 3 & & & & & & & & & & 30 \\
\hline 88002 & Myotis myotis - Gr. Mausohr & Spinnerei Hattorf, LK Osterode & w & adult & 23.10 .1998 & 4327 & 3 & & & & & & & & & & 14 \\
\hline 88003 & Myotis myotis - Gr. Mausohr & Spinnerei Hattorf, LK Osterode & w & adult & 23.10 .1998 & 4327 & 3 & & & & & & & & & & 20 \\
\hline 88004 & Myotis myotis - Gr. Mausohr & Spinnerei Hattorf, LK Osterode & w & adult & 23.10 .1998 & 4327 & 3 & & & & & & & & & & 27 \\
\hline 88005 & Myotis myotis - Gr. Mausohr & Spinnerei Hattorf, LK Osterode & w & adult & 23.10 .1998 & 4327 & 3 & & & & & & & & & & 16 \\
\hline 88008 & Pipistrellus pipistrellus - Zwergfledermaus & Nienstedt, Stadt Osterode a.H. & $\mathrm{m}$ & adult & 10.8.1988 & 4227 & 3 & 2,04 & 3,7 & 3,12 & & & & & & & 59 \\
\hline 88009 & Pipistrellus pipistrellus - Zwergfledermaus & Braunschweig & $\mathrm{m}$ & adult & 7.4 .1988 & 3729 & 1 & 1,24 & 4,0 & 3,00 & 170 & 1,0 & 0,90 & 18 & 3,6 & 330 & 21 \\
\hline 88015 & Pipistrellus pipistrellus - Zwergfledermaus & Wunstorf & w & juv. & 9.1988 & 3522 & 4 & 0,99 & 2,9 & 2,73 & 22 & 0,54 & 4,3 & 150 & 4,0 & 290 & 5,5 \\
\hline 88017 & Myotis myotis - Gr. Mausohr & Spinnerei Hattorf, LK Osterode & w & adult & 23.10 .1998 & 4327 & 3 & & & & & & & & & & 13 \\
\hline 88018 & Myotis myotis - Gr. Mausohr & Spinnerei Hattorf, LK Osterode & w & adult & 23.10 .1998 & 4327 & 3 & & & & & & & & & & 15 \\
\hline 88019 & Myotis myotis - Gr. Mausohr & Spinnerei Hattorf, LK Osterode & w & adult & 23.10 .1998 & 4327 & 3 & & & & & & & & & & 12 \\
\hline 88020 & Myotis myotis - Gr. Mausohr & Spinnerei Hattorf, LK Osterode & w & adult & 23.10 .1998 & 4327 & 3 & & & & & & & & & & 21 \\
\hline 88021 & Myotis myotis - Gr. Mausohr & Spinnerei Hattorf, LK Osterode & w & adult & 23.10 .1998 & 4327 & 3 & & & & & & & & & & 24 \\
\hline 89004 & Pipistrellus pipistrellus - Zwergfledermaus & Stadt Osterode a.H. & $\mathrm{m}$ & adult & 1.12 .1989 & 4227 & 4 & 4,35 & 4,4 & 3,20 & 27 & 0,22 & 2,0 & 12 & 1,0 & 400 & 23 \\
\hline 89006 & Pipistrellus pipistrellus - Zwergfledermaus & Lerbach, Stadt Osterode a.H. & w & adult & 10.1989 & 4227 & 2 & 5,10 & 4,2 & 3,35 & 37 & 0,38 & 1,2 & 46 & 2,4 & 270 & 42 \\
\hline 89009 & Pipistrellus pipistrellus - Zwergfledermaus & Stadt Osterode a.H. & $\mathrm{m}$ & subad. & 1989 & 4227 & 4 & 2,11 & 3,1 & 3,09 & 100 & 0,46 & 2,1 & 24 & 1,0 & 280 & 9,1 \\
\hline 90001 & Pipistrellus pipistrellus - Zwergfledermaus & Hedemünden, nahe Werra & $\mathrm{m}$ & adult & 5.6 .1990 & 4624 & 2 & 2,37 & 4,0 & 3,19 & 61 & 0,43 & 1,2 & 22 & 3,6 & 290 & 21 \\
\hline 90002 & Pipistrellus pipistrellus - Zwergfledermaus & Lüneburg & w & adult & 22.6.1990 & 2728 & 3 & 4,40 & 3,7 & 3,12 & 360 & 0,37 & 0,96 & 17 & 1,4 & 400 & 79 \\
\hline 90005 & Pipistrellus pipistrellus - Zwergfledermaus & Bleckede, bei Lüneburg & $\mathrm{m}$ & adult & 13.6.1990 & 2730 & 1 & 1,82 & 3,7 & 3,06 & 94 & 0,17 & 3,7 & 134 & 2,3 & 260 & 8,5 \\
\hline 90007 & Pipistrellus pipistrellus - Zwergfledermaus & Hannover, Stadt & $\mathrm{m}$ & adult & 31.8 .1990 & 3624 & 1 & 3,12 & 3,8 & 3,12 & 44 & 0,34 & 1,4 & 13 & 1,5 & 240 & 19 \\
\hline 90009 & Pipistrellus pipistrellus - Zwergfledermaus & Förste, Stadt Osterode a.H. & w & adult & 13.5 .1990 & 4227 & 3 & 4,61 & 4,1 & 3,25 & 52 & 0,57 & 2,6 & 15 & 1,2 & 230 & 1,4 \\
\hline 90010 & Pipistrellus pipistrellus - Zwergfledermaus & Eisdorf, Stadt Osterode a.H & w & juv. & 9.7 .1990 & 4227 & 3 & 1,96 & 3,3 & 2,65 & & & & & & & 9,9 \\
\hline 90012 & Pipistrellus pipistrellus - Zwergfledermaus & Schneverdingen, Norddt. Natur- & w & adult & 15.6.1990 & 2824 & 4 & 2,88 & 4,2 & 3,16 & 180 & 0,41 & 1,8 & 16 & 2,9 & 390 & 44 \\
\hline 90013 & Pipistrellus pipistrellus - Zwergfledermaus & Lindau, LK Northeim & $\mathrm{m}$ & juv. & 25.6.1990 & 4326 & 2 & 2,62 & 3,7 & 3,10 & 23 & 0,55 & 3,9 & 17 & 2,4 & 320 & 3,7 \\
\hline 91002 & Pipistrellus pipistrellus - Zwergfledermaus & Eltze, Peiner Str. $19 \mathrm{~A}$ & $\mathrm{~m}$ & adult & 22.7.1991 & 3527 & 2 & 0,95 & 4,1 & 3,10 & 36 & 7,9 & 2,1 & 20 & 4,8 & 380 & 9,6 \\
\hline 91019 & Pipistrellus pipistrellus - Zwergfledermaus & Lüneburg, St. Michaelis Kirche & $\mathrm{m}$ & juv. & 18.9.1991 & 2728 & 3 & 0,78 & 3,0 & 2,39 & 103 & 0,11 & 2,8 & 83 & 0,83 & 170 & 34 \\
\hline 91021 & Pipistrellus pipistrellus - Zwergfledermaus & Nienstedt, Stadt Osterode a.H & $\mathrm{m}$ & juv. & 15.7.1991 & 4227 & 3 & & & 2,28 & 45 & 0,24 & 8,6 & 83 & 4,0 & 180 & 48 \\
\hline 92001 & Pipistrellus pipistrellus - Zwerafledermaus & Amelinahausen & $\mathrm{m}$ & adult & 31.8 .1992 & 2827 & 3 & 4.55 & 4.6 & 3.08 & 14 & 0.42 & 5.3 & 9.5 & 1.9 & 290 & 9.5 \\
\hline 92004 & Pipistrellus nathusii - Rauhhautfledermaus & Marienhafe, LK Aurich & w & adult & 23.6.1992 & 2409 & 4 & 8,40 & 4,5 & 3,47 & & & & & & & 17 \\
\hline
\end{tabular}




\begin{tabular}{|c|c|c|c|c|c|c|c|c|c|c|c|c|c|c|c|c|c|}
\hline $\begin{array}{l}\text { Tier } \\
\text { Nr. }\end{array}$ & Art & Fundort & Geschl. & Alter & Funddatum & \begin{tabular}{|c|} 
Blatt \\
Nr. \\
TK 25
\end{tabular} & Quadr. & $\begin{array}{c}\text { Körper } \\
\text { Gew. } \\
\text { [g] }\end{array}$ & $\begin{array}{c}\text { Körper } \\
\text { länge } \\
\text { [cm] }\end{array}$ & $\begin{array}{c}\text { Unter- } \\
\text { arm- } \\
\text { länge } \\
\text { [cm] }\end{array}$ & \begin{tabular}{|l|}
$\mathrm{Pb}$ \\
Haare \\
{$[\mu \mathrm{g} / \mathrm{g}]$}
\end{tabular} & $\begin{array}{c}\text { Cd } \\
\text { Haare } \\
{[\mu \mathrm{g} / \mathrm{g}]}\end{array}$ & \begin{tabular}{|c|}
$\mathrm{Cr}$ \\
Haare \\
{$[\mu \mathrm{g} / \mathrm{g}]$}
\end{tabular} & $\begin{array}{l}\mathrm{Cu} \\
\text { Haare } \\
{[\mu \mathrm{g} / \mathrm{g}]}\end{array}$ & $\begin{array}{c}\mathrm{Ni} \\
\text { Haare } \\
{[\mu \mathrm{g} / \mathrm{g}]}\end{array}$ & \begin{tabular}{|c|}
$\mathrm{Zn}$ \\
Haare \\
{$[\mu \mathrm{g} / \mathrm{g}]$} \\
\end{tabular} & $\begin{array}{r}\mathrm{Pb} \\
\text { Kno- } \\
\text { chen } \\
{[\mu \mathrm{g} / \mathrm{g}} \\
\mathrm{TS}]\end{array}$ \\
\hline 92005 & Pipistrellus nathusii - Rauhhautfledermaus & Marienhafe, LK Aurich & w & adult & 23.6 .1992 & 2409 & 4 & 9,72 & 4,1 & 3,44 & & & & & & & 17 \\
\hline 92055 & Pipistrellus pipistrellus - Zwergfledermaus & Göttingen, Stadtmauer & $\mathrm{m}$ & adult & 23.4.1992 & 4425 & 4 & 1,85 & 3,8 & 3,08 & 94 & 0,42 & 1,0 & 23 & 3,2 & 430 & 10 \\
\hline 93001 & Pipistrellus pipistrellus - Zwergfledermaus & Wunstorf & w & adult & 3.12 .1993 & 3522 & 4 & 1,64 & 3,9 & 3,07 & 110 & 1,1 & 3,6 & 24 & 4,0 & 500 & 26 \\
\hline 93003 & Myotis myotis - Gr. Mausohr & Greene, LK Northeim & $\mathrm{m}$ & subad. & 23.6.1993 & 4125 & 2 & 14,41 & 5,1 & 4,43 & & & & & & & 9,2 \\
\hline 93005 & Myotis myotis - Gr. Mausohr & Greene, LK Northeim & w & subad. & 23.6.1993 & 4125 & 2 & 10,10 & 4,5 & 4,25 & & & & & & & 5,3 \\
\hline 93006 & Myotis myotis - Gr. Mausohr & Greene, LK Northeim & $\mathrm{m}$ & subad. & 23.6.1993 & 4125 & 2 & 7,94 & 4,8 & 3,85 & & & & & & & 3,9 \\
\hline 93007 & Myotis myotis - Gr. Mausohr & Greene, LK Northeim & w & subad. & 23.6 .1993 & 4125 & 2 & 6,50 & 4,6 & 3,52 & & & & & & & 2,7 \\
\hline 93008 & Myotis myotis - Gr. Mausohr & Greene, LK Northeim & w & juv. & 23.6 .1993 & 4125 & 2 & 6,89 & 4,3 & 3,08 & & & & & & & 2,7 \\
\hline 93009 & Myotis myotis - Gr. Mausohr & Greene, LK Northeim & w & subad. & 23.6 .1993 & 4125 & 2 & 9,05 & 5,5 & 4,13 & & & & & & & 3,4 \\
\hline 93010 & Myotis myotis - Gr. Mausohr & Greene, LK Northeim & $\mathrm{m}$ & subad. & 23.6.1993 & 4125 & 2 & 10,98 & 5,1 & 4,77 & & & & & & & 5,5 \\
\hline 93011 & Myotis myotis - Gr. Mausohr & Greene, LK Northeim & $\mathrm{m}$ & subad. & 23.6 .1993 & 4125 & 2 & 8,78 & 5,2 & 4,12 & & & & & & & 4,7 \\
\hline 93012 & Myotis myotis - Gr. Mausohr & Greene, LK Northeim & $\mathrm{m}$ & subad. & 23.6 .1993 & 4125 & 2 & 8,92 & 4,7 & 3,93 & & & & & & & 3,9 \\
\hline 93013 & Myotis myotis - Gr. Mausohr & Greene, LK Northeim & w & juv. & 23.6.1993 & 4125 & 2 & 8,21 & 4,8 & 3,51 & & & & & & & 2,8 \\
\hline 93014 & Myotis myotis - Gr. Mausohr & Greene, LK Northeim & w & subad. & 23.6 .1993 & 4125 & 2 & 8,02 & 4,1 & 3,98 & & & & & & & 3,7 \\
\hline 93015 & Myotis myotis - Gr. Mausohr & Greene, LK Northeim & w & juv. & 23.6.1993 & 4125 & 2 & 6,47 & 4,0 & 3,20 & & & & & & & 8 \\
\hline 93016 & Myotis myotis - Gr. Mausohr & Greene, LK Northeim & w & juv. & 23.6 .1993 & 4125 & 2 & 4,82 & 3,3 & 2,61 & & & & & & & 6,1 \\
\hline 93052 & Pipistrellus pipistrellus - Zwergfledermaus & Hannover & $\mathrm{m}$ & adult & 26.12 .93 & 3624 & 1 & 3,22 & 4,1 & 3,05 & 42 & 0,31 & 1,7 & 13 & 1,3 & 236 & 5,5 \\
\hline 93056 & Pipistrellus pipistrellus - Zwergfledermaus & Hellwege & w & juv. & 07.93 & 2921 & 1 & 1,66 & 4,0 & 2,30 & & & & & & & 14 \\
\hline 93058 & Pipistrellus pipistrellus - Zwergfledermaus & Volkmarshausen & w & adult & $30.11 .93 ?$ & 4523 & 4 & 3,90 & 3,5 & 3,02 & 51 & 0,47 & 4,0 & 57 & 2,5 & 340 & 6,9 \\
\hline 94002 & Pipistrellus pipistrellus - Zwergfledermaus & Hann.-Münden, Stadtmitte & $\mathrm{m}$ & adult & 2.11 .1994 & 4523 & 4 & 2,72 & 3,7 & 3,36 & 84 & 0,61 & 2,5 & 31 & 2,5 & 460 & 7,5 \\
\hline 94004 & Pipistrellus pipistrellus - Zwergfledermaus & Badenhausen & $\mathrm{m}$ & adult & 14.2.94 & 4227 & 1 & 4,55 & 4,2 & 3,15 & 31 & 0,48 & 2 & 22 & 1,6 & 240 & 8,2 \\
\hline 94011 & Pipistrellus pipistrellus - Zwergfledermaus & Eisdorf, Stadt Osterode a.H. & $\mathrm{m}$ & subad. & 23.7 .94 & 4227 & 3 & 3,25 & 3,7 & 3,08 & 7,2 & 0,11 & 1,7 & 25 & 1,2 & 230 & 6,4 \\
\hline 94015 & Pipistrellus pipistrellus - Zwergfledermaus & Förste, Stadt Osterode a.H. & w & adult & 24.4 .94 & 4227 & 3 & 3,31 & 4,0 & 3,14 & 37 & 0,28 & 1,7 & 24 & 1,5 & 970 & 23 \\
\hline 94017 & Pipistrellus pipistrellus - Zwergfledermaus & Förste, Stadt Osterode a.H. & w & subad. & 26.7 .94 & 4227 & 3 & 2,68 & 3,8 & 3,18 & 6 & 0,09 & 0,75 & 38 & 1,2 & 200 & 5,2 \\
\hline 94034 & Pipistrellus pipistrellus - Zwergfledermaus & Elbingerode & $\mathrm{m}$ & subad. & 21.4 .94 & 4327 & 2 & 2,21 & 3,7 & 2,95 & 40 & 0,49 & 9,4 & 35 & 1,8 & 720 & 4,4 \\
\hline 94039 & Pipistrellus pipistrellus - Zwergfledermaus & Varmissen & $\mathrm{m}$ & adult & 21.7 .94 & 4424 & 4 & 1,61 & 3,6 & 3,14 & 8,4 & 0,17 & 16 & 24 & 1,2 & 330 & 4,9 \\
\hline 94040 & Pipistrellus pipistrellus - Zwergfledermaus & Hägerhof (Dransfeld) & $\mathrm{m}$ & adult & 8.5 .94 & 4524 & 2 & 3,35 & 4,1 & 3,11 & 13 & 0,14 & 0,96 & 41 & 1,8 & 240 & 70 \\
\hline 95001 & Myotis natteri - Fransenfledermaus & Rössing, Gde. Nordstemmen, & $\mathrm{m}$ & adult & 10.10 .1995 & 3824 & 2 & 6,82 & 4,8 & 4,03 & 1,8 & 0,03 & 1,1 & 8,7 & 0,80 & 120 & 1,7 \\
\hline 95009 & Pipistrellus pipistrellus - Zwergfledermaus & Hachenhausen Landkreis NOM & $\mathrm{m}$ & adult & 5.7 .95 & 4126 & 1 & 2,30 & 4,0 & 3,16 & 320 & 6,5 & 3,1 & 35 & 2,4 & 690 & 5,5 \\
\hline 95010 & Pipistrellus pipistrellus - Zwergfledermaus & Schwiegershsn., Osterode a.H. & w & subad. & 16.7 .95 & 4327 & 1 & 2,05 & 3,4 & 3,10 & 9,6 & 0,16 & 1,2 & 32 & 2,9 & 260 & 9,2 \\
\hline 95012 & Pipistrellus pipistrellus - Zwergfledermaus & Osterode am Harz & w & adult & 6.9 .95 & 4227 & 4 & 2,34 & 3,7 & 2,95 & 39 & 0,47 & 2,7 & 33 & 4,1 & 280 & 21 \\
\hline 95014 & Pipistrellus pipistrellus - Zwergfledermaus & Ührde, Osterode a.H. & $\mathrm{m}$ & adult & 25.6 .95 & 4227 & 3 & 3,11 & 3,8 & 2,97 & 42 & 1,36 & 2,5 & 26 & 2,1 & 1100 & 7,5 \\
\hline 95016 & Pipistrellus pipistrellus - Zwergfledermaus & Hammenstedt Landkreis NOM & w & adult & 8.7 .95 & 4326 & 1 & 4,38 & 4,2 & 3,77 & 6,8 & 0,15 & 1,1 & 19 & 0,53 & 220 & 11 \\
\hline 95017 & Pipistrellus pipistrellus - Zwergfledermaus & Hammenstedt Landkreis NOM & w & juv. & 8.7 .95 & 4326 & 1 & 1,49 & 2,4 & 2,18 & & & & & & & 0,98 \\
\hline 95018 & Pipistrellus pipistrellus - Zwergfledermaus & Hammenstedt Landkreis NOM & $\mathrm{m}$ & juv. & 8.7 .95 & 4326 & 1 & 1,32 & 2,4 & 1,99 & & & & & & & $<0,5$ \\
\hline 95020 & Pipistrellus pipistrellus - Zwergfledermaus & Hannover, Schulenburg & $\mathrm{m}$ & adult & 23.8 .95 & 3524 & 4 & 2,08 & 3,9 & 3,03 & 47 & 8,4 & 3,5 & 31 & 2,0 & 330 & 53 \\
\hline 95021 & Pipistrellus pipistrellus - Zwergfledermaus & Förste, Stadt Osterode a.H & $\mathrm{m}$ & adult & 19.10 .95 & 4227 & 3 & 2,50 & 4,0 & 3,17 & 82 & 0,35 & 1,3 & 20 & 0,82 & 590 & 52 \\
\hline 96001 & Pipistrellus pipistrellus - Zwergfledermaus & Hannover-Kleefeld & $\mathrm{m}$ & adult & 11.8 .96 & 3624 & 2 & 3,80 & 4,8 & 3,10 & 64 & 1,1 & 2,6 & 16 & 2,6 & 1500 & 7,3 \\
\hline 96002 & Pipistrellus pipistrellus - Zwergfledermaus & Hannover, Rühmkorfstr. & $\mathrm{m}$ & adult & 22.8 .1996 & 3524 & 3 & 2,20 & 4,1 & 3,09 & 38 & 0,37 & 3,0 & 46 & 4,2 & 410 & 10 \\
\hline 96003 & Pipistrellus pipistrellus - Zwergfledermaus & Hannover, Rühmkorfstraße & $\mathrm{m}$ & adult & 22.8 .1996 & 3524 & 3 & 1,87 & 3,6 & 3,07 & 63 & 0,81 & 3,2 & 41 & 4,4 & 420 & 34 \\
\hline 96004 & Myotis mystacinus - Bartfledermaus & Harsum & w & adult & 10.8.1996 & 3725 & 4 & 3,53 & 4,8 & 3,52 & 1,7 & 0,08 & 1,1 & 6,9 & 0,83 & 220 & 7,7 \\
\hline 96005 & Plecotus austriacus - Graues Langohr & Schnackenburg & $\mathrm{m}$ & adult & $3 / 1996$ & 2935 & 3 & 5,64 & 3,6 & 4,00 & & & & & & & 7,9 \\
\hline
\end{tabular}




\begin{tabular}{|c|c|c|c|c|c|c|c|c|c|c|c|c|c|c|c|c|c|}
\hline $\begin{array}{l}\text { Tier } \\
\text { Nr. }\end{array}$ & Art & Fundort & Geschl. & Alter & Funddatum & \begin{tabular}{|c|} 
Blatt \\
Nr. \\
TK 25
\end{tabular} & Quadr. & $\begin{array}{c}\text { Körper } \\
\text { Gew. } \\
\text { [g] }\end{array}$ & $\begin{array}{c}\text { Körper } \\
\text { länge } \\
\text { [cm] }\end{array}$ & $\begin{array}{c}\text { Unter- } \\
\text { arm- } \\
\text { länge } \\
\text { [cm] }\end{array}$ & $\begin{array}{l}\mathrm{Pb} \\
\text { Haare } \\
{[\mu \mathrm{g} / \mathrm{g}]}\end{array}$ & $\begin{array}{c}\mathrm{Cd} \\
\text { Haare } \\
{[\mu \mathrm{g} / \mathrm{g}]} \\
\end{array}$ & $\begin{array}{c}\mathrm{Cr} \\
\text { Haare } \\
{[\mu \mathrm{g} / \mathrm{g}]}\end{array}$ & $\begin{array}{l}\text { Cu } \\
\text { Haare } \\
{[\mu \mathrm{g} / \mathrm{g}]}\end{array}$ & $\begin{array}{c}\mathrm{Ni} \\
\text { Haare } \\
{[\mu \mathrm{g} / \mathrm{g}]}\end{array}$ & \begin{tabular}{|l|} 
Zn \\
Haare \\
{$[\mu \mathrm{g} / \mathrm{g}]$} \\
\end{tabular} & $\begin{array}{r}\mathrm{Pb} \\
\mathrm{Kno} \\
\mathrm{chen} \\
{[\mu \mathrm{g} / \mathrm{g}} \\
\mathrm{TS}]\end{array}$ \\
\hline 96016 & Pipistrellus pipistrellus - Zwergfledermaus & Kreiensen & $\mathrm{m}$ & subad. & 18.9.96 & 4125 & 2 & 2,86 & 3,8 & 3,06 & 140 & 0,74 & 1,8 & 51 & 1,6 & 300 & \\
\hline 96021 & Pipistrellus pipistrellus - Zwergfledermaus & Greene Landkreis NOM & w & adult & 12.6.96 & 4125 & 2 & 3,43 & 3,9 & 3,20 & 120 & 1,62 & 4 & 39 & 4,1 & 420 & 30 \\
\hline 96023 & Pipistrellus pipistrellus - Zwergfledermaus & Rhumspringe Landkreis Gö. & w & subad. & 23.7.96 & 4427 & 2 & 2,43 & 3,8 & 3,02 & 14 & 0,25 & 5,4 & 59 & 2,7 & 230 & 8,0 \\
\hline 96026 & Pipistrellus pipistrellus - Zwergfledermaus & Hannover & $\mathrm{m}$ & subad. & 24.8.96 & 3624 & 1 & 3,68 & 4,1 & 2,98 & 26 & 0,3 & 2,0 & 13 & 1,6 & 270 & 4,9 \\
\hline 96027 & Pipistrellus pipistrellus - Zwergfledermaus & Hannover & w & subad. & 9.8 .96 & 3624 & 1 & 2,32 & 3,6 & 2,94 & 93 & 0,3 & 4,1 & 42 & 4,0 & 1200 & 7,4 \\
\hline 96028 & Pipistrellus pipistrellus - Zwergfledermaus & Bothel & - & subad. & & 2923 & 1 & 2,70 & 3,9 & 2,90 & 22 & 0,31 & 5,0 & 135 & 2,6 & 280 & 8,5 \\
\hline 96029 & Pipistrellus pipistrellus - Zwergfledermaus & Hannover & $\mathrm{m}$ & adult & 23.8.96 & 3624 & 1 & 4,50 & 3,6 & 3,16 & 23 & 0,27 & 1,4 & 28 & 3,7 & 270 & 11 \\
\hline 97001 & Pipistrellus pipistrellus - Zwergfledermaus & Osterode-Clausthal, B241 & $\mathrm{m}$ & adult & 6.11.1997 & 4227 & 2 & & & 3,21 & 43 & 0,76 & 1,9 & 13 & 1,8 & 580 & 28 \\
\hline 97012 & Pipistrellus pipistrellus - Zwergfledermaus & Marke Landkreis Osterode a.H. & $\mathrm{m}$ & adult & 1.8 .97 & 4226 & 4 & 4,51 & 4,3 & 3,11 & 8,0 & 0,16 & 0,51 & 30 & 3,2 & 240 & 9,0 \\
\hline 97013 & Pipistrellus pipistrellus - Zwergfledermaus & Elvershausen Landkreis NOM & $\mathrm{m}$ & subad. & 2.8 .97 & 4226 & 4 & 2,26 & 3,0 & 2,78 & 8,6 & 0,45 & 2,2 & 34 & 2,1 & 280 & 3,9 \\
\hline 97014 & Pipistrellus pipistrellus - Zwergfledermaus & Harriehausen Landkreis NOM & $\mathrm{m}$ & subad. & 6.7.97 & 4126 & 4 & 2,16 & 3,3 & 2,81 & 3,4 & $<0,4$ & 2,7 & 52 & $<0,04$ & 200 & 3,3 \\
\hline 98001 & Pipistrellus pipistrellus - Zwergfledermaus & Osterode a.H. - Ortsteil Freiheit & $\mathrm{m}$ & adult & 19.3.1998 & 4227 & 4 & 3,50 & & 3,12 & 61 & 0,35 & 2,4 & 13 & 1,4 & 230 & 8,3 \\
\hline 98002 & Myotis bechsteinii - Bechsteinfledermaus & Herzberg am Harz & w & adult & 29.6.1998 & 4328 & 1 & 10,38 & & 4,26 & 5,2 & 0,074 & 0,32 & 8,1 & 0,32 & 110 & \\
\hline 98003 & Pipistrellus pipistrellus - Zwergfledermaus & Schwiegershausen, Stadt Oster- & w & adult & 31.5 .1998 & 4327 & 1 & 3,58 & & 3,48 & 1,2 & 0,35 & 0,18 & 7,2 & 0,52 & 150 & 81 \\
\hline 98004 & Pipistrellus pipistrellus - Zwergfledermaus & Schwiegershausen, Stadt Oster- & $\mathrm{m}$ & adult & 31.5 .1998 & 4327 & 1 & 4,34 & & 3,40 & 10 & 0,069 & 0,15 & 6,4 & 0,37 & 110 & 45 \\
\hline 98005 & Pipistrellus pipistrellus - Zwergfledermaus & Förste, Stadt Osterode a.H. & w & juv. & 22.7.1998 & 4227 & 3 & 2,05 & 3,5 & 2,54 & 16 & 0,25 & 3,0 & 34 & 1,6 & 210 & 13 \\
\hline 98006 & Myotis mystacinus - Bartfledermaus & Schwiegershausen, Stadt Oster- & $\mathrm{m}$ & adult & 2.7 .1998 & 4327 & 1 & 4,26 & 3,8 & 3,45 & 8,0 & 0,077 & 0,27 & 6,9 & 0,51 & 130 & \\
\hline 98007 & Pipistrellus pipistrellus - Zwergfledermaus & Steina/Bad Sachsa & w & juv. & 12.7.1998 & 4429 & 1 & 2,81 & 3,2 & 2,90 & 3,3 & 0,12 & & 9,6 & 0,97 & 370 & 22 \\
\hline 98008 & Pipistrellus pipistrellus - Zwergfledermaus & Steina/Bad Sachsa & w & juv. & 12.7.1998 & 4429 & 1 & 2,05 & 2,8 & 2,69 & 8,7 & 0,077 & 0,5 & 6,3 & 1,4 & 260 & 12 \\
\hline 98009 & Pipistrellus nathusii - Rauhhautfledermaus & Wulften bei Osterode a.H. & w & adult & 26.4.1998 & 4327 & 1 & 6,75 & 4,5 & 3,48 & 7,4 & 0,19 & 1,2 & 12 & 1,3 & 360 & 2,6 \\
\hline 98010 & Myotis mystacinus - Bartfledermaus & Clausthal-Zellerfeld & $\mathrm{m}$ & adult & 10.6.1998 & 4128 & 3 & 5,01 & 3,9 & 3,51 & 4,6 & 0,12 & 0,29 & 7,6 & 0,35 & 170 & 24 \\
\hline 98012 & Pipistrellus pipistrellus - Zwergfledermaus & Osterode a.H. - Ortsteil Freiheit & $\mathrm{m}$ & adult & 2.6.1998 & 4227 & 4 & 3,12 & 3,9 & 3,00 & 130 & 0,55 & 3,0 & 18 & 2,0 & 750 & 15 \\
\hline 98013 & Pipistrellus pipistrellus - Zwergfledermaus & Osterode a.H. - Ortsteil Freiheit & $\mathrm{m}$ & adult & 2.6 .1998 & 4227 & 4 & 3,37 & 3,7 & 3,17 & 66 & 0,62 & 4,6 & 10 & 2,0 & 340 & 56 \\
\hline 98014 & Pipistrellus pipistrellus - Zwergfledermaus & Barbis, Bad Lauterberg i.H. & w & adult & 10.7.1998 & 4328 & 4 & 2,98 & 3,8 & 2,97 & 15 & 0,095 & 7,4 & 13 & 1,4 & 230 & 9,0 \\
\hline 98015 & Myotis mystacinus - Bartfledermaus & Dorste, Stadt Osterode a.H. & w & adult & 14.6.1998 & 4226 & 4 & 3,91 & 3,8 & 3,38 & 5,5 & 0,13 & 0,85 & 7,7 & 0,42 & 160 & \\
\hline 98016 & Pipistrellus pipistrellus - Zwergfledermaus & Osterode a.H. - Ortsteil Freiheit & $\mathrm{m}$ & adult & 4.7.1998 & 4227 & 4 & 3,67 & 3,7 & 3,02 & 140 & 3,7 & 5,4 & 39 & 1,4 & 430 & 93 \\
\hline 98017 & Pipistrellus pipistrellus - Zwergfledermaus & Schwiegershausen, Stadt OHA & $\mathrm{m}$ & adult & 7.6.1998 & 4327 & 1 & 1,48 & 3,4 & 3,16 & 31 & 0,37 & 1,1 & 13 & 1,4 & 850 & 9,7 \\
\hline 98019 & Pipistrellus pipistrellus - Zwergfledermaus & Bad Sachsa & $\mathrm{m}$ & subad. & & 4429 & 1 & 2,78 & 3,9 & 2,77 & 50 & 0,12 & 17 & 14 & 3,2 & 450 & 7,0 \\
\hline 98020 & Pipistrellus pipistrellus - Zwergfledermaus & Osterode a.H. - Ortsteil Freiheit & $\mathrm{m}$ & subad. & 26.6.1998 & 4227 & 4 & 2,95 & 4,4 & 3,18 & 50 & 0,57 & 6,9 & 14 & 8,4 & 1100 & 47 \\
\hline 98022 & Pipistrellus pipistrellus - Zwergfledermaus & Förste, Stadt Osterode a.H. & $\mathrm{m}$ & adult & 20.7.1998 & 4227 & 3 & & & 3,30 & 51 & 0,12 & 11 & 25 & 3,8 & 300 & 9,1 \\
\hline 98023 & Pipistrellus pipistrellus - Zwergfledermaus & Osterode a.H. - Ortsteil Freiheit & $\mathrm{m}$ & adult & 7.6 .1998 & 4227 & 3 & & & 3,27 & 63 & 3,7 & 1,9 & 18 & 2,2 & 300 & 7,8 \\
\hline 98033 & Plecotus auritus - Braunes Langohr & Rhauderfehn, LK Leer & $?$ & adult & 24.09 .1998 & 2710 & 4 & & & & 0,18 & & 1,9 & 6,5 & 1,6 & 130 & 1,8 \\
\hline 98035 & Pipistrellus pipistrellus - Zwergfledermaus & Osterode a.H. & $\mathrm{m}$ & adult & Okt. 1998 & 4227 & 4 & 3,04 & 3,8 & 3,12 & 53 & 1,6 & 2 & 19 & 3,1 & 350 & 129 \\
\hline 98036 & Pipistrellus pipistrellus - Zwergfledermaus & Osterode a.H. & $\mathrm{m}$ & adult & Okt. 1998 & 4227 & 4 & 1,35 & 4,0 & 2,98 & 15 & 1,7 & 6,9 & 44 & 13 & 490 & 29 \\
\hline 98037 & Pipistrellus pipistrellus - Zwergfledermaus & Osterode a.H. & $\mathrm{m}$ & adult & Okt. 1998 & 4227 & 4 & 1,86 & 4,7 & 3,40 & 5,9 & 0,2 & 2,1 & 17 & 2,6 & 220 & 4,7 \\
\hline 98038 & Pipistrellus pipistrellus - Zwergfledermaus & Osterode a.H. & $\mathrm{m}$ & adult & Okt. 1998 & 4227 & 4 & 0,94 & 4,0 & 3,18 & 5,3 & 0,17 & 1,4 & 15 & 2,7 & 230 & 13 \\
\hline 98039 & Pipistrellus pipistrellus - Zwergfledermaus & Osterode a.H. & $\mathrm{m}$ & adult & Okt. 1998 & 4227 & 4 & 1,21 & 3,9 & 3,08 & 7,5 & 0,16 & 1,6 & 17 & 1,6 & 230 & 2,9 \\
\hline 98040 & Pipistrellus pipistrellus - Zwergfledermaus & Osterode a.H. & $\mathrm{m}$ & adult & Okt. 1998 & 4227 & 4 & 1,31 & 4,0 & 3,27 & 33 & 0,65 & 5,8 & 14 & 1,5 & 370 & 35 \\
\hline 98041 & Pipistrellus pipistrellus - Zwergfledermaus & Osterode a.H. & $\mathrm{m}$ & adult & Okt. 1998 & 4227 & 4 & 1,36 & 4,0 & 3,25 & 1,8 & $<0,18$ & 1,1 & 10 & 0,93 & 220 & 17 \\
\hline 98042 & Pipistrellus pipistrellus - Zwergfledermaus & Osterode a.H. & $\mathrm{m}$ & adult & Okt. 1998 & 4227 & 4 & 1,16 & 4,0 & 3,20 & 19 & $<0,18$ & 1,0 & 12 & 0,96 & 210 & 64 \\
\hline 98043 & Pipistrellus pipistrellus - Zwergfledermaus & Osterode a.H. & $\mathrm{m}$ & adult & Okt. 1998 & 4227 & 4 & 3,68 & 4,1 & 3,17 & 7,8 & $<0,2$ & 0,72 & 13 & 0,91 & 230 & 18 \\
\hline 98044 & Pipistrellus pipistrellus - Zwergfledermaus & Osterode a.H. & $\mathrm{m}$ & adult & Okt. 1998 & 4227 & 4 & 0,91 & 4,3 & 3,03 & 4,9 & & 1,5 & 184 & 1,9 & 220 & 11 \\
\hline
\end{tabular}




\begin{tabular}{|c|c|c|c|c|c|c|c|c|c|c|c|c|c|c|c|c|c|}
\hline $\begin{array}{l}\text { Tier } \\
\text { Nr. }\end{array}$ & Art & Fundort & Geschl. & Alter & Funddatum & \begin{tabular}{|c|} 
Blatt \\
Nr. \\
TK 25
\end{tabular} & Quadr. & $\begin{array}{c}\text { Körper } \\
\text { Gew. } \\
\text { [g] }\end{array}$ & $\begin{array}{c}\text { Körper } \\
\text { länge } \\
\text { [cm] }\end{array}$ & $\begin{array}{c}\text { Unter- } \\
\text { arm- } \\
\text { länge } \\
\text { [cm] }\end{array}$ & \begin{tabular}{|c|}
$\mathrm{Pb}$ \\
Haare \\
{$[\mu \mathrm{g} / \mathrm{g}]$}
\end{tabular} & $\begin{array}{c}\text { Cd } \\
\text { Haare } \\
{[\mu \mathrm{g} / \mathrm{g}]}\end{array}$ & $\begin{array}{c}\mathrm{Cr} \\
\text { Haare } \\
{[\mu \mathrm{g} / \mathrm{g}]}\end{array}$ & $\begin{array}{c}\mathrm{Cu} \\
\text { Haare } \\
{[\mu \mathrm{g} / \mathrm{g}]}\end{array}$ & $\begin{array}{c}\mathrm{Ni} \\
\text { Haare } \\
{[\mu \mathrm{g} / \mathrm{g}]}\end{array}$ & $\begin{array}{l}\mathrm{Zn} \\
\text { Haare } \\
{[\mu \mathrm{g} / \mathrm{g}]}\end{array}$ & 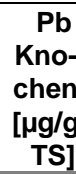 \\
\hline 98045 & Pipistrellus pipistrellus - Zwergfledermaus & Osterode a.H. & $\mathrm{m}$ & adult & Okt. 1998 & 4227 & 4 & 1,11 & 3,8 & 3,19 & 28 & 0,52 & 1,0 & 14 & 2,0 & 260 & 71 \\
\hline 98047 & Pipistrellus pipistrellus - Zwergfledermaus & Osterode a.H. & $\mathrm{m}$ & adult & Okt. 1998 & 4227 & 4 & 0,85 & 4,1 & 3,05 & 15 & 0,08 & 3,5 & 12 & 1,8 & 220 & 2,8 \\
\hline 98052 & Pipistrellus pipistrellus - Zwergfledermaus & Osterode a.H. & w & adult & Okt. 1998 & 4227 & 4 & 1,23 & & 3,10 & & & & & & & 13 \\
\hline 98053 & Pipistrellus pipistrellus - Zwergfledermaus & Osterode a.H. & w & adult & Okt. 1998 & 4227 & 4 & 3,69 & 4,1 & 3,13 & 21 & 0,12 & 2,3 & 12 & 1,3 & 240 & 36 \\
\hline 98054 & Pipistrellus pipistrellus - Zwergfledermaus & Osterode a.H. & w & adult & Okt. 1998 & 4227 & 4 & 1,31 & 4,4 & 3,21 & 6,4 & 0,16 & 2,8 & 11 & 1,2 & 250 & 33 \\
\hline 98055 & Pipistrellus pipistrellus - Zwergfledermaus & Osterode a.H. & w & adult & Okt. 1998 & 4227 & 4 & 4,02 & 4,1 & 3,16 & & & & & & & 17 \\
\hline 98056 & Pipistrellus pipistrellus - Zwergfledermaus & Osterode a.H. & w & adult & Okt. 1998 & 4227 & 4 & 3,83 & 4,1 & 3,30 & 3,5 & 0,067 & 0,68 & 10 & 0,74 & 210 & 2,6 \\
\hline 98057 & Pipistrellus pipistrellus - Zwergfledermaus & Osterode a.H. & w & adult & Okt. 1998 & 4227 & 4 & 1,91 & 4,0 & 3,01 & & & & & & & 15 \\
\hline 98058 & Pipistrellus pipistrellus - Zwergfledermaus & Osterode a.H. & w & adult & Okt. 1998 & 4227 & 4 & 1,28 & 4,2 & 3,30 & 160 & 0,10 & 2,0 & 12 & 2,9 & 220 & 94 \\
\hline 98059 & Pipistrellus pipistrellus - Zwergfledermaus & Osterode a.H. & w & adult & Okt. 1998 & 4227 & 4 & 3,07 & 4,0 & 3,30 & & & & & & & 66 \\
\hline 98060 & Pipistrellus pipistrellus - Zwergfledermaus & Osterode a.H. & w & adult & Okt. 1998 & 4227 & 4 & 3,66 & 4,2 & 3,09 & 150 & 0,42 & 26 & 11 & 1,3 & 510 & 33 \\
\hline 98066 & Pipistrellus pipistrellus - Zwergfledermaus & Osterode a.H. & $\mathrm{m}$ & adult & Okt. 1998 & 4227 & 4 & 1,18 & 4,0 & 2,95 & 19 & 0,14 & 2,3 & 40 & 1,3 & 230 & 7,5 \\
\hline 98067 & Pipistrellus pipistrellus - Zwergfledermaus & Osterode a.H. & $\mathrm{m}$ & adult & Okt. 1998 & 4227 & 4 & 1,01 & 4,2 & 3,17 & 52 & 0,13 & 1,8 & 10 & 1,5 & 230 & 85 \\
\hline 98068 & Pipistrellus pipistrellus - Zwergfledermaus & Osterode a.H. & $\mathrm{m}$ & adult & Okt. 1998 & 4227 & 4 & 1,00 & 4,3 & 3,14 & & & & & & & 14 \\
\hline 98069 & Pipistrellus pipistrellus - Zwergfledermaus & Osterode a.H. & $\mathrm{m}$ & adult & Okt. 1998 & 4227 & 4 & 3,19 & 4,0 & 2,87 & 7,8 & 0,18 & 0,83 & 29 & 0,79 & 220 & 8,1 \\
\hline 98070 & Pipistrellus pipistrellus - Zwergfledermaus & Osterode a.H. & $\mathrm{m}$ & adult & Okt. 1998 & 4227 & 4 & 0,92 & & 3,15 & & & & & & & 11 \\
\hline 98071 & Pipistrellus pipistrellus - Zwergfledermaus & Osterode a.H. & $\mathrm{m}$ & adult & Okt. 1998 & 4227 & 4 & 0,87 & 4,1 & 3,03 & & & & & & & 17 \\
\hline 98072 & Pipistrellus pipistrellus - Zwergfledermaus & Osterode a.H. & $\mathrm{m}$ & adult & Okt. 1998 & 4227 & 4 & 3,64 & 3,9 & 3,15 & 11 & 0,15 & 1,6 & 24 & 1,6 & 240 & 20 \\
\hline 98073 & Pipistrellus pipistrellus - Zwergfledermaus & Osterode a.H. & $\mathrm{m}$ & adult & Okt. 1998 & 4227 & 4 & 1,06 & 4,1 & 3,14 & & & & & & & 25 \\
\hline 98074 & Pipistrellus pipistrellus - Zwergfledermaus & Osterode a.H. & $\mathrm{m}$ & adult & Okt. 1998 & 4227 & 4 & 1,31 & 4,1 & 3,24 & & & & & & & 56 \\
\hline 98075 & Pipistrellus pipistrellus - Zwergfledermaus & Osterode a.H. & $\mathrm{m}$ & adult & Okt. 1998 & 4227 & 4 & 1,41 & 4,4 & 3,05 & & & & & & & 12 \\
\hline 98088 & Pipistrellus pipistrellus - Zwergfledermaus & Salzgitter-Bruchmachtersen & w & adult & 20.12.1998 & 3827 & 2 & 4,04 & 4,0 & 3,20 & 25 & 0,25 & 2,1 & 25 & 1,4 & 260 & 31 \\
\hline 98089 & Pipistrellus pipistrellus - Zwergfledermaus & Osterode-Förste & w & adult & 14.9.1998 & 4227 & 3 & 1,68 & 3,9 & 3,25 & & & & & & & 8,2 \\
\hline 98092 & Pipistrellus pipistrellus - Zwergfledermaus & Bremen, Lilienthal & w & adult & 15.11.1998 & 2819 & 2 & 3,23 & 4,4 & 3,25 & 18 & 0,17 & 3,1 & 47 & 3,6 & 400 & 6,7 \\
\hline 98093 & Pipistrellus pipistrellus - Zwergfledermaus & Grußendorf & $\mathrm{m}$ & adult & 6.98 & 3430 & 3 & 2,62 & 3,3 & 3,11 & 77 & 0,51 & 3,1 & 44 & 3,2 & 350 & 13 \\
\hline 99001 & Myotis myotis - Gr. Mausohr & Northeim, Stadtgebiet (Heimat- & w & adult & 3/1999 & 4225 & 4 & 8,70 & 6,3 & 5,84 & & & & & & & 5,6 \\
\hline 99002 & Myotis myotis - Gr. Mausohr & Northeim, Stadtgebiet (Heimat- & w & adult & 3/1999 & 4225 & 4 & 7,65 & 6,3 & 5,89 & & & & & & & 5,1 \\
\hline 99003 & Myotis myotis - Gr. Mausohr & Northeim, Stadtgebiet (Heimat- & w & adult & $3 / 1999$ & 4225 & 4 & 3,31 & 7,0 & 5,40 & & & & & & & 4,6 \\
\hline 99004 & Myotis myotis - Gr. Mausohr & Northeim, Stadtgebiet (Heimat- & w & adult & 3/1999 & 4225 & 4 & 7,84 & 7,3 & 5,74 & & & & & & & 5,3 \\
\hline 99005 & Myotis myotis - Gr. Mausohr & Northeim, Stadtgebiet (Heimat- & w & adult & $3 / 1999$ & 4225 & 4 & 1,87 & 5,9 & 4,79 & & & & & & & 4,8 \\
\hline 99027 & Pipistrellus pipistrellus - Zwergfledermaus & Bückeburg & $?$ & adult & Juli 1999 & 3720 & 1 & 0,95 & 3,6 & 3,12 & 4,5 & 0,27 & 0,81 & 20 & 1,2 & 220 & 20 \\
\hline
\end{tabular}


Anhang 6: Zuordnung der Haarbleigehalte von Zwergfledermäusen (Pipistrellus pipistrellus) aus dem Betrachtungszeitraum 1996-1999 zum Fundort

\begin{tabular}{|c|c|c|c|c|c|c|c|c|}
\hline Tier-Nr & Fundort & Lebensraumcharakterisierung & Geschlecht & Alter & Funddatum & TK25 & Quadrant & Haarblei \\
\hline 96016 & Kreiensen & $\begin{array}{l}\text { In unmittelbarer Nähe der stark be- } \\
\text { fahrenen Bundesstraße B } 64\end{array}$ & $\mathrm{~m}$ & subad. & 18.9 .96 & 4125 & 2 & 140 \\
\hline 96021 & Greene Landkreis NOM & $\begin{array}{l}\text { Quartier in einer Brücke der stark } \\
\text { befahrenen Bundesstraße B } 64\end{array}$ & w & adult & 12.6.96 & 4125 & 2 & 120 \\
\hline 96027 & Hannover, Stadtgebiet & Großstadt & w & subad. & 9.8 .96 & 3624 & 1 & $(93)^{*}$ \\
\hline 98093 & Grußendorf, LK Gifhorn & ländliche Region & $\mathrm{m}$ & adult & 6.98 & 3430 & 3 & 77 \\
\hline 96001 & Hannover-Kleefeld & Großstadt & $\mathrm{m}$ & adult & 11.8 .96 & 3624 & 2 & $(64)^{\star}$ \\
\hline 96003 & Hannover, Rühmkorfstraße & Großstadt & $\mathrm{m}$ & adult & 22.8.1996 & 3524 & 3 & 63 \\
\hline 96002 & Hannover, Rühmkorfstr. & Großstadt & $\mathrm{m}$ & adult & 22.8.1996 & 3524 & 3 & 38 \\
\hline 96026 & Hannover, Stadtgebiet & Großstadt & $\mathrm{m}$ & subad. & 24.8 .96 & 3624 & 1 & 26 \\
\hline 98088 & Salzgitter-Bruchmachtersen & Mittelzentrum & w & adult & 20.12.1998 & 3827 & 2 & 25 \\
\hline 96029 & Hannover, Stadtgebiet & Großstadt & $\mathrm{m}$ & adult & 23.8 .96 & 3624 & 1 & 23 \\
\hline 96028 & Bothel, LK Rothenburg/Wümme & $\begin{array}{l}\text { ländliche Region, zwischen zwei } \\
\text { Bundesstraßen gelegen }\end{array}$ & - & subad. & - & 2923 & 1 & 22 \\
\hline 98092 & Bremen, Lilienthal & Großstadtrandlage, ländlich geprägt & w & adult & 15.11 .1998 & 2819 & 2 & 18 \\
\hline 96023 & Rhumspringe Landkreis Göttingen & ländliche Region & w & subad. & 23.7.96 & 4427 & 2 & 14 \\
\hline 97013 & Elvershausen Landkreis NOM & ländliche Region & $\mathrm{m}$ & subad. & 2.8 .97 & 4226 & 4 & 8,6 \\
\hline 97012 & Marke Landkreis Osterode & ländliche Region & $\mathrm{m}$ & adult & 1.8 .97 & 4226 & 4 & 8 \\
\hline 99027 & Bückeburg, LK Schaumburg & Mittelzentrum in ländlicher Region & $?$ & adult & Juli 1999 & 3720 & 1 & 4,5 \\
\hline 97014 & Harriehausen Landkreis NOM & ländliche Region & $\mathrm{m}$ & subad. & 6.7 .97 & 4126 & 4 & 3,4 \\
\hline
\end{tabular}

*) In Klammern (..): nicht gewertete Datensätze aufgrund extremer Zinkwerte (Zinkausreißer) 
Anhang 7: Analysenergebnisse der Kotuntersuchungen unterschiedlicher Fledermausarten. Alle Meßwerte in mg/kg TS

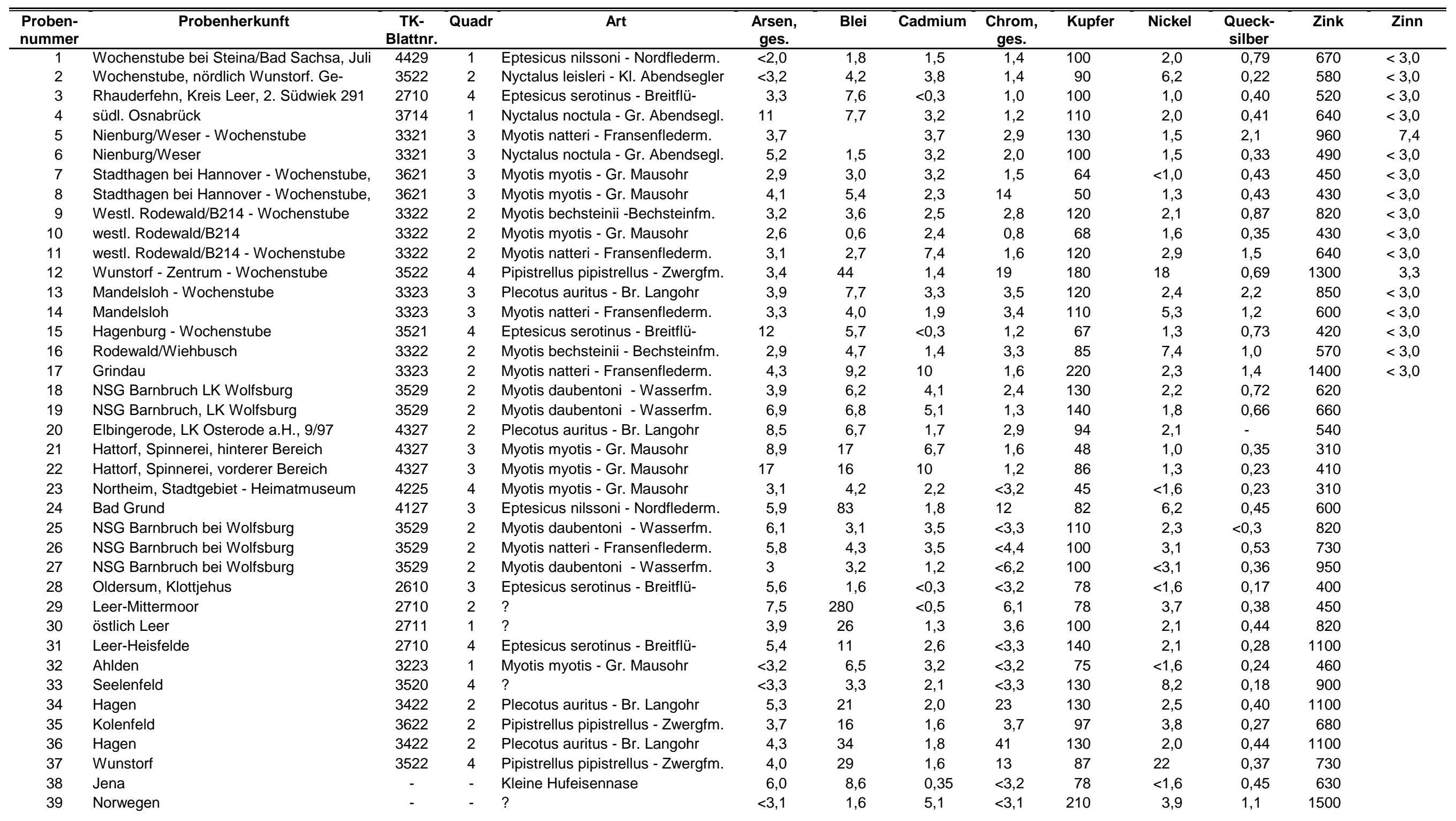




\begin{tabular}{|c|c|c|c|c|c|c|c|c|c|c|c|c|c|}
\hline $\begin{array}{l}\text { Proben- } \\
\text { nummer }\end{array}$ & Probenherkunft & $\begin{array}{c}\text { TK- } \\
\text { Blattnr. }\end{array}$ & Quadr & Art & $\begin{array}{l}\text { Arsen, } \\
\text { ges. }\end{array}$ & Blei & Cadmium & $\begin{array}{c}\text { Chrom, } \\
\text { ges. }\end{array}$ & Kupfer & Nickel & $\begin{array}{l}\text { Queck- } \\
\text { silber }\end{array}$ & Zink & $\overline{Z \text { Zinn }}$ \\
\hline 40 & Moringen, Kirche & 4225 & 3 & ? & $<4,3$ & 120 & 2,2 & 4,3 & 89 & 3,6 & 0,39 & 460 & \\
\hline 41 & Steinbergen & 3420 & 4 & Myotis myotis - Gr. Mausohr & $<2,8$ & 6,2 & 2,0 & $<2,8$ & 63 & 1,4 & 0,28 & 430 & \\
\hline 43 & Seelenfeld & 3520 & 4 & $?$ & $<3,1$ & 4,3 & 2,4 & $<3,1$ & 58 & $<1,6$ & 0,16 & 440 & \\
\hline 45 & Seelenfeld & 3520 & 4 & ? & $<3,3$ & 2,0 & 2,3 & $<3,3$ & 110 & 1,7 & 0,23 & 1400 & \\
\hline 46 & Seelenfeld & 3520 & 4 & ? & $<4,2$ & 3,1 & 1,9 & $<4,2$ & 120 & $<2,1$ & 0,27 & 1300 & \\
\hline 56 & Zorge & 4329 & 4 & Pipistrellus pipistrellus - Zwergfm. & 12 & 4300 & 18 & 34 & 4600 & 6,6 & 15 & 14000 & \\
\hline 57 & Bad Gandersheim & 4126 & 1 & Pipistrellus pipistrellus - Zwergfm. & 0,56 & 4,9 & 2,0 & 20 & 76 & $<4,5$ & 0,68 & 500 & \\
\hline
\end{tabular}




\section{Danksagung}

Die vorliegenden Untersuchungen wurden möglich durch die langjährige aktive Arbeit der ehrenamtlichen Fledermausschutzbeauftragten in Niedersachsen und vieler Fledermausfreunde, die Fledermaustotfunde oder vergebliche Aufzuchtversuche von erkrankten Tieren dem NLÖ zur Konservierung übergaben oder Kotproben aus den ihnen bekannten Quartieren sammelten und dem Verfasser zur weiteren Bearbeitung übersandten. In diesem Zusammenhang danke ich Frau A. TILSNER, Rhauderfehn, und Herrn D. SCHLEGEL, Wunstorf. Besonderen Dank möchte ich Herrn W. RACKOW, Osterode am Harz, für die anregenden Diskussionen und seinen unermüdlichen Einsatz für den Fledermausschutz im südlichen Niedersachsen sagen. Herrn Dr. LÖHMER, Tiermedizinische Hochschule Hannover, danke ich für die Unterstützung bei der Probensichtung. Für die Durchführung der Platinanalysen danke ich Herrn Dr. ALT, Institut für Spektrochemie, Dortmund.

Besonderer Dank gilt auch den Betreuern dieser Arbeit. Für die Übernahme der Betreuung dieser Arbeit seitens der Biologischen Fakultät danke ich Herrn Prof. Dr. M. SCHAEFER, Zoologisches Institut der Universität Göttingen. Herrn PD Dr. Dr. H. KIJEWSKI, Institut für Rechtsmedizin der Universität Göttingen, danke ich für die Unterstützung bei der Methodenentwicklung zur Haaranalytik und den zahlreichen anregenden fachlichen Gesprächen. Herrn PD

Dr. H. SCHUTKOWSKI, University of Bradford, danke ich für die Bereitschaft zur Übernahme des Korreferates.

Vor allem danke ich auch meiner Ehefrau Brigitta Stammer. Sie organisierte über einen Zeitraum von zwei Jahren fast allein unseren gemeinsamen beruflichen und privaten Alltag und war kritische Leserin und Lektorin des Textes. 


\section{Lebenslauf Rainer Hartmann}

Geburtsdatum: $\quad$ 25.01.1957 in Hamburg als Sohn des Chemiefachwerkers Albert Hartmann und dessen Ehefrau Helga Hartmann

Staatsangehörigkeit: deutsch

Personenstand: verheiratet

\section{Schulische Ausbildung und Studium:}

13.06.1977 Erlangung der Allg. Hochschulreife am Lohmühlengymnasium in Hamburg

02.11.1978 Beginn des Biologiestudiums an der Georg August Universität Göttingen nach Absolvierung des Grundwehrdienstes in Goslar

16.07.1980 Absolvierung der Diplomvorprüfung in der Fachrichtung Biologie

16.05.1983 Abschluß des Biologiestudiums mit bestandener Diplomprüfung. Titel der Diplomarbeit: „Untersuchungen über Hydrologie und aquatische Fauna in einem Bergwerk des Harzes, unter besonderer Berücksichtigung des Höhlenflohkrebses Niphargus schellenbergi". Die Arbeit wurde unter Betreuung des Max-Planck-Institutes für Limnologie, Außenstelle Schlitz, sowie des II. Zool. Instituts der Universität Göttingen, Abt. Ökologie, erstellt

14.02.1984 Absolvierung der Diplomvorprüfung in der Fachrichtung Geologie / Paläontologie

\section{Beruflicher Werdegang:}

1983-1986 Mitarbeiter im Sonderforschungsbereich "terrestrische Ökosysteme" der Deutschen Forschungsgemeinschaft am II. Zoologischen Institut der Universität Göttingen

01.01.1983 Gründung der Firma RAINER HARTMANN, Büro für angewandte Biologie und Geologie in Göttingen

seit

01.01.1993 Geschäftsführender Gesellschafter der RAINER HARTMANN GmbH.

01.02.1993 Ernennung zum öffentlich bestellten und vereidigten Sachverständigen für Unfälle mit wassergefährdenden Stoffen durch die IHK Hannover-Hildesheim 


\section{Sonstige berufliche Tätigkeiten:}

seit SS 1992 Lehrbeauftragter für Umweltmeßtechnik an der Fachhochschule Hildesheim-Holzminden-Göttingen, FB Forstwirtschaft und Umweltmanagement.

seit

01.06.1993 Vorstandsmitglied im „Verband deutscher Untersuchungslaboratorien e.V.“ (VDU), Sitz Hannover.

seit

09.07.1997 Vorstandsvorsitzender vom „Verband deutscher Untersuchungslaboratorien e.V." (VDU), Sitz Hannover.

06/97-10/2000 Mitglied in der ad-hoc AG „Akkreditierung von Meßstellen und Prüflabors“ des AK 4 der Länderarbeitsgemeinschaft Boden bis zu dessen Auflösung nach Einführung und Umsetzung des Bundesbodenschutzgesetzes sowie Verabschiedung der Richtlinien zur Notifizierung auf der 18. Sitzung der LABO am 11./12.2000 in Potsdam.

seit 06/2000 Mitglied im Arbeitskreis Bodenschutz der 4. Regierungskommission des Landes Niedersachsen.

Göttingen, 13. Dezember 2000 